\title{
Graph colorings \\ and digraph subdivisions
}

Phablo Fernando Soares Moura

THESIS SUBMITTED TO

THE

Instituto DE MATEMÁTiCA E EstatísticA

AT

Universidade DE SÃo PaUlo

FOR THE DEGREE

$\mathrm{OF}$

DOCTOR OF SCIENCE

Doctoral program in Computer Science

Advisor: Dr. rer. nat. Yoshiko Wakabayashi

We gratefully acknowledge the support of

São Paulo Research Foundation (FAPESP)

under grant numbers 2013/19179-0, 2015/11930-4

São Paulo, May 18, 2017 



\title{
Colorações de grafos $\mathrm{e}$ subdivisões de digrafos
}

\author{
Phablo Fernando Soares Moura
}

TESE APRESENTADA

$\mathrm{AO}$

Instituto DE MATEMÁticA E EstatísticA

DA

Universidade DE SÃo PaUlo

PARA

OBTENÇÃO DO TÍTULO

$\mathrm{DE}$

DOUTOR EM CIÊNCIAS

Programa de pós-graduação em Ciência da Computação Orientadora: Dr. rer. nat. Yoshiko Wakabayashi

Agradecemos o apoio da Fundação de Amparo à Pesquisa do Estado de São Paulo (FAPESP) processos 2013/19179-0, 2015/11930-4 


\section{Colorações de grafos e subdivisões de digrafos}

Esta versão da tese contém as correções e alterações sugeridas pela Comissão Julgadora durante a defesa da versão original do trabalho, realizada em 30/03/2017. Uma cópia da versão original está disponível no Instituto de Matemática e Estatística da Universidade de São Paulo.

Comissão Julgadora:

- Prof ${ }^{\mathrm{a}}$. Dr ${ }^{\mathrm{a}}$. Yoshiko Wakabayashi (orientadora) - IME-USP

- Prof ${ }^{\mathrm{a}}$. Dr ${ }^{\mathrm{a}}$. Celina Miraglia Herrera de Figueiredo - UFRJ

- Prof. Dr. Cid Carvalho de Souza - UNICAMP

- Prof. Dr. Manoel Bezerra Campêlo Neto - UFC

- Prof. Dr. Guilherme Oliveira Mota - IME-USP 


\section{Agradecimentos}

Primeiramente, agradeço à minha orientadora Yoshiko, profissional extremamente competente e pessoa incrível, por tantos anos de apoio e dedicação a este trabalho. Nossas conversas e seus conselhos me ensinaram muito além do que está escrito em livros. Sinto-me muitíssimo privilegiado por ser seu aluno.

Agradeço aos meus pais, que me mostraram a importância da educação desde muito cedo e me apoiaram de todas as formas ao longo desses anos. Um agradecimento muito especial também às minhas irmãs, Flávia e Fabiana. Obrigado ao Bruno por todo o apoio e torcida pelo meu sucesso. Jamais teria conseguido chegar até este momento sem o apoio e o carinho da minha família.

Agradeço à Eva, moje krásná holka, pelas muitas palavras de incentivo e por todo carinho que me fizeram ter tranquilidade e sorrir mesmo nos dias mais difíceis. Meus dias ao seu lado são sempre mais felizes.

Por fim, agradeço a Fundação de Amparo à Pesquisa do Estado de São Paulo (FAPESP) pelo apoio financeiro dedicado ao desenvolvimento desta tese por meio dos processos 2013/19179-0 e 2015/11930-4. 


\section{Resumo}

MOURA, P. F. S. Colorações de grafos e subdivisões de digrafos. 2017. 131 f. Tese de doutorado - Instituto de Matemática e Estatística, Universidade de São Paulo, São Paulo, 2017.

O problema de coloração de grafos é um problema clássico em teoria dos grafos cujo objetivo é particionar o conjunto de vértices em um número mínimo de conjuntos estáveis. Nesta tese apresentamos nossas contribuições sobre três problemas de coloração de grafos e um problema relacionado a uma antiga conjectura sobre subdivisão de digrafos. Primeiramente, abordamos o problema de recoloração convexa no qual é dado um grafo arbitrariamente colorido $G$ e deseja-se encontrar uma recoloração de peso mínimo tal que cada classe de cor induza um subgrafo conexo de $G$. Mostramos resultados sobre inaproximabilidade, introduzimos uma formulação linear inteira que modela esse problema, e apresentamos alguns resultados computacionais usando uma abordagem de geração de colunas. O problema de $k$-upla coloração é uma generalização do problema clássico de coloração de vértices e consiste em cobrir o conjunto de vértices de um grafo com uma quantidade mínima de conjuntos estáveis de tal forma que cada vértice seja coberto por pelo menos $k$ conjuntos estáveis (possivelmente idênticos). Apresentamos uma formulação linear inteira para esse problema e fazemos um estudo detalhado do politopo associado a essa formulação. $\mathrm{O}$ último problema de coloração estudado nesta tese é o problema de orientação própria. Ele consiste em orientar o conjunto de arestas de um dado grafo de tal forma que vértices adjacentes possuam graus de entrada distintos e o maior grau de entrada seja minimizado. Claramente, os graus de entrada induzem uma partição do conjunto de vértices em conjuntos estáveis, ou seja, induzem uma coloração (no sentido convencional) dos vértices. Nossas contribuições nesse problema são em complexidade computacional e limitantes superiores para grafos bipartidos. Finalmente, estudamos um problema relacionado a uma conjectura de Mader, dos anos oitenta, sobre subdivisão de digrafos. Esta conjectura afirma que, para cada digrafo acíclico $H$, existe um inteiro $f(H)$ tal que todo digrafo com grau mínimo de saída pelo menos $f(H)$ contém uma subdivisão de $H$ como subdigrafo. Damos evidências para essa conjectura mostrando que ela é válida para classes particulares de digrafos acíclicos.

Palavras-chave: coloração de grafos, recoloração convexa, orientação de grafos, subdivisão de digrafos, poliedro, geração de colunas, inaproximabilidade, complexidade computacional. 


\section{Abstract}

MOURA, P. F. S. Graph colorings and digraph subdivisions. 2017. 131 pp. Doctoral thesis - Instituto de Matemática e Estatística, Universidade de São Paulo, São Paulo, 2017.

The vertex coloring problem is a classic problem in graph theory that asks for a partition of the vertex set into a minimum number of stable sets. This thesis presents our studies on three vertex (re)coloring problems on graphs and on a problem related to a long-standing conjecture on subdivision of digraphs. Firstly, we address the convex recoloring problem in which an arbitrarily colored graph $G$ is given and one wishes to find a minimum weight recoloring such that each color class induces a connected subgraph of $G$. We show inapproximability results, introduce an integer linear programming (ILP) formulation that models the problem and present some computational experiments using a column generation approach. The $k$-fold coloring problem is a generalization of the classic vertex coloring problem and consists in covering the vertex set of a graph by a minimum number of stable sets in such a way that every vertex is covered by at least $k$ (possibly identical) stable sets. We present an ILP formulation for this problem and show a detailed polyhedral study of the polytope associated with this formulation. The last coloring problem studied in this thesis is the proper orientation problem. It consists in orienting the edge set of a given graph so that adjacent vertices have different in-degrees and the maximum in-degree is minimized. Clearly, the in-degrees induce a partition of the vertex set into stable sets, that is, a coloring (in the conventional sense) of the vertices. Our contributions in this problem are on hardness and upper bounds for bipartite graphs. Finally, we study a problem related to a conjecture of Mader from the eighties on subdivision of digraphs. This conjecture states that, for every acyclic digraph $H$, there exists an integer $f(H)$ such that every digraph with minimum out-degree at least $f(H)$ contains a subdivision of $H$ as a subdigraph. We show evidences for this conjecture by proving that it holds for some particular classes of acyclic digraphs.

Keywords: graph coloring, convex recoloring, graph orientation, digraph subdivision, polyhedral study, column generation, inapproximability, computational complexity. 


\section{Contents}

List of Figures $\quad$ xi

I Preliminaries 1

1 Introduction 3

2 Basic concepts, terminology and notation $\quad 7$

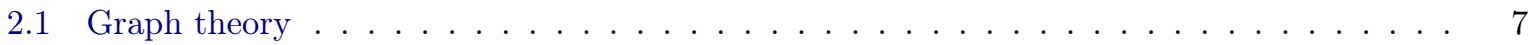

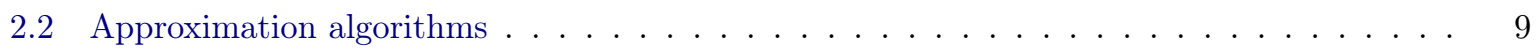

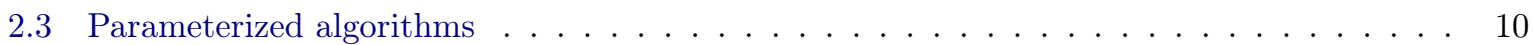

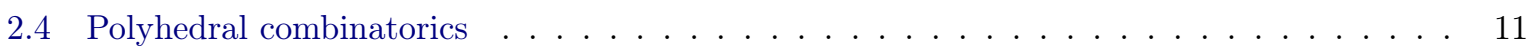

$\begin{array}{ll}\text { II Convex recoloring } & 13\end{array}$

3 The convex recoloring problem $\quad 15$

3.1 Basic definitions . . . . . . . . . . . . . . . . . . . . . . 15

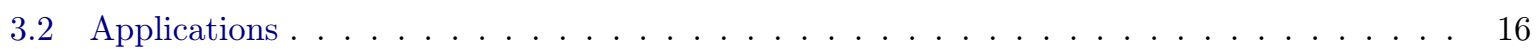

3.3 Known results . . . . . . . . . . . . . . . . . . . . . . . . . 16

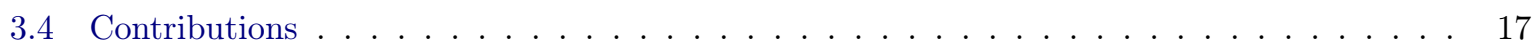

4 Hardness results $\quad 19$

4.1 Approximation thresholds . . . . . . . . . . . . . . . . . . . . . . . . . . . . 19

$4.2 \mathcal{W}[2]$-hardness of a parameterized unweighted version $\ldots \ldots \ldots \ldots \ldots \ldots \ldots$

5 Column generation approach $\quad 27$

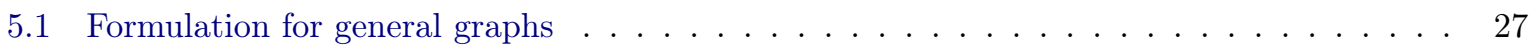

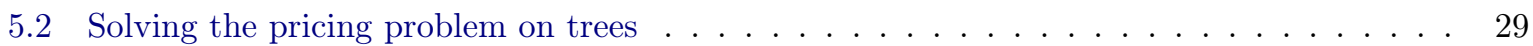

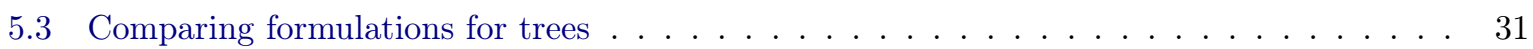

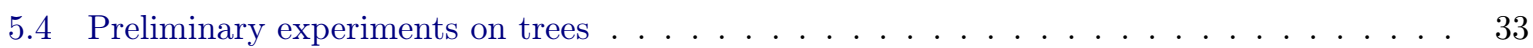

$\begin{array}{lll}\text { III On } k \text {-fold coloring } & 37\end{array}$

$6 \quad$ The $k$-fold coloring problem $\quad 39$

6.1 Basic definitions and known results . . . . . . . . . . . . . . . . . . . . 39

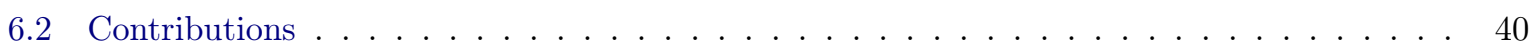


7 Representatives polytope $\quad 43$

7.1 Formulation . . . . . . . . . . . . . . . . . . . . . . . 43

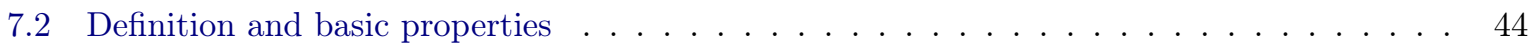

$\begin{array}{llr}8 & \text { Inherited facets } & 47\end{array}$

8.1 Facets from $\mathcal{P}_{k}(G)$ to $\mathcal{P}_{k+\ell}(G) \ldots \ldots \ldots \ldots \ldots \ldots \ldots$. . . . . . . . . . . . . . . . . . . . .

8.2 Facets from $\mathcal{P}_{1}\left(G \circ K_{k}\right)$ to $\mathcal{P}_{k}(G) \ldots \ldots \ldots \ldots \ldots \ldots \ldots \ldots$

8.3 Facets from stable set polytopes to $\mathcal{P}_{k}(G) \ldots \ldots \ldots \ldots \ldots \ldots$

$9 \begin{array}{lr}\text { Facets induced by critical subgraphs } & \mathbf{5 7}\end{array}$

9.1 Critical graphs . . . . . . . . . . . . . . . . . . . . . . . . 57

9.2 Web facets. . . . . . . . . . . . . . . . . . . . . . 59

9.3 Antiweb facets . . . . . . . . . . . . . . . . . . . . . 62

IV Proper orientation $\quad 69$

10 The proper orientation problem $\quad \mathbf{7 1}$

10.1 Basic definitions . . . . . . . . . . . . . . . . . . . . . . 71

10.2 Known results . . . . . . . . . . . . . . . . . . . . . . . . . . 72

10.3 Contributions . . . . . . . . . . . . . . . . . . . . . . . 72

11 Upper bounds for bipartite graphs $\quad 75$

11.1 General bipartite . . . . . . . . . . . . . . . . . . . . . . . . . 75

11.2 Trees . . . . . . . . . . . . . . . . . . . . . . . . 80

12 Hardness results $\quad 85$

12.1 Planar subcubic graphs . . . . . . . . . . . . . . . . . . . . . 85

12.2 Planar bipartite graphs . . . . . . . . . . . . . . . . . . . . . . . . 88

$\begin{array}{llr}\text { V Digraph subdivision } & 93\end{array}$

13 Finding subdivisions of digraphs $\quad 95$

13.1 Basic definitions and known results . . . . . . . . . . . . . . . . . . . . . . 95

13.2 Contributions . . . . . . . . . . . . . . . . . . . . . . 98

14 Digraphs with large out-degree $\quad 99$

14.1 Subdivisions of oriented paths . . . . . . . . . . . . . . . . . . . . . . . 99

14.2 Subdivisions of in-arborescences . . . . . . . . . . . . . . . . . . . . . . 100

$\begin{array}{lll}\text { VI Conclusion } & 103\end{array}$

15 Concluding remarks and open questions $\quad 105$

15.1 Convex recoloring . . . . . . . . . . . . . . . . . . . . . . . 105

15.2 On $k$-fold coloring . . . . . . . . . . . . . . . . . . . . . . . . . 106

15.3 Proper orientation . . . . . . . . . . . . . . . . . . . . . . 107

15.4 Subdivisions of digraphs . . . . . . . . . . . . . . . . . . . . . . 108 


\section{List of Figures}

4.1 Gadgets used in the proof of Theorem 4.2. . . . . . . . . . . . . . . . . . . . . 20

4.2 Gadgets used in the proof of Theorem 4.3. . . . . . . . . . . . . . . . . . 21

4.3 Gadgets used in the proof of Theorem 4.7. . . . . . . . . . . . . . . . . . . . . . 25

4.4 Graph obtained from the formula $\left(x_{1} \vee \neg x_{2} \vee x_{3}\right)$. . . . . . . . . . . . . . . 25

6.1 Examples of $k$-fold $x$-colorings of a cycle $C_{5} \ldots \ldots \ldots \ldots \ldots$

8.1 Example of polytope $P$, and two affine functions $\Lambda$ and $\Pi \ldots \ldots \ldots \ldots$. . . . . . . 52

9.1 Example of a web and an antiweb. . . . . . . . . . . . . . . . . . 5 57

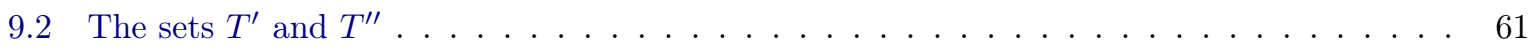

9.3 Illustration of $v_{1}, v_{2}, N_{1}$ and $N_{2} \ldots \ldots \ldots \ldots \ldots \ldots \ldots$

11.1 A bipartite graph $B_{3}$ for which $\vec{\chi}\left(B_{3}\right)=\Delta\left(B_{3}\right)=3 \ldots \ldots \ldots \ldots \ldots$. . . . . . . 79

11.2 Rooted trees $R_{2}$ and $R_{3}$. . . . . . . . . . . . . . . . . . . . . . . . 80

12.1 The variable gadget $\mathrm{VG}_{i}$ and the clause gadget $\mathrm{CG}_{j} \ldots \ldots \ldots \ldots$. . . . . . . 86

12.2 Suitable clause orientations. . . . . . . . . . . . . . . . . . . . . . . 88

12.3 The bipartite graph $B_{5} \ldots \ldots \ldots \ldots$. . . . . . . . . . . . . . . . . . . . . 88

12.4 Every dashed circle represents a copy of $B_{5} \ldots \ldots \ldots \ldots \ldots$. . . . . . . . . . 90

12.5 Two proper 3 -orientations of $B_{5} \ldots \ldots \ldots \ldots \ldots \ldots$. . . . . . . . . . . . . . 91

12.6 Suitable orientations of the gadget $x_{i} \ldots \ldots \ldots \ldots \ldots \ldots$. . . . . . . . . . . . 91

12.7 Suitable 3 -orientations of the clause gadgets. . . . . . . . . . . . . . . . . . . . 92

13.1 Example of subdivision of a digraph. . . . . . . . . . . . . . . . . . . . 95

13.2 Example of a complete digraph and a transitive tournament. . . . . . . . . . . . . 96

14.1 Example of oriented path. . . . . . . . . . . . . . . . . . . . . . 99

14.2 Examples of in-arborescences. . . . . . . . . . . . . . . . . . 101 
xii LIST OF FIGURES 


\section{Part I}

\section{Preliminaries}





\section{Chapter 1}

\section{Introduction}

Many combinatorial problems can be viewed as covering, packing, or partition problems. Given a set $S$ and a set $\Sigma$ of subsets of $S$, in a covering problem we seek a set $\mathcal{T} \subseteq \Sigma$ such that, for each $s$ in $S$, there exists $T$ in $\mathcal{T}$ such that $s \in T$. In a packing problem, we search for $\mathcal{T} \subseteq \Sigma$ such that, for each element $s$ in $S$, there exists at most one set $T$ in $\mathcal{T}$ with $s \in T$. We are interested in coverings of minimum cardinality for the former problem, and in packings of maximum cardinality for the latter. In a partition problem, we aim to find a covering that is also a packing, that is, we seek $\mathcal{T} \subseteq \Sigma$ such that the elements belonging to $\mathcal{T}$ are pairwise disjoint and $\bigcup_{T \in \mathcal{T}} T=S$. One may be interested in partitions either of maximum or minimum cardinality. The objects of study may be graphs, sequences, or other discrete structure. In this thesis, we restrict ourselves to problems on graphs.

Among the classic covering problems, we mention the minimum edge cover and the minimum vertex cover. For the first problem, given a graph $G$, we have $S=V(G)$ and $\Sigma=E(G)$. In this case, we are interested in finding a set $\mathcal{T}$ of edges of $G$ such that every vertex of the graph is endpoint of some edge in $\mathcal{T}$. For the second problem, we have $S=E(G)$ and $\Sigma=\{\delta(v): v \in V(G)\}$, where $\delta(v)$ denotes the set of edges in $G$ that are incident to $v$. In this case, we search for a family $\mathcal{T}$ of sets of edges of $G$ such that, for every edge $e$ in the graph, there exists $\delta(v) \in \mathcal{T}$ with $e \in \delta(v)$, or equivalently, there exists a vertex $v$ such that $e$ is incident to $v$. We observe that the first problem is easier than the second: it is known that one can find a minimum edge cover in polynomial time, but the minimum vertex cover problem is $\mathcal{N} \mathcal{P}$-hard. As examples of packing problems, we mention packing of paths (between two fixed vertices), circuits, and cliques.

As examples of partition problems, we present two of the most studied problems in graph theory: edge coloring and vertex coloring. In the first problem, we partition the edge set of a graph into a minimum number of matchings. In the vertex coloring problem, we aim to partition the set of vertices of a graph into a minimum number of stable sets (color classes). From an algorithmic point of view, both problems are hard. However, there exists a vast literature containing structural results on these problems.

Instead of asking for a partition, a natural variant of the vertex coloring problem is to search for a minimum covering by stable sets that covers every vertex a given (fixed) number of times. One may also look for a minimum partition of the vertex set into stable sets with particular properties, e.g. stable sets induced by "proper" orientations of the graph. Alternatively, instead of stable sets, one may search for a partition of the vertex set into sets that induce connected subgraphs. In this case, each possible partition is assigned a weight and the aim is to find a partition of minimum total weight rather than minimizing the size of the partition. This thesis presents our studies on three coloring problems on graphs and on a problem related to a long-standing conjecture about subdivision of digraphs. 
Firstly, we focus on the coloring problems. We study a problem in which we aim to find a minimum weight partition of vertex set with the property that each element of this partition induces a connected subgraph of the input graph. This is the convex recoloring problem. We also address a generalization of the classic vertex coloring problem. The $k$-fold coloring problem consists in covering the set of vertices of a graph into a minimum number of stable sets in such a way that every vertex is covered by at least $k$ (possibly identical) stable sets. Note that, in the context of $k$-fold colorings, a coloring can be viewed as a multiset of stable sets. The last coloring problem studied in this thesis is associated with oriented graphs. The proper orientation problem consists in orienting the edge set of a given graph so that adjacent vertices have different in-degrees and the maximum in-degree is minimized. Clearly, the in-degrees induce a partition of the vertex set into stable sets, that is, a coloring (in the conventional sense) of the vertices.

In addition to the mentioned coloring problems on graphs, we study a problem related to subdivision of directed graphs (or simply, digraphs). Given a graph $G$, the subdivision of an edge uv in $G$ is a graph operation in which $u v$ is removed from $G$, a new vertex, say $w$, and edges $w u$ and $w v$ are created. One may easily extend the concept of subdivision to digraphs. Given a digraph $D$, the subdivision of an arc uv in $D$ is a digraph operation in which $u v$ is removed from $D$, a new vertex $w$, and arcs $u w$ and $w v$ are created. Note that the directions of arcs $u w$ and $w v$ are coherent to the direction of $u v$. The graph (respectively, digraph) obtained from any sequence of subdivision operations is called a subdivision of $G$ (respectively, of $D)$.

In the eighties, Mader [56] conjectured that, for every acyclic digraph $H$, there exists an integer $f(H)$ such that every digraph with minimum out-degree at least $f(H)$ contains a subdivision of $H$ as a subdigraph. In this context, the subdivision problem consists in showing the existence of such a function for subclasses of acyclic digraphs.

We investigate the previously mentioned problems from different points of view. For the optimization problems, the focus is on the design of algorithms, polyhedral studies, and hardness issues; for the graph problems, the focus is on their structural aspects. As we shall see, the contributions of this thesis are spread over different areas.

In Part II, we address the convex recoloring problem. A coloring of the vertices of a connected graph is $r$-convex if each color class induces a subgraph with at most $r$ components. We address the $r$-convex recoloring problem defined as follows. Given a graph $G$ and a coloring of its vertices, recolor a minimum number of vertices of $G$ so that the resulting coloring is $r$-convex. This problem, known to be $\mathcal{N} \mathcal{P}$-hard even on paths, was firstly investigated on trees and for $r=1$, motivated by applications on perfect phylogenies. The more general concept of $r$-convexity, for $r \geq 2$, was proposed later, and it is also of interest in the study of protein-protein interaction networks and phylogenetic networks. In this thesis, we show that, for each $r \in \mathbb{N}$, the $r$-convex recoloring problem on $n$-vertex bipartite graphs cannot be approximated within a factor of $n^{1-\varepsilon}$ for any $\varepsilon>0$, unless $\mathcal{P}=\mathcal{N} \mathcal{P}$. We also provide strong hardness results for weighted and parametrized versions of the problem. On the positive side, we propose an integer linear formulation for the convex recoloring problem when $r=1$. Our formulation has an exponential number of variables and it is embedded in a column generation procedure in which a new column can be efficiently generated when the input graph is a tree. We provide computational experiments carried out with instances that come from an application in bioinformatics. Our column generation approach performs better than the other approaches described in the literature when the input instance has a relatively high number of colors.

The $k$-fold coloring problem is studied in Part III. A $k$-fold $x$-coloring of a graph $G$ is an assignment of (at least) $k$ distinct colors from the set $\{1,2, \ldots, x\}$ to each vertex such that any two adjacent vertices 
are assigned disjoint sets of colors. The $k$-th chromatic number of $G$, denoted by $\chi_{k}(G)$, is the smallest $x$ such that $G$ admits a $k$-fold $x$-coloring. We present an integer linear programming formulation (ILP) to determine $\chi_{k}(G)$ and study the facial structure of the corresponding polytope $\mathcal{P}_{k}(G)$. We show facets that $\mathcal{P}_{k+1}(G)$ inherits from $\mathcal{P}_{k}(G)$ and show how to lift facets from $\mathcal{P}_{k}(G)$ to $\mathcal{P}_{k+\ell}(G)$. We project facets of $\mathcal{P}_{1}\left(G \circ K_{k}\right)$ into facets of $\mathcal{P}_{k}(G)$, where $G \circ K_{k}$ is the lexicographic product of $G$ by a clique with $k$ vertices. In both cases, we can obtain facet-defining inequalities from many of those known for the 1-fold coloring polytope. We also derive facets of $\mathcal{P}_{k}(G)$ from facets of stable set polytopes of subgraphs of $G$. In addition, we present classes of facet-defining inequalities based on strongly $\chi_{k}$-critical webs and antiwebs, which extend and generalize known results for 1-fold coloring. We introduce this criticality concept and characterize the webs and antiwebs having such a property.

Part IV is devoted to the proper orientation problem. An orientation of a graph $G$ is a digraph $D$ obtained from $G$ by replacing each edge by exactly one of the two possible arcs with the same endpoints. For each $v \in V(G)$, the in-degree of $v$ in $D$, denoted by $d_{D}^{-}(v)$, is the number of arcs with head $v$ in $D$. An orientation $D$ of $G$ is proper if $d_{D}^{-}(u) \neq d_{D}^{-}(v)$, for all $u v \in E(G)$. The proper orientation number of a graph $G$, denoted by $\vec{\chi}(G)$, is the minimum of the maximum in-degree over all proper orientations of $G$. In this thesis, we prove that $\vec{\chi}(G) \leq(\Delta(G)+\sqrt{\Delta(G)}) / 2+1$ if $G$ is a bipartite graph, and $\vec{\chi}(G) \leq 4$ if $G$ is a tree. It is well-known that $\vec{\chi}(G) \leq \Delta(G)$, for every graph $G$. However, we prove that deciding whether $\vec{\chi}(G) \leq \Delta(G)-1$ is already an $\mathcal{N} \mathcal{P}$-complete problem on graphs with $\Delta(G)=k$, for every $k \geq 3$. We also show that it is $\mathcal{N} \mathcal{P}$-complete to decide whether $\vec{\chi}(G) \leq 2$, for planar subcubic graphs $G$. Moreover, we prove that it is $\mathcal{N} \mathcal{P}$-complete to decide whether $\vec{\chi}(G) \leq 3$, for planar bipartite graphs $G$ with maximum degree 5 .

Finally, we address a problem related to subdivision of digraphs in Part V. In 1985, Mader [56] conjectured the existence of a function $f$ such that every digraph with minimum out-degree at least $f(k)$ contains a subdivision of the transitive tournament of order $k$. This conjecture is still completely open, as the existence of $f(5)$ remains unknown. In this thesis, we show that if $D$ is an oriented path, or an inarborescence (that is, a tree with all edges oriented towards the root), then every digraph with minimum out-degree large enough contains a subdivision of $D$.

We remark that most of the contributions presented in this thesis result from collaborations with researchers from different universities. For each problem, we name, in section "Contributions", the researchers who collaborated in the studies of that problem. Moreover, we cite the published papers (if any) containing the corresponding results.

In the next chapter, we introduce basic concepts and notation in graph theory, polyhedral combinatorics, approximation and parameterized algorithms that are used throughout the thesis. 


\section{Chapter 2}

\section{Basic concepts, terminology and notation}

In this thesis, we use notation and terminology that are conventional in graph theory, approximation and parameterized algorithms, and polyhedral combinatorics. However, to present a self-contained text, we provide here the basic concepts, terminology and notation that appears throughout this text. The reader who is familiar with those areas may simply skip this chapter without any loss.

The terminology adopted for graphs, despite small variations, is the same as in Diestel [34], Bondy and Murty [14], and Bang-Jensen and Gutin [9]. Basic concepts on polyhedral combinatorics may be found in Ferreira and Wakabayashi [38], and in Nemhauser and Wolsey [62]. In Carvalho et al. [33] and Vazirani [77] one may find concepts and results on approximation algorithms and hardness of approximation. On topics concerning parameterized algorithms and complexity, we refer the reader to Cygan et al. [31], Flum and Grohe [39], and Downey and Fellows [36].

\subsection{Graph theory}

A graph $G$ consists of a non-empty finite set $V(G)$ of elements called vertices and a finite set $E(G)$ of unordered pairs of distinct vertices called edges, that is, $E(G) \subseteq\{\{u, v\}: u, v \in V(G)$ and $u \neq v\}$. We call $V(G)$ the vertex set and $E(G)$ the edge set of $G$, respectively. The simplified notation $V$ and $E$ is preferred when the graph is clear from the context. The complement of $G$ is denoted by $\bar{G}=(V, \bar{E})$, that is, $V(\bar{G})=V(G)$ and $E(\bar{G})=\bar{E}(G)=\{\{u, v\}: u, v \in V(G)$ and $u \neq v\} \backslash E(G)$.

Two different vertices $u$ and $v$ in $G$ are adjacent or neighbors if there exists an edge incident to $u$ and $v$; we denote such an edge by $\{u, v\}$ (or just $u v$ ). We say that $u$ and $v$ are the endpoints, or endvertices of edge $u v$. We also say that a vertex $v$ is incident to an edge $e$ if $v$ is one of the endpoints of $e$. The set of neighbors of a vertex $v \in V(G)$ is denoted by $N(v)$. More generally, given $W \subseteq V(G)$, we denote by $N(W)$ the set of neighbors in $V(G) \backslash W$ of vertices in $W$, that is, $N(W):=\bigcup_{v \in W} N(v) \backslash W$ is the neighborhood of $W$. The complement of $N(W) \cup W$ is called anti-neighborhood of $W$ and is denoted by $\bar{N}(W)$.

Let $G$ be an undirected graph. A subset $S \subseteq V(G)$ is said to be stable if all vertices are pairwise non-adjacent, that is, $u v \notin E(G)$ for all $u, v \in S$. The stability number $\alpha(G)$ is the size of the largest stable set of $G$. Reciprocally, a clique in $G$ is a subset $K \subseteq V(G)$ such that all vertices are pairwise adjacent. The clique number of $G$, which is denoted by $\omega(G)$, is the size of the largest clique of $G$. For 
simplicity, we often refer to a graph itself as being a clique or a stable when its set of vertices is a clique or a stable set, respectively.

If $G$ and $G^{\prime}$ are two graphs such that $V\left(G^{\prime}\right) \subseteq V(G)$ and $E\left(G^{\prime}\right) \subseteq E(G)$, then $G^{\prime}$ is said to be a subgraph of $G$, and we write $G^{\prime} \subseteq G$. If $G^{\prime} \subseteq G$ and $G^{\prime}$ contains all edges $u v \in E(G)$ with $u, v \in V\left(G^{\prime}\right)$, then $G^{\prime}$ is called an induced subgraph of $G$. In this case, we say that $V\left(G^{\prime}\right)$ induces $G^{\prime}$ in $G$ and we write $G^{\prime}:=G\left[V\left(G^{\prime}\right)\right]$. Thus, if $U \subseteq V(G)$, then $G[U]$ denotes a subgraph of $G$ whose set of vertices is $U$ and the edges are precisely those edges of $G$ with both endpoints in $U$. If $W \subseteq V(G)$, then $G-W$ denotes the subgraph obtained from $G$ by removing $W$, that is, $G-W=G[V(G) \backslash W]$. For simplicity, if $v$ is a vertex of $G$, then $G-v$ denotes the subgraph $G-\{v\}$.

A path is a non-empty graph $P$ with vertex set $V(P)=\left\{v_{0}, v_{1}, \ldots, v_{n-1}\right\}$ and edge set $E(P)=$ $\left\{v_{0} v_{1}, \ldots, v_{n-2} v_{n-1}\right\}$, where the $v_{i}^{\prime} s$ are all different. We say that $v_{0}$ and $v_{n-1}$ are linked by $P$, and we call them the extremities of $P$. Vertices $v_{1}, v_{2}, \ldots, v_{n-2}$ are the internal vertices of $P$. The number of edges of a path is its length. Frequently, we refer to a path by the natural sequence of its vertices, that is, $P=v_{0} v_{1} \ldots v_{n-1}$, and we say that $P$ is a path from $v_{0}$ to $v_{n-1}$. Given integers $i$ and $j$ such that $0 \leq i \leq j \leq n-1$, we denote by $P v_{i}$ the subpath of $P$ from $v_{0}$ to $v_{i}$, that is, $P v_{i}:=v_{0} \ldots v_{i}$. Analogously, we write $v_{i} P:=v_{i} \ldots v_{n-1}$ and $v_{i} P v_{j}:=v_{i} \ldots v_{j}$. We use the following similar and intuitive notation for the concatenation of paths. For instance, if the union $P u \cup u Q v \cup v R$ of three paths is also a path, then we denote it by $P u Q v R$. Given a graph $G$ and two paths $P=v_{0} \ldots v_{k} \subseteq G$ and $P^{\prime}=z_{0} \ldots z_{l} \subseteq G$, we denote by $P P^{\prime}$ the path in $G$ that corresponds to the concatenation of $P$ and $P^{\prime}$ if $V(P) \cap V\left(P^{\prime}\right)=\emptyset$ and $v_{k} z_{0} \in E$. The graph $\left(V(P), E(P) \cup\left\{v_{0}, v_{n-1}\right\}\right)$, where $P=v_{0} \ldots v_{n-1}$ is a path, is called a cycle.

A graph $G$ is said to be connected if there exists a path linking any pair of vertices in $G$. A tree is a connected graph with no cycle.

A directed graph (or simply digraph) $D$ consists of a non-empty finite set $V(D)$ of vertices and a finite set $A(D)$ of ordered pairs of distinct vertices called arcs. We call $A(D)$ the arc set of $D$.

For an $\operatorname{arc}(u, v)$ the first vertex $u$ is its tail and the second vertex $v$ is its head. We also say that the arc $(u, v)$ leaves $u$ and enters $v$. The head and the tail of an arc are its endpoints or endvertices; we say that the endpoints are adjacent, that is, $u$ is adjacent to $v$ and $v$ is adjacent to $u$. If $(u, v)$ is an arc, we also say that $u$ dominates $v$ (or $v$ is dominated by $u$ ) and denote it by $u \rightarrow v$. We say that a vertex $u$ is incident to an $\operatorname{arc} a$ if $u$ is the head or tail of $a$. We often denote an arc $(u, v)$ by $u v$.

The above definition of a digraph implies that digraphs may have arcs with the same endpoints, but they do not contain parallel (also called multiple) arcs, that is, pairs of arcs with the same tail and the same head, or loops (i.e. arcs whose head and tail coincide).

Let $D$ be a digraph. For every vertex $v \in V(D)$, the out-neighborhood of $v$ is the set $N_{D}^{+}(v)=\{u \in$ $V(D): v u \in A(D)\}$, its in-neighborhood is $N_{D}^{-}(v)=\{u \in V(D): u v \in A(D)\}$, and its neighborhood is $N_{D}(v)=N_{D}^{+}(v) \cup N_{D}^{-}(v)$. The vertices in $N_{D}^{+}(v)$ are called the out-neighbors of $v$, the ones in $N_{D}^{-}(v)$ are the in-neighbors of $v$, and the vertices in $N_{D}(v)$ are the neighbors of $v$. The out-degree of $v$, denoted by $d_{D}^{+}(v)$, is the number of arcs in $D$ with tail $v$, that is, $d_{D}^{+}(v)=\left|N_{D}^{+}(v)\right|$. Similarly, The in-degree of $v$ is $d_{D}^{-}(v)=\left|N_{D}^{-}(v)\right|$.

The minimum out-degree and minimum in-degree of $D$ are, respectively,

$$
\delta^{+}(D)=\min \left\{d_{D}^{+}(v): v \in V(D)\right\} \text { and } \delta^{-}(D)=\min \left\{d_{D}^{-}(v): v \in V(D)\right\}
$$

Similarly, one may define the maximum out-degree, $\Delta^{+}(D)$, and the maximum in-degree, $\Delta^{-}(D)$.

For degrees, neighborhoods as well as for other parameters and sets of digraphs, we usually omit the subscript for the digraph when it is clear from the context. 
A digraph $H$ is a subdigraph of a digraph $D$ if $V(H) \subseteq V(D), A(H) \subseteq A(D)$, and every arc in $A(H)$ has both endpoints in $V(H)$. We say that $H$ is a spanning subdigraph (or a factor) of $D$ if $V(H)=V(D)$. If every arc of $A(D)$ with both endpoints in $V(H)$ belongs to $A(H)$, we say that $H$ is induced by $X=V(H)$ (we write $H=D[X]$ ), and call $H$ an induced subdigraph of $D$.

\subsection{Approximation algorithms}

An optimization problem has three main ingredients: a set of instances, a set of feasible solutions $\operatorname{sol}(I)$ for each instance $I$, and a function which assigns a rational number val $(S)$ to every feasible solution $S$. The number $\operatorname{val}(S)$ is the value of $S$. When the set $\operatorname{sol}(I)$ of feasible solutions associated with an instance $I$ is empty, we say that this instance is infeasible; otherwise, the instance is feasible.

In a minimization problem, we are interested in finding feasible solutions of minimum value, whereas in a maximization problem the aim is to find feasible solutions of maximum value. When one of the two optimization senses - minimum or maximum - is implicit, we simply say an optimization problem and an optimal value. The value of every optimal solution for an instance $I$ is denoted by opt $(I)$. Hence, $\operatorname{opt}(I):=\operatorname{val}\left(S^{*}\right)$, where $S^{*}$ is an optimal solution for $I$. Obviously, this value is well defined only if $I$ is feasible.

Each instance $I$ of a given optimization problem is assigned a natural number $\langle I\rangle$ called the size of $I$. We can imagine that the instances, as well as the feasible solutions, are strings of characters. Hence, $\langle I\rangle$ is the length of the string of characters corresponding to $I$. An algorithm $\mathcal{A}$ for an optimization problem is said to be polynomial-time (or simply polynomial) if there exists a polynomial function $p$ such that the running time of $\mathcal{A}$ is bounded from above by $p(\langle I\rangle)$ for every instance $I$. The definition of polynomial algorithms must be understood as a formalization of the concept of efficient algorithms. If a problem is $\mathcal{N} \mathcal{P}$-hard, then it is unlikely that there exists a polynomial exact algorithm for this problem.

Consider now an optimization problem $\Pi$ such that $\operatorname{val}(S)>0$ for every feasible solution $S$ of all instances of $\Pi$. Let $\mathcal{A}$ be a polynomial-time algorithm such that, for every feasible instance $I$ of $\Pi$, produces a feasible solution $\mathcal{A}(I)$ of $I$. If $\Pi$ is a minimization problem and

$$
\operatorname{val}(\mathcal{A}(I)) \leq \alpha \operatorname{opt}(I)
$$

holds for every instance $I$ of $\Pi$, we say that $\mathcal{A}$ is an $\alpha$-approximation for problem $\Pi$. The factor $\alpha$ may be a function that depends on $I$. We say that $\alpha=\alpha(I)$ is the approximation ratio of the algorithm. It is clear that $\alpha \geq 1$ since $\Pi$ is a minimization problem. In the case of maximization problems, we replace $(2.1)$ by

$$
\operatorname{val}(\mathcal{A}(I)) \geq \alpha \operatorname{opt}(I)
$$

Clearly, we now have $0<\alpha \leq 1$. An approximation algorithm is an $\alpha$-approximation for some $\alpha$. A 1 approximation for an optimization problem is an exact algorithm for the problem.

Observe that an algorithm $\mathcal{A}$ is an $\alpha$-approximation for a minimization (maximization) problem if $\alpha$ is an upper (lower) bound for the ratio between $\operatorname{val}(\mathcal{A}(I))$ e opt $(I)$ of every instance $I$ of the problem. Since the value opt $(I)$ is, in general, as difficult to compute as finding an optimal solution for $I$, to demonstrate that an algorithm is an $\alpha$-approximation it is essential to have good bounds for opt $(I)$, for every feasible instance $I$. In the context of optimization problems, we assume opt $(I)>0$ for every instance $I$. 


\subsection{Parameterized algorithms}

A parameterized problem (also known as parameterized language) is a subset $\Pi$ of $\Sigma^{*} \times \mathbb{N}$, where $\Sigma$ is a fixed finite alphabet. Given an instance $(I, p) \in \Sigma^{*} \times \mathbb{N}$ of a parameterized problem $\Pi, I$ is called the main part of the instance and $p$ is the parameter of the instance. We say that a parameterized problem $\Pi$ is fixed-parameter tractable (FPT) if there exists a computable function $f: \mathbb{N} \rightarrow \mathbb{N}$ and an algorithm that decides whether $(I, p) \in \Pi$ in $f(p) \cdot n^{\ell}$ steps, where $n=|\langle I, p\rangle|$ is the size of the instance, $f$ depends only on $p$, and $\ell$ is a constant independent of $n$ and $p$. Such an algorithm is called FPT. The class of all fixed-parameter tractable problems is called $\mathcal{F P T}$.

The complexity classes of parameterized problems, as well as the conventional complexity classes, have three basic ingredients. Firstly, we need a notion of easiness which is given by the definition of the $\mathcal{F P T}$ class. Secondly, it is required a concept of reduction between parameterized problems. Finally, we need some canonical problems that are believed to be intractable.

From the definition of fixed-parameter tractable problems, it naturally follows a concept of reduction that expresses the fact the two parameterized problems have a comparable parameterized complexity. In other words, if a parameterized problem $\Pi_{1}$ may be reduced to a parameterized problem $\Pi_{2}$, and $\Pi_{2}$ is in $\mathcal{F P} \mathcal{T}$, then $\Pi_{1}$ also belongs to $\mathcal{F P} \mathcal{P}$. Formally, a parameterized reduction from a problem $\Pi_{1}$ to a problem $\Pi_{2}$, denoted by $\Pi_{1} \preceq_{\mathrm{FPT}} \Pi_{2}$, is an algorithm that computes, from an instance $(I, p)$, an instance $\left(I^{\prime}, p^{\prime}\right)$ such that

1. $(I, p)$ is a YES-instance of $\Pi_{1}$ if and only if $\left(I^{\prime}, p^{\prime}\right)$ is a YES-instance of $\Pi_{2}$,

2. $p^{\prime} \leq g(p)$ for some computable function $g$ which only depends on $p$, and

3. the computation is done in time $f(p) \cdot n^{\ell}$, where $n$ is the size of the instance (i.e. $\left.n=|\langle I, p\rangle|\right), p$ is a parameter, $\ell$ is a constant independent of $n$ and $p$, and $f$ is any computable function that only depends on $p$.

Theorem 2.1 (Cygan et al. [31]). Let $\Pi_{2} \in \mathcal{F P T}$. If $\Pi_{1} \preceq_{\mathrm{FPT}} \Pi_{2}$, then $\Pi_{1} \in \mathcal{F} \mathcal{P} \mathcal{T}$.

Note that item (2) of the previous definition requires something that does not necessarily hold for the classic polynomial reductions: the parameter of the constructed instance has to be bounded by some function on the parameter of the original instance. Hence, it is not true that every $\mathcal{N} \mathcal{P}$-completeness proof implies the existence of a parameterized reduction. Therefore, in general, parameterized reductions and polynomial reductions are incomparable. However, most of the parameterized reductions are indeed polynomial reductions.

Downey and Fellows [36] introduced the concept of $\mathcal{W}$-hierarchy in an attempt to capture the exact complexity of several hard parameterized problems. We next present the fundamental definitions related to this hierarchy.

A boolean circuit is an acyclic directed graph whose vertices are labeled as follows:

- every vertex of in-degree 0 is an input vertex,

- every vertex of in-degree 1 is a negation vertex, and

- every vertex of in-degree at least 2 is either an and-vertex or an or-vertex.

Additionally, exactly one of the vertices of out-degree 0 is labeled as the output-vertex (in addition to being, e.g., an and-vertex). The depth of a circuit is the length of the longest directed path from an input-vertex to the output-vertex. 
Given an assignment from the values TRUE and FALSE to every input-vertex of a boolean circuit, it is possible to determine the value (TRUE or FALSE) of each vertex of the circuit. Particularly, if the value of the output-vertex is TRUE, then we say that the corresponding assignment satisfies the circuit.

Given a circuit $C$ and an integer $p$, the Weighted Circuit Satisfiability (WCS) problem consists in deciding whether $C$ admits an assignment satisfying it and has weight $p$, where the weight of an assignment is the number of input vertices that receive value TRUE.

The levels of the $\mathcal{W}$-hierarchy are defined by restricting WCS to different classes of circuits. Formally, if $\mathcal{C}$ is a class of boolean circuits, then we define $\operatorname{WCS}[\mathcal{C}]$ as the restriction of the problem where every input circuit belongs to the class $\mathcal{C}$. In what follows, we specify the classes of circuits that are used to define the levels of the $\mathcal{W}$-hierarchy.

The vertices of a circuit are distinguished into small vertices, the vertices that have in-degree at most 2 , and large vertices, the vertices with in-degree greater than 2 . The weft of a circuit is the maximum number of large vertices in a path from an input-vertex to the output-vertex. We denote by $\mathcal{C}_{t, d}$ the class of boolean circuits of weft at most $t$ and depth at most $d$.

Let $t$ be a positive integer. A parameterized problem $\Pi$ belongs to class $\mathcal{W}[t]$ if there exists a parameterized reduction from $\Pi$ into the problem $\operatorname{WCS}\left[\mathcal{C}_{t, d}\right]$, for some $d>1$.

\subsection{Polyhedral combinatorics}

Unless stated otherwise, all vectors considered in this text are column vectors. Hence, if $x$ is a vector, then $x^{T}$ denotes its transpose which is a row vector. The inner product of two vectors $x, y \in \mathbb{R}^{n}$ is the scalar $x^{T} y:=\sum_{i=1}^{n} x_{i} y_{i}$. A vector such that all its entries are equal to 1 is denoted by 1 . A vector such that all its entries are equal to 0 is denoted by $\mathbf{0}$. The norm of a vector $x \in \mathbb{R}^{n}$, denoted by $|x|$, is $\sqrt{\sum_{i=1}^{n} x_{i}^{2}}$. A vector is said to be a unit vector if its norm is equal to 1 . We use the notation $e^{i}$ to refer to a the unit vector (of the canonical basis) such that all coordinates are null but its $i$-th coordinate which is equal to 1 .

Let $S \subseteq \mathbb{R}^{n}$ be a non-empty set. A vector $x \in \mathbb{R}^{n}$ is a linear combination of vectors $x^{1}, x^{2}, \ldots, x^{t}$ in $S$ if $x=\sum_{i=1}^{t} \lambda_{i} x^{i}$ for some $\left(\lambda_{1}, \lambda_{2}, \ldots, \lambda_{t}\right) \in \mathbb{R}^{t}$. Such a linear combination is called affine if $\lambda_{1}+\ldots+\lambda_{t}=1$; conic if $\lambda_{1}, \ldots, \lambda_{t} \geq 0$; and convex if it is affine and conic. We denote by $\operatorname{span}(S)$ the linear hull of the elements in $S$, that is, the set of all vectors that can be obtained from some linear combination of a finite number of vectors in $S$. Analogously, we define the affine, conic and convex hull of $S$, denoted by affine $(S), \operatorname{conic}(S)$ and $\operatorname{conv}(S)$, respectively.

Let $S \subseteq \mathbb{R}^{n}$ be a set with $k$ vectors such that $S=\left\{x^{1}, \ldots, x^{k}\right\}$. We say that $S$ is linearly independent if the single solution for $\sum_{i=1}^{k} \lambda_{i} x^{i}=0$ is $\lambda=\mathbf{0}$. We say that $S$ is affinely independent if the single solution for $\sum_{i=1}^{k} \lambda_{i} x^{i}=0, \sum_{i=1}^{k} \lambda_{i}=0$ is $\lambda=\mathbf{0}$.

Consider now a set $S \subseteq \mathbb{R}^{n}$. The rank of $S$, denoted by $\operatorname{rank}(S)$, is the cardinality of a largest linearly independent subset of $S$. Analogously, the affine-rank of $S$, denoted by affine-rank $(S)$, is equal to the cardinality of a largest subset of $S$ which is affinely independent. It is folklore that for every $S \subseteq \mathbb{R}^{n}$ we have affine-rank $(S)=\operatorname{rank}(S)+1$ if $\mathbf{0} \in \operatorname{affine}(S)$; and affine-rank $(S)=\operatorname{rank}(S)$ otherwise. The dimension $\operatorname{dim}(S)$ of $S$ is defined as $\operatorname{dim}(S)=\operatorname{affine-rank}(S)-1$. We say that $S$ is full-dimensional if $\operatorname{dim}(S)=n$.

The rank of a matrix $A \in \mathbb{R}^{m \times n}$, denoted by $\operatorname{rank}(A)$, is the rank of the set of column vectors of $A$, which is proven to be equal to the rank of the set of row vectors of $A$. We say that $A$ has full row rank if $\operatorname{rank}(A)=m$; and full column rank if $\operatorname{rank}(A)=n$. 
A polyhedron $\mathcal{P} \subseteq \mathbb{R}^{n}$ is a set of vectors that satisfy a finite number of linear inequalities, that is, $\mathcal{P}=\left\{x \in \mathbb{R}^{n} \mid A x \leq b\right\}$, where $(A, b)$ is a matrix $m \times(n+1)$.

Throughout this thesis, we only consider polyhedra such that the corresponding matrix $(A, b)$ is rational. An inequality $\lambda x \leq \lambda_{0}$ (or simply, $\left(\lambda, \lambda_{0}\right)$ ) is said to be valid for $\mathcal{P}$ if it is satisfied by all vectors in $\mathcal{P}$, that is, $\lambda x \leq \lambda_{0}$ for all $x \in \mathcal{P}$. If $\left(\lambda, \lambda_{0}\right)$ is a valid inequality for $\mathcal{P}$ and $F=\left\{x \in \mathcal{P}: \lambda x=\lambda_{0}\right\}$, then $F$ is a face of $\mathcal{P}$. A face $F$ of $\mathcal{P}$ is called facet if $\operatorname{dim}(F)=\operatorname{dim}(\mathcal{P})-1$. 


\section{Part II}

\section{Convex recoloring}





\section{Chapter 3}

\section{The convex recoloring problem}

In this chapter, we present the basic definitions, applications, known results and an overview of our contributions related to variants of the convex recoloring problem. We remark that all graphs considered in this part are connected, finite, undirected and simple.

\subsection{Basic definitions}

A total coloring ${ }^{1}$ of a graph $G$ is a function $C$ that assigns to each vertex in $V(G)$ a color, that is, $C: V(G) \rightarrow \mathcal{C}$, where $\mathcal{C}$ is a set of colors. A partial coloring (or simply, a coloring) of $G$ is a function $C$ : $V(G) \rightarrow \mathcal{C} \cup\{\emptyset\}$, where $\emptyset$ indicates the absence of color. We say that a vertex $v$ in $G$ is uncolored if $C(v)=\emptyset$, and we say that $G$ is $k$-colored if its coloring uses $k$ colors. For each color $c \in \mathcal{C}$, the color class $c$ (denoted by $C^{-1}(c)$ ) is the set of vertices in $G$ that have color $c$, that is, $C^{-1}(c):=\{v \in V(G): C(v)=c\}$. Note that the coloring defined here differs from the classical vertex coloring, in which adjacent vertices are required to have different colors.

A totally colored graph is a pair $(G, C)$ consisting of a graph $G$ and a total coloring $C$ of its vertices. We say that a total coloring $C$ is $r$-convex if, for each $c \in \mathcal{C}$, the color class $c$ induces a subgraph of $G$ with at most $r$ components. A (partially) colored graph is defined similarly. An $r$-convex (partial) coloring $C: V(G) \rightarrow \mathcal{C} \cup\{\emptyset\}$ is a (partial) coloring that can be extended to an $r$-convex total coloring by solely assigning a color in $\mathcal{C}$ to each uncolored vertex. A good coloring is a coloring such that, for each $c \in \mathcal{C}$, the color class $c$ induces a connected subgraph of $G$. Note that we can extend every good coloring of $G$ to a convex total coloring in time $\mathcal{O}(|V(G)|+|E(G)|)$. Given a colored graph $(G, C)$, any other coloring $C^{\prime}: V(G) \rightarrow \mathcal{C} \cup\{\emptyset\}$ is a recoloring of $(G, C)$, where $\mathcal{C}$ is the same set of colors of the initial coloring $C$. We say that a vertex $v$ is recolored (by $C^{\prime}$ ) if $C(v) \neq \emptyset$ and $C(v) \neq C^{\prime}(v)$.

Next, we formally define the problem we address here, namely the $r$-Convex Recoloring. This problem was introduced by Chor et al. [27] in 2007.

Problem 3.1. $r$-Convex ReColoring $(r$-CR)

Instance: A connected graph $G$, a coloring $C: V(G) \rightarrow \mathcal{C} \cup\{\emptyset\}$ and a weight function $w: V(G) \rightarrow \mathbb{Q}_{\geq}$. Find: An r-convex recoloring $C^{\prime}$ of $(G, C)$.

GoAL: Minimize $\sum_{v \in R_{C}\left(C^{\prime}\right)} w(v)$, where $R_{C}\left(C^{\prime}\right):=\left\{v \in V(G): C(v) \neq \emptyset\right.$ and $\left.C(v) \neq C^{\prime}(v)\right\}$ is the set of vertices recolored by $C^{\prime}$.

\footnotetext{
${ }^{1}$ We remark that the term "total coloring" is also used, with a different meaning, in the context of colorings on the vertices and edges of a graph.
} 
We start with some remarks on the $r$-CR problem. We may (and we will) assume that the weight of any uncolored vertex is 0 . If the function $w$ only assigns weights 1 or 0 to the vertices of $G$, then we get a particular problem that we call $r$-CR-BIN. More formally, $r$-CR-BIN is the $r$-CR problem restricted to instances $(G, C, w)$ such that, for each $v \in V(G), w(v)=1$ if $C(v) \neq \emptyset$; and $w(v)=0$, otherwise. For simplicity, we denote by $(G, C)$ an instance of this problem, which can be seen as a cardinality (or unweighted) version of $r$-CR.

\subsection{Applications}

The $r$-CR problem is of interest for applications related to protein-protein interaction networks and phylogenetic networks [27]. Additionally, when $r=1, r$-CR is used in the contexts of routing problems, transportation networks [47] and phylogenetic trees [59].

The 1-CR problem was firstly investigated by Moran and Snir in 2005, motivated by its application in the study of phylogenetic trees. Given a set of species, a phylogenetic tree is a colored tree representing the course of evolution of these species. Its leaves correspond to the given set of species and its internal vertices correspond to extinct species. The colors of the vertices correspond to character states, where a character (coloring) is a biological attribute shared among all the species, and a character state (color) is the state of this character shown by a species (vertex). A natural assumption in Biology is that the reconstructed phylogenetic tree has the property that each of the characters should have evolved without reverse or convergent transitions. These concepts are captured, in graph theoretical terms, by the requirement that each of the color classes has to induce a connected subgraph.

Moran and Snir [61] provide a detailed discussion about the important role played by convexity in the study of phylogenetic trees.

\subsection{Known results}

The $r$-CR problem (with $r \geq 2$ ) was introduced by Chor et al. in 2007 [27]. In their work, they investigate a closely related problem called $r$-Connected Coloring Completion ( $r$-CCC): given a colored graph, it asks whether it is possible to extend the initial coloring to a total $r$-convex coloring. Chor et al. [27] proved that 1-CCC is $\mathcal{N} \mathcal{P}$-hard even if the colored graph has only two colors. Moreover, they showed that $r$-CCC, parameterized by the treewidth, is $\mathcal{W}[1]$-hard for all $r \geq 2$, and fixed-parameter tractable for $r=1$.

The 1-CR (or simply CR) problem has received considerable attention in the last years. We mention some results for totally colored graphs. Kanj and Kratsch [48] proved that this problem is $\mathcal{N} \mathcal{P}$-hard for paths even if each color appears at most twice. Recently, Campêlo et al. [21] showed that the CR-BIN problem is $\mathcal{N} \mathcal{P}$-hard on 2-colored grids. Moreover, they proved that CR-BIN parameterized by the number of color changes is $\mathcal{W}[2]$-hard on general graphs. Approximation algorithms for the unweighted totally colored case have also been designed: with ratio $(2+\varepsilon)$ for bounded treewidth graphs [47], ratio 2 for paths [60], and ratio $3 / 2$ for paths in which each color appears at most twice [54]. More recently, BarYehuda et al. [10] designed a 3/2-approximation algorithm for general graphs in which each color appears at most twice. Their algorithm yields a 5/4-approximation for paths, improving the approximation ratio 3/2 obtained by Lima and Wakabayashi [54]. On the other hand, Campêlo et al. [21] showed that 1-CR-BIN (or simply CR-BIN) cannot be approximated within a ratio of $\mathcal{O}(\log n)$ on $n$-vertex $k$-colored bipartite graphs, unless $\mathcal{P}=\mathcal{N} \mathcal{P}$, for each $k \geq 2$. 
An integer linear formulation for $\mathrm{CR}$ on general graphs was introduced by Campêlo et al. in [19]. A detailed polyhedral study was presented and computational experiments, carried out with relatively large size instances that come from an application in phylogenetic trees (that is, the input graph is a tree), showed that the problem can be solved in reasonable running time. Recently, a more compact formulation for CR on trees was proposed by Chopra et al. in [26]. They showed that their ILP formulation dominates the one described in [19]. Moreover, the computational experiments presented in [26] showed that the later formulation outperformed the former one.

\subsection{Contributions}

Our aim in the study of convex recoloring problems is twofold. On the theoretical side, we prove inapproximability and $\mathcal{W}[2]$-hardness results which provide a better understanding of the difficulty associated with the various convex recoloring problems that have been treated in the literature. On the applied side, we propose an integer linear formulation for $\mathrm{CR}$ on general graphs and we design an algorithm to tackle the CR problem on trees. We provide computational experiments on instances that come from an application on phylogenetic trees and show that our solution method (on input instances with many colors) performs better than the best solving method described in the literature so far.

The remainder of this part of the thesis is organized as follows. In the next chapter, we show two inapproximability results, under the hypothesis that $\mathcal{P} \neq \mathcal{N} \mathcal{P}$. Namely, we prove that for every $r$ and $k \geq 2$, the $r$-CR problem on $k$-colored bipartite graphs admits no exponential-factor approximation algorithm, and the $r$-CR-BIN problem on such $n$-vertex graphs does not admit an $n^{1-\varepsilon}$-approximation, where $\varepsilon$ is an arbitrary real number. The last result not only dramatically improves the $\mathcal{O}(\log n)$ approximation threshold obtained by Campêlo et al. [21], but it is also the strongest possible. Moreover, we prove that the decision version of the $r$-CR-BIN problem parameterized by the number of color changes (that is, the standard parameterization of $r$-CR-BIN) is $\mathcal{W}$ [2]-hard even on $k$-colored bipartite graphs (for every $r$ and $k \geq 2$ ). Thus, it is unlikely that there exists an FPT-algorithm for this problem. This result, which is presented in Chapter 4, generalizes (by means of a different reduction) the results of Campêlo et al. [21] on the $r$-CR-BIN problem.

In Chapter 5, we propose a new formulation for the CR problem on general graphs. Our formulation is embedded in a column generation procedure, in which a new column can be efficiently generated when the input graph is a tree. More precisely, we present an algorithm for generating a new column in time $\mathcal{O}\left(k n^{2}\right)$, where $k$ and $n$ are, respectively, the number of colors and vertices of the input colored tree. Although our formulation has a huge number of variables, in practice generating only a few variables may be enough to solve the problem. In Section 5.3, we show a mapping of the polytope associated with the linear relaxation of our formulation which is contained in the polytope associated with the linear relaxation of the formulation proposed by Chopra et al. [26].

We provide computational experiments for the $\mathrm{CR}$ problem on trees, carried out with the same type of instances used in [19, 26], to compare the proposed formulation with the one introduced by Chopra et al. in [26]. The computational experiments, which are presented in Section 5.4, show that our column generation approach performs better than the solving method proposed in [26] when the input instance has many colors.

We remark that the results in Chapter 5 were obtained in collaboration with Alexandre Freire from Universidade de São Paulo. 


\section{Chapter 4}

\section{Hardness results}

In this chapter, we prove hardness results which provide a better understanding of the difficulty associated with the various convex recoloring problems that have been treated in the literature. First, we prove strong inapproximability results for the weighted and unweighted versions of the convex recoloring problem. Then, we study the hardness of a parameterized version of the unweighted convex recoloring problem.

\subsection{Approximation thresholds}

In the next proofs, we present polynomial time reductions from the $r$-Connected Coloring Completion ( $r$-CCC) problem which was introduced by Chor et al. [27].

Problem 4.1. $r$-Connected Coloring Completion $(r$-CCC) InSTANCE: $A$ graph $G$ and a coloring $C: V(G) \rightarrow \mathcal{C} \cup\{\emptyset\}$.

QUESTION: Is $C$ an $r$-convex coloring of $G$ ? That is, does there exist an r-convex total coloring $C^{\prime}: V(G) \rightarrow$ C of $G$ such that, for each $v \in V(G), C^{\prime}(v)=C(v)$ if $C(v) \neq \emptyset$; and, for each $c \in \mathcal{C}$, the subgraph induced by the color class $c$ has at most $r$ components?

For every positive integer $r$, we assume that the graph in each instance $I$ of $r$-CCC has more than $r$ vertices. Otherwise, $I$ is trivially a YES-instance of $r$-CCC. When $r=1$, we also assume that the coloring of the input graph in $I$ is not good. Otherwise, $I$ is a Yes-instance and it is easy to compute a convex total coloring that extends the given one.

Chor et al. [27] proved that the 1-CCC problem is $\mathcal{N} \mathcal{P}$-complete on 2-colored bipartite graphs. Inspired by the proof presented by these authors, we may prove the following theorem by using the gadgets in Figure 4.1 to show a reduction from 3 -SAT.

Theorem 4.2. For every $r$ and $k \geq 2$, the $r$-CCC problem is $\mathcal{N} \mathcal{P}$-complete on $k$-colored bipartite graphs.

Despite being a slight generalization of the result due to Chor et al., Theorem 4.2 is essential for the proofs of inapproximability and parameterized intractability presented in this thesis, as we shall see next.

Campêlo et al. [21] proved that the CR-BIN restricted to instances with $k$ colors cannot be approximate within $\alpha \ln n$ on $n$-vertex bipartite graphs, where $\alpha$ is a constant, unless $\mathcal{P}=\mathcal{N} \mathcal{P}$. In what follows, we show a much stronger inapproximability result for $r$-CR-BIN that also holds for $k$-colored $n$-vertex bipartite graphs. Namely, we prove that this problem is hard to be approximated within $n^{1-\varepsilon}$ for every positive $\varepsilon$, 


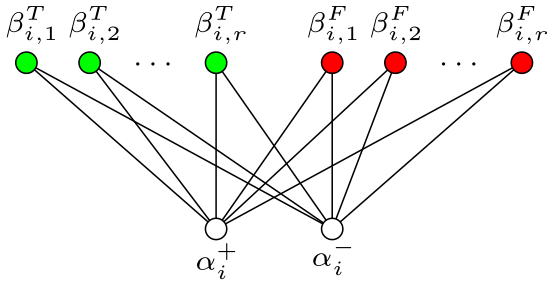

(a) Gadget associated with variable $x_{i}$.

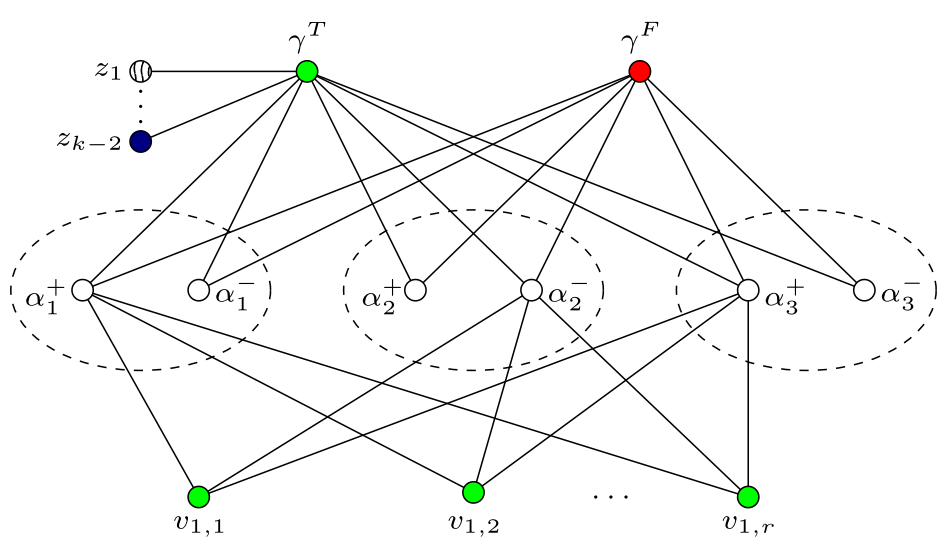

(b) Graph obtained from formula $\left(x_{1} \vee \neg x_{2} \vee x_{3}\right)$. The dashed ellipse represents the corresponding variable gadget.

Figure 4.1: Gadgets used in the proof of Theorem 4.2.

unless $\mathcal{P}=\mathcal{N} \mathcal{P}$. This result implies that it is unlikely that there exists an approximation algorithm with ratio smaller than the ratio of a trivial algorithm that simply assigns the same color to each vertex.

In what follows, for each integer $t$, we define $[t]=\{1,2, \ldots, t\}$ if $t \geq 1$, and $[t]=\emptyset$ otherwise.

Theorem 4.3. For every $r$ and $k \geq 2$, and every positive real $\varepsilon<1$, there is no $N^{1-\varepsilon}$-approximation for the $r$-CR-BIN problem on $k$-colored $N$-vertex bipartite graphs, unless $\mathcal{P}=\mathcal{N} \mathcal{P}$.

Proof. Suppose that there exists a polynomial-time approximation algorithm $\mathcal{A}$ for the $r$-CR-BIN problem on $k$-colored $N$-vertex bipartite graphs with ratio $N^{1-\varepsilon}$, for some $\varepsilon>0$. Consider such an $\varepsilon$, and let $\ell$ be the smallest integer such that $\frac{\ell}{\ell+4} \geq 1-\varepsilon$. We define the function $f(x)=x^{\ell}$.

Let $I=(G, C)$ be an instance of 1-CCC, where $G$ is a bipartite graph with $n$ vertices and $C: V(G) \rightarrow$ $\left\{\emptyset, T, F, c_{1}, \ldots, c_{k-2}\right\}$ is a coloring with $k$ colors. If $n \leq \max \{r, k\}$, then one may determine in constanttime whether $I$ is a YES-instance of 1-CCC since $r$ and $k$ are constants. Henceforth, let us assume that $n>$ $\max \{r, k\}$. Suppose that each color in the color set $\left\{T, F, c_{1}, \ldots, c_{k-2}\right\}$ appears at least once in $(G, C)$. Thus, $n \geq 3$ since $G$ has at least one uncolored vertex. From instance $I$, we build an instance $I^{\prime}=\left(G^{\prime}, C^{\prime}\right)$ of the $r$-CR-BIN problem as follows.

First, we show how to construct the graph $G^{\prime}$ from $G$. Let $W=\{v \in V(G): C(v) \neq \emptyset\}$ be the set of colored vertices in $(G, C)$. For each $v \in W$, we add to $G$ a set $S(v)$ containing $2 \cdot n f(n)+r$ new vertices and we make each vertex in $S(v)$ adjacent to $v$. Moreover, for each $v \in V(G) \backslash W$, we add to $G$ two vertices $v_{T}, v_{F}$ and two edges $v v_{T}, v v_{F}$. These additional vertices are meant to eliminate feasible solutions for $I^{\prime}$ which have value 0 .

Consider now two vertices $w_{T}, w_{F} \in W$ such that $C\left(w_{T}\right)=T$ and $C\left(w_{F}\right)=F$. For every $i \in[k-2]$ and $j \in[r-1]$, we add to $G$ a vertex $z_{i, j}$ adjacent to $w_{T}$, a set $S\left(z_{i, j}\right)$ with $2 \cdot n f(n)+r$ vertices and we make each vertex in this set adjacent to $z_{i, j}$. In addition, for every $j \in[r-1]$, we add to $G$ a vertex $z_{F, j}$ adjacent to $w_{T}$ and a vertex $z_{T, j}$ adjacent to $w_{F}$. Finally, for every $j \in[r-1]$, we add two sets $S\left(z_{T, j}\right)$ and $S\left(z_{F, j}\right)$, each one containing $2 \cdot n f(n)+r$ vertices, and we make each vertex in $S\left(z_{T, j}\right)$ adjacent to $z_{T, j}$ and each one in $S\left(z_{F, j}\right)$ adjacent to $z_{F, j}$. Figure 4.2 shows the neighborhoods in $G^{\prime}$ of vertices belonging to $W$.

Let $N$ denote the number of vertices in $G^{\prime}$. Since $G$ is bipartite and we do not add any cycle to it, we have that $G^{\prime}$ is also bipartite. One may easily verify that the number of vertices of $G^{\prime}$ is bounded above 
(a) Gadget associated with each $v \in$ $W \backslash\left\{w_{T}, w_{F}\right\}$.

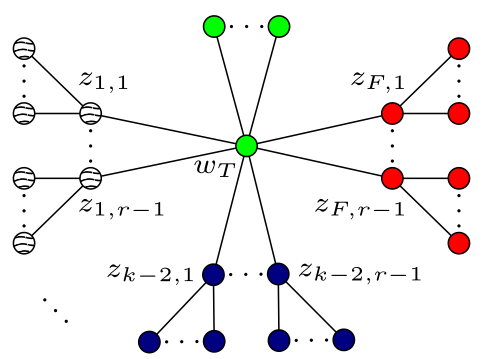

(b) Gadget associated with $w_{T}$.

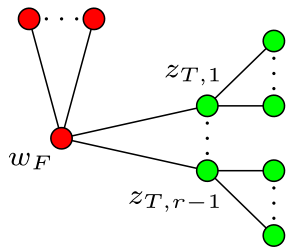

(c) Gadget associated with $w_{F}$.

Figure 4.2: Gadgets used in the proof of Theorem 4.3.

by

$$
N \leq n+n(2 \cdot n f(n)+r)+k(r-1)(1+2 \cdot n f(n)+r)
$$

Since $n>k$ and $n>r$, we obtain $N \leq 2 \cdot n^{3} f(n)+n^{3}$. Since $f(n)$ is positive and $n \geq 3$, we have

$$
N \leq 3 \cdot n^{3} f(n) \leq n^{4} f(n)=n^{\ell+4} .
$$

Consequently, the size of $G^{\prime}$ is polynomial in $n$. Moreover, observe that $N^{\frac{\ell}{\ell+4}} \leq n^{\ell}=f(n)$. Thus, we obtain $N^{1-\varepsilon} \leq N^{\frac{\ell}{\ell+4}} \leq f(n)$.

We now define a $k$-coloring $C^{\prime}: V\left(G^{\prime}\right) \rightarrow\left\{\emptyset, T, F, c_{1}, \ldots, c_{k-2}\right\}$ that extends $C$ as follows:

- $C^{\prime}(v):=C(u)$ for all $v \in S(u)$, where $u \in W$,

- $C^{\prime}\left(v_{T}\right):=T, C^{\prime}\left(v_{F}\right):=F$ for all $v \in V(G) \backslash W$,

- $C^{\prime}(v)=C^{\prime}\left(z_{i, j}\right):=c_{i}$ for all $v \in S\left(z_{i, j}\right), i \in[k-2]$ and $j \in[r-1]$,

- $C^{\prime}(v)=C^{\prime}\left(z_{T, j}\right):=T$ for all $v \in S\left(z_{T, j}\right)$ and $j \in[r-1]$,

- $C^{\prime}(v)=C^{\prime}\left(z_{F, j}\right):=F$ for all $v \in S\left(z_{F, j}\right)$ and $j \in[r-1]$.

Clearly, we have opt $\left(I^{\prime}\right)>0$ due to the colored vertices $v_{F}$ and $v_{T}$ adjacent to each uncolored vertex $v \in$ $V(G)$.

Now, let us apply algorithm $\mathcal{A}$ on instance $I^{\prime}=\left(G^{\prime}, C^{\prime}\right)$. Let $\mathcal{A}\left(I^{\prime}\right)$ be the solution obtained by algorithm $\mathcal{A}$ for instance $I^{\prime}$ and let $\operatorname{val}\left(\mathcal{A}\left(I^{\prime}\right)\right)$ be the value of this solution. Since the size of $I^{\prime}$ is polynomial in the size of $I$, the $r$-convex coloring $\mathcal{A}\left(I^{\prime}\right)$ of $G^{\prime}$ is computed in polynomial-time in the size of $I$.

Let $W^{\prime}$ be a subset of vertices in $G^{\prime}$ colored by $C^{\prime}$ such that

$$
W^{\prime}=W \cup\left\{z_{i, j}: i \in[k-2] \text { and } j \in[r-1]\right\} \cup\left\{z_{T, j}: j \in[r-1]\right\} \cup\left\{z_{F, j}: j \in[r-1]\right\} \text {. }
$$

Consider a vertex $v \in W^{\prime}$. If the color of $v$ is changed by the coloring $\mathcal{A}\left(I^{\prime}\right)$, then this coloring also recolors at least $2 \cdot n f(n)$ vertices belonging to $S(v)$ since $\mathcal{A}\left(I^{\prime}\right)$ is an $r$-convex recoloring and $|S(v)|=2 \cdot n f(n)+r$. Moreover, if the color of some vertex $u \in S(v)$ is modified, then $\mathcal{A}\left(I^{\prime}\right)$ must recolor a vertex in $W^{\prime}$ since this recoloring is $r$-convex and each color appears at least once in $(G, C)$. In both cases, we have that the value of $\mathcal{A}\left(I^{\prime}\right)$ is at least $2 \cdot n f(n)$. 
Let us now consider two cases. If $\operatorname{val}\left(\mathcal{A}\left(I^{\prime}\right)\right)<2 \cdot n f(n)$, then the $r$-convex recoloring returned by $\mathcal{A}$ for $I^{\prime}$ does not change the color of any vertex in $W^{\prime} \cup \bigcup_{v \in W^{\prime}} S(v)$. Because of the construction of $G^{\prime}$, we can conclude that every color class in the coloring $\mathcal{A}\left(I^{\prime}\right)$ induces a subgraph of $G^{\prime}$ with exactly $r$ components. Therefore, coloring $\mathcal{A}\left(I^{\prime}\right)$ restricted to vertices in $V(G)$ is a 1-convex coloring of $G$ that extends $C$, and so $I$ is a YES-instance of 1 -CCC. If $\operatorname{val}\left(\mathcal{A}\left(I^{\prime}\right)\right) \geq 2 \cdot n f(n)$, then we have $2 \cdot n f(n) \leq \operatorname{val}\left(\mathcal{A}\left(I^{\prime}\right)\right) \leq N^{1-\varepsilon} \operatorname{opt}\left(I^{\prime}\right)$. Since $N^{1-\varepsilon} \leq f(n)$, we obtain $\operatorname{opt}\left(I^{\prime}\right) \geq 2 \cdot n$. Let $n^{\prime}$ be the number of uncolored vertices in $G$, that is, $n^{\prime}=|\{v \in V(G): C(v)=\emptyset\}|$. Observe that, for each uncolored vertex $v$ in $G$, there are exactly 2 vertices, namely $v_{T}$ and $v_{F}$, adjacent to $v$ in $G^{\prime}$ that are not in $G$. Since $\operatorname{opt}\left(I^{\prime}\right) \geq 2 \cdot n$ and $n^{\prime}<n$, it follows that every feasible solution for $I^{\prime}$ must change the color of at least one vertex in $W^{\prime} \cup \bigcup_{v \in W^{\prime}} S(v)$. Thus, $I$ is a NO-instance of 1-CCC.

We conclude that $I$ is a YES-instance if, and only if, $\operatorname{val}\left(\mathcal{A}\left(I^{\prime}\right)\right)<2 \cdot n f(n)$. Therefore, 1 -CCC restricted to instances with $k$-colored bipartite graphs can be solved in polynomial-time using $\mathcal{A}$. By Theorem 4.2 , 1 -CCC is an $\mathcal{N} \mathcal{P}$-complete problem on $k$-colored bipartite graphs, and hence we have a contradiction, unless $\mathcal{P}=\mathcal{N} \mathcal{P}$.

The next theorem is a result analogous to Theorem 4.3 for the weighted version of the problem. Its proof follows a strategy similar to the one presented in the previous proof.

Theorem 4.4. Let $f: \mathbb{N} \rightarrow \mathbb{Q}$ be a function of the form $f(x)=2^{\text {poly }(x)}$. For every $r$ and $k \geq 2$, there is no $f(N)$-approximation for the $r$-CR problem on $k$-colored $N$-vertex bipartite graphs, unless $\mathcal{P}=\mathcal{N} \mathcal{P}$.

Proof. To prove this theorem, we suppose the existence of a polynomial time $f(N)$-approximation algorithm $\mathcal{A}$ for $r$-CR on $k$-colored $N$-vertex bipartite graphs and prove that $r$-CCC restricted to bipartite graphs with $k$ colors can be solved in polynomial time with algorithm $\mathcal{A}$.

Let $I=(G, C)$ be an instance of $r$-CCC consisting of a bipartite graph $G$ with $n$ vertices and a $k$-coloring $C: V(G) \rightarrow\left\{\emptyset, T, F, c_{1}, \ldots, c_{k-2}\right\}$. Assume that each color appears at least once and that $n>\max \{r, k\}$.

From instance $I$, we build an instance $I^{\prime}=\left(G^{\prime}, C^{\prime}, w\right)$ of $r$-CR problem as follows. The graph $G^{\prime}$ is obtained from $G$ by adding to it, for each uncolored vertex $v \in V(G)$, two sets $S_{T}(v)$ and $S_{F}(v)$, each one containing $r$ vertices, and making every vertex in $S_{T}(v) \cup S_{F}(v)$ adjacent to $v$. We shall use these additional vertices to eliminate feasible solutions of value 0 for $I^{\prime}$. Since $G$ is bipartite, we have that $G^{\prime}$ is also bipartite. Let $N$ denote the number of vertices of $G^{\prime}$.

The coloring $C^{\prime}$ of $G^{\prime}$ is a $k$-coloring that extends $C$ in the following way. For each uncolored vertex $v \in$ $V(G)$, we define $C^{\prime}(u)=T$ for all $u \in S_{T}(v)$, and $C^{\prime}(u)=F$ for all $u \in S_{F}(v)$. For each $v \in V(G)$, we define $C^{\prime}(v)=C(v)$.

The weight function $w: V\left(G^{\prime}\right) \rightarrow \mathbb{Q}$ is defined as follows. For each uncolored vertex $v \in V(G)$, we define $w(v)=0$ and $w(u)=1$ for every $u \in S_{T}(v) \cup S_{F}(v)$. Moreover, we define $w(v)=2 \cdot N^{2} f(N)$ for each colored vertex $v \in V(G)$, that is, for each vertex $v$ of $G$ such that $C(v) \neq \emptyset$. Observe that in the coloring $C^{\prime}$ of $G^{\prime}$, for each uncolored vertex $v \in V(G)$, the vertices in $S_{T}(v)$ and $S_{F}(v)$ have color $T$ and $F$, respectively. Consequently, every feasible solution for $I^{\prime}$ must recolor at least one vertex in $S_{T}(v) \cup S_{F}(v)$, for some uncolored vertex $v \in V(G)$. Thus, we have opt $\left(I^{\prime}\right)>0$.

Now, let us apply algorithm $\mathcal{A}$ on instance $I^{\prime}=\left(G^{\prime}, C^{\prime}, w\right)$. Let $\mathcal{A}\left(I^{\prime}\right)$ be the solution obtained by algorithm $\mathcal{A}$ for $I^{\prime}$ and let $\operatorname{val}\left(\mathcal{A}\left(I^{\prime}\right)\right)$ be the value of this solution. Note that the binary representation of the weights assigned by function $w$ has size $\mathcal{O}\left(N \log \left(N^{2} f(N)\right)\right)$, and so the size of $I^{\prime}$ is polynomial in the size of instance $I$. Thus, the $r$-convex coloring $\mathcal{A}\left(I^{\prime}\right)$ of $G^{\prime}$ is computed in polynomial time in the size of $I$. 
Consider now the following two cases. If $\operatorname{val}\left(\mathcal{A}\left(I^{\prime}\right)\right)<2 \cdot N^{2} f(N)$, then the solution returned by $\mathcal{A}$ does not change the color of any vertex colored by $C$ (that is, a vertex $v \in V(G)$ such that $C(v) \neq \emptyset)$. Moreover, the coloring $\mathcal{A}\left(I^{\prime}\right)$ restricted to vertices in $G$ is $r$-convex. Therefore, $I$ is a YES-instance of $r$-CCC. Let $n^{\prime}$ be the number of uncolored vertices in $G$, that is, $n^{\prime}=|\{v \in V(G): C(v)=\emptyset\}|$. Observe that there are exactly $2 \cdot n^{\prime} r$ vertices in $G^{\prime}$ of weight 1 . If $\operatorname{val}\left(\mathcal{A}\left(I^{\prime}\right)\right) \geq 2 \cdot N^{2} f(N)$, then opt $\left(I^{\prime}\right) \geq 2 \cdot N^{2}$, as $2 \cdot N^{2} f(N) \leq \operatorname{val}\left(\mathcal{A}\left(I^{\prime}\right)\right) \leq f(N) \operatorname{opt}\left(I^{\prime}\right)$. Since $r<n, n^{\prime}<n$ and $n \leq N$, we obtain that $2 \cdot n^{\prime} r<2 \cdot N^{2}$, and thus every $r$-convex recoloring of $\left(G^{\prime}, C^{\prime}\right)$ must change the color of at least one colored vertex of weight $2 \cdot N^{2} f(N)$. Consequently, $I$ is a NO-instance of the $r$-CCC problem.

We now conclude that $I$ is a YES-instance if, and only if, $\operatorname{val}\left(\mathcal{A}\left(I^{\prime}\right)\right)<2 \cdot N^{2} f(N)$. Therefore, $r$-CCC restricted to instances with $k$-colored bipartite graphs may be solved in polynomial time using $\mathcal{A}$. By Theorem 4.2, $r$-CCC is an $\mathcal{N} \mathcal{P}$-complete problem even on $k$-colored bipartite graphs, and so we have a contradiction, unless $\mathcal{P}=\mathcal{N} \mathcal{P}$.

\section{2 $\mathcal{W}[2]$-hardness of a parameterized unweighted version}

We now consider the decision version of the $r$-CR-BIN problem parameterized by the number of color changes, that is, the standard parameterization of $r$-CR-BIN.

Problem 4.5. PARAmeterized $r$-Convex ReColoring $(r$-CR-PARAm)

Instance: An instance $(G, C)$ of $r$-CR-BIN and a positive integer $p$.

PARAMETER: $p$.

QUESTION: Does there exist an $r$-convex recoloring of $(G, C)$ that recolors at most $p$ vertices?

Theorem 4.7 below implies that it is very unlikely that there exists an FPT-algorithm for solving $r$-CR-PARAM. To prove it, we show an FPT-reduction from a parameterized version of the CNF Satisfiability problem, namely Bounded CNF Satisfiability, which is known to be $\mathcal{W}[2]$-complete [32].

Problem 4.6. Bounded CNF SAtisfiability (Bounded-CNF-SAT)

InstancE: $A C N F$ formula $\mathcal{F}$ and a positive integer $p$.

PARAMETER: $p$.

QUESTION: Does there exist a satisfying truth assignment for $\mathcal{F}$ that assigns TRUE to at most $p$ variables?

Theorem 4.7. For every $r$ and $k \geq 2, r$-CR-PARAM is $\mathcal{W}[2]$-hard on $k$-colored bipartite graphs.

Proof. Consider an instance $(\mathcal{F}, p)$ of Bounded-CNF-SAT. Let $Q=\left\{Q_{1}, Q_{2}, \ldots, Q_{\ell}\right\}$ be the set of clauses and $X=\left\{x_{1}, x_{2}, \ldots, x_{t}\right\}$ the set of variables of formula $\mathcal{F}$. If $\ell+t \leq \max \{r, k\}$ then we reduce $(\mathcal{F}, p)$ to a YES-instance of $r$-CR-PARAM if, and only if, there exist a satisfying truth assignment for $\mathcal{F}$ that assigns TRUE to at most $p$ variables. Since $r$ and $k$ are constants, one may verify in constant-time whether there exists such a truth assignment for $\mathcal{F}$. Henceforth, let us assume that $\ell+t>\max \{r, k\}$.

First, we build a $k$-colored graph $(G, C)$, where $C: V(G) \rightarrow\left\{T, F, \emptyset, c_{1}, \ldots, c_{k-2}\right\}$, from instance $(\mathcal{F}, p)$ in the following way. For each $i \in[t]$, we associate with variable $x_{i}$ a copy of the gadget depicted in Figure 4.1(a). In this gadget, there are two sets of vertices $B_{i}^{T}=\left\{\beta_{i, 1}^{T}, \ldots, \beta_{i, r}^{T}\right\}$ and $B_{i}^{F}=\left\{\beta_{i, 1}^{F}, \ldots, \beta_{i, r}^{F}\right\}$, each one containing $r$ vertices. Moreover, there are two vertices $\alpha_{i}^{+}$and $\alpha_{i}^{-}$, and all possible edges between a vertex in $B_{i}^{T} \cup B_{i}^{F}$ and a vertex in $\left\{\alpha_{i}^{+}, \alpha_{i}^{-}\right\}$. The vertices in $B_{i}^{T}$ and in $B_{i}^{F}$ are colored with colors $T$ and $F$, respectively, that is, $C(v)=T$ for all $v \in B_{i}^{T}$, and $C(v)=F$ for all $v \in B_{i}^{F}$.

For each $j \in[\ell]$, we associate with clause $Q_{j}$ a set $S_{j}:=\left\{v_{j, 1}, v_{j, 2}, \ldots, v_{j, r}\right\}$ of vertices in $G$ such that $C(v)=T$ for every $v \in S_{j}$. For each $v \in S_{j}, v$ is adjacent to $\alpha_{i}^{+}$in $G$ if and only if literal $x_{i}$ belongs 
to $Q_{j}$; and $v$ is adjacent to $\alpha_{i}^{-}$if and only if literal $\neg x_{i}$ belongs to $Q_{j}$, where $i \in[t]$. Note that the vertices in $S_{j}$ have the same neighbors in $G$.

$G$ also has two vertices $\gamma^{T}$ and $\gamma^{F}$ such that $C\left(\gamma^{T}\right)=T$ and $C\left(\gamma^{F}\right)=F$. These vertices are adjacent to $\alpha_{i}^{+}$and $\alpha_{i}^{-}$, for every $i \in[t]$. Moreover, $G$ has a vertex $z_{i}$ adjacent to $\gamma^{T}$ such that $C\left(z_{i}\right)=c_{i}$, for each $i \in[k-2]$. One may easily check that the graph $G$ obtained from instance $(Q, X)$ is bipartite (see Figure 4.1) and that $(G, C)$ may be computed in polynomial time in the size of $(Q, X)$. Figure 4.1(b) shows the graph obtained from clause $\left(x_{1} \vee \neg x_{2} \vee x_{3}\right)$.

From graph $G$, we construct a graph $G^{\prime}$ as follows. For each $i \in[k-2]$, we replace $z_{i}$ in $G$ by new vertices $z_{i, 1}, z_{i, 2}, \ldots, z_{i, r}$ and make all of them adjacent to $\gamma^{T}$. For each $i \in[k-2]$ and $j \in[r]$, we add to $G$ a set $S\left(z_{i, j}\right)$ with $p+r$ vertices, and we make each vertex in $S\left(z_{i, j}\right)$ adjacent to $z_{i, j}$. For each $j \in[r-1]$, we add to $G$ two vertices $z_{T, j}$ and $z_{F, j}$, and edges $z_{F, j} \gamma^{T}$ and $z_{T, j} \gamma^{F}$. For each $j \in[r-1]$, we add to $G$ two sets $S\left(z_{T, j}\right)$ and $S\left(z_{F, j}\right)$, each one containing $p+r$ vertices, and we make each vertex in $S\left(z_{T, j}\right)$ adjacent to $z_{T, j}$ and each one in $S\left(z_{F, j}\right)$ adjacent to $z_{F, j}$. Figures $4.3(\mathrm{a})$ and $4.3(\mathrm{~b})$ show these vertices in the new neighborhoods of $\gamma^{T}$ and $\gamma^{F}$, respectively.

Let $W:=\left\{v \in V(G) \backslash\left\{z_{1}, \ldots, z_{k-2}\right\}: C(v) \neq \emptyset\right\}$. For each $v \in W$, we add to $G$ a set $S(v)$ of $p+r$ vertices, and we make each vertex in $S(v)$ adjacent to $v$. Moreover, for each vertex $\alpha_{i}^{+}$, we add a vertex $u_{i}$ and an edge $u_{i} \alpha_{i}^{+}$to the graph $G$, where $i \in[t]$ (see Figure 4.3(c)).

Since $G$ is bipartite and we do not add any cycle to it, we have that $G^{\prime}$ is also a bipartite graph.

We define a $k$-coloring $C^{\prime}: V\left(G^{\prime}\right) \rightarrow\left\{T, F, \emptyset, c_{1}, \ldots, c_{k-2}\right\}$ of $G^{\prime}$ as follows:

- $C^{\prime}(v):=C(u)$ for all $v \in S(u)$ and $u \in W$,

- $C^{\prime}(v)=C^{\prime}\left(z_{i, j}\right):=c_{i}$ for all $v \in S\left(z_{i, j}\right), i \in[k-2]$ and $j \in[r]$,

- $C^{\prime}(v)=C^{\prime}\left(z_{T, j}\right):=T$ for all $v \in S\left(z_{T, j}\right)$ and $j \in[r-1]$,

- $C^{\prime}(v)=C^{\prime}\left(z_{F, j}\right):=F$ for all $v \in S\left(z_{F, j}\right)$ and $j \in[r-1]$,

- $C^{\prime}\left(u_{i}\right):=F$ for all $i \in[t]$.

Clearly, the $k$-colored instance $\left(\left(G^{\prime}, C^{\prime}\right), p\right)$ of $r$-CR-PARAM can be computed from instance $(\mathcal{F}, p)$ by an FPT-algorithm with respect to $p$ since $r$ is a constant. An example of the described reduction is depicted in Figure 4.4 .

Let $C^{\prime \prime}$ be an $r$-convex total recoloring of $\left(G^{\prime}, C^{\prime}\right)$ and let $W^{\prime}$ be a subset of vertices in $G^{\prime}$ colored by $C^{\prime}$ such that

$$
W^{\prime}=W \cup\left\{z_{i, j}: i \in[k-2] \text { and } j \in[r]\right\} \cup\left\{z_{T, j}: j \in[r-1]\right\} \cup\left\{z_{F, j}: j \in[r-1]\right\} \text {. }
$$

Consider a vertex $v \in W^{\prime}$. If $C^{\prime \prime}$ changes the color of $v$, then it also changes the color of at least $p$ colored vertices in $S(v)$ since the recoloring is $r$-convex and $|S(v)|=p+r$. If $C^{\prime \prime}$ changes the color of a vertex in $S(v)$, then it must change the color of a vertex in $W^{\prime}$ due to the monochromatic stars adjacent to $\gamma^{T}$ and $\gamma^{F}$ in $G^{\prime}$, and due to the $r$-convexity of $C^{\prime \prime}$. Thus, if $C^{\prime \prime}$ recolors some vertex in $W^{\prime} \cup \bigcup_{v \in W^{\prime}} S(v)$, then it recolors more than $p$ vertices of $\left(G^{\prime}, C^{\prime}\right)$.

Therefore, if $C^{\prime \prime}$ recolors at most $p$ vertices of $\left(G^{\prime}, C^{\prime}\right)$, then it only recolors vertices belonging to $\left\{u_{1}, u_{2}, \ldots, u_{t}\right\}$. Consequently, the coloring $C^{\prime \prime}$ restricted to the vertices in $G-\left\{z_{1}, \ldots, z_{k-2}\right\}$ is 1 convex and it only uses colors $T$ and $F$. In addition, one may easily verify that $C^{\prime \prime}\left(\alpha_{i}^{+}\right)=T$ if, and only if, $C^{\prime \prime}\left(u_{i}\right)=T$. 


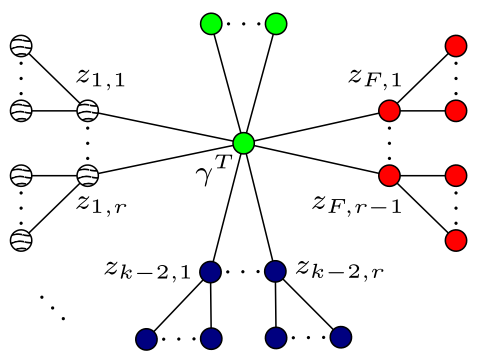

(a) Gadget associated with $\gamma^{T}$.

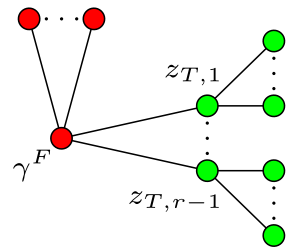

(b) Gadget associated with $\gamma^{F}$.

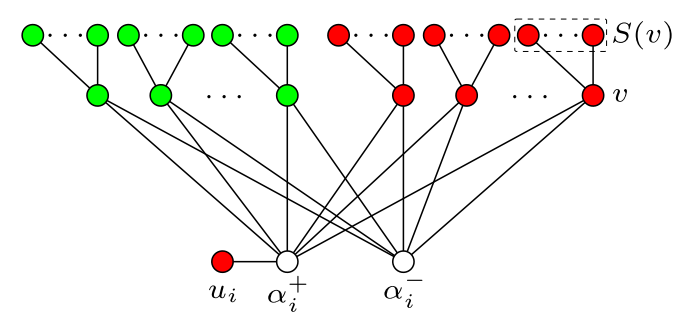

(c) Gadget associated with variable $x_{i}$.

Figure 4.3: Gadgets used in the proof of Theorem 4.7.

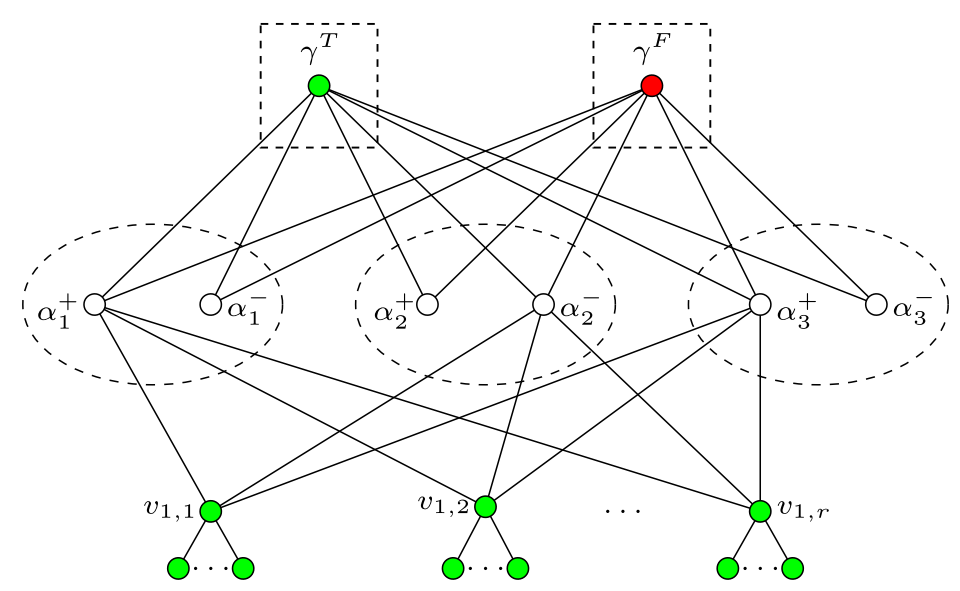

(a) The dashed ellipse represents the corresponding variable gadget. The dashed squares represent the gadgets associate with $\gamma^{T}$ and $\gamma^{F}$.

Figure 4.4: Graph obtained from the formula $\left(x_{1} \vee \neg x_{2} \vee x_{3}\right)$.

Using these remarks, we next show that $\mathcal{F}$ has a satisfying truth assignment that assigns TRUE to at most $p$ variables if, and only if, there exists an $r$-convex recoloring of $\left(G^{\prime}, C^{\prime}\right)$ that recolors at most $p$ vertices.

If $\left(G^{\prime}, C^{\prime}\right)$ is a YES-instance of the $r$-CR-PARAM problem, then there exists an $r$-convex total coloring $C^{\prime \prime}$ that recolors at most $p$ vertices in the colored graph $\left(G^{\prime}, C^{\prime}\right)$. Hence, it only recolors vertices belonging to $\left\{u_{1}, u_{2}, \ldots, u_{t}\right\}$. Since $C^{\prime \prime}$ is $r$-convex, for each $i \in[t]$, it must assign different colors among $\{T, F\}$ to the vertices $\alpha_{i}^{+}$and $\alpha_{i}^{-}$. Otherwise, either the vertices $\beta_{i, 1}^{T}, \ldots, \beta_{i, r}^{T}, \gamma^{T}$ or $\beta_{i, 1}^{F}, \ldots, \beta_{i, r}^{F}, \gamma^{F}$ would belong to $r+1$ different components of the graph induced by the corresponding color class, a contradiction to the $r$-convexity of $C^{\prime \prime}$.

Let $\Gamma: X \rightarrow\{$ TRUE, FALSE $\}$ be an assignment defined as follows. For each $i \in[t]$, we define $\Gamma\left(x_{i}\right)=$ 
TRUE if $C^{\prime \prime}\left(\alpha_{i}^{+}\right)=T$; and $\Gamma\left(x_{i}\right)=$ FALSE, otherwise. Since $C^{\prime \prime}$ is $r$-convex, we have that, for each $j \in[\ell]$, all vertices in $S_{j}$ are adjacent to a vertex $\alpha_{i}^{*}$ which has color $T$, where $i \in[t]$ and either $\alpha_{i}^{*}=\alpha_{i}^{+}$ or $\alpha_{i}^{*}=\alpha_{i}^{-}$. Thus, the assignment $\Gamma$ satisfies every clause in $Q$. Moreover, it assigns TRUE to at most $p$ variables since $C^{\prime \prime}$ recolors at most $p$ vertices in $\left\{u_{1}, u_{2}, \ldots, u_{t}\right\}$. Therefore, $(\mathcal{F}, p)$ is a YES-instance of BoundeD-CNF-SAT.

Suppose now that $(\mathcal{F}, p)$ is a YES-instance of BoundED-CNF-SAT and consider a truth assignment $\Gamma$ : $X \rightarrow\{$ TRUE, FALSE $\}$ of $\mathcal{F}$ that assigns true to at most $p$ variables. We define a total coloring $C^{\prime \prime}$ : $V\left(G^{\prime}\right) \rightarrow\left\{T, F, c_{1}, \ldots, c_{k-2}\right\}$ of $G^{\prime}$ in the following way. For each $i \in[t]$, we set $C^{\prime \prime}\left(\alpha_{i}^{+}\right)=T, C^{\prime \prime}\left(\alpha_{i}^{-}\right)=F$, and $C^{\prime \prime}\left(u_{i}\right)=T$ if $\Gamma\left(x_{i}\right)=$ TRUE; and we set $C^{\prime \prime}\left(\alpha_{i}^{+}\right)=F$ and $C^{\prime \prime}\left(\alpha_{i}^{-}\right)=T$, otherwise.

Since $\Gamma$ satisfies every clause in $Q$, we have that, for each $j \in[\ell]$, clause $Q_{j}$ contains a (positive or negative) literal of a variable $x_{i}$ which makes $Q_{j}$ true. Thus, in coloring $C^{\prime \prime}$, the vertices in $S_{j}$ belong to the connected component that contains $\gamma^{T}$ in the subgraph induced by the color class $T$. Moreover, one may easily check that, for each $i \in[t]$, the vertices $\beta_{i, 1}^{T}, \beta_{i, 2}^{T}, \ldots, \beta_{i, r}^{T}$ also belong to the connected component that contains $\gamma^{T}$ in the subgraph induced by the color class $T$. Hence, the subgraph $G^{\prime}\left[C^{\prime \prime-1}(T)\right]$ has $r$ components. In addition, note that, for each $i \in[t]$, we have that $\beta_{i, 1}^{F}, \beta_{i, 2}^{F}, \ldots, \beta_{i, r}^{F}, \alpha_{i}^{*}, \gamma^{F}$ induce a monochromatic star of color $F$ centered at $\alpha_{i}^{*}$, where $\alpha_{i}^{*}=\alpha_{i}^{+}$if $\Gamma\left(x_{i}\right)=$ FALSE, and $\alpha_{i}^{*}=\alpha_{i}^{-}$, otherwise. Thus, the subgraph $G^{\prime}\left[C^{\prime \prime-1}(F)\right]$ has $r$ components. Clearly, the color class $c_{i}$ induces a subgraph of $G^{\prime}$ with $r$ components, for every $i \in[k-2]$. Thus, the total coloring $C^{\prime \prime}$ is $r$-convex. Additionally, $C^{\prime \prime}$ modifies the colors of at most $p$ vertices of $\left(G^{\prime}, C^{\prime}\right)$ since $\Gamma$ assigns tRuE to at most $p$ variables. Therefore, $\left(G^{\prime}, C^{\prime}\right)$ is a YES-instance of $r$-CR-PARAM. 


\section{Chapter 5}

\section{Column generation approach}

In this chapter, we consider the $\mathrm{CR}$ problem (that is, $r$-CR with $r=1$ ). We denote by $(G, C, w)$ an instance of $\mathrm{CR}$ consisting of a connected graph $G$, a partial coloring $C: V \rightarrow \mathcal{C} \cup\{\emptyset\}$ and a weight function $w: V \rightarrow \mathbb{Q}_{\geq}$. As mentioned before, we assume that the weight of any uncolored vertex is 0 . Let $n:=|V(G)| \geq 3$ and $k:=|\mathcal{C}| \geq 2$.

Note that minimizing the sum of the weights of recolored vertices is equivalent to maximizing the sum of the weights of vertices that keep their initial colors. More formally, let $C^{*}$ be an optimal solution to instance $(G, C, w)$ of CR, let $R\left(C^{*}\right)=\left\{v \in V(G): C(v) \neq \emptyset\right.$ and $\left.C(v) \neq C^{*}(v)\right\}$ be the the set of vertices recolored by $C^{*}$ and let $V_{\emptyset}=\{v \in V(G): C(v)=\emptyset\}$. One may easily verify that

$$
\sum_{v \in R\left(C^{*}\right)} w(v)=\sum_{v \in V(G) \backslash V_{\emptyset}} w(v)-\sum_{v \in V(G) \backslash\left(R\left(C^{*}\right) \cup V_{\emptyset}\right)} w(v) .
$$

This remark is important since we shall define our integer linear programming (ILP) formulation for CR as a maximization problem.

\subsection{Formulation for general graphs}

In this section, we propose an integer linear programming (ILP) formulation for the convex (1-convex) recoloring problem on general graphs. Before presenting our formulation, let us introduce some notation. For each $v \in V(G)$ and $c \in \mathcal{C}$, we define a constant $w_{v, c}$ which is $w(v)$ if $C(v)=c$, and 0 otherwise. Let $\mathcal{T}(G)$ be the set of all subsets of $V(G)$ which induce connected subgraphs of $G$ and, for each $v \in V(G)$, let $\mathcal{T}_{v}(G)=\{T \in \mathcal{T}(G): v \in T\}$. For each $T \in \mathcal{T}(G)$ and $c \in \mathcal{C}$, we say that $T$ is $c$-monochromatic if color $c$ is assigned to all vertices in $T$, and we define $w_{T, c}=\sum_{v \in T} w_{v, c}$ as the weight of making $T$ $c$-monochromatic, that is, the total weight given by $T$ if we assign color $c$ to all vertices in it.

We define, for each $T \in \mathcal{T}(G)$ and $c \in \mathcal{C}$, a binary variable $x_{T, c}$ with the following meaning: $x_{T, c}=1$ if $T$ is $c$-monochromatic. 
(FMW)

$$
\begin{aligned}
& \max \sum_{T \in \mathcal{T}(G)} \sum_{c \in \mathcal{C}} w_{T, c} x_{T, c} \\
& \text { s.t. } \sum_{T \in \mathcal{T}_{v}(G)} \sum_{c \in \mathcal{C}} x_{T, c} \leq 1, \quad \text { for all } v \in V(G) \\
& \sum_{T \in \mathcal{T}(G)} x_{T, c} \leq 1, \quad \text { for all } c \in \mathcal{C} \\
& x_{T, c} \in\{0,1\}, \quad \text { for all } T \in \mathcal{T}(G), c \in \mathcal{C}
\end{aligned}
$$

Inequalities (5.1) require that each vertex receives at most one color, while inequalities (5.2) require that each color is assigned to at most one connected subgraph. We remark that a similar formulation was proposed in [71] to model a problem called Connected Assignment in Arrays, which is closely related to the convex recoloring problem restricted to paths.

We next show that every vector that satisfies inequalities (5.1)-(5.3) corresponds to a convex coloring of $G$.

Given a convex (partial) coloring $C: V(G) \rightarrow \mathcal{C}$, we define the vector $X(C) \in\{0,1\}^{|\mathcal{T}(G)| k}$ as follows. For every $T \in \mathcal{T}(G)$ and $c \in \mathcal{C}$, we have

$$
X(C)_{T, c}=\left\{\begin{array}{rr}
1 & \text { if } T=G\left[C^{-1}(c)\right], \\
0 & \text { otherwise. }
\end{array}\right.
$$

Furthermore, we define $P=\left\{x \in \mathbb{R}^{|\mathcal{T}(G)| k}: x\right.$ satisfies $(5.1),(5.2)$ and (5.3) $\}$.

Proposition 5.1. $P=\left\{X(C) \in\{0,1\}^{|\mathcal{T}(G)| k}: C\right.$ is a convex coloring of $\left.G\right\}$.

Proof. Let $C$ be a convex coloring of $G$. One may easily check that $X(C)$ satisfies inequalities $(5.1),(5.2)$ and (5.3). Therefore, we conclude that $X(C)$ belongs to $P$.

Consider now a vector $x \in P$ and let $\mathcal{T}^{\prime}=\left\{T \in \mathcal{T}(G): x_{T, c}=1\right.$ for some $\left.c \in \mathcal{C}\right\}$. We define $C: V(G) \rightarrow \mathcal{C}$ in the following way. For every $T \in \mathcal{T}(G)$ and $c \in \mathcal{C}$ such that $x_{T, c}=1$, we set $C(v)=c$ for all $v \in T$. For all $v \in V(G) \backslash\left(\bigcup_{T \in \mathcal{T}}, T\right)$, we define $C(v)=\emptyset$.

Since $x$ satisfies inequalities (5.1), it follows that $x_{T, c}+x_{T^{\prime}, d} \leq 1$ for all $T, T^{\prime} \in \mathcal{T}(G)$ such that $T \cap T^{\prime} \neq \emptyset$, and for all colors $c, d \in \mathcal{C}$. Hence, the elements of $\mathcal{T}^{\prime}$ are pairwise disjoint. Because $x$ satisfies inequalities (5.2), every color in $\mathcal{C}$ is associated with at most one element of $\mathcal{T}^{\prime}$. Using these remarks and by the fact that every set $T \in \mathcal{T}^{\prime}$ induces a connected subgraph of $G$, we conclude that $C$ is a convex coloring of $G$.

The proposed formulation has an exponential number of variables and, consequently, it is intended to be embedded in a column generation procedure. In what follows, we propose a column generation approach for solving the CR problem when the input graph is a tree.

In Section 5.2, we design an efficient algorithm to generate columns for our formulation. In Section 5.3, we prove that our formulation is at least as strong as the one proposed by Chopra et al. in [26]. Finally, in Section 5.4, we present preliminary computational experiments using our column generation algorithm on trees 


\subsection{Solving the pricing problem on trees}

In what follows, we denote by $(G, C, w)$ an instance of $\mathrm{CR}$ such that the input graph $G$ is a tree. Recall that $n=|V(G)|$ and $k=|\mathcal{C}|$.

We next show how to find an optimal solution to the linear relaxation of (FMW) on the input instance $(G, C, w)$. Initially, only a few variables are added $\left(x_{V(G), c}\right.$, for each $c \in \mathcal{C}$, for example) and the corresponding LP is solved. Then, at each iteration, we search for a new variable which has negative reduced price by solving the associated pricing problem. If such a variable is found, we add it to the so-called restricted master problem (RMP). When no variable with negative reduced price is found, the procedure halts with a certificate that the solution is optimal to the master problem; consequently, we do not need to add any of the missing variables.

We now state the pricing problem associated with the formulation proposed at the beginning of this chapter and then introduce a dynamic programming algorithm to solve it on trees. Consider the linear relaxation of formulation (FMW). Its dual can be written as follows, where $\alpha \in \mathbb{R}^{n}$ and $\beta \in \mathbb{R}^{k}$ are the vectors of dual variables associated with inequalities (5.1) and (5.2), respectively.

$$
\begin{aligned}
\min & \sum_{v \in V(G)} \alpha_{v}+\sum_{c \in \mathcal{C}} \beta_{c} \\
\text { s.t. } & \sum_{v \in T} \alpha_{v}+\beta_{c} \geq w_{T, c}, \\
& \alpha, \beta \geq \mathbf{0}
\end{aligned}
$$

Therefore, given $(\alpha, \beta) \in \mathbb{R}^{n} \times \mathbb{R}^{k}$, the corresponding pricing problem is to find a pair $\left(T^{*}, c^{*}\right)$, such that

$$
\left(T^{*}, c^{*}\right) \in \arg \min _{(T, c) \in \mathcal{T}(G) \times \mathcal{C}} \sum_{v \in T} \alpha_{v}+\beta_{c}-w_{T, c} .
$$

We now introduce a dynamic programming algorithm to solve this pricing problem. For ease of presentation, we show a simplified version of the algorithm in which the value of the optimal solution is kept, while the solution itself is discarded. The complete version of the algorithm may be easily deduced from this simplified version. Considering this observation and rewriting (5.6), we are interested in solving the following problem:

$$
\eta=\min _{(T, c) \in \mathcal{T}(G) \times \mathfrak{C}} \sum_{v \in T}\left(\alpha_{v}-w_{v, c}\right)+\beta_{c} .
$$

Before presenting the algorithm, we discuss the basic ideas behind it. Firstly, we decompose the problem into $k$ subproblems, called color-subproblems. Each color-subproblem consists in finding a $T \in$ $\mathcal{T}(G)$ which minimizes $\sum_{v \in T}\left(\alpha_{v}-w_{v, c}\right)+\beta_{c}$, for a fixed color $c$. Hence, given a fixed color $c \in \mathcal{C}$, in the corresponding color-subproblem we seek a subtree of $G$ with minimum total cost, where the cost of a vertex $v$ is $\alpha_{v}-w_{v, c}$. Each color-subproblem is decomposed into $n$ subproblems, which are called vertex-subproblems. In each vertex-subproblem, we are interested in finding a minimum total cost subtree of $G$ which contains a given vertex. We solve this subproblem for each vertex $v \in V(G)$ and take a minimum total cost subtree among them. Thus, we obtain an optimal solution for the color-subproblem corresponding to a given fixed color.

We split the algorithm into two subroutines, namely SOLVE-PRICING-BY-DP (see Algorithm 1) and MIN-RED-PRICE-SUBTREE (see Algorithm 2). We omit the pseudocode of the subroutine BUild-Rooted-tree $(G, v)$, which simply calculates a vector $p$, such that, for each $u \in V(G), p_{u}$ is 
the parent of $u$ when the tree $G$ is rooted at $v$ (for simplicity, we define $p_{v}=v$ ).
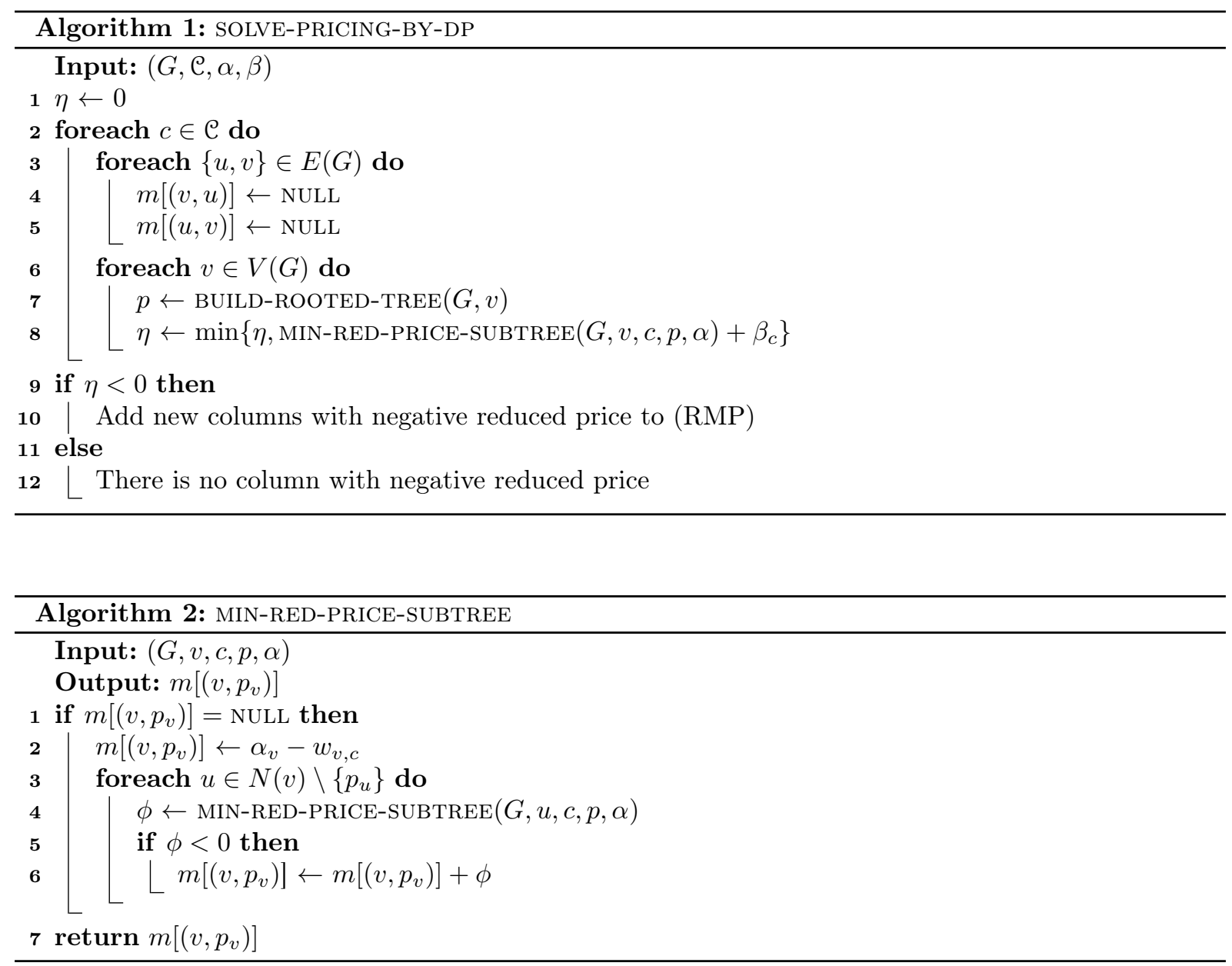

For every vertex $v \in V(G)$ and color $c \in \mathcal{C}$, we define $\operatorname{vAL}_{c}(G, v)$ as the following recurrence

$$
\operatorname{VAL}_{c}(G, v)=\alpha_{v}-w_{v, c}+\sum_{u \in N(v)} \min \left\{0, \operatorname{vAL}_{c}\left(G_{u v}, u\right)\right\},
$$

where $N(v)$ is the set of neighbors of $v$ in $G$, and $G_{u v}$ is the subtree of $G$ - uv (the graph obtained from $G$ by removing edge $u v$ ) which contains vertex $u$. One may easily check that the value $\operatorname{vAL}_{c}(G, v)$ is computed by algorithm MIN-RED-PRICE-SUBTREE. Note that the vertex-subproblems have the so-called optimal substructure. Thus, by induction on the number of vertices in $G$, one may prove that $\operatorname{vaL}_{c}(G, v)$ is equal to the value of an optimal solution of the corresponding vertex-subproblem. In what follows, we formally prove this claim.

For each color $c \in \mathcal{C}$, we define $\eta_{c}=\min _{T \in \mathcal{T}(G)} \sum_{v \in T}\left(\alpha_{v}-w_{v, c}\right)+\beta_{c}$. Clearly, we can subdivide the pricing problem into $k$ subproblems so that $\eta=\min _{c \in \mathcal{C}} \eta_{c}$. Thus, for a fixed color $c \in \mathcal{C}$, we are interested in finding

$$
\min _{T \in \mathcal{T}(G)} \sum_{v \in T}\left(\alpha_{v}-w_{v, c}\right) .
$$

To prove that algorithm SOLVE-PRICING-BY-DP correctly solves the pricing problem, it suffices to prove the following proposition. 
Proposition 5.2. For every $v \in V(G)$ and $c \in \mathcal{C}$, we have

$$
\operatorname{VAL}_{c}(G, v)=\min _{T \in \mathcal{T}(G): v \in T} \sum_{u \in T}\left(\alpha_{u}-w_{u, c}\right)
$$

Proof. We prove the claimed result by induction on $n$, the number of vertices in the tree $G$. If $n=1$, then the result follows trivially.

Suppose now that, for every tree $H$ such that $|V(H)|<n$ and every $z \in V(H)$, we have

$$
\operatorname{VAL}_{c}(H, z)=\min _{T \in \mathcal{T}(H): z \in T} \sum_{u \in T}\left(\alpha_{u}-w_{u, c}\right)
$$

Let $T^{*} \in \arg \min _{T \in \mathcal{T}(G): v \in T} \sum_{u \in T}\left(\alpha_{u}-w_{u, c}\right)$. Thus, for each $z \in N(v)$, we have

$$
\sum_{u \in T^{*} \cap V\left(G_{z v}\right)}\left(\alpha_{u}-w_{u, c}\right) \geq \min _{T \in \mathcal{T}\left(G_{z v}\right): z \in T} \sum_{u \in T}\left(\alpha_{u}-w_{u, c}\right)=\operatorname{VAL}_{c}\left(G_{z v}, z\right),
$$

where the last equality is due to the induction hypothesis. Observe now that

$$
\sum_{u \in T^{*}}\left(\alpha_{u}-w_{u, c}\right) \geq \alpha_{v}-w_{v, c}+\sum_{z \in N(v)} \operatorname{VAL}_{c}\left(G_{z v}, z\right)
$$

Therefore, we obtain that $\sum_{u \in T^{*}}\left(\alpha_{u}-w_{u, c}\right) \geq \operatorname{vAL}_{c}(G, v)$ and, consequently,

$$
\operatorname{VAL}_{c}(G, v)=\min _{T \in \mathcal{T}(G)} \sum_{u \in T}\left(\alpha_{u}-w_{u, c}\right)
$$

Note that, when computing $\operatorname{VAL}_{c}(G, v)$ for every $v \in V(G)$, many recursive calls have the same parameters. To save computation time, we store the solutions of the subproblems in a memoization table instead of recalculating them several times. Using Proposition 5.2, one may easily verify the following proposition.

Proposition 5.3. The pricing problem can be solved in time $\mathcal{O}\left(k n^{2}\right)$ on n-vertex $k$-colored trees.

To conclude our discussion about the pricing problem, we mention the Connected $\ell$-Subgraph problem which is defined as follows. Given a vertex-weighted graph, in this problem the objective is to find a connected subgraph with $\ell$ vertices such that the sum of the weights of its vertices is minimized. Hochbaum and Pathria [43] presented an algorithm that computes in time $\mathcal{O}\left(\ell^{2} n\right)$ an optimal solution to the Connected $\ell$-Subgraph problem on $n$-vertex trees.

We remark that our pricing problem with a fixed color (that is, the color-subproblem) can be solved in $\mathcal{O}\left(n^{3}\right)$ time using Hochbaum and Pathria's algorithm. However, it is easy to see that we may optimally solve the color-subproblem in time $\mathcal{O}\left(n^{2}\right)$ using the algorithm MIN-RED-PRICE-SUBTREE with a fixed color.

\subsection{Comparing formulations for trees}

We recall that $(G, C, w)$ denotes an instance of $\mathrm{CR}$ where $G$ is a tree, $C: V \rightarrow \mathcal{C} \cup\{\emptyset\}$ is a partial coloring of $G$ and $w: V \rightarrow \mathbb{Q} \geq$ is a weight function. Let $n=|V(G)|, m=|E(G)|$ and $k=|\mathcal{C}|$. 
We next present the integer linear programming (ILP) formulation introduced by Chopra et al. in [26] for modeling the $\mathrm{CR}$ problem on trees. In this formulation, for each $v \in V(G)$ and $c \in \mathcal{C}$, there is a binary variable $y_{v, c}$ with the following meaning: vertex $v$ has color $c$ if and only if $y_{v, c}=1$. For each $u v \in E(G)$ and $c \in \mathcal{C}$, there is a binary variable $z_{u v, c}$ such that $z_{u v, c}=1$ if and only if vertices $u$ and $v$ have color $c$. For simplicity, we say that edge $u v$ "receives" color $c$ if $z_{u v, c}=1$.

(CRLS)

$$
\begin{aligned}
& \max \sum_{v \in V(G)} \sum_{c \in \mathcal{C}} w_{v, c} y_{v, c} \\
& \text { s.t. } \sum_{c \in \mathcal{C}} y_{v, c} \leq 1, \quad \text { for all } v \in V(G) \\
& \sum_{v \in V(G)} y_{v, c}-\sum_{u v \in E(G)} z_{u v, c} \leq 1, \quad \text { for all } c \in \mathcal{C} \\
& z_{u v, c}-y_{v, c} \leq 0 \\
& z_{u v, c}-y_{u, c} \leq 0 \\
& y_{v, c} \in\{0,1\} \text {, } \\
& \text { for all } u v \in E(G), c \in \mathcal{C} \\
& z_{u v, c} \in\{0,1\} \text {, } \\
& \text { for all } v \in V(G), c \in \mathcal{C} \\
& \text { for all } u v \in E(G), c \in \mathcal{C}
\end{aligned}
$$

Inequalities (5.8) require that each vertex receives at most one color. Inequalities (5.9) require that the number of edges receiving a given color is at least the number of vertices receiving the same color minus one. Inequalities (5.10) require that, if an edge $u v$ receives a color $c$, then its endpoints $u$ and $v$ must have color $c$ as well (that is, these inequalities are "linking" variables $z$ to $y$ ). Inequalities (5.9) and (5.10) together force that $|E(H)|=|V(H)|-1$, for each subgraph $H$ induced by the vertices receiving a common color. This implies that $H$ is a tree and, thus, it is connected. Note that, if we replace the integrality constraints (5.12) by non-negative constraints, the $z$ variables will still have integer values in the optimal solution.

The objective function maximizes over the total coloring weight. In both ILP formulations presented here, as well as in the one introduced in [19], the maximization version of the problem is considered.

We denote by (CRLS) and (FMW) the ILP formulations presented in this section and at the beginning of Chapter 5, respectively. In what follows, we show that formulation (FMW) (specialized for the case of trees) is at least as strong as (CRLS).

Recall that $\mathcal{T}$ is the set of all subsets of $V(G)$ that induce subtrees of $G$, and let $q$ be the size of $\mathcal{T}$. Given $T \in \mathcal{T}$, let $E(T)$ be the set of edges of the subtree induced by $T$. For each $u v \in E(G)$, let $\mathcal{T}(u v)=\{T \in \mathcal{T}: u v \in E(T)\}$. Let $\mathcal{P}$ be the polytope associated with the linear relaxation of formulation (FMW) with input instance $(G, C, w)$, and let $\mathcal{Q}$ be the polytope associated with the linear relaxation of formulation (CRLS) with the same input instance.

We next define a function $f: \mathbb{R}^{k q} \rightarrow \mathbb{R}^{k n} \times \mathbb{R}^{k m}$ for mapping the vectors of $\mathcal{P}$ onto the same vector space in which $\mathcal{Q}$ is contained. Given $x \in \mathcal{P}$, we define $f(x)=(y, z)$, where $y \in \mathbb{R}^{k n}$ and $z \in \mathbb{R}^{k m}$, in the following way:

$$
\begin{aligned}
y_{v, c} & =\sum_{T \in \mathcal{T}(v)} x_{T, c}, \text { for each } v \in V(G) \text { and } c \in \mathcal{C} \\
z_{u v, c} & =\sum_{T \in \mathcal{T}(u v)} x_{T, c}, \text { for each } u v \in E(G) \text { and } c \in \mathcal{C}
\end{aligned}
$$

Let $\mathcal{P}_{\mathrm{MAP}}=f(\mathcal{P})$ be the polyhedron obtained by mapping $\mathcal{P}$ into the space $\mathbb{R}^{k n} \times \mathbb{R}^{k m}$, that is, 
$\mathcal{P}_{\mathrm{MAP}}=\left\{(y, z) \in \mathbb{R}^{k n} \times \mathbb{R}^{k m}: x \in \mathcal{P}\right.$ and $\left.f(x)=(y, z)\right\}$. In the remainder of this section, we show that every vector of $\mathcal{P}_{\text {MAP }}$ belongs to $\mathcal{Q}$.

Proposition 5.4. $\mathcal{P}_{\text {PROJ }} \subseteq \mathcal{Q}$.

Proof. Let $(y, x)$ be a vector belonging to $\mathcal{P}_{\mathrm{PROJ}}$ and $x \in \mathcal{P}$ be a vector such that $f(x)=(y, z)$. Let $c \in \mathcal{C}$ be a color. In what follows, we prove that $(y, z)$ satisfies inequalities $(5.8)$ and, for the fixed color $c$, it satisfies (5.9) and (5.10) of formulation (CRLS).

Claim 5.5. $(y, z)$ satisfies inequalities (5.8).

Proof. Considering the definition of $\mathcal{P}_{\text {PROJ }}$ and equalities (5.13), we have

$$
\sum_{c \in \mathcal{C}} y_{v, c}=\sum_{c \in \mathcal{C}} \sum_{T \in \mathcal{T}(v)} x_{T, c} \leq 1
$$

for every $v \in V(G)$ and, thus, the result follows easily.

Claim 5.6. $(y, z)$ satisfies inequalities (5.9).

Proof. For each $c \in \mathcal{C}$, it follows from equalities (5.13) and (5.14) that

$$
\sum_{v \in V(G)} y_{v, c}-\sum_{u v \in E(G)} z_{u v, c}=\sum_{v \in V(G)} \sum_{T \in \mathcal{T}(v)} x_{T, c}-\sum_{u v \in E(G)} \sum_{T \in \mathcal{T}(u v)} x_{T, c} .
$$

For a fixed $T^{\prime} \in \mathcal{T}$, variable $x_{T^{\prime}, c}$ appears $\left|T^{\prime}\right|$ times in first double summation and $\left|E\left(T^{\prime}\right)\right|$ times in the second double summation. Since $E\left(T^{\prime}\right)$ is the edge set of the subtree induced by $T^{\prime}$, we have $\left|E\left(T^{\prime}\right)\right|=\left|T^{\prime}\right|-1$. Consequently, we can rewrite the right side of the equation as $\sum_{T \in \mathcal{T}} x_{T, c}(|T|-|E(T)|)=$ $\sum_{T \in \mathcal{T}} x_{T, c}$, which, by inequality (5.2), is at most 1. Therefore, we conclude that inequalities (5.9) are satisfied by $(y, z)$.

Claim 5.7. $(y, z)$ satisfies inequalities (5.10).

Proof. Note that, for every $u v \in E(G)$, we have $z_{u v, c}-y_{v, c}=\sum_{T \in \mathcal{T}(u v)} x_{T, c}-\sum_{T \in \mathcal{T}(v)} x_{T, c}$.

For a fixed $T^{\prime} \in \mathcal{T}$, observe that each time $x_{T^{\prime}, c}$ appears in the first summation, it must appear in the second summation. Thus, the sum of these two terms is at most zero. Clearly, the same arguments can be used when we replace vertex $v$ by vertex $u$. Therefore, inequalities (5.10) are satisfied by $(y, z)$.

By the previous claims, we conclude that $(y, z)$ belongs to $\mathcal{Q}$. Therefore, it immediatly follows that $\mathcal{P}_{\text {PROJ }} \subseteq \mathcal{Q}$.

\subsection{Preliminary experiments on trees}

As mentioned in the introduction, some important problems in Biology that are related to phylogenetic trees can be modeled in terms of the convex recoloring problem on trees [60, 61]. Therefore, efficient solution methods for the CR problem on trees are of great interest due to its application in Bioinformatics.

In this section, we present some computational experiments of a column generation algorithm using the formulation we proposed in Section 5.1 (namely, formulation (FMW)). Additionally, we compare our results with the ones obtained by using the solution method proposed by Chopra et al. [26] which is based on formulation (CRLS). To the best of our knowledge, the algorithm described in [26] is the fastest solution method for the CR problem on trees described in the literature. 
The dataset used in our experiments is the same as in [19], which consists of instances that come from an application in the study of phylogenetic trees. Our implementations were coded in $\mathrm{C}++$ programming language, using CPLEX as the mixed integer linear programming solver. The machine configurations are the following: $65 \mathrm{~GB}$ of RAM and $1.6 \mathrm{GHz}$ processor.

The dataset consists of 10 trees and, for each of them, we generate 12 different initial (total) colorings in the same way described in [19]. Hence, altogether there are 120 different instances. We grouped the instances in two categories, according to the ratio between the number of different colors in the initial coloring and the number of vertices of the graph. One group contains 80 instances with low number of colors (i.e., ratio $\approx 5 \%$ ), and the other group, containing the instances with high number of colors (i.e., ratio $\approx 50 \%$ ), has 40 instances. In Tables 5.1 and 5.2, we show the results obtained with these two groups of instances. For a given tree in our dataset, columns $\widetilde{\mid \mathcal{E}} \mid$ of Tables 5.1 and 5.2 show the average number of colors in the instances consisting of this tree and colorings with low number of colors and colorings with high number of colors, respectively. The running times are given in seconds. Columns $n$ of Tables 5.1 and 5.2 contain the number of vertices of the trees in the corresponding instances.

\begin{tabular}{rrrrrrr}
\hline & & \multicolumn{2}{c}{ avg. run time } & & \multicolumn{2}{c}{ avg. \# of variables } \\
$n$ & $\widetilde{\mid C} \mid$ & $($ FMW $)$ & $($ CRLS $)$ & & $($ FMW $)$ & $($ CRLS $)$ \\
\hline 213 & 6 & 0.19 & 0.17 & & 21 & 2914 \\
301 & 11 & 0.44 & 0.34 & & 26 & 7061 \\
404 & 13 & 1.13 & 0.69 & & 31 & 11096 \\
567 & 16 & 2.02 & 0.56 & & 27 & 18694 \\
636 & 16 & 4.49 & 1.63 & & 43 & 20971 \\
710 & 21 & 6.03 & 2.09 & & 46 & 30331 \\
813 & 25 & 9.84 & 3.45 & & 62 & 41843 \\
981 & 30 & 26.80 & 4.99 & & 68 & 59075 \\
1441 & 39 & 56.95 & 21.33 & & 101 & 114879 \\
1577 & 43 & 79.58 & 25.16 & 111 & 135579 \\
\hline
\end{tabular}

Table 5.1: Instances with low number of colors in the initial coloring.

\begin{tabular}{rrrrrrr}
\hline & & \multicolumn{2}{c}{ avg. run time } & & \multicolumn{2}{c}{ avg. \# of variables } \\
$n$ & $\widetilde{\mid \mathcal{C}} \mid$ & $($ FMW $)$ & (CRLS) & & (FMW) & (CRLS) \\
\hline 213 & 104 & 1.07 & 4.26 & & 102 & 44200 \\
301 & 155 & 2.38 & 13.24 & & 150 & 93305 \\
404 & 202 & 6.39 & 22.90 & & 187 & 163417 \\
567 & 274 & 26.75 & 20.89 & & 221 & 310725 \\
636 & 315 & 30.76 & 63.85 & & 298 & 401318 \\
710 & 347 & 37.74 & 81.12 & & 319 & 492747 \\
813 & 411 & 65.05 & 169.53 & & 382 & 667875 \\
981 & 483 & 133.05 & 243.08 & & 460 & 947163 \\
1441 & 716 & 388.87 & 822.90 & & 690 & 2063516 \\
1577 & 803 & 505.42 & 1104.32 & & 725 & 2533435 \\
\hline
\end{tabular}

Table 5.2: Instances with high number of colors in the initial coloring.

For all instances of the dataset, both methods obtained integer solutions by solving only the respective linear relaxations. This indicates that both formulations give a good approximation for the convex hull of the integer points for this type of instance. One important remark is that the linear relaxation proposed 
in [19] did not find integer solutions to all instances of this same dataset.

As Tables 5.1 and 5.2 indicate, on instances with low number of colors compared to the number of vertices, (CRLS) is faster than (FMW). However, when the number of colors is high, (FMW) performs better than (CRLS). This may be explained by the fact that the number of added variables in formulation (FMW) is very small (less than $|V(G)| / 2$ in all cases), while the number of variables in formulation (CRLS) is $\Theta((|V(G)|+|E(G)|) \cdot|\mathcal{C}|)$. Note that in (CRLS) all variables are added a priori, while in (FMW) each variable is generated by the algorithm described in Section 5.2, which runs in $\Theta\left(|V(G)|^{2} \cdot|\mathcal{C}|\right)$ time. 


\section{Part III}

\section{On $k$-fold coloring}





\section{Chapter 6}

\section{The $k$-fold coloring problem}

This chapter introduces basic definitions and presents some results in the literature of the $k$-fold coloring problem (see Section 6.1). Additionally, in Section 6.2, we present an overview of the results obtained during our studies on this problem.

\subsection{Basic definitions and known results}

A $k$-fold $x$-coloring of a graph $G$ is an assignment of (at least) $k$ distinct colors from the set $[x]:=$ $\{1,2, \ldots, x\}$ to each vertex such that any two adjacent vertices are assigned disjoint sets of colors. The $k$-th chromatic number of $G$, denoted $\chi_{k}(G)$, is the smallest value $x$ such that $G$ admits a $k$-fold $x$-coloring [72]. Obviously, $\chi_{1}(G)=\chi(G)$ is the conventional chromatic number. Additionally, one may easily verify that $\chi_{k}(G) \leq k \cdot \chi(G)$ for all $k \in \mathbb{N}$. Observe that $\chi_{k}(G)$ is equivalently defined if each vertex is restricted to receive exactly $k$ colors.

In Figure 6.1, we show $k$-fold $x$-colorings of an odd cycle $C_{5}$ with 5 vertices. We have a 1 -fold 3 -coloring (Figure 6.1(a)) and a 2-fold 5-coloring (Figure 6.1(b)). Actually, they are optimal colorings for $k=1$ and $k=2$, respectively, as $\chi_{1}\left(C_{5}\right)=3$ and $\chi_{2}\left(C_{5}\right)=5$ [18]. In Figure 6.1(a), note that we could have removed one color from $v_{2}$ since it is assigned more than $k=1$ colors.

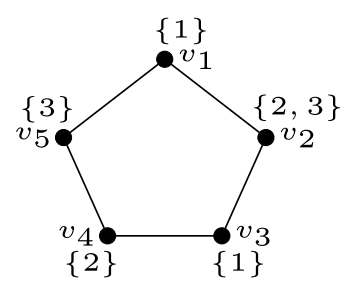

(a) 1-fold 3-coloring.

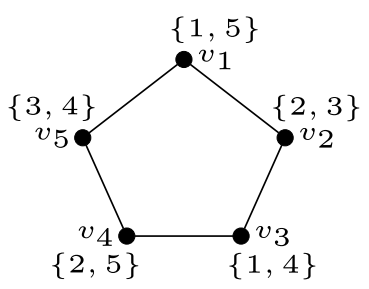

(b) 2-fold 5-coloring.

Figure 6.1: Examples of $k$-fold $x$-colorings of a cycle $C_{5}$.

The $k$-fold coloring problem is that of finding, for a given graph $G$, a coloring with $\chi_{k}(G)$ colors. There is a vast literature on the (1-fold) coloring problem, comprising several aspects of the problem such as computational complexity, combinatorial properties, polyhedral studies and solution methods. Recall that determining $\chi(G)$ is an $\mathcal{N} \mathcal{P}$-hard problem. In fact, it is $\mathcal{N} \mathcal{P}$-hard to approximate the chromatic number within $n^{1-\varepsilon}$ on $n$-vertex graphs, for every $\varepsilon>0$ [80]. As a direct consequence, for each fixed $k \in \mathbb{N}$, it is $\mathcal{N} \mathcal{P}$-hard to approximate $\chi_{k}(G)$ within $\frac{n^{1-\varepsilon}}{k}$ on $n$-vertex graphs $G$, for every $\varepsilon>0$. 
Let $G$ be a graph and let us denote by $\mathcal{S}(G)$ the set of all stable sets of $G$. The fractional chromatic number of $G$, to be denoted $\bar{\chi}(G)$, is the value of an optimal solution of the following linear programming formulation.

$$
\begin{aligned}
& \min \sum_{S \in \mathcal{S}(G)} x_{S} \\
& \text { s.t. } \sum_{S \in \mathcal{S}(G): v \in S} x_{S} \geq 1 \quad \text { for all } v \in V(G) \text {, } \\
& x_{S} \geq 0 \quad \text { for all } S \in \mathcal{S}(G) .
\end{aligned}
$$

This parameter relates to the $k$-th chromatic number as follows:

$$
\bar{\chi}(G)=\inf \left\{\frac{\chi_{k}(G)}{k}: k \in \mathbb{N}\right\} .
$$

It is known that $\omega(G) \leq \bar{\chi}(G) \leq \chi(G)$ and $\frac{|V(G)|}{\alpha(G)} \leq \bar{\chi}(G)$ [70]. As observed in [18], the following bounds hold and they are tight, for instance, for perfect graphs.

Fact 6.1. For every graph $G$ and every $k \in \mathbb{N}, \omega(G) \leq \bar{\chi}(G) \leq \frac{\chi_{k}(G)}{k} \leq \chi(G)$.

In comparison with the 1 -fold case, there are only a few studies regarding the $k$-fold coloring. From the theoretical point of view, one of the reasons for this situation could be the fact that a $k$-fold coloring of a graph $G$ can be given by a 1-fold coloring of the graph $G \circ K_{k}$, the lexicographic product of $G$ by a clique with $k$ vertices. More precisely, $\chi_{k}(G)=\chi_{1}\left(G \circ K_{k}\right)$. Recall that $G \circ K_{k}$ is obtained by replacing each vertex of $G$ by the clique $K_{k}$ and making two vertices in $G \circ K_{k}$ adjacent whenever the corresponding vertices in $G$ are adjacent.

From the computational point of view, this approach, however, may suffer from many drawbacks, mainly because $G \circ K_{k}$ is $k$ times larger than $G$ and the $k$ vertices associated with each vertex of $G$ play essentially the same role in the coloring $G \circ K_{k}$. In order to make a solution method for finding an 1-fold optimal coloring efficient to the $k$-fold case, one should have to explore the special structure of $G \circ K_{k}$ to compensate the increase in the number of vertices. Moreover, in an enumerative procedure, the many symmetric solutions arising from the multiple copies of each vertex of $G$ in $G \circ K_{k}$ may cause serious inconveniences.

As an alternative, one could try to tackle the $k$-fold coloring problem directly (on $G$ ). However, it does not seem reasonable to disregard all results about the conventional coloring. This work contributes in this direction and focuses on polyhedral studies. For studies on the $k$-fold coloring problem from the perspective of structural graph theory, the reader is referred to [18, 28, 50, 69, 72, 73].

\subsection{Contributions}

First, in Chapter 7, we propose an integer linear programming formulation for the $k$-fold coloring problem based on class representatives. Class representatives were introduced in [16, 17] to model the vertex coloring problem, and since then have been used to model several other problems that aim to cluster elements of a universe according to some property (see e.g. [8, 22, 44, 45]). For $k \geq 2$, the formulation we propose for a graph $G$ is more compact than the (1-fold) representatives formulation for the lexicographic product $G \circ K_{k}$. They coincide when $k=1$. Also in Chapter 7 , we define a polytope $\mathcal{P}_{k}(G)$ associated with this formulation and show some important basic properties of such polytope. 
In Chapters 8 and 9, we study the facial structure of the polytope $\mathcal{P}_{k}(G)$.

We profit from the known facial studies for $\mathcal{P}_{1}(G)$ in two ways. In Section 8.1, we present a lifting theorem that combines a facet of $\mathcal{P}_{k}(G)$ and a facet of $\mathcal{P}_{\ell}(G)$ into a facet of $\mathcal{P}_{k+\ell}(G)$ as well as we show specific liftings from facets of $\mathcal{P}_{k}(G)$ to facets of $\mathcal{P}_{k+1}(G)$. In Section 8.2, we show how to project facets from $\mathcal{P}_{1}\left(G \circ K_{k}\right)$ into facets of $\mathcal{P}_{k}(G)$. In both cases, we can recover to $\mathcal{P}_{k}(G)$ most of the facet-defining inequalities of the coloring polytope derived in [16]. In Section 8.3, we show how to transform to $\mathcal{P}_{k}(G)$ any facet-defining inequality of the stable set polytope associated with the subgraph of $G$ induced by the anti-neighborhood of each vertex.

Finally, in Sections 9.2 and 9.3, we derive a class of facet-defining inequalities induced by webs and antiwebs, which are structures that play an important role in the description of stable set and coloring polytopes $[24,78]$. We also characterize a criticality property required by these graphs to induce facets of $\mathcal{P}_{k}(G)$ in Section 9.1. These results extend and generalize the results in [16, 65].

The results presented in this part were obtained in collaboration with Manoel Campêlo and Marcio C. Santos, and published in [20]. 


\section{Chapter 7}

\section{Representatives polytope}

In this chapter, we present the concept of class representatives and, based on this, we propose an integer linear programming formulation for the $k$-fold coloring problem. These are the topics discussed in Section 7.1. Moreover, in Section 7.2, we define a polytope associated with this formulation and we show some important basic properties of this polytope.

\subsection{Formulation}

Each color class (vertices with the same color) defines a stable set (subset of pairwise nonadjacent vertices) of the graph. Therefore, a coloring is a partition of the vertices into stable sets. A family of stable sets can be described by the idea of class representatives [16, 17], as follows.

Henceforth, we consider a graph $G$ with $n=|V(G)|$ vertices and $m=|E(G)|$ edges. Let $\bar{G}$ be the complement of $G$ and $\bar{m}=|E(\bar{G})|$ be the number of edges of $\bar{G}$ (anti-edges of $G$ ). Given an ordering on $V(G)$, the representative of a stable set $S$ is its minimum vertex $r$ (which represents each vertex in $S$ ), and $S$ can be uniquely described by a binary vector $x^{S} \in \mathbb{B}^{n+\bar{m}}$, indexed by the $n$ vertices and $\bar{m}$ edges of $\bar{G}$, having 1 in the entries corresponding to $r \in S$ and $r v \in \bar{E}$, for each $v \in S \backslash\{r\}$. Let $\mathcal{S}$ be the set of all stable sets of $G$ and $X^{\mathcal{S}}=\left\{x^{S}: S \in \mathcal{S}\right\}$.

A family $\mathcal{F} \subseteq 2^{\mathcal{S}}$ of stable sets has a unique description by vectors from $X^{\mathcal{S}}$. Moreover, all these vectors (each of dimension $n+\bar{m}$ ) can be merged in a single binary vector $x^{\mathcal{F}}=\sum_{S \in \mathcal{S}} x^{S}$ whenever the representative vertices are all different (this occurs, for instance, if the stable sets in $\mathcal{F}$ are disjoint). Such a description is unambiguous, that is, $x^{\mathcal{F}} \neq x^{\mathcal{F}^{\prime}}$ if $\mathcal{F} \neq \mathcal{F}^{\prime}$. This idea was used in [16] to model the coloring problem. Since then it has been applied to model several other problems that aim to cluster elements from a universe $[8,22,44,45]$.

Here, we use the same notion of class representatives to model the $k$-fold coloring problem. In this context, a coloring is still seen as a family of stable sets. For $k=1$, we can always assume that these stable sets have different representatives (or even be disjoint). However, for $k>1$, sets in the family may even coincide. Now, we can admit that a vertex represents from 0 to $k$ stable sets. We need to adapt the representatives description of a family.

Firstly, let us introduce the following definitions. Let $\prec$ be an ordering on $V(G)$. Let $\bar{N}^{-}(v)=\{u \prec$ $v: u v \in E(\bar{G})\}$ and $\bar{N}^{+}(v)=\{v \prec u: u v \in E(\bar{G})\}$ be the negative and postive anti-neighborhood of $v$, respectively. Denote $\bar{N}[v]=\bar{N}^{+}(v) \cup \bar{N}^{-}(v) \cup\{v\}$ and $\bar{N}^{-}[v]=\bar{N}^{-}(v) \cup\{v\}$. For each $i \in[k], v \in V(G)$ and $u \in \bar{N}^{-}[v]$, define a binary variable $x_{u v}^{i} \in \mathbb{B}$, which receives 1 if, and only if, the $i$-th color class represented by $u$ is nonempty and includes $v$. Notice that we are assuming that each vertex $v$ represents $k$ 
(possibly empty) color classes, and $x_{v v}^{i}=1$ marks that the $i$-th color class represented by $v$ is nonempty.

The representatives formulation for 1 -fold coloring [16] can be easily generalized for $k$-fold coloring as follows.

$$
\begin{array}{rlrl}
\mathcal{R}_{k}(G) \quad \min & \sum_{i=1}^{k} \sum_{v \in V(G)} x_{v v}^{i} & \\
\text { s.t. } \sum_{i=1}^{k} \sum_{u \in \bar{N}^{-}[v]} x_{u v}^{i} \geq k & & \forall v \in V(G), \\
& x_{v u}^{i}+x_{v w}^{i} \leq x_{v v}^{i} & & \forall v \in V(G), \forall u, w \in \bar{N}^{+}(v), u w \in E(G), \forall i \in[k], \\
& x_{v w}^{i} \leq x_{v v}^{i} & & \forall v \in V(G), \forall w \in \bar{N}^{+}(v), \forall i \in[k], \\
& x_{u v}^{i} \in\{0,1\} & & \forall v \in V(G), \forall u \in \bar{N}^{-}[v], \forall i \in[k] .
\end{array}
$$

The objective function (7.1) minimizes the number of nonempty color classes. Constraints (7.2) ensure that each vertex $v$ of $G$ participates in at least $k$ (nonempty) color classes, considering color classes represented by $v$ or by any of its negative anti-neighbors. Constraints (7.3) guarantee that at most one endpoint of each edge belongs to the $i$-th color class represented by a vertex $v$ of $G$. Moreover, if this color class is empty, it cannot include any vertex according to constraints (7.4).

As in [16], we shall assume, without loss of generality, that $G$ has neither universal nor dominated vertex, provided that this kind of vertices could be removed from $G$. Indeed, if a vertex $v$ is universal $\left(\bar{N}^{-}(v)=\bar{N}^{+}(v)=\emptyset\right)$, then $\chi_{k}(G)=\chi_{k}(G-v)+k$. If $v$ is dominated $(\bar{N}[v] \supseteq \bar{N}[u]$ for some $u \in V(G))$, then $\chi_{k}(G)=\chi_{k}(G-v)$.

\subsection{Definition and basic properties}

Let $M=\left\{v: \bar{N}^{-}(v)=\emptyset\right\}$ be the set of sources or minimal vertices in the ordering $\prec$. Let $V^{\prime}=$ $V(G) \backslash M$ be the set of non-minimal vertices, $n^{\prime}=\left|V^{\prime}\right|$ and $\eta=n^{\prime}+\bar{m}$. By Inequalities (7.2), we have that $x_{v v}^{i}=1$, for all $v \in M$ and $i \in[k]$. Since these variables can be removed from formulation $\mathcal{R}_{k}(G)$, we define its corresponding polytope as

$$
\mathcal{P}_{k}(G)=\operatorname{conv}\left\{x \in \mathbb{B}^{k\left(n^{\prime}+\bar{m}\right)}:(\mathbf{1}, x) \in \mathbb{B}^{k(n+\bar{m})} \text { satisfies }(7.2)-(7.5)\right\},
$$

where vector 1 comprises the entries indexed by $i \in[k]$ and $v \in M$.

For instance, consider the graph $G=C_{5}$ illustrated in Figure 6.1 and the vertex order $v_{1} \prec v_{2} \prec v_{3} \prec$ $v_{4} \prec v_{5}$. As $M=\left\{v_{1}, v_{2}\right\}$, every vector $x \in \mathcal{P}_{2}(G)$ comprises only the entries $x_{v_{j} v_{j}}$ for $i \in\{1,2\}$ and $j \in\{3,4,5\}$, and $x_{v_{j} v_{\ell}}^{i}$ for $i \in\{1,2\}$ and $(j, \ell) \in\{(1,3),(1,4),(2,4),(2,5),(3,5)\}$. The 2-fold 5-coloring depicted in Figure 6.1(b) can be represented, for example, by the vector $x$ whose non-null entries are $x_{v_{1} v_{3}}^{1}, x_{v_{1} v_{4}}^{2}, x_{v_{2} v_{4}}^{1}, x_{v_{2} v_{5}}^{2}, x_{v_{3} v_{3}}^{1}$ and $x_{v_{3} v_{5}}^{1}$. If we assign a third (new) color to $v_{4}$, this can be indicated by either setting $x_{v_{4} v_{4}}^{1}=1$ or $x_{v_{4} v_{4}}^{2}=1$.

Note that, given a vertex $v$ of $G$, Inequality (7.2) corresponding to $v$ is the summation of the constraints

$$
\sum_{u \in \bar{N}^{-}-[v]} x_{u v}^{i} \geq 1 \quad \text { for all } i \in[k] .
$$

Moreover, for a fixed $i \in[k]$, the convex hull of the points satisfying (7.3)-(7.5) and (7.6) for all $v \in V(G)$ 
gives $\mathcal{P}_{1}(G)$. Using these remarks and the fact that $\operatorname{conv}(X \times Y)=\operatorname{conv}(X) \times \operatorname{conv}(Y)$, we obtain that

$$
\mathcal{P}_{k}(G) \supseteq \underbrace{\mathcal{P}_{1}(G) \times \cdots \times \mathcal{P}_{1}(G)}_{k \text { times }}
$$

More generally, we have the following property.

Lemma 7.1. For every $k, \ell \in \mathbb{N}, \mathcal{P}_{k}(G) \times \mathcal{P}_{\ell}(G) \subseteq \mathcal{P}_{k+\ell}(G)$.

Proof. Let $x=(y, z) \in Y \times Z$, where $Y=\mathcal{P}_{k}(G) \cap \mathbb{B}^{k \eta}$ and $Z=\mathcal{P}_{\ell}(G) \cap \mathbb{B}^{\ell \eta}$. We have that $y$ and $z$ satisfy constraints $(7.2)-(7.5)$ of $\mathcal{R}_{k}(G)$ and $\mathcal{R}_{\ell}(G)$, respectively. Observe that $x_{u v}^{i}=y_{u v}^{i}$ for $i \in[k]$, and $x_{u v}^{k+i}=z_{u v}^{i}$ for $i \in[\ell]$. Thus, $x$ satisfies constraints $(7.3)-(7.5)$ of $\mathcal{R}_{k+\ell}(G)$ and

$$
\sum_{i=1}^{k+\ell} \sum_{u \in \bar{N}^{-}[v]} x_{u v}^{i}=\sum_{i=1}^{k} \sum_{u \in \bar{N}^{-}[v]} y_{u v}^{i}+\sum_{i=1}^{\ell} \sum_{u \in \bar{N}^{-}[v]} z_{u v}^{i} \geq k+\ell, \quad \text { for all } v \in V(G)
$$

In other terms, $x \in X=\mathcal{P}_{k+\ell}(G) \cap \mathbb{B}^{(k+\ell) \eta}$, and so $Y \times Z \subseteq X$. This leads to

$$
\mathcal{P}_{k}(G) \times \mathcal{P}_{\ell}(G)=\operatorname{conv}(Y) \times \operatorname{conv}(Z) \subseteq \operatorname{conv}(X)=\mathcal{P}_{k+\ell}(G)
$$

Proposition 7.2. For every $k \in \mathbb{N}, \mathcal{P}_{k}(G)$ is full-dimensional.

Proof. It is known that $\mathcal{P}_{1}(G)$ has dimension $\eta=n^{\prime}+\bar{m}[16]$. By (7.7), we conclude that the dimension of $\mathcal{P}_{k}(G)$ is $k \eta$.

Given $x \in \mathcal{P}_{k}(G)$ and $i \in[k]$, let $x^{i}$ denote the subvector of $x$ defined by the entries $x_{u v}^{i}$, for $v \in V^{\prime}$, $u \in \bar{N}^{-}[v]$. In addition, let $x_{u}^{i}$ denote the subvector of $x^{i}$ with entries $x_{u v}^{i}$, for fixed $u \in V$. Henceforth, we assume that the entries in a vector $x \in \mathcal{P}_{k}(G)$ are organized in the form $x=\left(x^{1}, x^{2}, \ldots, x^{k}\right)$. An inequality $\lambda x \leq \lambda_{0}$ will be denoted by $\left(\lambda, \lambda_{0}\right)$ and entries in $\lambda$ are indexed as in $x$.

Lemma 7.3. Let $k \in \mathbb{N}, j, \ell \in[k]$ and $u \in V^{\prime}$. If $\left(\lambda, \lambda_{0}\right) \in \mathbb{R}^{k \eta} \times \mathbb{R}$ defines a face of $\mathcal{P}_{k}(G)$, then $\left(\hat{\lambda}, \lambda_{0}\right) \in$ $\mathbb{R}^{k \eta} \times \mathbb{R}$ defines a face of $\mathcal{P}_{k}(G)$ of the same dimension, where $\hat{\lambda}$ is obtained from $\lambda$ by interchanging $\lambda_{u}^{j}$ and $\lambda_{u}^{\ell}$.

Proof. Let $\lambda \in \mathbb{R}^{k \eta}$ be a row-vector describing the left-hand side of a valid inequality of $\mathcal{P}_{k}(G)$ and $\hat{\lambda}$ be the vector obtained from $\lambda$ by interchanging $\lambda_{u}^{j}$ and $\lambda_{u}^{\ell}$. Similarly, let $x \in \mathcal{P}_{k}(G)$ such that $\lambda x=\lambda_{0}$, and $\hat{x}$ be the vector obtained from $x$ by interchanging $x_{u}^{j}$ and $x_{u}^{\ell}$. Note that, for any $i \in[k], x_{u}^{i}$ describes the $i$-th color class represented by $u$ (which is empty, if $x_{u}^{i}=\mathbf{0}$ ). Therefore, $\hat{x}$ describes the same $k$-fold coloring as $x$. Since $\hat{\lambda} \hat{x}=\lambda x=\lambda_{0}$ and $\hat{x}$ also belongs to $\mathcal{P}_{k}(G)$, we get that $\hat{x}$ belongs to the face defined by $\left(\hat{\lambda}, \lambda_{0}\right)$, and the result follows.

Example 7.4. Let $G$ be a cycle on 5 vertices, where $V(G)=\left\{v_{1}, \ldots, v_{5}\right\}$ and $E(G)=\left\{v_{i} v_{i+1}: i \in\right.$ $\{1, \ldots, 4\}\} \cup\left\{v_{5} v_{1}\right\}$. Let $\prec$ be such that $v_{i} \prec v_{i+1}$. Note that $V^{\prime}=\left\{v_{3}, v_{4}, v_{5}\right\}$ and $\chi_{2}(G)=5$ [18]. One may easily check that $x_{33}^{1}+x_{44}^{1}+x_{55}^{1}+x_{33}^{2}+x_{44}^{2} \geq 1$ is a valid inequality for $\mathcal{P}_{2}(G)$. Indeed, if the left-hand side could be zero, we would get by (7.4) that $x_{35}^{1}=x_{35}^{2}=0$, and by (7.2)-(7.3) that $x_{13}^{1}=x_{13}^{2}=1$, $x_{14}^{1}=x_{14}^{2}=0, x_{24}^{1}=x_{24}^{2}=1, x_{25}^{1}=x_{25}^{2}=0$, and then the contradiction $x_{55}^{2} \geq 2$. Actually, we show in Example 8.6 that this inequality defines a facet of $\mathcal{P}_{2}(G)$. By Lemma 7.3, $x_{33}^{1}+x_{44}^{1}+x_{33}^{2}+x_{44}^{2}+x_{55}^{2} \geq 1$ is also valid and facet-defining for $\mathcal{P}_{2}(G)$. 
For ease of notation, in the next chapters, we partition each subvector $x^{i}$ into $\left(x_{V^{\prime}}^{i}, x_{\bar{E}}^{i}\right)$, where $x_{V^{\prime}}^{i}$ and $x_{\bar{E}}^{i}$ respectively comprise the entries $x_{v v}^{i}$ and $x_{u v}^{i}$, for all $v \in V^{\prime}$ and all $u \in \bar{N}^{-}(v)$. In addition, for $v \in V^{\prime}$ and $u \in \bar{N}^{-}(v)$, let $e^{v} \in \mathbb{B}^{n^{\prime}}$ and $e^{u v} \in \mathbb{B}^{\bar{m}}$, be the unit vectors with 1 only in the entries indexed by $v \in V^{\prime}$ and $u v \in E(\bar{G})$, respectively. The vectors $e^{V^{\prime}}=\sum_{v \in V^{\prime}} e^{v}$ and $e^{\bar{E}}=\sum_{u v \in E(\bar{G})} e^{u v}$ have all entries equal to 1 . For each $v \in V^{\prime}$, let $r_{v}$ denote a vertex that may represent $v$, that is, $r_{v} \in \bar{N}^{-}(v)$. 


\section{Chapter 8}

\section{Inherited facets}

In this chapter, we study the relationship between two representatives $k$-fold coloring polytopes and between the representatives $k$-fold coloring polytope and the stable set polytope.

First, we profit from the known facial studies for $\mathcal{P}_{1}(G)$ in two ways. In Section 8.1, we present a lifting theorem that combines a facet of $\mathcal{P}_{k}(G)$ and a facet of $\mathcal{P}_{\ell}(G)$ into a facet of $\mathcal{P}_{k+\ell}(G)$, and we also show specific liftings from facets of $\mathcal{P}_{k}(G)$ to facets of $\mathcal{P}_{k+1}(G)$. In Section 8.2, we show how to project facets from $\mathcal{P}_{1}\left(G \circ K_{k}\right)$ into facets of $\mathcal{P}_{k}(G)$.

In Section 8.3, we show how to transform to $\mathcal{P}_{k}(G)$ any facet-defining inequality of the stable set polytope associated with the subgraph of $G$ induced by the anti-neighborhood of each vertex.

\subsection{Facets from $\mathcal{P}_{k}(G)$ to $\mathcal{P}_{k+\ell}(G)$}

Some facet-defining inequalities of $\mathcal{P}_{k}(G)$ can be lifted to generate facets of $\mathcal{P}_{k+\ell}(G)$. Sometimes, we can carry these inequalities from $k=1$ to all $\ell \geq 1$. Starting from the following basic property, we show how to obtain these liftings. In particular, they are used to prove that the cover inequalities (7.2) and some of the bounding inequalities from (7.5), i.e. inequalities $x_{u v}^{i} \geq 0$ and $x_{u v}^{i} \leq 1$ for $v \in V(G)$, $u \in \bar{N}^{-}[v]$ and $i \in[k]$, are facet-defining.

Lemma 8.1. Let $k, \ell \in \mathbb{N}$. Let $F_{k}, F_{\ell}$ and $F_{k+\ell}$ be faces of $\mathcal{P}_{k}(G), \mathcal{P}_{\ell}(G)$ and $\mathcal{P}_{k+\ell}(G)$, respectively, given by

- $F_{k}=\left\{y \in \mathcal{P}_{k}(G): \lambda y=\lambda_{0}\right\}$

- $F_{\ell}=\left\{z \in \mathcal{P}_{\ell}(G): \pi z=\pi_{0}\right\}$, and

- $F_{k+\ell}=\left\{(y, z) \in \mathcal{P}_{k+\ell}(G): \lambda y=\lambda_{0}\right.$ and $\left.\pi z=\pi_{0}\right\}$,

where $\left(\lambda, \lambda_{0}\right) \in \mathbb{R}^{k \eta} \times \mathbb{R},\left(\pi, \pi_{0}\right) \in \mathbb{R}^{\ell \eta} \times \mathbb{R}$ and $\left(\lambda, \pi, \lambda_{0}+\pi_{0}\right) \in \mathbb{R}^{k \eta} \times \mathbb{R}^{\ell \eta} \times \mathbb{R}$ do not need to define valid inequalities. There exists an affinely independent set with $\operatorname{dim}\left(F_{k}\right)+\operatorname{dim}\left(F_{\ell}\right)+1$ vectors of $F_{k} \times F_{\ell}$ that belong to $F_{k+\ell}$. Thus, $\operatorname{dim}\left(F_{k+\ell}\right) \geq \operatorname{dim}\left(F_{k}\right)+\operatorname{dim}\left(F_{\ell}\right)$. In fact, $\operatorname{dim}\left(F_{k+\ell}\right) \geq \operatorname{dim}\left(F_{k}\right)+\operatorname{dim}\left(F_{\ell}\right)+1$ if $F_{k+\ell} \not$ affine $\left(F_{k} \times F_{\ell}\right)$.

Proof. By Lemma 7.1 and the definitions of $F_{k}, F_{\ell}$ and $F_{k+\ell}$, we conclude that $F_{k} \times F_{\ell} \subseteq F_{k+\ell}$. Therefore, $\operatorname{dim}\left(F_{k+\ell}\right) \geq \operatorname{dim}\left(F_{k} \times F_{\ell}\right)=\operatorname{dim}\left(F_{k}\right)+\operatorname{dim}\left(F_{\ell}\right)$, and $F_{k+\ell}$ contains a set $W$ with $\operatorname{dim}\left(F_{k}\right)+\operatorname{dim}\left(F_{\ell}\right)+1$ affinely independent vectors in $F_{k} \times F_{\ell}$. In addition, if there exists a vector $\hat{x}=(\hat{y}, \hat{z})$ in $F_{k+\ell} \backslash$ affine $\left(F_{k} \times\right.$ $\left.F_{\ell}\right), W \cup\{\hat{x}\}$ is an affinely independent subset of $F_{k+\ell}$ with $\operatorname{dim}\left(F_{k}\right)+\operatorname{dim}\left(F_{\ell}\right)+2$ vectors. Therefore, $\operatorname{dim}\left(F_{k+\ell}\right)=\operatorname{affine}-\operatorname{rank}\left(F_{k+\ell}\right)-1 \geq \operatorname{dim}\left(F_{k}\right)+\operatorname{dim}\left(F_{\ell}\right)+1$. 
A special case of Lemma 8.1 occurs when an inequality defining a face of dimension at least $k \eta-2$ of $\mathcal{P}_{k}(G)$ is valid for $\mathcal{P}_{k+\ell}(G)$. This leads to the following trivial liftings.

Corollary 8.2. Let $k, \ell \in \mathbb{N}$, and let $\left(\lambda, \lambda_{0}\right) \in \mathbb{R}^{k \eta} \times \mathbb{R}$ be a facet-defining inequality of $\mathcal{P}_{k}(G)$. If $\left(\lambda, \mathbf{0}, \lambda_{0}\right) \in$ $\mathbb{R}^{k \eta} \times \mathbb{R}^{\ell \eta} \times \mathbb{R}$ is a valid inequality of $\mathcal{P}_{k+\ell}(G)$, then it defines a facet of $\mathcal{P}_{k+\ell}(G)$.

Proof. Let $F_{k}$ and $F_{k+\ell}$ be the facet of $\mathcal{P}_{k}(G)$ and the face of $\mathcal{P}_{k+\ell}(G)$ defined by $\left(\lambda, \lambda_{0}\right)$ and $\left(\lambda, \mathbf{0}, \lambda_{0}\right)$, respectively. By Lemma 8.1 and Proposition 7.2, we obtain $\operatorname{dim}\left(F_{k+\ell}\right) \geq \operatorname{dim}\left(F_{k}\right)+\operatorname{dim}\left(\mathcal{P}_{\ell}(G)\right)=$ $k \eta-1+\ell \eta=\operatorname{dim}\left(\mathcal{P}_{k+\ell}(G)\right)-1$. Since $F_{k+\ell}$ must be a proper face of $\mathcal{P}_{k+\ell}(G)$, it defines a facet of $\mathcal{P}_{k+\ell}(G)$.

Corollary 8.3. Let $k, \ell \in \mathbb{N}$, and let $\left(\lambda, \lambda_{0}\right) \in \mathbb{R}^{k \eta} \times \mathbb{R}$ define a proper face $F_{k}$ of $\mathcal{P}_{k}(G)$ with dimension at least $k \eta-2$. If $\left(\lambda, \mathbf{0}, \lambda_{0}\right) \in \mathbb{R}^{k \eta} \times \mathbb{R}^{\ell \eta} \times \mathbb{R}$ is a valid inequality of $\mathcal{P}_{k+\ell}(G)$ and there is a point $(y, z) \in \mathcal{P}_{k+\ell}(G)$ such that $y \notin \operatorname{affine}\left(F_{k}\right)$ and $\lambda y=\lambda_{0}$, then $\left(\lambda, \mathbf{0}, \lambda_{0}\right)$ defines a facet of $\mathcal{P}_{k+\ell}(G)$.

Proof. Let $F_{k}$ and $F_{k+\ell}$ be the proper faces of $\mathcal{P}_{k}(G)$ and $\mathcal{P}_{k+\ell}(G)$ defined by $\left(\lambda, \lambda_{0}\right)$ and $\left(\lambda, \mathbf{0}, \lambda_{0}\right)$, respectively. Note that affine $\left(F_{k} \times \mathcal{P}_{\ell}(G)\right) \subseteq$ affine $\left(F_{k}\right) \times \operatorname{affine}\left(\mathcal{P}_{\ell}(G)\right)$, and so $(y, z) \in F_{k+\ell} \backslash$ affine $\left(F_{k} \times\right.$ $\left.\mathcal{P}_{\ell}(G)\right)$. Thus, Lemma 8.1 ensures that $\operatorname{dim}\left(F_{k+\ell}\right) \geq \operatorname{dim}\left(F_{k}\right)+\operatorname{dim}\left(\mathcal{P}_{\ell}(G)\right)+1 \geq k \eta-2+\ell \eta+1=$ $\operatorname{dim}\left(\mathcal{P}_{k+\ell}(G)\right)-1$. Therefore, $\left(\lambda, \mathbf{0}, \lambda_{0}\right)$ defines a facet of $\mathcal{P}_{k+\ell}(G)$.

Using the above stated trivial liftings, we show the bounding inequalities from (7.5) that define facets of $\mathcal{P}_{k}(G)$.

Proposition 8.4. Let $k \in \mathbb{N}$ and $i \in[k]$. The following inequalities are facet-defining for $\mathcal{P}_{k}(G)$ :

1. $x_{u u}^{i} \leq 1$, for all $u \in V^{\prime}$;

2. $x_{u u}^{i} \geq 0$, for all $u \in V^{\prime}$ such that $\bar{N}^{+}(u)=\emptyset$;

3. $x_{u v}^{i} \geq 0$, for all $v \in V^{\prime}$ and $u \in \bar{N}^{-}(v)$, with $\left|\bar{N}^{-}(v)\right| \geq 2$ when $k=1$.

Proof. The statement is known to be valid for $k=1[16]$. We now prove that $x_{u v}^{1} \geq 0$ defines a facet of $\mathcal{P}_{2}(G)$ if $\bar{N}^{-}(v)=\{u\}$. The result then follows by Corollary 8.2 and Lemma 7.3.

Let $(\lambda, 0) \in \mathbb{R}^{\eta} \times \mathbb{R}$ and $(\lambda, \mathbf{0}, 0) \in \mathbb{R}^{\eta} \times \mathbb{R}^{\eta} \times \mathbb{R}$ denote the valid inequality $-x_{u v}^{1} \leq 0$ for the polytopes $\mathcal{P}_{1}(G)$ and $\mathcal{P}_{2}(G)$, respectively. Let $F_{1}$ and $F_{2}$ be the corresponding faces. We first exhibit $\eta-1$ vectors in $F_{1}$. They are:

$$
\left(e^{V^{\prime}}, \mathbf{0}\right) ; \quad\left(e^{V^{\prime}}, e^{w z}\right) \forall z \in V^{\prime} \backslash\{v\} \forall w \in \bar{N}^{-}(z) ; \quad\left(e^{V^{\prime}}-e^{z}, e^{r_{z} z}\right) \forall z \in V^{\prime} \backslash\{v\} .
$$

The first vector describes the trivial coloring, where each vertex induces a singleton color class. In the second group, we extend color class $\{w\}$ in the trivial coloring to $\{w, z\}$ which is represented by $w$. In the third group, we replace, in the trivial coloring, the color class $\{z\}$ by $\left\{r_{z}, z\right\}$ whose representative is $r_{z}$. So, it is easy to see that all these vectors describe 1-fold colorings where $u$ does not represent $v$, that is, they are points in $F_{1}$. Moreover, they form an affinely independent set. Thus, we have $\operatorname{dim}\left(F_{1}\right) \geq \eta-2$. In addition, vector $\hat{x}=\left(\hat{x}^{1}, \hat{x}^{2}\right)$, where $\hat{x}^{1}=\left(e^{V^{\prime}}-e^{v}, \mathbf{0}\right)$ and $\hat{x}^{2}=\left(e^{V^{\prime}}, e^{u v}\right)$, describes a 2-fold coloring in which $\hat{x}_{u v}^{1}=0$, hence, $\hat{x} \in F_{2}$. Note that $\hat{x}_{v v}^{1}=0$. On the other hand, every $x^{1} \in F_{1}$ must satisfy $x_{v v}^{1}=1$ since $x_{u v}^{1}=0$ and $\bar{N}^{-}(v)=\{u\}$. Therefore, we conclude that $\hat{x}^{1} \notin$ affine $\left(F_{1}\right)$. By Corollary 8.3, it follows that $F_{2}$ is a facet of $\mathcal{P}_{2}(G)$. 
Observe that the sufficient conditions stated in Proposition 8.4 are also necessary to define the corresponding facets. Indeed, if $v \in \bar{N}^{+}(u)$, then $x_{u u}^{i} \geq x_{u v}^{i} \geq 0$ dominates $x_{u u}^{i} \geq 0$. Similarly, if $k=1$ and $\bar{N}^{-}(v)=\{u\}$, constraint $(7.2)$ of $\mathcal{R}_{k}(G)$ for $v$ becomes $x_{u v}^{1} \geq 1-x_{v v}^{1} \geq 0$, which dominates $x_{u v}^{1} \geq 0$.

Lemma 8.1 also provides conditions for obtaining a facet-defining inequality of $\mathcal{P}_{k+\ell}(G)$ from facetdefining inequalities of $\mathcal{P}_{k}(G)$ and $\mathcal{P}_{\ell}(G)$.

Corollary 8.5. Let $k, \ell, r \in \mathbb{N}$. Let $\left(\lambda, \lambda_{0}\right) \in \mathbb{R}^{k \eta} \times \mathbb{R}$ be a facet-defining inequality for $\mathcal{P}_{k}(G)$ and let $\left(\pi^{i}, \pi_{0}^{i}\right) \in \mathbb{R}^{\ell \eta} \times \mathbb{R}$ be different facet-defining inequalities for $\mathcal{P}_{\ell}(G)$, for all $i \in[r]$. Define $\pi=\sum_{i=1}^{r} \pi^{i}$ and $\pi_{0}=\sum_{i=1}^{r} \pi_{0}^{i}$. If $\left(\lambda, \pi, \lambda_{0}+\pi_{0}\right)$ is valid for $\mathcal{P}_{k+\ell}(G)$ and there exist points $\bar{w}^{i}=\left(\bar{y}^{i}, \bar{z}^{i}\right) \in \mathcal{P}_{k+\ell}(G)$ such that, for every $i \in[r]$,

- $\lambda \bar{y}^{i}+\pi \bar{z}^{i}=\lambda_{0}+\pi_{0}$,

- $\pi^{i} \bar{z}^{i} \neq \pi_{0}^{i}$, and

- $\pi^{j} \bar{z}^{i}=\pi_{0}^{j}$, for every $j \in\{i+1, \ldots, r\}$,

then $\left(\lambda, \pi, \lambda_{0}+\pi_{0}\right)$ is facet-defining for $\mathcal{P}_{k+\ell}(G)$

Proof. We have that $F_{k}=\left\{y \in \mathcal{P}_{k}(G): \lambda y=\lambda_{0}\right\}$ is a $(k \eta-1)$-dimensional face of $\mathcal{P}_{k}(G)$ and $F_{\ell}=\{z \in$ $\left.\mathcal{P}_{\ell}(G): \pi^{i} z=\pi_{0}^{i}, i \in[r]\right\}$ is an $(\ell \eta-r)$-dimensional face of $\mathcal{P}_{\ell}(G)$. Due to the validity of the inequalities, one may easily check that $F_{\ell}=\left\{z \in \mathcal{P}_{\ell}(G): \pi z=\pi_{0}\right\}$. Let $F_{k+\ell}=\left\{(y, z) \in \mathcal{P}_{k+\ell}(G): \lambda y+\pi z=\lambda_{0}+\pi_{0}\right\}$ be the resulting proper face of $\mathcal{P}_{k+\ell}(G)$. By Lemma 8.1, $F_{k+\ell}$ contains a set $W_{0} \subseteq F_{k} \times F_{\ell}$ with $(k+\ell) \eta-r$ affinely independent vectors.

For each $i \in[r]$, we define $W_{i}=W_{i-1} \cup\left\{\bar{w}^{i}\right\}$. Note that $W_{i-1}$ is contained in the affine set

$$
S_{i}:=\left\{(y, z) \in \mathbb{R}^{k \eta} \times \mathbb{R}^{\ell \eta}: \pi^{j} z=\pi_{0}^{j}, \text { for every } j \in\{i, i+1, \ldots, r\}\right\}
$$

and $\bar{w}^{i} \in F_{k+\ell} \backslash S_{i}$. Thus, $\bar{w}^{i} \notin$ affine $\left(W_{i-1}\right)$. This implies that $W_{i}$ is affinely independent if so is $W_{i-1}$. Since $W_{0}$ is affinely independent, we conclude that $W_{i}$ is also affinely independent, for every $i \in[r]$. In particular, $W_{r}$ is a set with $(k+\ell) \eta$ affinely independent points of $F_{k+\ell}$. Therefore, $F_{k+\ell}$ is a facet of $P_{k+\ell}(G)$.

Example 8.6 (Cont.). Using Corollary 8.5, we now show that $x_{33}^{1}+x_{44}^{1}+x_{55}^{1}+x_{33}^{2}+x_{44}^{2} \geq 1$ is facetdefining for $\mathcal{P}_{2}(G)$. Let $\left(\lambda, \lambda_{0}\right),\left(\pi^{1}, \pi_{0}^{1}\right)$ and $\left(\pi^{2}, \pi_{0}^{2}\right)$ correspond to the inequalities $-x_{33}^{1}-x_{44}^{1}-x_{55}^{1} \leq-1$, $x_{55}^{2} \leq 1$ and $-x_{33}^{2}-x_{44}^{2}-x_{55}^{2} \leq-1$, respectively. Note that the claimed facet is induced by $\left(\lambda, \pi^{1}+\pi^{2}, \lambda_{0}+\right.$ $\left.\pi_{0}^{1}+\pi_{0}^{2}\right)$.

It is known that $\left(\lambda, \lambda_{0}\right)$ (and equivalently $\left(\pi^{2}, \pi_{0}^{2}\right)$ ) is facet-defining for $\mathcal{P}_{1}(G)$ [16]. It is the internal odd cycle inequality. Actually, it is a particular inequality of the family of facet defining-inequalities given by Theorem 9.2 in Section 9.1. Proposition 8.4 ensures that $\left(\pi^{1}, \pi_{0}^{1}\right)$ is facet-defining for $\mathcal{P}_{1}(G)$ too. Following Corollary 8.5 to get the claimed result, it suffices to exhibit 2-fold colorings $w^{1}=\left(y^{1}, z^{1}\right)$ and $w^{2}=\left(y^{2}, z^{2}\right)$ such that $\pi^{1} z^{1} \neq \pi_{0}^{1}, \pi^{2} z^{1}=\pi_{0}^{2}$ and $\pi^{2} z^{2} \neq \pi_{0}^{2}$. For instance, consider the vectors defined by

$$
\begin{aligned}
& y^{1}=\left(0, e^{v_{1} v_{3}}+e^{v_{2} v_{4}}\right), z^{1}=\left(e^{v_{3}}, e^{v_{1} v_{4}}+e^{v_{2} v_{5}}+e^{v_{3} v_{5}}\right), \\
& y^{2}=\left(e^{v_{3}}, e^{v_{1} v_{3}}+e^{v_{2} v_{4}}+e^{v_{3} v_{5}}\right), \quad z^{2}=\left(0, e^{v_{1} v_{4}}+e^{v_{2} v_{5}}\right) .
\end{aligned}
$$

Observe that both $w_{1}$ and $w_{2}$ describe the 2-fold coloring of $C_{5}$ illustrated in Figure 6.1, whose color classes are $\left\{v_{1}, v_{3}\right\},\left\{v_{1}, v_{4}\right\},\left\{v_{2}, v_{4}\right\},\left\{v_{2}, v_{5}\right\},\left\{v_{3}, v_{5}\right\}$. 
Using Corollary 8.5, we next prove that inequalities (7.2) are facet-defining.

Proposition 8.7. Let $k \in \mathbb{N}$. For every $v \in V^{\prime}$, the following inequality is facet-defining for $\mathcal{P}_{k}(G)$ :

$$
\sum_{i=1}^{k} \sum_{u \in \bar{N}^{-}[v]} x_{u v}^{i} \geq k
$$

Proof. We use induction on $k$. Consider a vertex $v \in V^{\prime}$. Let $F_{1}=\left\{x \in \mathcal{P}_{1}(G): \pi x=\pi_{0}\right\}$ be the face of $\mathcal{P}_{1}(G)$ induced by inequality $-\sum_{u \in \bar{N}^{-}[v]} x_{u v}^{1} \leq-1$. It is known that $F_{1}$ is a facet of $\mathcal{P}_{1}(G)[16]$. Let $F_{k}=\left\{x \in \mathcal{P}_{k}(G): \lambda x=\lambda_{0}\right\}$ be the face of $\mathcal{P}_{k}(G)$ induced by inequality $-\sum_{i=1}^{k} \sum_{u \in \bar{N}^{-}[v]} x_{u v}^{i} \leq-k$. Suppose that $F_{k}$ is a facet of $\mathcal{P}_{k}(G)$. Clearly, inequality $\left(\lambda, \pi, \lambda_{0}+\pi_{0}\right)$ is valid for $\mathcal{P}_{k+1}(G)$. Let $F_{k+1}$ be the face of $\mathcal{P}_{k+1}(G)$ induced by $\left(\lambda, \pi, \lambda_{0}+\pi_{0}\right)$. Due to Corollary 8.5, it suffices to show a vector $(\hat{y}, \hat{z}) \in F_{k+1}$ such that $\pi \hat{z} \neq \pi_{0}$ to prove that $F_{k+1}$ is a facet of $\mathcal{P}_{k+1}(G)$. This vector is

$$
\hat{y}=(\underbrace{\left(e^{V^{\prime}}, \mathbf{0}\right), \ldots,\left(e^{V^{\prime}}, \mathbf{0}\right)}_{k-1 \text { times }},\left(e^{V^{\prime}}, e^{r_{v} v}\right)), \quad \hat{z}=\left(e^{V^{\prime}}-e^{v}, \mathbf{0}\right) .
$$

Note that $(\hat{y}, \hat{z})$ describes a $(k+1)$-fold coloring in which every vertex is colored exactly $k+1$ times. Moreover, each vertex represents its own color classes except vertex $v$ that has one of its colors represented by $r_{v}$, and receives no color in $\hat{z}$. Therefore, we have $(\hat{y}, \hat{z}) \in F_{k+1}$ and $\pi \hat{z}=0>-1=\pi_{0}$.

Differently from what Corollary 8.5 suggests, we may consider nontrivial combinations of two facetdefining inequalities of $\mathcal{P}_{k}(G)$ and $\mathcal{P}_{\ell}(G)$ in order to get a facet-defining inequality of $\mathcal{P}_{k+\ell}(G)$. To show the proper combinations, we need the following definitions, based on the approach presented in [79].

Definition 8.8. Let $\tau$ be a positive integer, let $P \subseteq \mathbb{R}^{\tau}$ be a polytope and let $\Lambda: \mathbb{R}^{\tau} \rightarrow \mathbb{R}$, and $\Pi: \mathbb{R}^{\tau} \rightarrow \mathbb{R}$ be two affine functions. A reference point, in $P$, for $\Lambda$ with respect to $\Pi$, to be denoted by $x(\Lambda ; \Pi)$, is a vector $\bar{x} \in P$ such that $\Lambda(\bar{x}) \neq 0$ or $\Pi(\bar{x}) \neq 0$, and

$$
\Lambda(\bar{x}) \Pi(x) \leq \Lambda(x) \Pi(\bar{x}), \quad \text { for all } x \in P \text {. }
$$

Remark 8.9. It holds that $x(\Lambda ; \Pi)=x(-\Lambda ;-\Pi)=x(-\Pi ; \Lambda)=x(\Pi ;-\Lambda)$.

Remark 8.10. As Inequality (8.1) is linear, it is equivalent to require it to hold only for the vertices of $P$.

Note that a reference point, if it exists, defines a valid inequality. The existence of such points are guaranteed under some conditions, which include for instance the case where either $\Lambda(x) \leq 0$ or $\Pi(x) \leq 0$ is valid.

Lemma 8.11. Let $\tau$ be a positive integer, let $P \subseteq \mathbb{R}^{\tau}$ be a full-dimensional polytope, and let $\Lambda: \mathbb{R}^{\tau} \rightarrow \mathbb{R}$ and $\Pi: \mathbb{R}^{\tau} \rightarrow \mathbb{R}$ be two affine functions. There exists a reference point $\bar{x}$ in $P$ for $\Lambda$ with respect to $\Pi$ if and only if one of the following conditions holds:

1. $\Lambda(x) \leq 0$ or $\Pi(x) \leq 0$, for every $x \in P$; or

2. $\Lambda(x) \leq 0$ or $\Pi(x) \geq 0$, for every $x \in P$; or

3. $\Lambda(x) \geq 0$ or $\Pi(x) \leq 0$, for every $x \in P$; or

4. $\Lambda(x) \geq 0$ or $\Pi(x) \geq 0$, for every $x \in P$. 
Proof. Firstly, suppose that $\Lambda(x) \geq 0$ is valid for $P$. If there is $\bar{x} \in P$ such that $\Lambda(\bar{x})=0$ and $\Pi(\bar{x})>0$, then $\bar{x}$ is clearly a reference point $x(\Lambda, \Pi)$. Thus, suppose that $\Lambda(x) \neq 0$ or $\Pi(x) \leq 0$, for every $x \in P$. Let $X$ be the (finite) set of vertices of $P$. Since $P$ is full-dimensional and $\Lambda(x) \geq 0$ is valid, there is $x \in X$ such that $\Lambda(x)>0$. Choose $\bar{x} \in X$ such that

$$
\frac{\Pi(\bar{x})}{\Lambda(\bar{x})}=\max _{x \in X}\left\{\frac{\Pi(x)}{\Lambda(x)}: \Lambda(x)>0\right\} .
$$

Consider $x \in X$ and note that $\Lambda(x) \geq 0$ and $\Lambda(\bar{x})>0$. If $\Lambda(x)=0$, then $\Pi(x) \leq 0$, and so Inequality (8.1) holds. If $\Lambda(x)>0$, then $\frac{\Pi(\bar{x})}{\Lambda(\bar{x})} \geq \frac{\Pi(x)}{\Lambda(x)}$, and the inequality holds again. Note that $\Lambda(\bar{x}) \neq 0$ or $\Pi(\bar{x}) \neq 0$. Thus, by Remark 8.10, it follows that $\bar{x}$ is a reference point $x(\Lambda ; \Pi)$.

Since no assumption was made on $\Pi$ (besides being affine), we have also proved the existence of $x(\Lambda ;-\Pi)$ if $\Lambda(x) \geq 0$ is valid. Interchanging the roles of $\Lambda$ and $\Pi$, we also prove that there exist reference points $x(\Pi ; \Lambda)$ and $x(\Pi ;-\Lambda)$ if $\Pi(x) \geq 0$ is valid for $P$. Alternatively, replacing $\Lambda$ by $-\Lambda$ (resp. $\Pi$ by $-\Pi)$, we obtain a reference point $x(-\Lambda ;-\Pi)(\operatorname{resp} . x(-\Pi ; \Lambda))$ if $\Lambda(x) \leq 0(\operatorname{resp} . \Pi(x) \leq 0)$ is valid for $P$. By Remark 8.9, we conclude that there exist a reference point $\bar{x}=x(\Lambda ; \Pi)$, if one of the inequalities $\Lambda(x) \geq 0, \Lambda(x) \leq 0, \Pi(x) \geq 0$ or $\Pi(x) \leq 0$ is valid for $P$.

Now, suppose that none of these four inequalities is valid for $P$. Consider a vector $\bar{x} \in X$ defined as in (8.2). Again, it is well-defined because $\Lambda(x) \leq 0$ is not valid for $P$. Suppose that Hypothesis 1 holds, that is, $\Lambda(x) \leq 0$ or $\Pi(x) \leq 0$, for every $x \in P$. It follows that

$$
\Lambda(\bar{x})>0 \quad \text { and } \quad \Pi(\bar{x}) \leq 0 .
$$

Given $x \in X$, consider the following three complementary cases:

(i). $\Lambda(x)>0$ : By Property (8.2) of $\bar{x}$, we have that $\frac{\Pi(\bar{x})}{\Lambda(\bar{x})} \geq \frac{\Pi(x)}{\Lambda(x)}$ or still $\Lambda(\bar{x}) \Pi(x) \leq \Lambda(x) \Pi(\bar{x})$.

(ii). $\Lambda(x) \leq 0$ and $\Pi(x) \leq 0$ : Clearly, we have $\Lambda(\bar{x}) \Pi(x) \leq 0 \leq \Lambda(x) \Pi(\bar{x})$.

(iii). $\Lambda(x) \leq 0$ and $\Pi(x)>0$ : By the opposite signs of the affine functions $\Lambda$ and $\Pi$ at $x$ and $\bar{x}$, there exists a point $\hat{x}$ in the open segment $(\bar{x}, x)$ such that $\Lambda(\hat{x})=\Pi(\hat{x})$ (see Figure 8.1(a)). Indeed, $\hat{x}=\alpha x+(1-\alpha) \bar{x}$, where

$$
\alpha=\frac{\Lambda(\bar{x})-\Pi(\bar{x})}{\Pi(x)-\Lambda(x)+\Lambda(\bar{x})-\Pi(\bar{x})} \in(0,1) .
$$

Since $P$ is convex, $\hat{x} \in P$. So, Hypothesis 1 ensures that $\Lambda(\hat{x})=\Pi(\hat{x}) \leq 0$. Consider the affine function $\Theta(w)=\Lambda(\bar{x}) \Pi(w)-\Lambda(w) \Pi(\bar{x})$. We shall prove that $\Theta(x) \leq 0$. Note that $x=\frac{1}{\alpha} \hat{x}+\frac{\alpha-1}{\alpha} \bar{x}$ and $\Theta(\bar{x})=0$. Therefore, $\Theta(x)=\frac{1}{\alpha} \Theta(\hat{x}) \leq 0$, where the inequality is due to case (ii) and $\alpha>0$.

By Remark 8.10, it follows that $\bar{x}$ is a reference point $x(\Lambda, \Pi)$. Similarly, we prove the existence of $x(\Pi, \Lambda)$ under Hypothesis 1. More generally, under Hypothesis 2, 3 or 4, one may prove that there is a reference point $x(-\Pi, \Lambda), x(\Pi,-\Lambda)$ or $x(-\Lambda,-\Pi)$, respectively. By Remark 8.9, we have a reference point $\tilde{x}=$ $x(\Lambda, \Pi)$ in any case.

To show the converse, consider a reference point $\bar{x}=x(\Lambda ; \Pi)$. By definition, it holds that $\Lambda(\bar{x}) \neq 0$ or $\Pi(\bar{x}) \neq 0$. Suppose to the contrary that none of the conditions $1-4$ in the statement holds. Therefore, there exist $x^{1}, x^{2}, x^{3}$ and $x^{4}$ in $P$ such that $\Lambda\left(x^{1}\right)>0, \Pi\left(x^{1}\right)>0, \Lambda\left(x^{2}\right)>0, \Pi\left(x^{2}\right)<0, \Lambda\left(x^{3}\right)<0$, $\Pi\left(x^{3}\right)>0, \Lambda\left(x^{4}\right)<0$ and $\Pi\left(x^{4}\right)<0$ (see Figure 8.1(b)). This implies that none of the inequalities $\Lambda(x) \leq 0, \Lambda(x) \geq 0, \Pi(x) \leq 0$ and $\Pi(x) \geq 0$ is valid for $P$. Then, by (8.1), we must have $\Lambda(\bar{x}) \neq 0$ 
and $\Pi(\bar{x}) \neq 0$. If $\Lambda(\bar{x}) \Pi(\bar{x})>0$, Inequality (8.1) is violated by $x^{2}$ or $x^{3}$. If $\Lambda(\bar{x}) \Pi(\bar{x})<0$, Inequality (8.1) is violated by $x^{1}$ or $x^{4}$. In any case, we reach a contradiction, and therefore the result follows.

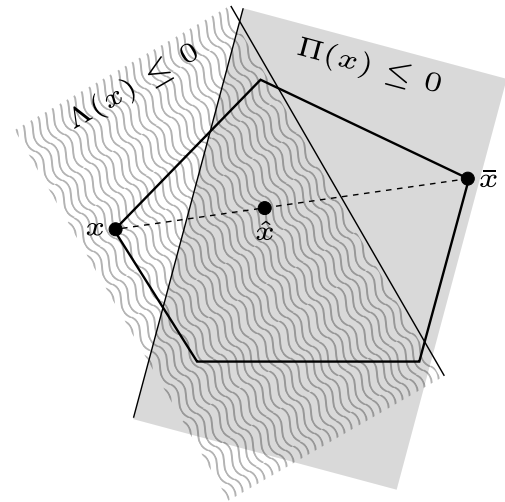

(a) For every $x \in P, \Lambda(x) \leq 0$ or $\Pi(x) \leq 0$. Condition 1 holds, $\bar{x}$ is a reference point.

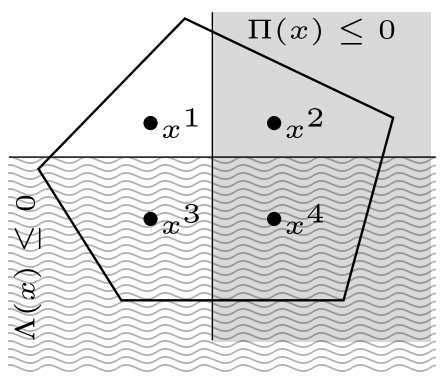

(b) None of the four conditions is satisfied. There is no reference point.

Figure 8.1: Example of polytope $P$, and two affine functions $\Lambda$ and $\Pi$.

Theorem 8.12. Let $k, \ell \in \mathbb{N}$. Let $\left(\lambda, \lambda_{0}\right) \in \mathbb{R}^{k \eta} \times \mathbb{R}$ and $\left(\pi, \pi_{0}\right) \in \mathbb{R}^{\ell \eta} \times \mathbb{R}$ be facet-defining inequalities of $\mathcal{P}_{k}(G)$ and $\mathcal{P}_{\ell}(G)$. For $x=(y, z) \in \mathbb{R}^{k \eta} \times \mathbb{R}^{\ell \eta}$, let $\Lambda(x)=\lambda y-\lambda_{0}$ and $\Pi(x)=\pi z-\pi_{0}$. If $\Lambda(x) \leq 0$ or $\Pi(x) \leq 0$, for every $x \in \mathcal{P}_{k+\ell}(G)$, then the following inequalities are facet-defining for $\mathcal{P}_{k+\ell}(G)$.

$$
\begin{aligned}
& \left(\lambda \bar{y}-\lambda_{0}\right)\left(\pi z-\pi_{0}\right)-\left(\pi \bar{z}-\pi_{0}\right)\left(\lambda y-\lambda_{0}\right) \leq 0, \\
& \left(\lambda \tilde{y}-\lambda_{0}\right)\left(\pi z-\pi_{0}\right)-\left(\pi \tilde{z}-\pi_{0}\right)\left(\lambda y-\lambda_{0}\right) \geq 0,
\end{aligned}
$$

where $\bar{x}=(\bar{y}, \bar{z})$ and $\tilde{x}=(\tilde{y}, \tilde{z})$ are reference points, in $\mathcal{P}_{k+\ell}(G)$, for $\Lambda$ with respect to $\Pi$ and for $\Pi$ with respect to $\Lambda$, respectively.

Proof. By Lemma 8.11, points $\bar{x}=(\bar{y}, \bar{z}) \in \mathbb{R}^{k \eta} \times \mathbb{R}^{\ell \eta}$ and $\tilde{x}=(\tilde{y}, \tilde{z}) \in \mathbb{R}^{k \eta} \times \mathbb{R}^{\ell \eta}$ do exist. By applying (8.1) with $\bar{x}$ and $\hat{x}$, we respectively get inequalities (8.3) and (8.4), which are therefore valid for $\mathcal{P}_{k+\ell}(G)$. To show that they define facets, we exhibit appropriate affinely independent points. Since $\left(\lambda, \lambda_{0}\right)$ is a facetdefining inequality of $\mathcal{P}_{k}(G)$, there exist $p$ affinely independent vectors $y^{1}, y^{2}, \ldots, y^{p}$ in $\mathcal{P}_{k}(G)$ satisfying $\left(\lambda, \lambda_{0}\right)$ at equality, where $p=k \eta$. Analogously, there exist $q$ affinely independent vectors $z^{1}, z^{2}, \ldots, z^{q}$ in $\mathcal{P}_{\ell}(G)$ satisfying $\left(\pi, \pi_{0}\right)$ at equality, where $q=\ell \eta$. One may easily verify that the following $(k+\ell) \eta-1$ vectors are affinely independent points belonging to $\mathcal{P}_{k+\ell}(G) \supseteq \mathcal{P}_{k}(G) \times \mathcal{P}_{\ell}(G)$ :

$$
\left(y^{1}, z^{1}\right),\left(y^{2}, z^{1}\right), \ldots,\left(y^{p}, z^{1}\right),\left(y^{1}, z^{2}\right),\left(y^{1}, z^{3}\right), \ldots,\left(y^{1}, z^{q}\right) .
$$

Moreover, they satisfy inequalities $\left(\lambda, \mathbf{0}, \lambda_{0}\right),\left(\mathbf{0}, \pi, \pi_{0}\right),(8.3)$ and (8.4) at equality. Now, consider $\bar{x}$. It also satisfies (8.3) at equality. However, by Definition 8.8, we have $\Lambda(\bar{x}) \neq 0$ or $\Pi(\bar{x}) \neq 0$, which means that $\bar{x}$ does not belong to the supporting hyperplane of $\left(\lambda, \mathbf{0}, \lambda_{0}\right)$ or $\left(\mathbf{0}, \pi, \pi_{0}\right)$. Therefore, the $(k+\ell) \eta-1$ presented vectors together with $\bar{x}$ are $(k+\ell) \eta$ affinely independent vectors in the face defined by (8.3), which is, consequently, a facet of $\mathcal{P}_{k+\ell}(G)$. The situation for $\tilde{x}$ and Inequality (8.4) is similar, thus leading to the conclusion that (8.4) is also facet-defining. 


\subsection{Facets from $\mathcal{P}_{1}\left(G \circ K_{k}\right)$ to $\mathcal{P}_{k}(G)$}

The lexicographic product of two graphs $G_{1}$ and $G_{2}$ is the graph $G_{1} \circ G_{2}$ that is obtained by replacing each vertex of $G_{1}$ by a copy of $G_{2}$ and also making two vertices in $G_{1} \circ G_{2}$ adjacent whenever the corresponding vertices in $G_{1}$ are adjacent. More formally, $V\left(G_{1} \circ G_{2}\right)=\left\{v_{w}: v \in V\left(G_{1}\right), w \in V\left(G_{2}\right)\right\}$ and $E\left(G_{1} \circ G_{2}\right)=\left\{v_{w} v_{z}: v \in V\left(G_{1}\right), w z \in E\left(G_{2}\right)\right\} \cup\left\{v_{w} u_{z}: v u \in E\left(G_{1}\right), w, z \in V\left(G_{2}\right)\right\}$. Let $K_{k}$ denote a clique with $k$ vertices. It is clear that $\chi_{k}(G)=\chi_{1}\left(G \circ K_{k}\right)$.

For each $v \in V(G)$ and $i \in[k]$, let $v_{i} \in V\left(G \circ K_{k}\right)$ denote the $i$-th copy of $v$ in $G \circ K_{k}$. Given the total order $\prec$ on $V(G)$, we define a total order $\prec^{\circ}$ on $V\left(G \circ K_{k}\right)$ as follows:

- $v_{i} \prec^{\circ} v_{j}$, for every $v \in V(G)$, and $i, j \in[k]$ such that $i<j$; and

- $u_{i} \prec^{\circ} v_{j}$, for every $u, v \in V(G)$ such that $u \prec v$, and for every $i, j \in[k]$.

Thus, if $u$ is not a neighbor of $v$ in $G$ and $u \prec v$, then $u_{i}$ is not a neighbor of $v_{j}$ in $G \circ K_{k}$ and $u_{i} \prec^{\circ} v_{j}$, for all $i, j \in[k]$. Note that, if $v$ is a source in $G$, then $v_{1}, v_{2}, \ldots, v_{k}$ are sources in $G \circ K_{k}$.

We observe that $\mathcal{P}_{k}(G) \subseteq \mathbb{R}^{p}$ whereas $\mathcal{P}_{1}\left(G \circ K_{k}\right) \subseteq \mathbb{R}^{q}$, where $p=k\left(n^{\prime}+\bar{m}\right)$ and $q=k\left(n^{\prime}+k \bar{m}\right)$. As $k$ increases, the difference $q-p$ increases (if $G$ is not a complete graph). This is a reason to explore formulation $\mathcal{R}_{k}(G)$ instead of simply using $\mathcal{R}_{1}\left(G \circ K_{k}\right)$. However, we profit from the known results for $\mathcal{P}_{1}\left(G \circ K_{k}\right)$ to describe $\mathcal{P}_{k}(G)$. Next, we show that some facets of the former can be projected into facets of the latter.

Theorem 8.13. Let $k \in \mathbb{N}$ and let $\left(\lambda, \lambda_{0}\right) \in \mathbb{R}^{q} \times \mathbb{R}$ be a facet-defining inequality of $\mathcal{P}_{1}\left(G \circ K_{k}\right)$. If, for every $v \in V^{\prime}, u \in \bar{N}^{-}(v)$ and $i \in[k]$, there exists $\lambda_{u_{i} v}$ such that $\lambda_{u_{i} v}=\lambda_{u_{i} v_{j}}$ for all $j \in[k]$, then $\left(\hat{\lambda}, \lambda_{0}\right) \in \mathbb{R}^{p} \times \mathbb{R}$ is a facet-defining inequality of $\mathcal{P}_{k}(G)$, where $\hat{\lambda}_{v v}^{i}=\lambda_{v_{i} v_{i}}$ and $\hat{\lambda}_{u v}^{i}=\lambda_{u_{i} v}$, for all $v \in V^{\prime}$, $u \in \bar{N}^{-}(v)$ and $i \in[k]$.

Proof. Let $\left(\lambda, \lambda_{0}\right) \in \mathbb{R}^{q} \times \mathbb{R}$ be a facet-defining inequality of $\mathcal{P}_{1}\left(G \circ K_{k}\right)$ such that $\lambda_{u_{i} v_{1}}=\lambda_{u_{i} v_{2}}=\ldots=$ $\lambda_{u_{i} v_{k}}$ for all $v \in V^{\prime}, u \in \bar{N}^{-}(v)$ and $i \in[k]$. We define a function $f: \mathcal{P}_{1}\left(G \circ K_{k}\right) \rightarrow \mathcal{P}_{k}(G)$ as follows. For every $x \in \mathcal{P}_{1}\left(G \circ K_{k}\right), y=f(x)$ is such that $y_{u u}^{i}=x_{u_{i} u_{i}}$ for all $u \in V^{\prime}, i \in[k]$, and $y_{u v}^{i}=\sum_{j=1}^{k} x_{u_{i} v_{j}}$ for all $v \in V^{\prime}, u \in \bar{N}^{-}(v), i \in[k]$. Note that $f$ may be described by a $p \times q$ transformation matrix $A$ (that is, $f(x)=A x)$ of the form

$$
A=[\overbrace{e^{1} \ldots e^{k n^{\prime}}}^{k n^{\prime}} \overbrace{e^{k n^{\prime}+1} \ldots e^{k n^{\prime}+1}}^{k} \ldots \overbrace{e^{k n^{\prime}+k \bar{m}} \ldots e^{k n^{\prime}+k \bar{m}}}^{k}] .
$$

Clearly, we have $\operatorname{rank}(A)=p$. Moreover, note that $A x \in \mathcal{P}_{k}(G)$ for every $x \in \mathcal{P}_{1}\left(G \circ K_{k}\right)$.

Claim 8.13.1. $f: \mathcal{P}_{1}\left(G \circ K_{k}\right) \rightarrow \mathcal{P}_{k}(G)$ is an onto function.

Proof. In order to show that $f$ is an onto function, given a vector $\hat{y}$ in $Y=\mathcal{P}_{k}(G) \cap \mathbb{B}^{p}$, we exhibit a vector $\hat{x}$ in $X=\mathcal{P}_{1}\left(G \circ K_{k}\right) \cap \mathbb{B}^{q}$ such that $A \hat{x}=\hat{y}$. The result then follows as $\mathcal{P}_{k}(G)=\operatorname{conv}(Y)$ and $\mathcal{P}_{1}\left(G \circ K_{k}\right)=\operatorname{conv}(X)$. Consider a vertex $v \in V^{\prime}$. Let $R:=\left\{i: \hat{y}_{v v}^{i}=1\right\} \subseteq[k]$, and let $\pi:[r] \rightarrow[k]$ be a function such that $\langle\pi(1), \pi(2), \ldots, \pi(r)\rangle$ is a non-decreasing sequence of the elements in $[k] \backslash R$, where $r=k-|R|$. Let $Q:=\left\{(u, i) \in \bar{N}^{-}(v) \times[k]: \hat{y}_{u v}^{i}=1\right\}$, and $\left\langle t_{1}, t_{2}, \ldots, t_{s}\right\rangle$ be the elements of $Q$ in lexicographic order, where $s=|Q|$. Note that $s \geq r$ since $\hat{y}$ defines a $k$-fold coloring of $G$.

We now set the non-zero components of $\hat{x}$ related to the colors represented by $v$ and to the colors of vertices in $\bar{N}^{-}(v)$ that represents $v$ in the following way:

- $\hat{x}_{v_{i} v_{i}}:=1$ for each $i \in R$, 
- $\hat{x}_{u_{i} v_{\pi(\ell)}}:=1$ for each $\ell \in\{1,2, \ldots, r\}$, where $t_{\ell}=(u, i) \in Q$, and

- if $s>r$, then we define $\hat{x}_{u_{i} v_{1}}=1$ for all $\ell \in\{r+1, \ldots, s\}$, where $t_{\ell}=(u, i) \in Q$.

By the above construction, it is not hard to verify that $A \hat{x}=\hat{y}$. Moreover, since the coloring of $G$ induced by $\hat{y}$ ensures that each vertex has at least $k$ colors, we obtain that each copy of $v$ in $G \circ K_{k}$ has at least one color. Therefore, the vector $\hat{x}$ belongs to the polytope $\mathcal{P}_{1}\left(G \circ K_{k}\right)$.

Let $\left(\hat{\lambda}, \hat{\lambda}_{0}\right) \in \mathbb{R}^{p} \times \mathbb{R}$ be an inequality such that $\hat{\lambda}_{u u}^{i}=\lambda_{u_{i} u_{i}}$ for all $u \in V^{\prime}, i \in[k], \hat{\lambda}_{u v}^{i}=\lambda_{u_{i} v_{1}}=$ $\lambda_{u_{i} v_{2}}=\ldots=\lambda_{u_{i} v_{k}}$ for all $v \in V^{\prime}, u \in \bar{N}^{-}(v), i \in[k]$, and $\hat{\lambda}_{0}=\lambda_{0}$. Note that $\hat{\lambda}^{T} A=\lambda^{T}$. Since $\left(\lambda, \lambda_{0}\right)$ is a valid inequality of $\mathcal{P}_{1}\left(G \circ K_{k}\right)$, we have, for every $y \in \mathcal{P}_{k}(G), \hat{\lambda}^{T} y=\hat{\lambda}^{T}(A x)=\left(\hat{\lambda}^{T} A\right) x=\lambda^{T} x \leq \lambda_{0}=\hat{\lambda}_{0}$, where $x$ is the vector in $\mathcal{P}\left(G \circ K_{k}\right)$ defined in Claim 8.13.1. Therefore, the inequality $\left(\hat{\lambda}, \hat{\lambda}_{0}\right)$ is valid for $\mathcal{P}_{k}(G)$.

Let $F:=\left\{x \in \mathcal{P}_{1}\left(G \circ K_{k}\right): \lambda^{T} x=\lambda_{0}\right\}$ and $\hat{F}:=\left\{y \in \mathcal{P}_{k}(G): \hat{\lambda}^{T} y=\hat{\lambda}_{0}\right\}$ be faces of $\mathcal{P}_{1}\left(G \circ K_{k}\right)$ and $\mathcal{P}_{k}(G)$, respectively. Similar arguments to those above show that $A x \in \hat{F}$, for every $x \in F$. This remark is usefull in the rest of the proof.

Claim 8.13.2. If $0 \in$ affine $(F)$, then $\mathbf{0} \in$ affine $(\hat{F})$.

Proof. If $\mathbf{0} \in \operatorname{affine}(F)$, then there exist vectors $x_{1}, \ldots, x_{\ell} \in F$ and scalars $\alpha_{1}, \ldots, \alpha_{\ell} \in \mathbb{R}$ such that $\sum_{i=1}^{\ell} \alpha_{i} x_{i}=\mathbf{0}$ and $\sum_{i=1}^{\ell} \alpha_{i}=1$, where $\ell \in \mathbb{N}$. Thus, $\sum_{i=1}^{\ell} \alpha_{i} A x_{i}=\sum_{i=1}^{\ell} \alpha_{i} y_{i}=\mathbf{0}$ with $y_{i}=A x_{i} \in \hat{F}$ for all $i \in[\ell]$. Therefore, we obtain $\mathbf{0} \in \operatorname{affine}(\hat{F})$.

Finally, to show that $\operatorname{dim}(\hat{F})=\operatorname{dim}\left(\mathcal{P}_{k}(G)\right)-1$, we consider two cases. First, suppose that $\mathbf{0} \in$ affine $(F)$. Since $F$ is a facet of $\mathcal{P}_{1}\left(G \circ K_{k}\right)$, there are $q-1$ linearly independent vectors in $F$, say $x_{1}, x_{2}, \ldots, x_{q-1}$. Consider the matrix $B=\left[x_{1} x_{2} \ldots x_{q-1}\right]$ with $\operatorname{rank}(B)=q-1$. The Sylvester's rank inequality yields that $\operatorname{rank}(A B) \geq p-1$. Thus, the set $H:=\left\{A x_{1}, A x_{2}, \ldots, A x_{q-1}\right\}$ has at least $p-1$ linearly independent vectors. Since $H \subseteq \hat{F}$ and $\mathbf{0} \in \operatorname{affine}(\hat{F})$ by Claim 8.13 .2 , we can conclude that $\operatorname{dim}(\hat{F})=\operatorname{rank}(\hat{F}) \geq$ $p-1=\operatorname{dim}\left(\mathcal{P}_{k}(G)\right)-1$. Now, suppose that $\mathbf{0} \notin$ affine $(F)$. Similarly to the first case, we get $q$ (instead of $q-1)$ linearly independent vectors in $F$. The corresponding set $H$ contains now $p$ linearly independent vectors of $\hat{F}$, which again implies $\operatorname{dim}(\hat{F}) \geq \operatorname{rank}(\hat{F})-1 \geq p-1$. In both cases, we conclude that $\hat{F}$ is a facet of $\mathcal{P}_{k}(G)$.

\subsection{Facets from stable set polytopes to $\mathcal{P}_{k}(G)$}

A stable set of a graph $G$ is a subset of pairwise non-adjacent vertices. The stable set polytope of $G$ is defined as

$$
\operatorname{STAB}(G)=\operatorname{conv}\left\{x \in \mathbb{B}^{|V(G)|}: x_{u}+x_{v} \leq 1, \text { for all } u v \in E(G)\right\}
$$

It is known that $\operatorname{staB}(G)$ is full-dimensional. Furthermore, there are many results about the facial structure of $\operatorname{stab}(G)[23,24,37,40,41,63,76]$. Given a subset of vertices $H \subseteq V(G)$, let $G[H]$ be the subgraph of $G$ induced by $H$. For simplicity, let $G^{+}(u)$ denote the subgraph $G\left[\bar{N}^{+}(u)\right]$.

We next show that, for all $u \in V(G)$, the facets of $\operatorname{staB}\left(G^{+}(u)\right)$ are inherited by $\mathcal{P}_{k}(G)$. This provides a relation between the facet-defining inequalities of the stable set polytope and the external inequalities that define facets of $\mathcal{P}_{1}(G)$ [16]. Additionally, it implies that these inequalities derived in [16] are also facet-defining for $\mathcal{P}_{k}(G)$. 
Theorem 8.14. Let $k \in \mathbb{N}$ and $u \in V(G)$. If the inequality $\sum_{v \in \bar{N}^{+}(u)} \lambda_{v} x_{v} \leq \lambda_{0}$ is facet-defining for $\operatorname{STAB}\left(G^{+}(u)\right)$, then, for every $i \in[k]$, the inequality $\sum_{v \in \bar{N}^{+}(u)} \lambda_{v} x_{u v}^{i} \leq \lambda_{0} y_{u}^{i}$ is facet-defining for $\mathcal{P}_{k}(G)$, where $y_{u}^{i}=1$ if $u \in M$ (i.e., $u$ is a source), and $y_{u}^{i}=x_{u u}^{i}$ otherwise.

Proof. First, we prove that the inequality $\sum_{v \in \bar{N}^{+}(u)} \lambda_{v} x_{u v}^{i} \leq \lambda_{0} y_{u}^{i}$ is valid for $\mathcal{P}_{k}(G)$, where $i \in[k]$. Let $x \in \mathcal{P}_{k}(G)$. If $y_{u}^{i}=x_{u u}^{i}=0$, it is valid due to constraints (7.4) of $\mathcal{R}_{k}(G)$. Otherwise, $y_{u}^{i}=1$, and so we obtain that $u$ represents the stable set $S=\left\{v \in \bar{N}^{+}(u): x_{u v}^{i}=1\right\}$. Since $\sum_{v \in \bar{N}^{+}(u)} \lambda_{v} x_{v} \leq \lambda_{0}$ is valid for $\operatorname{STAB}\left(G^{+}(u)\right)$, we obtain that $\sum_{v \in S^{\prime}} \lambda_{v} \leq \lambda_{0}$ for every stable set $S^{\prime} \subseteq \bar{N}^{+}(u)$. Therefore, we conclude that $\sum_{v \in \bar{N}^{+}(u)} \lambda_{v} x_{u v}^{i}=\sum_{v \in S} \lambda_{v} \leq \lambda_{0}$ is valid for $\mathcal{P}_{k}(G)$.

Now, we prove that it is facet-defining. Let $p=\left|\bar{N}^{+}(u)\right|$ and let $\left\{x^{1}, x^{2}, \ldots, x^{p}\right\}$ be a set of affinely independent vectors in the given facet of $\operatorname{staB}\left(G^{+}(u)\right)$. Note that, for each $\ell \in[p], x^{\ell}$ is an incidence vector of a stable set of $G^{+}(u)$. We now show $n^{\prime}+\bar{m}$ points in $\mathcal{P}_{1}(G)$, each of them is of the form $X=$ $\left(x^{v v}, x^{u v}, x^{v w}\right)$ which respectively group the entries $x_{v v}$ for all $v \in V^{\prime}, x_{u v}$ for all $v \in \bar{N}^{+}(u)$, and $x_{v w}$ for all $v \in V(G) \backslash\{u\}, w \in \bar{N}^{+}(v)$. For every $\ell \in[p]$, let $X^{\ell}=\left(e^{V^{\prime}}, x^{\ell}, \mathbf{0}\right)$. Observe that $X^{\ell}$ describes the 1-fold coloring whose color classes are: $\{z\}$ for every $z \in V$, and the stable set of $G^{+}(u)$ induced by $x^{\ell}$. For each $v \in V(G) \backslash\{u\}, w \in \bar{N}^{+}(v)$, define $X^{v w}=\left(e^{V^{\prime}}, x^{1}, e^{v w}\right)$. Finally, for each $v \in V^{\prime}$, define $X^{v}=\left(e^{V^{\prime}}-e^{v}, x^{1}, e^{r_{v} v}\right)$ if $v \neq u$, and $X^{v}=\left(e^{V^{\prime}}-e^{v}, \mathbf{0}, e^{r_{v} v}\right)$ if $v=u$. One may easily check that these $n^{\prime}+\bar{m}$ vectors describe 1 -fold colorings of $G$. Moreover, it readily follows that they are affinely independent, provided that $\left\{x^{1}, x^{2}, \ldots, x^{p}\right\}$ is an affinely independent set.

Let $\ell \in[p], v \in V(G)$ and $w \in \bar{N}^{+}(v)$. Observe that $\sum_{z \in \bar{N}^{+}(u)} \lambda_{z} x_{z}^{\ell}=\lambda_{0}$. Thus, if $v \neq u$, then

$$
\sum_{z \in \bar{N}^{+}(u)} \lambda_{z} X_{u z}^{\ell}=\sum_{z \in \bar{N}^{+}(u)} \lambda_{z} X_{u z}^{v w}=\sum_{z \in \bar{N}^{+}(u)} \lambda_{z} X_{u z}^{v}=\sum_{z \in \bar{N}^{+}(u)} \lambda_{z} x_{z}^{1}=\lambda_{0} .
$$

In addition,

$$
\sum_{z \in \bar{N}^{+}(u)} \lambda_{z} X_{u z}^{u}=0
$$

Since $y_{u}^{i}=0$ only holds for vector $X^{u}$, all the enumerated vectors satisfy $\sum_{v \in \bar{N}^{+}(u)} \lambda_{v} x_{u v}^{i} \leq \lambda_{0} y_{u}^{i}$ at equality. Therefore, it defines a facet of $\mathcal{P}_{1}(G)$. The extension to $k \geq 2$ follows by Corollary 8.2 and Lemma 7.3.

Theorem 8.14 encompasses all the external inequalities described in [16] for $k=1$ and extends them to $k \geq 2$. These are inequalities of the form

$$
\sum_{v \in H} \frac{1}{\alpha_{v}} x_{u v}^{i} \leq y_{u}^{i} \quad \text { for } u \in V(G) \text { and } H \subseteq \bar{N}^{+}(u),
$$

where $\alpha_{v}$ is the maximum size of a stable set of $G[H]$ containing $v$. They are facet-defining under certain conditions on $H$, for instance if $H$ is a maximal clique in $G^{+}(u)$. In this case, the corresponding above inequality dominates (7.3) and (7.4) of $\mathcal{R}_{k}(G)$.

At this point, one could think of using Theorems 8.14 and 8.13 together to derive facets of $\mathcal{P}_{k}(G)$. Precisely, one could convert facet-defining inequalities of stable set polytopes of subgraphs of $G \circ K_{k}$ into facet-defining inequalities of $\mathcal{P}_{1}\left(G \circ K_{k}\right)$ by means of Theorem 8.14 and then attempt to convert it to a facet-defining inequality of $\mathcal{P}_{k}(G)$ by Theorem 8.13. As the next result shows, the inequalities obtained using this strategy can be obtained directly from stable set polytopes of subgraphs of $G$.

Let $G_{k}$ denote the graph $G \circ K_{k}$. Note that the positive anti-neighborhood of $u_{i}$ in $G_{k}$, taking into account the order $\prec^{\circ}$, is $\left\{v_{j}: v \in \bar{N}^{+}(u), j \in[k]\right\}$. This remark is repeatedly used in the proof of the 
next corollary.

Corollary 8.15. Let $k \in \mathbb{N}, i \in[k]$ and $u \in V(G)$. If the inequality

$$
\sum_{j \in[k]} \sum_{v \in \bar{N}^{+}(u)} \lambda_{v_{j}} x_{v_{j}} \leq \lambda_{0}
$$

is different from $x_{v_{j}} \geq 0$ for every $v \in V(G)$ and $j \in[k]$, and is facet-defining for $\operatorname{STAB}\left(G_{k}^{+}\left(u_{i}\right)\right)$, then

(a) for each $v \in \bar{N}^{+}(u)$, there is $\hat{\lambda}_{v}$ such that $\lambda_{v_{j}}=\hat{\lambda}_{v}$, for all $j \in[k]$; and

(b) $\sum_{v \in \bar{N}^{+}(u)} \hat{\lambda}_{v} x_{u v}^{i} \leq \lambda_{0} y_{u}^{i}$ is facet-defining for $\mathcal{P}_{k}(G)$, where $y_{u}^{i}=1$ if $u \in M$, and $y_{u}^{i}=x_{u u}^{i}$ otherwise.

Proof. To prove item (a), let $j, \ell \in[k], j \neq \ell$. Let $F$ be the facet of $\operatorname{STAB}\left(G_{k}^{+}\left(u_{i}\right)\right)$ defined by (8.5). Since $F$ is not the face induced by $x_{v_{j}}=0$, there is $\bar{x} \in F$ such that $\bar{x}_{v_{j}}=1$, and so $\bar{x}_{v_{\ell}}=0$ (because $\left.v_{j} v_{\ell} \in E\left(G_{k}^{+}\left(u_{i}\right)\right)\right)$, that is, the stable set $\bar{S}$ defined by $\bar{x}$ contains $v_{j}$ but not $v_{\ell}$. Replacing $v_{j}$ by $v_{\ell}$, we obtain another stable set $\hat{S}=\bar{S} \cup\left\{v_{\ell}\right\} \backslash\left\{v_{j}\right\}$ of $G_{k}^{+}\left(u_{i}\right)$ whose characteristic vector $\hat{x}$ must satisfy Inequality (8.5). Regarding the difference between $\hat{x}$ and $\bar{x}$, and the fact that $\bar{x}$ satisfies that inequality at equality, we conclude that $\lambda_{v_{\ell}} \leq \lambda_{v_{j}}$. Interchanging the roles of $j$ and $\ell$ in the previous argument, we obtain the converse inequality, and so $\lambda_{v_{\ell}}=\lambda_{v_{j}}$.

By Theorem 8.14, we conclude that $\sum_{j \in[k]} \sum_{v \in \bar{N}^{+}(u)} \hat{\lambda}_{v} x_{u_{i} v_{j}} \leq \lambda_{0} y_{u_{i}}$ defines a facet of $\mathcal{P}_{1}\left(G_{k}\right)$, where $y_{u_{i}}=1$ if $u \in M$, and $y_{u_{i}}=x_{u_{i} u_{i}}$ otherwise. Therefore, item (b) follows by Theorem 8.13.

Observe that using the relation $x_{v}=\sum_{j \in[k]} x_{v_{j}}$, for $v \in V(G)$, to map a point in $\operatorname{STAB}\left(G_{k}\right)$ to a point in $\operatorname{StAB}(G)$, we can proceed similarly to the proof of Theorem 8.13 to show that $\sum_{v \in \bar{N}^{+}(u)} \hat{\lambda}_{v} x_{v} \leq \lambda_{0}$ defines a facet of $\operatorname{staB}\left(G^{+}(u)\right)$. Thus, the facets of $\mathcal{P}_{k}(G)$ obtained from $\operatorname{STAB}\left(G_{k}^{+}\left(u_{i}\right)\right)$ by Corollary 8.15 are included in those obtained directly from $\operatorname{staB}\left(G^{+}(u)\right)$ by Theorem 8.14. 


\section{Chapter 9}

\section{Facets induced by critical subgraphs}

In this chapter, we derive a class of facet-defining inequalities associated with induced subgraphs, namely webs and antiwebs (see Sections 9.2 and 9.3). We also characterize, in Section 9.1, a criticality property required by these graphs to induce facets of $\mathcal{P}_{k}(G)$.

\subsection{Critical graphs}

For integers $p \geq 2$ and $n \geq 2 p$, Trotter [76] defined the web $W_{p}^{n}$ as the graph with vertex set $\left\{v_{0}, v_{1}, \ldots, v_{n-1}\right\}$ and edge set $\left\{v_{i} v_{j}: p \leq|i-j| \leq n-p\right\}$ (see Figure 9.1(a)). The antiweb $\bar{W}_{p}^{n}$ is defined as the complement of $W_{p}^{n}$ (see Figure 9.1(b)). We observe that these definitions are interchanged in some references (see [66, 78], for instance).

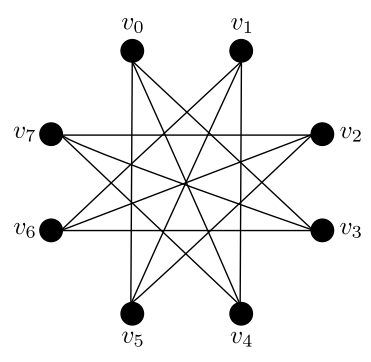

(a) $W_{3}^{8}$

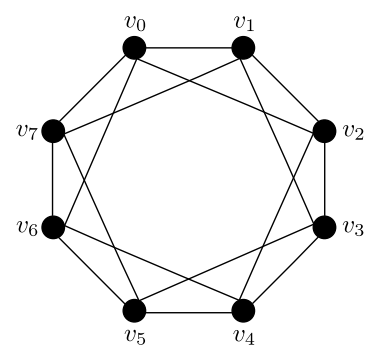

(b) $\bar{W}_{3}^{8}$

Figure 9.1: Example of a web and an antiweb.

Given two distinct vertices $v_{i}, v_{j}$ belonging to $V\left(W_{p}^{n}\right)$ (or to $V\left(\bar{W}_{p}^{n}\right)$ ), we say that $v_{i}$ is on the right of $v_{j}$ (or equivalently that $v_{j}$ is on the left of $\left.v_{i}\right)$ if $(i-j) \bmod n \leq(j-i) \bmod n$, that is, when going from $v_{j}$ to $v_{i}$, the "clockwise" distance is at most the "anti-clockwise" distance. In Figure 9.1, $v_{1}, v_{2}, v_{3}, v_{4}$ are on the right of $v_{0}$ whereas $v_{5}, v_{6}, v_{7}$ are on the left of $v_{0}$.

Webs and antiwebs form subclasses of circulant graphs that play an important role in the context of stable sets and vertex coloring problems $[23,24,37,40,41,63,65,66,78]$. They include odd holes $\left(W_{\ell}^{2 \ell+1}\right)$ and odd anti-holes $\left(W_{2}^{2 \ell+1}\right)$. In [18], it was proved that $\chi_{k}(G)=\left\lceil\frac{n k}{\alpha(G)}\right\rceil$ if $G=W_{p}^{n}$ or $G=\bar{W}_{p}^{n}$, where $\alpha(G)$ denotes the size of a maximum stable set of $G$. By its turn, it is known that $\alpha\left(W_{p}^{n}\right)=p$ and $\alpha\left(\bar{W}_{p}^{n}\right)=\left\lfloor\frac{n}{p}\right\rfloor[76]$.

Facet-defining inequalities of $\mathcal{P}_{1}(G)$ based on odd holes and odd anti-holes are presented in [16]. More 
recently, other webs and antiwebs are considered to obtain facets of an 1-fold coloring polytope associated with a formulation similar to $\mathcal{R}_{1}(G)$ [65]. Next, we generalize these results for $\mathcal{P}_{k}(G)$. To identify webs and antiwebs inducing facets of $\mathcal{P}_{k}(G)$, we introduce some concepts.

For every $k \in \mathbb{N}$, a graph $G$ is $\chi_{k^{-}}$critical if $\chi_{k}(G-v)<\chi_{k}(G)$, for each vertex $v \in V(G)$. The $\chi_{k^{-}}$ critical webs and antiwebs are characterized in [18]. Here, we introduce a stronger criticality condition. We say that $G$ is strongly $\chi_{k}$-critical if, for every $v \in V(G), G$ admits an optimal $k$-fold coloring in which the singleton set $\{v\}$ is a color class. Note that the two concepts are equivalent for $k=1$.

In this section, we characterize strongly $\chi_{k}$-critical webs and antiwebs and show that they induce facets of $\mathcal{P}_{k}(G)$. We observe that the webs considered in [65], which include odd holes and odd anti-holes, are all (strongly) $\chi_{1}$-critical.

Theorem 9.1. For every $k \in \mathbb{N}, G$ is a strongly $\chi_{k}$-critical web $W_{p}^{n}$ or antiweb $\bar{W}_{p}^{n}$ if, and only if, $\frac{k n-1}{\alpha(G)} \in \mathbb{Z}$.

Proof. Firstly, suppose that $G$ is a strongly $\chi_{k}$-critical web $W_{p}^{n}$ or antiweb $\bar{W}_{p}^{n}$. In any optimal $k$-fold coloring of $G$, we have $\chi_{k}(G)=\left\lceil\frac{n k}{\alpha(G)}\right\rceil$ color classes, each of them with at most $\alpha(G)$ vertices. One can easily check that, if one color class is a singleton, the other ones must have exactly $\alpha(G)$ vertices. Therefore, $\left(\left[\frac{n k}{\alpha(G)}\right]-1\right) \alpha(G)=n k-1$, and thus $\frac{n k-1}{\alpha(G)} \in \mathbb{N}$.

Later on, in Lemmas 9.4 and 9.9, we shall prove that this condition is also sufficient.

The subgraph-induced inequalities we consider are presented in the following theorem. For $k=1$, they are the internal inequalities defined in [16], where they are shown to define facets of $\mathcal{P}_{1}(G)$ when $H$ induces an odd hole or an odd-antihole. Next, we generalize this result for an arbitrary $k \in \mathbb{N}$ and extend it to strongly $\chi_{k}$-critical webs and antiwebs.

Theorem 9.2. Let $k \in \mathbb{N}, H \subseteq V(G)$ and $M_{H}$ be the set of sources in the suborder of $\prec$ restricted to $H$, that is, $M_{H}=\left\{v \in H: \bar{N}^{-}(v) \cap H=\emptyset\right\}$. If $G[H]$ is a strongly $\chi_{k}$-critical web or antiweb, then the following inequality is facet-defining for $\mathcal{P}_{k}(G)$.

$$
\sum_{v \in H \backslash M_{H}} \sum_{u \in\left(\bar{N}^{-}(v) \backslash H\right) \cup\{v\}} \sum_{i=1}^{k} x_{u v}^{i} \geq \chi_{k}(G[H])-k\left|M_{H}\right| .
$$

Proof. First, let us prove that Inequality (9.1) is valid for $\mathcal{P}_{k}(G)$. In any $k$-fold coloring of $G$, each color appearing in $H$ that does not occur in $M_{H}$ adds at least one to the left-hand side of Inequality (9.1). Since $M_{H}$ induces a clique in $G[H]$ and at least $\chi_{k}(G[H])$ are necessary to color this graph, the inequality is valid.

Later on, we prove facetness of Inequality (9.1) separately for webs and antiwebs in Lemmas 9.5 and 9.13 , respectively.

In the remaining, we need some additional notation. Let $G$ be a graph and $H \subseteq V(G)$. Let $C$ be a $k$-fold coloring of $G$, where each vertex receives exactly $k$ colors. We say that $C$ is a $c r i t i c a l k$-fold coloring (with respect to $H$ ) if it optimally colors $G[H]$ and every vertex outside $H$ does not share any of its $k$ colors with other vertex. More precisely, describing $C$ as a sequence $C=\left\langle S_{1}, \ldots, S_{t}\right\rangle$ of $t \geq \chi_{k}(G)$ stable sets, $C$ is critical if $\left\{S_{1}, S_{2}, \ldots, S_{\chi_{k}(G[H])}\right\}$ defines an optimal $k$-fold coloring of $G[H]$ and, for each $v \in V(G) \backslash H$, there exist distinct integers $c_{1}, c_{2}, \ldots, c_{k}$ such that $\chi_{k}(G[H])<c_{i} \leq t$ for all $i \in[k]$, and $S_{c_{1}}=S_{c_{2}}=\cdots=S_{c_{k}}=\{v\}$. For each $v \in V(G)$, let $S^{v}[C]$ denote the subset of $k$ stable sets in $C$ that contain $v$. We prefer the notation $S^{v}$ when $C$ is clear in the context and use $S^{v}=\left\langle S_{1}^{v}, S_{2}^{v}, \ldots, S_{k}^{v}\right\rangle$. 
Note that, if $G[H]$ is strongly $\chi_{k}$-critical, then, for each $v \in V(G)$ and $i \in[k]$, there is a critical $k$-fold coloring of $G$ such that $S_{i}^{v}=\{v\}$. Let $C^{v, i}$ denote such a critical $k$-fold coloring.

Recall that $M=\left\{v: \bar{N}^{-}(v)=\emptyset\right\}$ is the set of source vertices in the ordering $\prec$ of $V(G)$, and that $V^{\prime}=V(G) \backslash M$ is the set of non-minimal vertices. Moreover, recall that $n^{\prime}=\left|V^{\prime}\right|$ and that $\bar{m}$ is the number of edges of $\bar{G}$, the complement of $G$. For every $v \in V^{\prime}, u \in \bar{N}^{-}[v]$ and $i \in[k]$, the vector $e_{u v}^{i} \in \mathbb{B}^{k\left(n^{\prime}+\bar{m}\right)}$ is a binary vector in which the entry indexed by $u v$ and $i$ is the only non-zero entry. For every critical $k$-fold coloring $C$, we define its incidence vector $X(C) \in \mathbb{B}^{k\left(n^{\prime}+\bar{m}\right)}$, indexed by $v \in V^{\prime}$, $u \in \bar{N}^{-}[v]$ and $i \in[k]$, such that

$$
X(C)_{u v}^{i}=\left\{\begin{array}{rr}
1 & \text { if } u \text { represents } S_{i}^{u} \text { and } v \in S_{i}^{u} \\
0 & \text { otherwise }
\end{array}\right.
$$

Observe that, if we transform $C$ into a new $k$-fold coloring $C^{\prime}$ (non-necessarily critical), we can add (or subtract) unit vectors $e_{u v}^{i}$ to turn $X(C)$ into a point belonging to $P_{k}(G)$ that describes $C^{\prime}$. For instance, if $C^{\prime}$ is obtained from $C$ by creating a new color class $\{u\}$, where $u$ is not the representative of $S_{i}^{u}[C]$, then $C^{\prime}$ can be described by $X(C)+e_{u u}^{i}$.

Remark 9.3. If $C$ is a critical $k$-fold coloring with respect to $H$, then $X(C)$ belongs to the face of $\mathcal{P}_{k}(G)$ defined by Inequality (9.1).

Henceforth, assume that the vertices of web and antiwebs graphs are labeled in clockwise order (see Figure 9.1, for instance). Let $\oplus$ stands for addition modulus $n$, that is, $i \oplus j=(i+j) \bmod n$, for $i, j \in \mathbb{Z}$. The rest of this chapter is devoted to show sufficiency for the condition stated in Theorem 9.1 and facetness for Inequality (9.1). We prove them for webs in Section 9.2, and for antiwebs in Section 9.3.

\subsection{Web facets}

In [18], it was shown that the following sets are maximum stable sets of $W_{p}^{n}$ :

$$
I_{i}=\left\langle v_{i \oplus 0}, v_{i \oplus 1}, \ldots, v_{i \oplus(p-1)}\right\rangle \quad \text { for all } i \in \mathbb{N} \cup\{0\} .
$$

In this section, we use these stable sets to determine critical $k$-fold colorings of $G$ with respect to $H$ when $G[H]$ is a strongly $\chi_{k}$-critical web. We start characterizing webs having such a property.

Lemma 9.4. For every $k \in \mathbb{N}$, if $\frac{n k-1}{p} \in \mathbb{Z}$, then $W_{p}^{n}$ is strongly $\chi_{k}$-critical.

Proof. By the symmetry of $W_{p}^{n}$, it suffices to show an optimal $k$-fold coloring of $W_{p}^{n}$ where $\left\{v_{n-1}\right\}$ is a color class. Let $x=\frac{n k-1}{p} \in \mathbb{Z}$. By traversing the sequence $v_{0}, v_{1}, \ldots v_{n-1}$ of vertices of $W_{p}^{n}$ in a circular way, form $x$ subsets of $p$ vertices each, that is, $I_{0}, I_{p}, I_{2 p} \ldots, I_{(x-1) p}$. Note that each of these subsets defines a stable set. Moreover, they cover $k$ times each of the first $n-1$ vertices, namely $v_{0}, v_{1}, \ldots, v_{n-2}$, and $k-1$ times vertex $v_{n-1}$. Using this last vertex to form an additional color class, we obtain a $k$-fold coloring with $x+1=\frac{n k-1}{p}+1=\left\lceil\frac{n k}{p}\right\rceil$ colors, which is therefore optimal [18]. Hence, $W_{p}^{n}$ is strongly $\chi_{k}$-critical.

By the above proof, one can readily see that, if $G$ contains a strongly $\chi_{k^{-}}$critical web $W_{p}^{n}$ as subgraph, then, for every $i \in\{0,1, \ldots, n-1\}$, we can find a critical $k$-fold coloring $C^{v_{i}, 1}=\left\langle S_{1}, S_{2}, \ldots, S_{t}\right\rangle$ of $G$ in which $S_{1}=\left\{v_{i}\right\}$ and, alternatively, $S_{2}=I_{i \oplus 1}$ or $S_{2}=I_{i \oplus(-p)}$. In other words, if $v_{i} v_{j} \notin E\left(W_{p}^{n}\right)$, we can assume that $v_{j} \in S_{2}$ and that the $p+1$ vertices in $S_{1} \cup S_{2}$ appear consecutively in $W_{p}^{n}$. Such colorings are repeatedly used to prove facetness. 
Lemma 9.5. Let $k \in \mathbb{N}, H \subseteq V(G)$ and $M_{H}$ be the set of sources in the suborder of $\prec$ restricted to $H$. If $G[H]$ is a strongly $\chi_{k}$-critical web, then Inequality (9.1) induces a facet of $\mathcal{P}_{k}(G)$.

Proof. Consider a face $F=\left\{x \in P_{k}(G): \lambda^{T} x=\lambda_{0}\right\}$ of $P_{k}(G)$ such that $\hat{F}=\left\{x \in P_{k}(G): \hat{\lambda}^{T} x=\hat{\lambda}_{0}\right\} \subseteq$ $F$, where $\hat{\lambda}^{T} x \geq \hat{\lambda}_{0}$ corresponds to Inequality (9.1). To show the null coefficients of $\left(\lambda, \lambda_{0}\right)$, consider vertices $v \in V^{\prime}, u \in \bar{N}^{-}(v)$, and an integer $i \in[k]$. First, suppose that $v \notin H \backslash M_{H}$. Recall that $C^{u, i}$ denotes a $k$-fold coloring (with respect to $H$ ) that optimally colors $G[H]$, where $u$ does not share its $i$-th color with other vertices and every vertex not belonging to $H$ does not share any of its colors with other vertices. In particular, $S_{i}^{u}=\{u\}$ and $S_{i}^{w}=\{w\}$, for all $w \notin H$. So, one may easily check that the vectors $X\left(C^{u, i}\right)$ and $X\left(C^{u, i}\right)+e_{u v}^{i}$ belong to the face $\hat{F}$, which shows that $\lambda_{u v}^{i}=0$. If $v \notin H$, then $X\left(C^{u, i}\right)+e_{u v}^{i}-e_{v v}^{i}$ is also in $\hat{F}$. If $v \in H$, then $v \in M_{H}$, and so $u \notin H$. Therefore, $X\left(C^{v, i}\right)$ and $X\left(C^{v, i}\right)+e_{u v}^{i}-e_{v v}^{i}$ are in $\hat{F}$. In both cases, we conclude that $\lambda_{v v}^{i}=0$. Thus, we obtain $\lambda_{u v}^{i}=0$ for all $v \notin H \backslash M_{H}, u \in \bar{N}^{-}(v) \cup\{v\}$, and $i \in[k]$. Suppose now that $v \in H \backslash M_{H}$ and $u \in H$. Thus, we deduce that $X\left(C^{u, i}\right)$ and $X\left(C^{u, i}\right)+e_{u v}^{i}$ are in $\hat{F}$. Therefore, we have $\lambda_{u v}^{i}=0$ for all $v \in H \backslash M_{H}, u \in \bar{N}^{-}(v) \cap H$ and $i \in[k]$.

Consider now $v \in H \backslash M_{H}$ and $u \in \bar{N}^{-}(v) \backslash H$. For all $i, j \in[k]$, the vectors $X\left(C^{v, i}\right)$ and $X\left(C^{v, i}\right)-$ $e_{v v}^{i}+e_{u v}^{j}$ belong to $\hat{F}$. Thus, we have $\lambda_{u v}^{j}=\lambda_{v v}^{i}$ for all $v \in H \backslash M_{H}, u \in \bar{N}^{-}(v) \backslash H$ and $i, j \in[k]$. Henceforth, we refer to these coefficients as $\lambda_{v}$, that is, $\lambda_{v}=\lambda_{v v}^{i}$ for all $v \in H \backslash M_{H}$ and $i \in[k]$.

Remark 9.6. The results obtained until this point have only assumed that $G[H]$ is strongly $\chi_{k}$-critical. They hold for both strongly $\chi_{k}$-critical webs and antiwebs.

It remains to prove that $\lambda_{u}=\lambda_{v}$, for all $u, v \in H \backslash M_{H}$. Let $W_{p}^{n}$ be the strongly $\chi_{k}$-critical web in $G$ induced by the set $H$. Before showing the equality between the coefficients of vertices in $H \backslash M_{H}$, we prove the following assertion.

Claim 9.7. Let $w \in H \backslash M_{H}$ and $z \in \bar{N}^{-}(w) \cap\left(H \backslash M_{H}\right)$. If there exists a critical $k$-fold coloring $C^{w, 1}$ such that $z$ is not the representative of some of the color classes containing it, then $\lambda_{w}=\lambda_{z}$. Otherwise, there is a critical $k$-fold coloring $C^{w, 1}=\left\langle S_{1}, S_{2}, \ldots, S_{t}\right\rangle$ such that $S_{1}=\{w\}, z \in S_{2}$ and

(a) $\lambda_{w}=\lambda_{u}$ for all $u \in\left(S_{2} \backslash\{z\}\right) \cap \bar{N}^{-}(w)$, and

(b) $\lambda_{z}=\lambda_{u}$ for some $u \in \bar{N}(w) \cap \bar{N}^{+}(z) \cap S_{2}$.

Proof. If there exists a critical $k$-fold coloring $C^{w, 1}$ of $G$ with respect to $G[H]$ such that $z$ is not the representative of $S_{q}^{z}\left[C^{w, 1}\right]$, for some $q \in[k]$, then we have $X\left(C^{w, 1}\right), X\left(C^{w, 1}\right)-e_{w w}^{1}+e_{z z}^{q}+e_{z w}^{q} \in \hat{F}$ which imply $\lambda_{w}=\lambda_{z}$. Observe that $X\left(C^{w, 1}\right)-e_{w w}^{1}+e_{z z}^{q}+e_{z w}^{q}$ describes the $k$-fold coloring obtained from $C^{w, 1}$ by removing one of the color classes $\{w\}$ and adding the color class $\{z, w\}$ which is be represented by $z$.

Suppose now that, in any critical $k$-fold coloring $C^{w, 1}$ of $G$, vertex $z$ is the representative of all the color classes containing $z$. Without loss of generality, we can label the vertices of $G[H]=W_{p}^{n}$ in such a way that $v_{i}=w, v_{j}=z$ and $v_{j}$ is on the right of $v_{i}$, for some $i, j \in\{0,1, \ldots, n-1\}, i \neq j$. Thus, there is a critical $k$-fold coloring $C^{w, 1}$ such that $S_{1}\left[C^{w, 1}\right]=\{w\}, z \in S_{2}\left[C^{w, 1}\right]=\left\langle v_{i \oplus 1}, \ldots, v_{j}, \ldots, v_{i \oplus p}\right\rangle$, and $v_{j}$ is the smallest vertex in this color class.

Note that, for each $u \in\left(S_{2}\left[C^{w, 1}\right] \backslash\{z\}\right) \cap \bar{N}^{-}(w)$, we have $X\left(C^{w, 1}\right), X\left(C^{w, 1}\right)-e_{w w}^{1}+e_{u u}^{1}+e_{u w}^{1} \in \hat{F}$, and thus $\lambda_{w w}^{1}=\lambda_{u u}^{1}$. Therefore, we conclude that $\lambda_{w}=\lambda_{u}$, for all $u \in\left(S_{2}\left[C^{w, 1}\right] \backslash\{z\}\right) \cap \bar{N}^{-}(w)$. This shows item (a).

To show item (b), let $v_{\ell}$ be a vertex in $\bar{N}^{-}\left(v_{j}\right) \cap H$ such that the value $\min \{n-|j-\ell|,|j-\ell|\}$ is minimized. Note that $v_{\ell} \notin S_{2}\left[C^{v_{i}, 1}\right]$ since $v_{\ell} \prec v_{j}$. Let us consider the following two cases. 


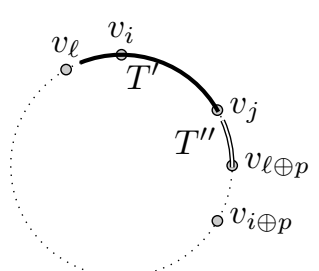

(a) $v_{\ell}$ is on the left of $v_{j}$.

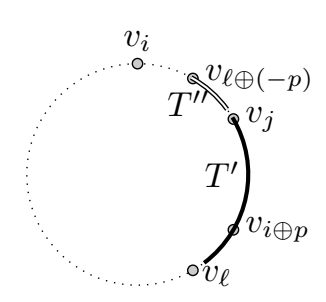

(b) $v_{\ell}$ is on the right of $v_{j}$.

Figure 9.2: The sets $T^{\prime}$ and $T^{\prime \prime}$

If $v_{\ell}$ is on the left of $v_{j}$, then it is also on the left of $v_{i}$. See Figure 9.2(a). Consider a critical $k$-fold coloring $C^{v_{\ell}, 1}$ such that $S_{1}\left[C^{v_{\ell}, 1}\right]=\left\{v_{\ell}\right\}$ and $S_{2}\left[C^{v_{\ell}, 1}\right]=T^{\prime} \cup T^{\prime \prime}$, where $T^{\prime}=\left\{v_{\ell \oplus 1}, \ldots, v_{i}, \ldots, v_{j}\right\}$ and $T^{\prime \prime}=\left\{v_{j \oplus 1}, \ldots, v_{\ell \oplus p}\right\}$. Due to the choice of $v_{\ell}$, every vertex on the right of $v_{\ell}$ and on the left of $v_{j}$ is greater than $v_{j}$. Thus, we have $T^{\prime} \backslash\left\{v_{j}\right\} \subseteq \bar{N}^{+}\left(v_{j}\right)$. In addition, $T^{\prime \prime}$ is non-empty and $T^{\prime \prime} \subseteq S_{2}\left[C^{v_{i}, 1}\right]$ since $v_{j} v_{\ell} \notin E(G)$ and $v_{i} \in S_{2}\left[C^{v_{\ell}, 1}\right]$. Therefore, $v_{j}$ is the representative of $S_{2}\left[C^{v_{\ell}, 1}\right]$.

If $v_{\ell}$ is on the right of $v_{j}$, it is also on the right of $v_{i \oplus p}$. See Figure 9.2(b). Consider a critical $k$-fold coloring $C^{v_{\ell}, 1}$ such that $S_{1}\left[C^{v_{\ell}, 1}\right]=\left\{v_{\ell}\right\}$ and $S_{2}\left[C^{v_{\ell}, 1}\right]=T^{\prime} \cup T^{\prime \prime}$, where $T^{\prime}=\left\{v_{\ell \oplus(-1)}, \ldots, v_{j}\right\}$ and $T^{\prime \prime}=\left\{v_{j \oplus(-1)}, \ldots, v_{\ell \oplus(-p)}\right\}$. Again due the choice of $v_{\ell}$, every vertex on its left and on the right of $v_{j}$ is greater than $v_{j}$. Thus, we have $T^{\prime} \backslash\left\{v_{j}\right\} \subseteq \bar{N}^{+}\left(v_{j}\right)$. In addition, $T^{\prime \prime}$ is a non-empty set and $T^{\prime \prime} \subseteq S_{2}\left[C^{v_{i}, 1}\right]$, since $v_{j} v_{\ell} \notin E(G)$ and every vertex in $T^{\prime \prime}$ is on the right of $v_{i}$ and on the left of $v_{j}$. Therefore, $v_{j}$ is the representative of $S_{2}\left[C^{v_{\ell}, 1}\right]$.

In either case, let $u$ be the smallest vertex in $T^{\prime \prime}$. Observe that $u \in \bar{N}\left(v_{i}\right) \cap S_{2}\left[C^{v_{i}, 1}\right]$ (i.e., $u \in$ $\left.\bar{N}(w) \cap S_{2}\left[C^{w, 1}\right]\right)$ and that $u$ is not the representative of its first color class in the coloring $C^{v_{\ell}, 1}$. Thus, we have that $X\left(C^{v_{\ell}, 1}\right)$ and

$$
X\left(C^{v_{\ell}, 1}\right)-e_{v_{j} v_{j}}^{1}-\sum_{y \in\left(T^{\prime} \cup T^{\prime \prime}\right) \backslash\left\{v_{j}\right\}} e_{v_{j} y}^{1}+\sum_{y \in T^{\prime}} e_{v_{\ell} y}^{1}+e_{u u}^{1}+\sum_{y \in T^{\prime \prime} \backslash\{u\}} e_{u y}^{1}
$$

belong to $\hat{F}$. The second vector describes the coloring obtained from $C^{v_{\ell}, 1}$ by transforming the color classes $\left\{v_{\ell}\right\}$ and $T^{\prime} \cup T^{\prime \prime}$ (represented by $v_{j}$ ) into the color classes $\left\{v_{\ell}\right\} \cup T^{\prime}$ and $T^{\prime \prime}$ (to be represented by $v_{\ell}$ and $u$, respectively). We have already proved that $\lambda_{u v}^{1}=0$ for all $v \in H \backslash M_{H}, u \in \bar{N}^{-}(v) \cap H$. So, in particular, $\lambda_{v_{j} y}^{1}=0$ for all $y \in\left(T^{\prime} \cup T^{\prime \prime}\right) \backslash\left\{v_{j}\right\}, \lambda_{v_{\ell} y}^{1}=0$ for all $y \in T^{\prime}$, and $\lambda_{u y}^{1}=0$ for all $y \in T^{\prime \prime} \backslash\{u\}$. Therefore, $\lambda_{v_{j} v_{j}}^{1}=\lambda_{u u}^{1}$, which implies $\lambda_{z}=\lambda_{u}$.

By induction on the order $\prec$, we shall prove that $\lambda_{z}=\lambda_{w}$, for all $w \in H \backslash M_{H}$ and $z \in \bar{N}^{-}(w) \cap$ $\left(H \backslash M_{H}\right)$. We fix vertices $w$ and $z$ in these sets. For the inductive basis, assume that $w$ is the greatest vertex in $H \backslash M_{H}$. If there exists a $k$-fold coloring $C^{w, 1}$ such that $z$ is not the representative of some of the color classes to which it belongs, then $\lambda_{z}=\lambda_{w}$ by Claim 9.7, and so we are done. Otherwise, by Claim 9.7(b), there is a coloring $C^{w, 1}=\left\langle S_{1}, \ldots, S_{t}\right\rangle$, where $S_{1}=\{w\}, z \in S_{2}$ and $\lambda_{z}=\lambda_{u}$, for some $u \in \bar{N}(w) \cap \bar{N}^{+}(z) \cap S_{2}$. Since $w$ is the greatest vertex in $H \backslash M_{H}, u \prec w$ and so $u \in\left(S_{2} \backslash\{z\}\right) \cap \bar{N}^{-}(w)$. By Claim 9.7(a), we get $\lambda_{w}=\lambda_{u}$, and thus $\lambda_{z}=\lambda_{w}$. Suppose now that $w$ is not the greatest vertex in $H \backslash M_{H}$, and that $\lambda_{x}=\lambda_{y}$, for all $x \in H \backslash M_{H}$ such that $x \succ w$ and for all $y \in \bar{N}^{-}(x) \cap\left(H \backslash M_{H}\right)$. Likewise in the inductive basis, we can use Claim 9.7 to show that $\lambda_{w}=\lambda_{z}$ except if the vertex $u$ given by Claim 9.7(b) is such that $u \succ w$. In this remaining case, $w, z \in \bar{N}^{-}(u) \cap\left(H \backslash M_{H}\right)$, since $u \in \bar{N}^{+}(w) \cap \bar{N}^{+}(z) \cap S_{2}$. By the inductive hypothesis, we obtain $\lambda_{w}=\lambda_{u}$ and $\lambda_{z}=\lambda_{u}$. Therefore, we have $\lambda_{z}=\lambda_{w}$, for all $w \in H \backslash M_{H}$ and $z \in \bar{N}^{-}(w) \cap\left(H \backslash M_{H}\right)$. 
Finally, we extend the equality $\lambda_{w}=\lambda_{z}$ to every $w, z \in H \backslash M_{H}$. Since $W_{p}^{n}$ is strongly $\chi_{k}$-critical, Theorem 9.1 implies $n>2 p$. Also, recall that $p \geq 2$. If $p>2$, then $v_{i \oplus(-1)} v_{i \oplus 1}$ and $v_{i} v_{i \oplus 1}$ are not in $E\left(W_{p}^{n}\right)$, for each $i \in\{0,1, \ldots, n-1\}$. Thus, $\bar{W}_{p}^{n} \backslash M_{H}$ is connected since $M_{H}$ induces a clique in $W_{p}^{n}$. Hence, $\lambda_{w}=\lambda_{z}$ directly extends to every $w, z \in H \backslash M_{H}$. If $p=2$, then $\bar{W}_{p}^{n}$ is a cycle, and so $\bar{W}_{p}^{n} \backslash M_{H}$ is not connected. However, if $V_{1}, V_{2}$ are the sets of vertices of two distinct components (paths) in $\bar{W}_{p}^{n} \backslash M_{H}$, then there exist $w \in V_{1}, z \in V_{2}$ and $y \in M_{H}$ such that $w, z \in \bar{N}^{+}(y)$. Note that there exists a critical $k$-fold coloring $C^{w, 1}$ such that $S_{1}=\{w\}$ and $S_{2}=\{y, z\}$. Therefore, $X\left(C^{w, 1}\right)$ and $X\left(C^{w, 1}\right)-e_{w w}^{1}+e_{y w}^{1}+e_{z z}^{1}-e_{y z}^{1}$ belong to $\hat{F}$, which yields $\lambda_{w}=\lambda_{z}$. Again, this equality extends to all $w, z \in H \backslash M_{H}$.

\subsection{Antiweb facets}

Before characterizing the strongly critical antiwebs, we present an important definition that is used in this section. For each $i \in \mathbb{N} \cup\{0\}$, we define

$$
\mathbf{S}_{i}= \begin{cases}\left\langle x(t): t \in\left\{0,1, \ldots, \alpha\left(\bar{W}_{p}^{n}\right)-1\right\}\right\rangle & \text { if } i=0 \\ \left\langle j \oplus i: j \in \mathrm{S}_{0}\right\rangle & \text { otherwise. }\end{cases}
$$

where

$$
x(t)=\left\lceil\frac{t n}{\alpha\left(\bar{W}_{p}^{n}\right)}\right\rceil \text { for every } t \in \mathbb{N} \cup\{0\} .
$$

Campêlo et al. [18] showed that each $\mathrm{S}_{i}$ induces a maximum stable set of $\bar{W}_{p}^{n}$. Furthermore, they proved the following lemma.

Lemma 9.8 (Campêlo et al. [18]). The index sets $\mathrm{S}_{0}, \mathrm{~S}_{1}, \ldots, \mathrm{S}_{n-1}$ induce an $\alpha\left(\bar{W}_{p}^{n}\right)$-fold n-coloring of $\bar{W}_{p}^{n}$.

Lemma 9.9. For every $k \in \mathbb{N}$, if $\frac{k n-1}{\alpha\left(\bar{W}_{p}^{n}\right)} \in \mathbb{Z}$, then $\bar{W}_{p}^{n}$ is strongly $\chi_{k}$-critical.

Proof. Observe that, since $n \geq 2 p$ and $\alpha\left(\bar{W}_{p}^{n}\right)=\left\lfloor\frac{n}{p}\right\rfloor$, we have $2 \leq \alpha\left(\bar{W}_{p}^{n}\right) \leq n-1$. For each $i \in \mathbb{N} \cup\{0\}$ we define

$$
\bar{x}(i)=\left\lceil\frac{i n-1}{\alpha\left(\bar{W}_{p}^{n}\right)}\right\rceil .
$$

Firstly, consider that $k \leq \alpha\left(\bar{W}_{p}^{n}\right)$. Let $\ell \in[k]$, and $t \in\left[\alpha\left(\bar{W}_{p}^{n}\right)-1\right] \cup\{0\}$. Note that $\bar{x}(\ell) \geq 1$. We define $A(\ell, t)$ as the sequence comprising the $(t+1)$-th elements of $\mathrm{S}_{0}, \mathrm{~S}_{1}, \ldots, \mathrm{S}_{\bar{x}(\ell)-1}$, that is,

$$
A(\ell, t)=\langle x(t) \oplus i: i \in\{0,1, \ldots, \bar{x}(\ell)-1\}\rangle
$$

Since $\ell \leq \alpha\left(\bar{W}_{p}^{n}\right), A(\ell, t)$ contains $\bar{x}(\ell)$ distinct elements.

Claim 9.10. $1 \leq \bar{x}(t+\ell)-x(t) \leq \bar{x}(\ell)$

Proof. It is know that, if $x, y \in \mathbb{R}$ and $x \geq y$, then $\lfloor x-y\rfloor \leq\lceil x\rceil-\lceil y\rceil \leq\lceil x-y\rceil[18]$. Let $x=\bar{x}(t+\ell)$ and $y=x(t)$. Thus, we directly obtain $\bar{x}(t+\ell)-x(t) \leq \bar{x}(\ell)$. On the other hand, $\bar{x}(t+\ell)-x(t) \geq\left\lfloor\frac{\ell n-1}{\alpha\left(\bar{W}_{p}^{n}\right)}\right\rfloor \geq 1$, where the last inequality holds because $\ell \geq 1$ and $\alpha\left(\bar{W}_{p}^{n}\right) \leq n-1$.

By Claim 9.10, we can define $B(\ell, t)$ as the subsequence of $A(\ell, t)$ formed by its first $\bar{x}(t+\ell)-x(t)$ elements. Observe that $B(\ell, t)$ comprises consecutive integers (modulus $n$ ), starting at $x(t) \oplus 0$ and ending 
at $\bar{x}(t+\ell) \oplus(-1)$. Thus, we have $B(\ell, t) \subseteq B(\ell+1, t)$. Let $C(1, t)=B(1, t)$ and $C(\ell, t)=B(\ell, t) \backslash B(\ell-1, t)$, for $2 \leq \ell \leq k$.

First consider that $\ell \geq 2$. Similarly to $B(\ell, t), C(\ell, t)$ comprises consecutive integers (modulus $n$ ), starting at $\bar{x}(t+\ell-1) \oplus 0$ and ending at $\bar{x}(t+\ell) \oplus(-1)$. Observe that the first element of $C(\ell, t+1)$ is the last element of $C(\ell, t)$ plus 1 (modulus $n$ ). Thus, $C(\ell)=\left\langle C(\ell, 0), C(\ell, 1), \ldots, C\left(\ell, \alpha\left(\bar{W}_{p}^{n}\right)-1\right)\right\rangle$ is a sequence of consecutive integers (modulus $n$ ) starting at the first element of $C(\ell, 0)$, that is, $\bar{x}(\ell-1) \oplus 0$, and ending at the last element of $C\left(\ell, \alpha\left(\bar{W}_{p}^{n}\right)-1\right)$, that is, $\bar{x}\left(\alpha\left(\bar{W}_{p}^{n}\right)+\ell-1\right) \oplus(-1)=\bar{x}(\ell-1) \oplus(-1)$. Therefore, for each $\ell \in\{2, \ldots, k\}, C(\ell) \equiv\langle 0,1, \ldots, n-1\rangle$.

Now assume that $\ell=1$. Recall that $C(1, t)=B(1, t)$ comprises consecutive integers (modulus $n$ ), starting at $x(t) \oplus 0$ and ending at $\bar{x}(t+1) \oplus(-1)$. We shall prove that

$$
C(1)=\left\langle C(1,0), C(1,1), \ldots, C\left(1, \alpha\left(\bar{W}_{p}^{n}\right)-1\right)\right\rangle
$$

contains all but one element from $\langle 0,1, \ldots, n-1\rangle$.

Claim 9.11. If $t \neq k$, then $x(t)=\bar{x}(t)$.

Proof. Let $j \in \mathbb{Z}$ such that $0<|j|<\alpha\left(\bar{W}_{p}^{n}\right)$. Suppose to the contrary that $\frac{j n}{\alpha\left(\bar{W}_{p}^{n}\right)} \in \mathbb{Z}$. Since $\frac{k n-1}{\alpha\left(\bar{W}_{p}^{n}\right)} \in \mathbb{Z}$, we have $\frac{j k n-j}{\alpha\left(\bar{W}_{p}^{n}\right)}=k \cdot \frac{j n}{\alpha\left(\bar{W}_{p}^{n}\right)}-\frac{j}{\alpha\left(\bar{W}_{p}^{n}\right)} \in \mathbb{Z}$. This implies $\frac{j}{\alpha\left(\bar{W}_{p}^{n}\right)} \in \mathbb{Z}$, a contradiction. Consequently, we have $\frac{j n}{\alpha\left(\bar{W}_{p}^{n}\right)} \notin \mathbb{Z}$, and so

$$
\left\lceil\frac{j n}{\alpha\left(\bar{W}_{p}^{n}\right)}\right\rceil=\left\lceil\frac{j n+1}{\alpha\left(\bar{W}_{p}^{n}\right)}\right\rceil, \text { for every } j \in \mathbb{Z} \text { such that } 0<|j|<\alpha\left(\bar{W}_{p}^{n}\right) .
$$

Note that $0<|t-k|<\alpha\left(\bar{W}_{p}^{n}\right)$ because $t \neq k, 0 \leq t<\alpha\left(\bar{W}_{p}^{n}\right)$ and $1 \leq k<\alpha\left(\bar{W}_{p}^{n}\right)\left(k \neq \alpha\left(\bar{W}_{p}^{n}\right)\right.$ is due to $\left.\frac{k n-1}{\alpha\left(\overline{W_{p}^{n}}\right)} \in \mathbb{Z}\right)$. Therefore,

$$
\left\lceil\frac{(t-k) n}{\alpha\left(\bar{W}_{p}^{n}\right)}\right\rceil=\left\lceil\frac{(t-k) n+1}{\alpha\left(\bar{W}_{p}^{n}\right)}\right\rceil
$$

or still

$$
\left\lceil\frac{t n-1}{\alpha\left(\bar{W}_{p}^{n}\right)}\right\rceil=\left\lceil\frac{t n}{\alpha\left(\bar{W}_{p}^{n}\right)}\right\rceil
$$

which shows that $\bar{x}(t)=x(t)$.

By Claim 9.11, the first element of $C(1, t+1)$ is the last element of $C(1, t)$ plus 1 (modulus $n$ ) if $0 \leq$ $t \leq k-2$ or $k \leq t \leq \alpha\left(\bar{W}_{p}^{n}\right)-2$. This implies that $\langle C(1,0), C(1,1), \ldots, C(1, k-1)\rangle$ and $\langle C(1, k), C(1, k+$ $\left.1), \ldots, C\left(1, \alpha\left(\bar{W}_{p}^{n}\right)-1\right)\right\rangle$ are two sequences of consecutive integers. Moreover, the last element of $C(1, k-1)$ is $\bar{x}(k) \oplus(-1)=\frac{k n-1}{\alpha\left(\overline{\left.W_{p}^{n}\right)}\right.} \oplus(-1)$, and the first element of $C(1, k)$ is $x(k) \oplus 0=\left\lceil\frac{k n}{\alpha\left(\bar{W}_{p}^{n}\right)}\right\rceil \oplus 0=\frac{k n-1}{\alpha\left(\bar{W}_{p}^{n}\right)} \oplus 1=$ $\bar{x}(k) \oplus 1$. It follows that

$$
C(1) \equiv\langle 0,1, \ldots, \bar{x}(k) \oplus(-1), \bar{x}(k) \oplus 1, \ldots n-1\rangle .
$$

Therefore, $C(1)$ contains every vertex index except $\bar{x}(k) \oplus 0$ exactly once. Moreover, for each $\ell \in$ $\{2, \ldots, k\}, C(\ell)$ contains every vertex index once. In addition, by definition of $C(\ell, t)$, for $\ell>1$, the sequences $\mathrm{S}_{i}$ 's from which the elements of $C(\ell, t)$ are taken are different from those of $C(1, t), C(2, t), \ldots, C(\ell-$ $1, t)$. Consequently, every vertex index appears $k-1$ times in the sequences $C(2), \ldots, C(k)$, and every 
vertex index except $\bar{x}(k) \oplus 0$ appears one more time in $C(1)$. It follows that the sets $\mathrm{S}_{0}, \mathrm{~S}_{1}, \ldots, \mathrm{S}_{\bar{x}(k)-1}$ and $\{\bar{x}(k) \oplus 0\}$ induce a $k$-fold coloring of $\bar{W}_{p}^{n}$ with $\bar{x}(k)+1=x(k)$ colors, which is therefore optimal [18]. This completes the case $k \leq \alpha\left(\bar{W}_{p}^{n}\right)$.

If $k>\alpha\left(\bar{W}_{p}^{n}\right)$, then we write $k=q \alpha\left(\bar{W}_{p}^{n}\right)+r$, for integers $q \geq 1$ and $0 \leq r<\alpha\left(\bar{W}_{p}^{n}\right)$. Since $\frac{k n-1}{\alpha\left(\bar{W}_{p}^{n}\right)} \in \mathbb{Z}$, we have $\frac{r n-1}{\alpha\left(\bar{W}_{p}^{n}\right)} \in \mathbb{Z}$. Thus, we have proved that the sets $\mathrm{S}_{0}, \mathrm{~S}_{1}, \ldots, \mathrm{S}_{\bar{x}(r)-1},\{\bar{x}(r) \oplus 0\}$ induce an $r$ fold $(\bar{x}(r)+1)$-coloring of $\bar{W}_{p}^{n}$. By Lemma 9.8 , the index sets $\mathrm{S}_{0}, \ldots, \mathrm{S}_{n-1}$ induce an $\alpha\left(\bar{W}_{p}^{n}\right)$-fold $n$ coloring. One copy of the first coloring together with $q$ copies of the second one yield a $k$-fold coloring with $q n+\frac{r n-1}{\alpha\left(\bar{W}_{p}^{n}\right)}+1=\frac{k n-1}{\alpha\left(\bar{W}_{p}^{n}\right)}+1=\left\lceil\frac{k n}{\alpha\left(\bar{W}_{p}^{n}\right)}\right\rceil$ colors. In other words, there exists an optimal $k$-fold coloring of $\bar{W}_{p}^{n}$ in which the singleton set $\left\{v_{z}\right\}$ is a color class, where $z=\bar{x}(r) \oplus 0$.

Due to the symmetry of antiwebs, we conclude that $\bar{W}_{p}^{n}$ is strongly $\chi_{k}$-critical.

Remark 9.12. Let $k=q \alpha\left(\bar{W}_{p}^{n}\right)+r$, with $q \geq 0$ and $0 \leq r<\alpha\left(\bar{W}_{p}^{n}\right)$. If $i=\bar{x}(r) \oplus j$, for some $j \in \mathbb{Z}$, an optimal $k$-fold coloring where $\left\{v_{i}\right\}$ is a color class is induced by $q$ copies of $\mathrm{S}_{0}, \ldots, \mathrm{S}_{n-1}$ together with $\mathrm{S}_{0 \oplus j}, \mathrm{~S}_{1 \oplus j}, \ldots, \mathrm{S}_{(\bar{x}(r)-1) \oplus j},\{\bar{x}(r) \oplus j\}$.

As in the case of webs, we show facetness of Inequality (9.1) for an antiweb $G[H]$ by means of critical $k$-fold colorings with respect to $H$. These colorings may be derived from those described in the previous remark.

Lemma 9.13. Let $k \in \mathbb{N}, H \subseteq V(G)$ and $M_{H}$ be the set of sources in the suborder of $\prec$ restricted to $H$. If $G[H]$ is a strongly $\chi_{k}$-critical antiweb, then Inequality (9.1) induces a facet of $\mathcal{P}_{k}(G)$.

Proof. Let $\bar{W}_{p}^{n}$ be the antiweb graph induced by $H$, that is, $\bar{W}_{p}^{n}=G[H]$. Since $n \geq 2 p$ and $\alpha\left(\bar{W}_{p}^{n}\right)=\left\lfloor\frac{n}{p}\right\rfloor$, we have $\alpha\left(\bar{W}_{p}^{n}\right) \geq 2$. If $\alpha\left(\bar{W}_{p}^{n}\right)=2$, then $W_{p}^{n}$ is a hole. Moreover, since $\frac{n k-1}{2} \in \mathbb{Z}, n$ must be odd. Therefore, $\bar{W}_{p}^{n}$ is an odd anti-hole, and so it is also a web graph. By Lemma 9.5, the result follows. So, from now on assume that $\alpha\left(\bar{W}_{p}^{n}\right) \geq 3$.

Consider a face $F=\left\{x \in P_{k}(G): \lambda^{T} x=\lambda_{0}\right\}$ of $P_{k}(G)$ such that $\hat{F}=\left\{x \in P_{k}(G): \hat{\lambda}^{T} x=\hat{\lambda}_{0}\right\} \subseteq F$, where $\hat{\lambda}^{T} x \geq \hat{\lambda}_{0}$ corresponds to Inequality (9.1).

As anticipated in Remark 9.6, one may show the null coefficients of $\left(\lambda, \lambda_{0}\right)$ in the same manner as in the proof of Lemma 9.5. Thus, we have $\lambda_{u v}^{i}=0$ for all $v \notin H \backslash M_{H}, u \in \bar{N}^{-}(v) \cup\{v\}$ and $i \in[k]$. Moreover, $\lambda_{u v}^{i}=0$ for all $v \in H \backslash M_{H}, u \in \bar{N}^{-}(v) \cap H$ and $i \in[k]$. Analogously, we obtain $\lambda_{u v}^{j}=\lambda_{v v}^{i}=: \lambda_{v}$ for all $v \in H \backslash M_{H}, u \in \bar{N}^{-}(v) \backslash H$ and $i, j \in[k]$. So, it remains to prove that $\lambda_{u}=\lambda_{v}$, for all $u, v \in H \backslash M_{H}$.

Given a pair of vertices $u$ and $v$ in $H \backslash M_{H}$, we shall prove the desired equality in three steps. First, we suppose that $u$ and $v$ are non-adjacent in $\bar{W}_{p}^{n}=G[H]$ and $\bar{N}^{-}(v) \cap \bar{N}^{-}(u) \cap H \neq \emptyset$. Using this result, we are then able to suppose the non-adjacency condition only. Finally, the condition is proved to be unnecessary by showing that $W_{p}^{n} \backslash M_{H}$ is connected. We repeatedly use the fact that $X(C) \in \hat{F}$ for any critical $k$-fold coloring $C$ of $G$ with respect to the subgraph $H$.

Claim 9.14. Let $u, v \in H \backslash M_{H}$. If $u v \notin E\left(\bar{W}_{p}^{n}\right)$ and $\bar{N}^{-}(v) \cap \bar{N}^{-}(u) \cap H \neq \emptyset$, then $\lambda_{u}=\lambda_{v}$.

Proof. Let $w \in \bar{N}^{-}(v) \cap \bar{N}^{-}(u) \cap H$. We assume, without loss of generality, that $u \prec v$. If $u$ is not the representative of some color class $S_{i}^{u}\left[C^{v, j}\right]$, where $i, j \in[k]$ and $C^{v, j}$ is a critical $k$-fold coloring, then the vectors $X\left(C^{v, j}\right)$ and $X\left(C^{v, j}\right)-e_{v v}^{j}+e_{u u}^{i}+e_{u v}^{i}$ belong to $\hat{F}$. Thus, we have $\lambda_{u}=\lambda_{v}$ since $\lambda_{u v}^{i}=0$.

In the rest of this proof, assume that $u$ is the representative of each color class in $S^{u}\left[C^{v, j}\right]$, where $j \in[k]$ and $C^{v, j}$ is a critical $k$-fold coloring. Let $z \in H$ be the smallest vertex in the ordering $\prec$ that belongs to $S_{1}^{u} \backslash\{u\}$. There exists such a vertex $z$ because, as observed in the proof of Theorem 9.1, every color 
class in $C^{v, j}$ has size $\alpha(G[H]) \geq 2$ except for $S_{j}^{v}$, which contains only the vertex $v$. Moreover, $z$ is not the representative of the color class $S_{1}^{u}=S_{i}^{z}$, for some $i \in[k]$. One may check that $X\left(C^{v, j}\right)$ and

$$
X\left(C^{v, j}\right)-e_{v v}^{j}-\sum_{y \in S_{1}^{u} \backslash\{u\}} e_{u y}^{1}+e_{u v}^{1}+e_{z z}^{i}+\sum_{y \in S_{1}^{u} \backslash\{u, z\}} e_{z y}^{i}
$$

belong to $\hat{F}$. The second vector describes the coloring obtained from $C^{v, j}$ by moving $u$ from color class $S_{i}^{z}$ to color class $\{v\}$. Consequently, we have $\lambda_{z}=\lambda_{v}$. Consider now the following two cases.

If $w$ is not the representative of the color class $S_{\ell}^{w}$ in the critical $k$-fold coloring $C^{v, j}$, for some $\ell \in$ $[k]$, then the vectors $X\left(C^{v, j}\right)$ and $X\left(C^{v, j}\right)-e_{v v}^{j}+e_{w w}^{\ell}+e_{w v}^{\ell}$ are in $\hat{F}$, so $\lambda_{w}=\lambda_{v}$. Moreover, the vectors $X\left(C^{v, j}\right)$ and

$$
X\left(C^{v, j}\right)-e_{v v}^{j}-e_{u u}^{1}-\sum_{y \in S_{1}^{u} \backslash\{u\}} e_{u y}^{1}+e_{z z}^{i}+\sum_{y \in S_{1}^{u} \backslash\{u, z\}} e_{z y}^{i}+e_{w w}^{\ell}+e_{w u}^{\ell}+e_{w v}^{\ell}
$$

belong to the face $\hat{F}$. Thus, these vectors show that $\lambda_{v}+\lambda_{u}=\lambda_{z}+\lambda_{w}$. Therefore, we deduce that $\lambda_{u}=\lambda_{v}$.

If $w$ is the representative of each color class in $S^{w}\left[C^{v, j}\right]$, then let $z^{\prime}$ be the smallest vertex in $S_{1}^{w} \backslash\{w\}$. Note that $z^{\prime}$ is not the representative of the color class $S_{1}^{w}=S_{p}^{z^{\prime}}$, for some $p \in[k]$. One may verify that $X\left(C^{v, j}\right)$ and

$$
X\left(C^{v, j}\right)-e_{v v}^{j}-\sum_{y \in S_{1}^{w} \backslash\{w\}} e_{w y}^{1}+e_{w v}^{1}+e_{z^{\prime} z^{\prime}}^{p}+\sum_{y \in S_{1}^{w} \backslash\left\{w, z^{\prime}\right\}} e_{z^{\prime} y}^{p}
$$

are in $\hat{F}$, so $\lambda_{z^{\prime}}=\lambda_{v}$. Moreover, the vectors $X\left(C^{v, j}\right)$ and

$$
\begin{aligned}
X\left(C^{v, j}\right) & -e_{v v}^{j} \\
& -e_{u u}^{1}-\sum_{y \in S_{1}^{u} \backslash\{u\}} e_{u y}^{1} \\
& +e_{z z}^{i}+\sum_{y \in S_{1}^{u} \backslash\{u, z\}} e_{z y}^{i} \\
& +e_{z^{\prime} z^{\prime}}^{p}+\sum_{y \in S_{1}^{w} \backslash\left\{w, z^{\prime}\right\}} e_{z^{\prime} y}^{p} \\
& -\sum_{y \in S_{1}^{w} \backslash\{w\}} e_{w y}^{1} \\
& +e_{w v}^{1}+e_{w u}^{1}
\end{aligned}
$$

also belong to the face $\hat{F}$, and thus we have $\lambda_{v}+\lambda_{u}=\lambda_{z}+\lambda_{z^{\prime}}$. Therefore, we conclude that $\lambda_{u}=\lambda_{v}$.

Let $v \in H \backslash M_{H}$ and let $u \in \bar{N}^{-}(v) \cap\left(H \backslash M_{H}\right)$. We next prove that $\lambda_{u}=\lambda_{v}$. If $u$ is not the representative of some color class $S_{i}^{u}\left[C^{v, j}\right]$, where $i, j \in[k]$ and $C^{v, j}$ is a critical $k$-fold coloring, then the vectors $X\left(C^{v, j}\right)$ and $X\left(C^{v, j}\right)-e_{v v}^{j}+e_{u u}^{i}+e_{u v}^{i}$ show that $\lambda_{u}=\lambda_{v}$. Suppose now that $u$ is the representative of each color class in $S^{u}\left[C^{v, j}\right]$ for all $j \in[k]$, where $C^{v, j}$ is a critical $k$-fold coloring. We are able to prove the following claim.

Claim 9.15. For every $i, j \in[k]$, we have $\lambda_{v}=\lambda_{y}$ for all $y \in S_{i}^{u}\left[C^{v, j}\right] \backslash\{u\}$.

Proof. Let $S$ denote the color class $S_{i}^{u}\left[C^{v, j}\right]$. By Claim 9.14, it follows that $\lambda_{y}=\lambda_{y^{\prime}}$ for all $y, y^{\prime} \in S \backslash\{u\}$. Let $z$ be the smallest vertex in the ordering $\prec$ that belongs to $S \backslash\{u\}$. Since $u$ is the representative of $S$, 
there exists $\ell \in[k]$ such that $z$ is not the representative of $S_{\ell}^{z}$, where $S_{\ell}^{z}=S$. One may easily verify that the vectors $X\left(C^{v, j}\right)$ and

$$
X\left(C^{v, j}\right)-e_{v v}^{j}+e_{u v}^{i}-\sum_{y \in S \backslash\{u\}} e_{u y}^{i}+e_{z z}^{\ell}+\sum_{y \in S \backslash\{u, z\}} e_{z y}^{\ell}
$$

belong to $\hat{F}$. Thus, we have $\lambda_{v}=\lambda_{z}$, and so $\lambda_{v}=\lambda_{y}$ for all $y \in S \backslash\{u\}$.

We consider the following two subcases.

If $\alpha(G[H]) \geq 4$, then let $w$ be a vertex in $\bar{N}^{-}(u) \cap H$. One may easily verify that every vertex in an antiweb graph has at most two neighbors in the same stable set. Thus, since $\alpha(G[H]) \geq 4$, there exist $z \in S_{1}^{u} \backslash u$ such that $z$ is not adjacent to $w$. Since $u$ is the representative of $S_{1}^{u}$, we have $z \succ u$, and thus $w \prec u \prec z$. By Claim 9.14, we have $\lambda_{u}=\lambda_{z}$. Therefore, we conclude that $\lambda_{u}=\lambda_{v}$ by Claim 9.15.

Suppose now that $\alpha(G[H])=3$. Let $C^{v, 1}$ be the critical $k$-fold coloring of $G[H]$ with color class $\{v\}$ as given by Remark 9.12. Let $i \in[n]$ be the index of $v$ in the antiweb $G[H]$, that is, $v=v_{i}$ where $v_{i}$ is a vertex of the antiweb induced by $H$. To complete the proof for this subcase, we need the following claim.

Claim 9.16. Let $v_{j} \in H$ be a neighbor of $v=v_{i}$ in $G[H]$. There exists a color class $S_{*}^{j}$ in $C^{v, 1}$ such that $v_{j} \in S_{*}^{j}$, and $\left(S_{*}^{j} \backslash\left\{v_{j}\right\}\right) \cup\left\{v_{\ell}\right\}$ is a stable set of $G[H]$, where $\ell=j \oplus 1$ if $v_{j}$ is on the left of $v$, and $\ell=j \oplus(-1)$ otherwise.

Proof. Due to the symmetry of $\bar{W}_{p}^{n}$, we can assume, with no loss of generality, that $i=\left\lceil\frac{n}{3}\right\rceil-1$. By Remark 9.12, $C^{v, 1}$ contains the color classes induced by the sets of indices $\mathrm{S}_{0}, \ldots, \mathrm{S}_{\left\lceil\frac{n}{3}\right\rceil-2},\{i\}$. We remark that $\left\lceil\frac{n}{3}\right\rceil \geq p+1$ since $\left\lfloor\frac{n}{p}\right\rfloor=3$ and $\frac{k n-1}{3} \in \mathbb{Z}$. Consider the following two cases.

If $v_{j}$ is on the left of $v$, then $j \in\{i-1, i-2, \ldots, i-(p-1)\} \subseteq\left\{1, \ldots,\left\lceil\frac{n}{3}\right\rceil-2\right\}$, because $v_{j} v \in E\left(\bar{W}_{p}^{n}\right)$. We have that $\mathrm{S}_{j}=\left\{j, j+\left\lceil\frac{n}{3}\right\rceil, j+\left\lceil\frac{2 n}{3}\right\rceil\right\}$. Choose $S_{*}^{j}$ as the stable set induced by $\mathrm{S}_{j}$. Thus, we obtain that

$$
\mathrm{S}_{j}^{\prime}=\left(\mathrm{S}_{j} \backslash\{j\}\right) \cup\{j+1\}=\left\{j+1, j+\left\lceil\frac{n}{3}\right\rceil, j+\left\lceil\frac{2 n}{3}\right\rceil\right\}
$$

also indexes a stable set of $\bar{W}_{p}^{n}$. Indeed, regarding $\mathrm{S}_{j}$, the maximum distance between two indices in $\mathrm{S}_{j}^{\prime}$ has decreased, whereas the minimum distance remains at least $p$, since $\left\lceil\frac{n}{3}\right\rceil-1 \geq p$.

Suppose now that $v_{j}$ is on the right of $v$. Thus, there is $q \in\{0, \ldots, p-2\}$ such that $j=i+1+q$. Let $S_{*}^{j}$ be the stable set induced by $\mathrm{S}_{q}=\left\{q, q+\left\lceil\frac{n}{3}\right\rceil, q+\left\lceil\frac{2 n}{3}\right\rceil\right\}$. Again,

$$
\mathrm{S}_{q}^{\prime}=\left(\mathrm{S}_{q} \backslash\{j\}\right) \cup\{j-1\}=\left\{q, q+\left\lceil\frac{n}{3}\right\rceil-1, q+\left\lceil\frac{2 n}{3}\right\rceil\right\}
$$

indexes a stable set of $\bar{W}_{p}^{n}$. The maximum distance between two indices in $\mathrm{S}_{q}^{\prime}$ is the same as in $\mathrm{S}_{q}$, whereas the minimum distance remains at least $p$, since $\left\lceil\frac{n}{3}\right\rceil-1 \geq p$.

If $\bar{N}^{-}(v) \cap \bar{N}^{-}(u) \cap H \neq \emptyset$, then we have $\lambda_{u}=\lambda_{v}$ by Claim 9.14. Thus, we now suppose that every vertex in $\bar{N}^{-}(u) \cap H$ is adjacent to $v$. Consider a vertex $v_{j} \in \bar{N}^{-}(u) \cap H$ such that the value $t:=$ $\min \{n-|i-j|,|i-j|\}$ is minimized. Let $W=\left\{w_{0}, w_{1}, \ldots, w_{t}\right\} \subset H$ be set of vertices between $v$ and $v_{j}$ in $G[H]$, where $v=w_{0}, v_{j}=w_{t}$, and $w_{\ell}=v_{i \oplus \ell}$ for all $\ell \in[t]$ if $v_{j}$ is on the right of $v$, and $w_{\ell}=v_{i \oplus(-\ell)}$ for all $\ell \in[t]$ otherwise. By Claim 9.16, for each $\ell \in[t]$, there exists a color class $\hat{S}_{*}^{\ell}$ in the coloring $C^{v, 1}$ such that $w_{\ell} \in \hat{S}_{*}^{\ell}$ and $\left(\hat{S}_{*}^{\ell} \backslash\left\{w_{\ell}\right\}\right) \cup\left\{w_{\ell-1}\right\}$ is a stable set of $G[H]$. Let $C^{\prime}$ be the family of stable sets of $G[H]$ that is obtained from $C^{v, 1}$ by replacing the color class $\hat{S}_{*}^{\ell}$ by $\left(\hat{S}_{*}^{\ell} \backslash\left\{w_{\ell}\right\}\right) \cup\left\{w_{\ell-1}\right\}$, for each $\ell \in[t]$, and replacing $\{v\}$ by $\left\{v_{j}\right\}$. Note that $\hat{S}_{*}^{\ell} \cap W=\left\{w_{\ell}\right\}$, for each $\ell \in[t]$, since $W$ induces a clique in $G[H]$. 


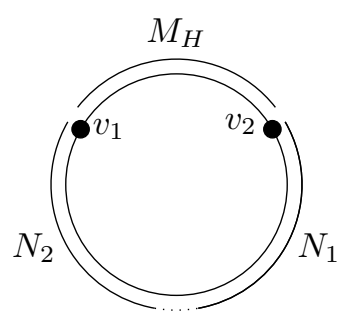

Figure 9.3: Illustration of $v_{1}, v_{2}, N_{1}$ and $N_{2}$.

Also, one may easily check that $C^{\prime}$ is a critical $k$-fold coloring of $G[H]$ with color class $\left\{v_{j}\right\}$. Due to the choice of $v_{j}$, it follows that $u$ is still the representative of every color class in $S^{u}\left[C^{\prime}\right]$.

Let $z \in H$ be the smallest vertex in the ordering $\prec$ that belongs to $S_{1}^{u}\left[C^{\prime}\right] \backslash\{u\}$, and let $q \in[k]$ be the index of the color class $S_{1}^{u}\left[C^{\prime}\right]$ of $z$, that is, $S_{q}^{z}\left[C^{\prime}\right]=S_{1}^{u}\left[C^{\prime}\right]$. By Claim 9.14, $\lambda_{z}=\lambda_{y}$ for all $y \in S_{1}^{u}\left[C^{\prime}\right] \backslash\{u\}$. Additionally, let $\ell \in[k]$ denote the index of the color class $\left\{v_{j}\right\}$ of $v_{j}$ in the coloring $C^{\prime}$. Note that the vectors $X\left(C^{\prime}\right)$ and

$$
X\left(C^{\prime}\right)+e_{v_{j} u}^{\ell}-e_{u u}^{1}-\sum_{y \in S_{1}^{u} \backslash\{u\}} e_{u y}^{1}+e_{z z}^{q}+\sum_{y \in S_{1}^{u} \backslash\{u, z\}} e_{z y}^{q}
$$

belong to $\hat{F}$. Thus, it follows that $\lambda_{u}=\lambda_{z}$, and so $\lambda_{u}=\lambda_{y}$ for all $y \in S_{1}^{u}\left[C^{\prime}\right]$. Since $\alpha(G[H])=3$, we obtain that $\left(S_{1}^{u}\left[C^{\prime}\right] \backslash\{u\}\right) \cap\left(S_{1}^{u}\left[C^{v, 1}\right] \backslash\{u\}\right) \neq \emptyset$. Therefore, we conclude that $\lambda_{u}=\lambda_{v}$ by Claim 9.15.

We have proved that $\lambda_{u}=\lambda_{v}$, for all $u, v \in H \backslash M_{H}$ such that $u$ and $v$ are non-adjacent in $G[H]=\bar{W}_{p}^{n}$. In order to conclude this proof, it is sufficient to show that the graph $W_{p}^{n} \backslash M_{H}$ is connected. Note that each vertex of $W_{p}^{n}$ has degree $d=n-2 p+1$. Since $M_{H}$ induces a clique in $\bar{W}_{p}^{n}$, we have $\left|M_{H}\right| \leq p$. Actually, $M_{H}$ is contained in a maximal clique $K$ that comprises $p$ consecutive elements in the natural cyclic ordering of $\bar{W}_{p}^{n}$. Since every vertex in $K$ is adjacent, in $\bar{W}_{p}^{n}$, to a vertex of $\bar{W}_{p}^{n} \backslash K$, it suffices to consider the case where $M_{H}=K$. So, assume that $M_{H}$ has exactly $p$ elements (consecutive vertices of $\left.\bar{W}_{p}^{n}\right)$.

Let $v_{1}, v_{2} \in H$ be the predecessor and the successor, respectively, of the vertex set $M_{H}$ in the cyclic ordering. Thus, $v_{1}$ is adjacent, in the graph $W_{p}^{n}$, to exactly one vertex in $M_{H}$ and to $d-1$ vertices in $W_{p}^{n} \backslash M_{H}$. Analogously, $v_{2}$ is adjacent, in $W_{p}^{n}$, to one exactly vertex in $M_{H}$ and to the $d-1$ vertices in $W_{p}^{n} \backslash M_{H}$. Let $N_{1}$ and $N_{2}$ be the sets of neighbors of $v_{1}$ and $v_{2}$ in $W_{p}^{n} \backslash M_{H}$, respectively. The elements of $N_{1}$ are consecutive vertices succeeding $M_{H}$ whereas $N_{2}$ are consecutive vertices preceding $M_{H}$ (see Figure 9.3). Note that $v_{1} \in N_{2}$ and $v_{2} \in N_{1}$. Thus, $N=N_{1} \cup N_{2}$ induces a connected subgraph of $W_{p}^{n} \backslash M_{H}$.

We now prove that this subgraph is actually $W_{p}^{n} \backslash M_{H}$ by showing that $N \supseteq V\left(W_{p}^{n} \backslash M_{H}\right)$. We have $2(d-1)=2 n-4 p=(n-3 p)+(n-p) \geq n-p=\left|V\left(W_{p}^{n} \backslash M_{H}\right)\right|$, where the inequality is due to $\alpha\left(\bar{W}_{p}^{n}\right)=\left\lfloor\frac{n}{p}\right\rfloor \geq 3$, which implies $n \geq 3 p$. Therefore, since $N$ comprises $d-1$ vertices preceding $M_{H}$ and $d-1$ vertices succeeding $M_{H}$ in the cyclic order, we can conclude that $N \supseteq V\left(W_{p}^{n} \backslash M_{H}\right)$. 


\section{Part IV}

\section{Proper orientation}





\section{Chapter 10}

\section{The proper orientation problem}

In this chapter, we give the basic definitions and present some results from the literature related to the proper orientation problem. These are the subjects of the two first sections. In the third section we present an overview of the main results we obtained on this problem.

\subsection{Basic definitions}

An orientation of a graph $G$ is a digraph $D$ obtained from $G$ by replacing each edge by just one of the two possible arcs with the same endvertices. For each $v \in V(G)$, the in-degree of $v$ in $D$, denoted by $d_{D}^{-}(v)$, is the number of arcs with head $v$ in $D$. We use the notation $d^{-}(v)$ when the orientation $D$ is clear from the context. The orientation $D$ of $G$ is proper if $d^{-}(u) \neq d^{-}(v)$, for all $u v \in E(G)$. An orientation with maximum in-degree at most $k$ is called a $k$-orientation. The proper orientation number of a graph $G$, denoted by $\vec{\chi}(G)$, is the minimum integer $k$ such that $G$ admits a proper $k$-orientation. This graph parameter was introduced by Ahadi and Dehghan [3]. It is well-defined for any graph $G$ since one can always obtain a proper $\Delta(G)$-orientation using the following procedure. Consider a vertex, say $v$, of maximum degree in $G$, orient all edges incident to $v$ towards it, and repeat this procedure on $G-v$ if it is not an empty graph. Hence, it holds that $\vec{\chi}(G) \leq \Delta(G)$. Note that every proper orientation of a graph $G$ induces a proper vertex coloring of $G$. Furthermore, observe that, in any $k$-orientation of $G$, $d^{-}(v) \in\{0,1, \ldots, k\}$ for every $v \in V(G)$. Thus, it follows that $\vec{\chi}(G) \geq \chi(G)-1$. Therefore, we have the following sequence of inequalities:

$$
\omega(G)-1 \leq \chi(G)-1 \leq \vec{\chi}(G) \leq \Delta(G)
$$

These inequalities are best possible in the sense that, for a complete graph $K, \omega(K)-1=\chi(K)-1=$ $\vec{\chi}(K)=\Delta(K)$. However, one might expect better upper bounds on some parameter by taking a convex combination of two others. Reed [67] showed that there exists $\varepsilon_{0}>0$ such that $\chi(G) \leq \varepsilon_{0} \cdot \omega(G)+(1-$ $\left.\varepsilon_{0}\right) \Delta(G)$ for every graph $G$ and conjectured the following.

Conjecture 10.1 (Reed [67]). For every graph $G$,

$$
\chi(G) \leq\left\lceil\frac{\Delta(G)+1+\omega(G)}{2}\right\rceil .
$$

If true, this conjecture would be tight. Johannson [46] settled Conjecture 10.1 for $\omega(G)=2$ and $\Delta(G)$ sufficiently large. 
Likewise, one may wonder if similar upper bounds might be derived for the proper orientation number.

\section{Problem 10.2.}

(a) Does there exist a positive $\varepsilon_{1}$ such that $\vec{\chi}(G) \leq \varepsilon_{1} \cdot \omega(G)+\left(1-\varepsilon_{1}\right) \Delta(G)$ ?

(b) Does there exist a positive $\varepsilon_{2}$ such that $\vec{\chi}(G) \leq \varepsilon_{2} \cdot \chi(G)+\left(1-\varepsilon_{2}\right) \Delta(G)$ ?

Observe that both questions are intimately related. Indeed if the answer to (a) is positive for $\varepsilon_{1}$, then the answer to (b) is also positive for $\varepsilon_{1}$. On the other hand, if the answer to (b) is positive for $\varepsilon_{2}$, then the answer to (a) is also positive for $\varepsilon_{1}=\varepsilon_{0} \cdot \varepsilon_{2}$ by the above-mentioned result of Reed.

\subsection{Known results}

In their seminal paper [3], Ahadi and Dehghan focused on algorithmic aspects of the proper orientation number of regular graphs and planar graphs. They proved that it is $\mathcal{N} \mathcal{P}$-complete to decide whether $\vec{\chi}(G)=2$ for planar graphs $G$. Additionally, they showed that computing the proper orientation number of 4-regular graphs is $\mathcal{N} \mathcal{P}$-hard.

On the positive side, Ahadi and Dehghan [3] designed a polynomial-time algorithm for computing the proper orientation number of 3-regular graphs by reducing this problem to the problem of determining whether a given graph has chromatic number at most 2, which is known to be solvable in polynomial time. Furthermore, they proposed a simple algorithm with approximation ratio $\left(2-\frac{2}{r+2}\right)$ for the proper orientation problem on $r$-regular graphs.

The proper orientation problem has also been studied from a structural point of view. A cactus is a graph in which every 2-connected component is either an edge or a cycle, equivalently, it is a graph in which every edge is contained in at most one cycle. Very recently, Araujo et al. [7] showed that every cactus admits a proper orientation with maximum in-degree at most 7 . Additionally, they proved that the bound 7 is tight by showing a cactus having no proper orientation with maximum in-degree less than 7 .

A claw is a graph isomorphic to the complete bipartite graph $K_{1,3}$. A claw-free graph is a graph that does not have a claw as an induced subgraph. Araujo et al. [7] proved that if $G$ is a planar claw-free graph, then $\Delta(G) \leq 6$, which trivially implies $\vec{\chi}(G) \leq 6$. Furthermore, they showed a planar claw-free graph which has proper orientation at least 6 .

Also recently, Knox et al. [51] proved that 3-connected planar bipartite graphs have proper orientation number at most 6

\subsection{Contributions}

Our contributions on the proper orientation problem are twofold: algorithmic and structural. From the structural point of view, in Chapter 11, we answer Problem 10.2 positively in the case of bipartite graphs by showing that

$$
\text { if } G \text { is bipartite, then } \vec{\chi}(G) \leq\left\lfloor\frac{\Delta(G)+\sqrt{\Delta(G)}}{2}\right\rfloor+1 \text {. }
$$

We also present bipartite graphs $G$ such that $\vec{\chi}(G)=\Delta(G)=k$ for $k \in\{1,2,3\}$. In the case of trees, we show that $\vec{\chi}(T) \leq 4$ for every tree $T$. Moreover, we show that $\vec{\chi}(T) \leq 3$ if $\Delta(T) \leq 6$, and $\vec{\chi}(T) \leq 2$ if $\Delta(T) \leq 3$. We also argue that all these bounds are tight. 
From the algorithmic point of view, we study the computational complexity of computing the proper orientation number of bipartite and bounded degree graphs in Chapter 12. Ahadi and Dehghan [3] proved that it is $\mathcal{N} \mathcal{P}$-complete to decide whether $\vec{\chi}(G)=2$ for planar graphs $G$. We first improve their reduction and show that it is $\mathcal{N} \mathcal{P}$-complete to decide whether $\vec{\chi}(G) \leq 2$, for planar subcubic graphs $G$. In [3], Ahadi and Dehghan showed that it is $\mathcal{N} \mathcal{P}$-complete to decide whether $\vec{\chi}(G) \leq 3$ for 4-regular graphs $G$. Here, we show that, for every $k \geq 3$, it is $\mathcal{N} \mathcal{P}$-complete to decide whether $\vec{\chi}(G) \leq \Delta(G)-1$ for graphs $G$ with $\Delta(G)=k$. Finally, we show that it is also $\mathcal{N} \mathcal{P}$-complete to decide whether $\vec{\chi}(G) \leq 3$ for planar bipartite graphs $G$ with maximum degree 5 .

We remark that the results presented in this part of the thesis were obtained in collaboration with Júlio C. Araújo, Nathann Cohen, Susanna de Rezende and Frédéric Havet, and published in [6]. 


\section{Chapter 11}

\section{Upper bounds for bipartite graphs}

\subsection{General bipartite}

The aim of this section is to prove the following theorem.

Theorem 11.1. Let $G$ be a bipartite graph and let $k$ be a positive integer. If $\Delta(G)>2 k+\frac{\sqrt{1+8 k}+1}{2}$, then $\vec{\chi}(G) \leq \Delta(G)-k$.

In order to prove this theorem, we describe an algorithm (see Algorithm 3) that produces a proper $(\Delta(G)-$ $k$ )-orientation.

Let $G$ be a bipartite graph with bipartition $\{X, Y\}$ as in the statement of Theorem 11.1. The algorithm consists of two phases.

The first phase (lines 1 to 8 in Algorithm 3) produces an orientation, not necessarily proper, of the edges of $G$ in such a way that the in-degree of each vertex in $X$ is at most $k$ and the in-degree of each vertex in $Y$ is at most $\Delta(G)-k$. It proceeds as follows. We first orient all edges $x y \in E(G)$ from $x$ to $y$, where $x \in X$ and $y \in Y$. Then we define $k$ matchings as described subsequently.

Let $G_{1}=G$, and let $M_{1}$ be a matching in $G_{1}$ that covers all vertices of maximum degree. For each $i \in\{2, \ldots, k\}$, let $G_{i}$ be the graph obtained from $G_{i-1}$ by removing the edges in $M_{i-1}$, that is $G_{i}=G_{i-1}-M_{i-1}$, and let $M_{i}$ be a matching in $G_{i}$ that covers all vertices of degree $\Delta\left(G_{i}\right)$. Such an $M_{i}$ exists since it is well known that every bipartite graph $H$ has a proper $\Delta(H)$-edge-coloring. Clearly, we have $\Delta\left(G_{i}\right)=\Delta\left(G_{i-1}\right)-1$, for each $i \in\{2,3, \ldots, k\}$. Let $M:=\bigcup_{i=1}^{k} M_{i}$. Observe that if a vertex has degree $\Delta(G)-k+j$ in $G$, where $j \in\{1,2, \ldots, k\}$, then it is incident to at least $j$ edges in $M$. Hence, for all $j \in\{1,2, \ldots, k\}$ and for each vertex $y$ in $Y$ of degree $\Delta(G)-k+j$ in $G$, we reverse the orientation of exactly $j$ edges in $M$ incident to $y$. This ends the first phase.

The second phase reverses the orientation of some edges in $E(G) \backslash M$, step by step, in order to obtain a $(\Delta(G)-k)$-orientation. It remains to prove that this orientation is proper under the assumption of Theorem 11.1.

Before proving Theorem 11.1, let us introduce some notation and state a few properties of Algorithm 3. If $x \in X$ and $y \in Y$ are adjacent and have the same in-degree, then there exists a conflict between $x$ and $y$, and that $x$ (or $y$ ) conflicts. For each $x \in X$ and $\ell \in\{0,1, \ldots, \Delta(G)-1\}$, let $N_{\leq \ell}(x)=\{y \in$ $Y: x y \in E(G)$ and $\left.d^{-}(y) \leq \ell\right\}$ and, similarly, let $N_{=\ell}(x)=\left\{y \in Y: x y \in E(G)\right.$ and $\left.d^{-}(y)=\ell\right\}$.

Recall that the first phase of this algorithm corresponds to lines 1 to 8 and the second phase to lines 9 to 15 . The 'for' loop from line 10 to 15 is called the third loop. 
In what follows, for every pair $u, v$ of adjacent vertices in a directed graph, we say that $u$ is dominated by $v$, if there is an arc from $v$ to $u$.

Claim 11.2. At the end of the first phase of Algorithm 3, the following holds:

(a) $d^{-}(x) \leq k$ for all $x \in X$, and $d^{-}(y) \leq \Delta(G)-k$ for all $y \in Y$; and

(b) if $x \in X$ is dominated by some $y \in Y$, then $d^{-}(y)=\Delta(G)-k$.

Proof. Both statements follow directly from the reversal of the orientation of some edges in $M$ in the first phase.

In the second phase of the algorithm, we treat step by step the vertices $x \in X$ in such a way that, once they satisfy the condition of the 'while' loop (line 11), the reversal of the orientation of edges in line 14 ensures that:

(i) $x$ does not conflict at the end of the current step; and

(ii) $x$ will never conflict after the current step.

The main argument we use to prove the second fact is the following.

Claim 11.3. In the second phase of Algorithm 3, the in-degree of every vertex in $X$ never decreases and the in-degree of every vertex in $Y$ never increases.

Proof. This is straightforward, since all re-orientations are towards $X$.

Moreover, recall that, when decreasing the in-degree of vertices in $Y$ in the second phase of the algorithm, we only use vertices that have in-degree in $\{\Delta(G)-k-1, \ldots, 2\}$ at the end of the first phase.

Claim 11.4. During a given iteration $\ell$ of the third loop of Algorithm 3, only vertices belonging to $Y$ of in-degree at most $\ell$ have their in-degree decreased.

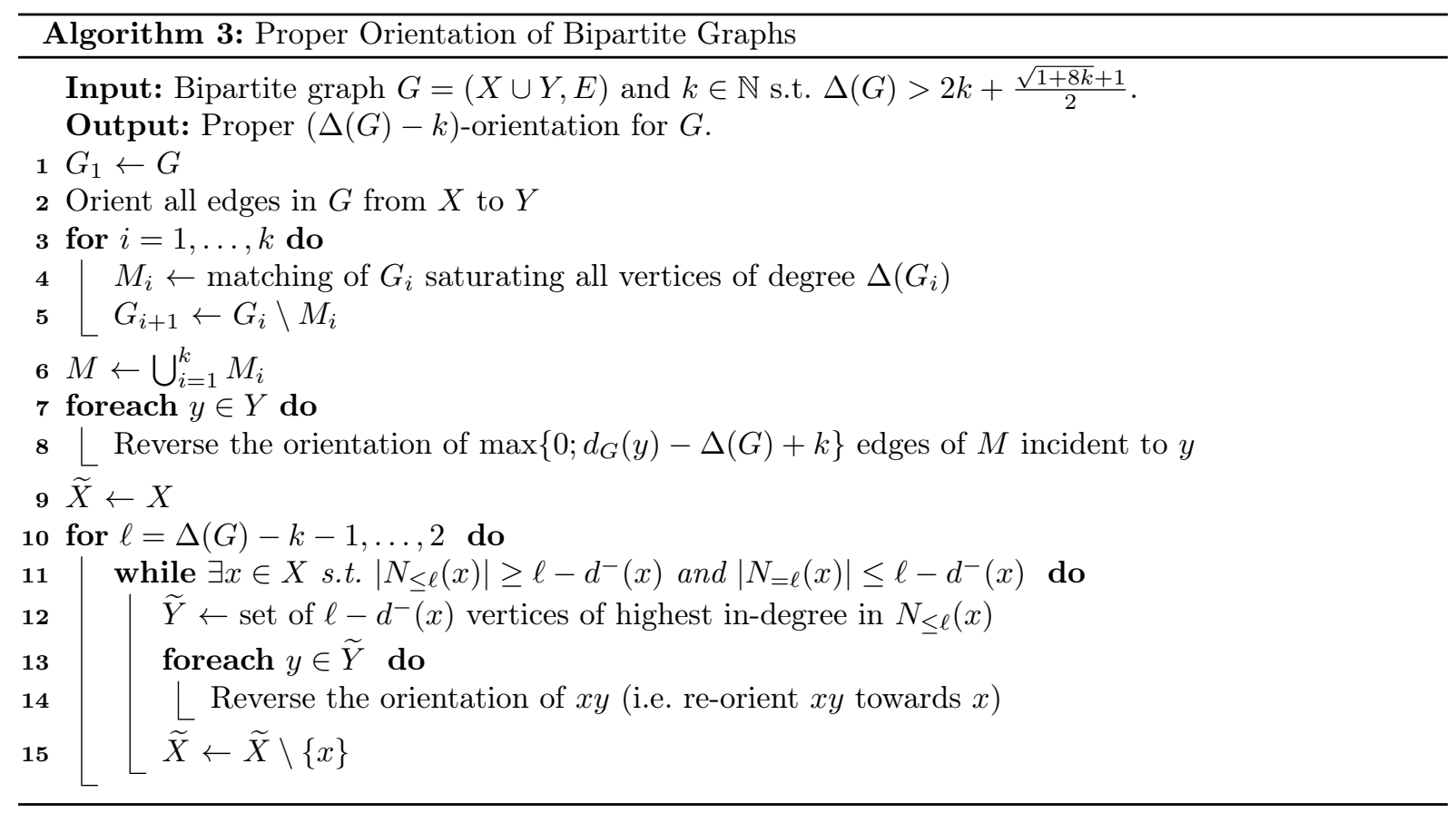


Proof. The only edges that are re-oriented are those incident to vertices of $N_{\leq \ell}(x)$, which, by definition, have in-degree at most $\ell$.

Using previous claims, we now prove that all vertices in $X$ that are removed from $\widetilde{X}$ do not conflict at the end of the execution of Algorithm 3.

Claim 11.5. Suppose that $x$ is removed from $\widetilde{X}$ during a given iteration $\ell$ of the third loop of Algorithm 3. At the end of the execution of the algorithm, the following hold:

(a) $d^{-}(x)=\ell ;$ and

(b) $x$ has no neighbors of in-degree $\ell$.

Proof. First note that, by Claims 11.2 and 11.4, every vertex in $\widetilde{X}$ is dominated only by vertices of in-degree $\Delta(G)-k$ at the beginning of the second phase. Therefore, at the beginning of iteration $\ell$ of the third loop, that is before re-orienting the edges incident to $x$, vertex $x$ dominates all vertices in $N_{\leq \ell}(x)$. Thus, after re-orienting $\ell-d^{-}(x)$ edges that are incident to $x$ and to vertices of $N_{\leq \ell}(x), x$ has in-degree exactly $\ell$. Note that, after $x$ is removed from $\tilde{X}$, the in-degree of $x$ does not change in the remainder of the second phase. Therefore, property (a) holds at the end of the execution of Algorithm 3.

Note also that, when $x$ is removed from $\tilde{X}$, it has at most $\ell-d^{-}(x)$ neighbors of in-degree $\ell$ (due to the condition of the 'while' loop in line 11 of Algorithm 3), and all of these vertices belong to $\tilde{Y}$. Thus, they have their in-degree decreased by 1 (lines 13 and 14). Therefore, at the end of this iteration of the 'while' loop, $x$ has no neighbor of in-degree $\ell$. By Claim 11.4 and since $\ell$ decreases throughout the algorithm, no vertex in $Y$ of in-degree strictly larger than $\ell$ has its in-degree decreased during or after this iteration. Moreover, by Claim 11.3, no vertex in $Y$ has its in-degree increased. Therefore, we conclude that, at the end of the execution of Algorithm 3, property (b) holds.

With those claims in hands, we are now ready to prove Theorem 11.1.

Proof of Theorem 11.1. Let $G$ be a bipartite graph with bipartition $\{X, Y\}$ such that $\Delta(G)>2 k+$ $\frac{\sqrt{1+8 k}+1}{2}$. We shall prove that Algorithm 3 returns a proper $(\Delta(G)-k)$-orientation of $G$. Note that the above inequality implies that $k<\frac{\Delta(G)}{2}$.

By Claim 11.5, at the end of the execution of Algorithm 3, all vertices in $X \backslash \widetilde{X}$ do not conflict. Thus, it suffices to show that the vertices in $\widetilde{X}$ do not conflict.

Suppose to the contrary that there exists $x^{*} \in \tilde{X}$ that conflicts at the end of the execution of Algorithm 3. Let $y^{*} \in Y$ be a neighbor of $x^{*}$ such that $d^{-}\left(y^{*}\right)=d^{-}\left(x^{*}\right)$ at the end of the execution.

Firstly, note that, at each iteration of the third loop, the condition of the 'while' loop in line 11 is not satisfied by $x^{*}$. Thus, during the execution of the second phase, the in-degree of $x^{*}$ does not change. Moreover, by Claims 11.2 and 11.3, we have $1 \leq d^{-}\left(x^{*}\right)=d^{-}\left(y^{*}\right) \leq k$ at the end of the execution.

We claim that if $x^{*}$ is dominated by some vertex $y \in Y$ at the end of the execution, then there is no conflict between $x^{*}$ and $y$. Indeed, no edge is re-oriented towards $x^{*}$ in the second phase, so $y$ already dominates $x$ at the end of the first phase. By Claim 11.2, $y$ has in-degree $\Delta(G)-k$ at the end of the first phase. Observe that the in-degree of $y$ does not decrease during the second phase because the third loop starts with the value $\Delta(G)-k-1$ and, by Claim 11.4, only vertices in $Y$ with degree at most this value have their in-degrees decreased. Since $k<\frac{\Delta(G)}{2}$, there is no conflict between $y$ and $x^{*}$. Thus, during all of the second phase, $x^{*}$ is dominated by $d^{-}(x)$ vertices of in-degree $\Delta(G)-k$ and dominates its remaining neighbors, among which is $y^{*}$.

We now use the fact that the 'while' condition is never satisfied by $x^{*}$ to get the contradiction that $\Delta(G) \leq 2 k+\frac{\sqrt{1+8 k}+1}{2}$. By Claim 11.4, vertex $y^{*}$ must have its in-degree set to $d^{-}\left(x^{*}\right)$ no later than 
iteration $\ell_{1}:=d^{-}\left(x^{*}\right)+1$ in the third loop. Thus, at the end of iteration $\ell_{1},\left|N_{\leq \ell_{1}}\left(x^{*}\right)\right| \geq \ell_{1}-d^{-}\left(x^{*}\right)=1$ holds since $y^{*} \in N_{\leq \ell_{1}}\left(x^{*}\right)$. Since the 'while' condition is never satisfied by $x^{*}$, we deduce that $\left|N_{=\ell_{1}}\left(x^{*}\right)\right|>$ $\ell_{1}-d^{-}\left(x^{*}\right)=1$. Hence, $x^{*}$ has at least two outneighbors, say $y_{1}$ and $y_{2}$, of in-degree exactly $\ell_{1}$. Note that, by Claim 11.4, $y_{1}$ and $y_{2}$ have in-degree $\ell_{1}$ at the end of the execution of the algorithm. In addition, these vertices are distinct from $y^{*}$.

During iteration $\ell_{1}$, by Claim 11.4, only vertices of in-degree at most $\ell_{1}$ have their in-degrees decreased. Thus, at the end of iteration $\ell_{2}:=d^{-}\left(x^{*}\right)+2, y_{1}$ and $y_{2}$ already have in-degree exactly $\ell_{1}$, so we have $\left|N_{\leq \ell_{2}}\left(x^{*}\right)\right| \geq \ell_{2}-d^{-}\left(x^{*}\right)=2$. Since the 'while' condition is not satisfied by $x^{*}$, it has at least three outneighbors of in-degree exactly $\ell_{2}$ at the end of iteration $\ell_{2}$, that is $\left|N_{=\ell_{2}}\left(x^{*}\right)\right|>2$. Moreover, those vertices have in-degree $\ell_{2}$ at the end of the execution of the algorithm. In the same way, for all $i \in\left\{3,4, \ldots, \Delta(G)-k-d^{-}\left(x^{*}\right)-1\right\}$, setting $\ell_{i}=d^{-}\left(x^{*}\right)+i$, we show that $x^{*}$ has at least $i+1$ outneighbors of in-degree exactly $\ell_{i}$ at the end of iteration $\ell_{i}$ of the third loop, and thus at the end of the execution of Algorithm 3.

Hence, $x^{*}$ dominates at least $\xi:=\sum_{i=0}^{\Delta(G)-k-d^{-}\left(x^{*}\right)-1}(i+1)$ vertices. However, $x^{*}$ has at most $\Delta(G)$ neighbors, so

$$
\xi=\frac{\left(\Delta(G)-k-d^{-}\left(x^{*}\right)\right)\left(\Delta(G)-k-d^{-}\left(x^{*}\right)+1\right)}{2} \leq \Delta(G)-d^{-}\left(x^{*}\right) .
$$

Solving this inequality, we obtain $\Delta(G) \leq k+d^{-}\left(x^{*}\right)+\frac{\sqrt{1+8 k}+1}{2}$. Since $d^{-}\left(x^{*}\right) \leq k$, we conclude that $\Delta(G) \leq 2 k+\frac{\sqrt{1+8 k}+1}{2}$, which contradicts the hypothesis of the theorem. Therefore, no vertex in $\widetilde{X}$ conflicts and thus the orientation produced by Algorithm 3 is a proper $(\Delta(G)-k)$-orientation for $G$.

Theorem 11.6. If $G$ is a bipartite graph, then

$$
\vec{\chi}(G) \leq\left\lfloor\frac{\Delta(G)+\sqrt{\Delta(G)}}{2}\right\rfloor+1
$$

Proof. By Theorem 11.1, for every $k \in \mathbb{N}$, if $\Delta(G)>2 k+\frac{\sqrt{1+8 k}+1}{2}$, then $\vec{\chi}(G) \leq \Delta(G)-k$. In order to obtain a good upper bound for $\vec{\chi}(G)$, we must find the largest positive integer $k$ such that the condition of Theorem 11.1 holds for a given graph $G$.

Solving the inequality for $k$, we obtain that $k<\frac{\Delta(G)-\sqrt{\Delta(G)}}{2}$. Since $k$ is integer, the condition holds when

$$
k \leq\left\lceil\frac{\Delta(G)-\sqrt{\Delta(G)}}{2}\right\rceil-1
$$

Therefore, we conclude that

$$
\vec{\chi}(G) \leq \Delta(G)-\left\lceil\frac{\Delta(G)-\sqrt{\Delta(G)}}{2}\right\rceil+1=\left\lfloor\frac{\Delta(G)+\sqrt{\Delta(G)}}{2}\right\rfloor+1
$$

Note that if $G$ is bipartite and $\Delta(G) \in\{2,3,4\}$, then the bound of Theorem 11.6 is equal to the trivial upper bound $\vec{\chi}(G) \leq \Delta(G)$. For $\Delta(G)=1$ and $\Delta(G)=2$, this bound is tight due to the paths with 2 and 4 vertices, respectively. In the sequel, we present a tight example for the case $\Delta(G)=3$.

Proposition 11.7. There exists a bipartite graph $G$ with $\Delta(G)=3$ and $\vec{\chi}(G)=3$. 


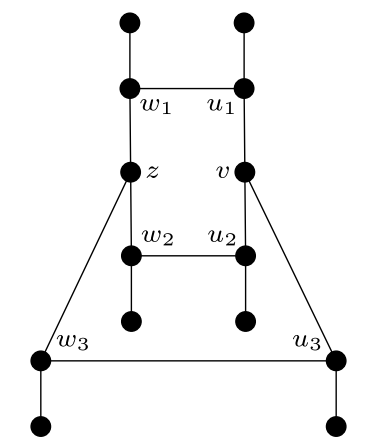

Figure 11.1: $A$ bipartite graph $B_{3}$ for which $\vec{\chi}\left(B_{3}\right)=\Delta\left(B_{3}\right)=3$.

Proof. Consider the bipartite graph $B_{3}$ depicted in Figure 11.1. Suppose to the contrary that there exists a proper 2-orientation $D$ of $B_{3}$. Observe that, if a vertex $x$ has a neighbor of degree 1 , say $y$, then, in any proper orientation, $x$ has in-degree 1 only if $y$ dominates $x$.

If $d^{-}(v)=0$, then $v$ dominates $u_{i}$, for all $i \in\{1,2,3\}$. Note that $d^{-}\left(u_{i}\right) \neq 1$ for all $i \in\{1,2,3\}$ due to their neighbors of degree 1 . Thus, we obtain $d^{-}\left(u_{i}\right)=2$, for each $i \in\{1,2,3\}$. Moreover, $w_{i}$ dominates $z$ and $u_{i}$ for all $i \in\{1,2,3\}$, because $d^{-}\left(w_{i}\right) \neq 2$ and $w_{i}$ has a neighbor of degree 1 . Therefore, we have $d^{-}(z)=3$, a contradiction.

If $d^{-}(v)=1$, then w.l.o.g. $v$ is dominated by $u_{3}$, and dominates $u_{1}$ and $u_{2}$. Similarly to the previous case, we have that $d^{-}\left(u_{1}\right)=d^{-}\left(u_{2}\right)=2$ and $z$ is dominated by $w_{1}$ and $w_{2}$. Thus, $d^{-}(z)=2$ and $z$ dominates $w_{3}$, a contradiction, since $w_{3}$ has a neighbor of degree 1 and $d^{-}\left(w_{3}\right) \neq 2$.

Finally, if $d^{-}(v)=2$, then $v$ dominates $u_{i}$ for some $i \in\{1,2,3\}$. We again arrive to a contradiction, since $u_{i}$ has a neighbor of degree 1 and $d^{-}\left(u_{i}\right) \neq 2$.

Therefore, there exists no proper 2-orientation of $B_{3}$. Since $\Delta\left(B_{3}\right)=3$, we conclude that $\vec{\chi}\left(B_{3}\right)=$ 3.

Ahadi and Dehghan [3] proved that, for every $r \in \mathbb{N}$, if $G$ is a bipartite $r$-regular graph, then $\vec{\chi}(G)=$ $\left\lceil\frac{r+1}{2}\right\rceil$. In this sense, we next show an upper bound tighter than the one of Theorem 11.6 for bipartite graphs whose minimum degree is very close to its maximum degree.

Proposition 11.8. If $G$ is a bipartite graph, then

$$
\vec{\chi}(G) \leq \Delta(G)-\left\lfloor\frac{\delta(G)-1}{2}\right\rfloor .
$$

Proof. Let $\{X, Y\}$ be a bipartition of $G$ and let $k=\left\lfloor\frac{\delta(G)-1}{2}\right\rfloor$. The following steps correspond exactly to the first phase of Algorithm 3.

Consider the orientation of $G$ defined as follows. We first orient all edges $x y \in E(G)$ from $x$ to $y$, where $x \in X$ and $y \in Y$. Then, we define $k$ matchings as described subsequently. Let $G_{1}=G$, and let $M_{1}$ be a matching in $G_{1}$ that covers all vertices of maximum degree. For each $i \in\{2, \ldots, k\}$, let $G_{i}=$ $G_{i-1}-M_{i-1}$, and let $M_{i}$ be a matching in $G_{i}$ that covers all vertices of degree $\Delta\left(G_{i}\right)$. Clearly, we have $\Delta\left(G_{i}\right)=\Delta\left(G_{i-1}\right)-1$, for each $i \in\{2,3, \ldots, k\}$. Let $M:=\bigcup_{i=1}^{k} M_{i}$. Observe that if a vertex has degree $\Delta(G)-k+j$ in $G$, where $j \in\{1,2, \ldots, k\}$, then it is incident to at least $j$ edges in $M$. Hence, for all $j \in\{1,2, \ldots, k\}$ and for each vertex $y$ in $Y$ of degree $\Delta(G)-k+j$ in $G$, we reverse the orientation of exactly $j$ edges in $M$ incident to $y$.

In this orientation of $G$, every vertex of $X$ has in-degree at most $k$, and every vertex of $Y$ has in-degree 
at least $\delta(G)-k$, which is larger than $k$, and at most $\Delta(G)-k$. Therefore, we have a proper $(\Delta(G)-k)$ orientation of $G$.

\subsection{Trees}

The main goal of this section is to prove the following theorem.

Theorem 11.9. If $T$ is a tree, then the following statements hold:

(1) if $\Delta(T) \leq 3$, then $\vec{\chi}(T) \leq 2$;

(2) if $\Delta(T) \leq 6$, then $\vec{\chi}(T) \leq 3$;

(3) $\vec{\chi}(T) \leq 4$.

Before proving Theorem 11.9, we show that the three statements of the theorem are tight in the following sense: there is a tree with maximum degree 4 and proper orientation number 3 , and a tree with maximum degree 7 and proper orientation number 4 .

Let $R_{2}$ and $R_{3}$ be the trees with root $x$ depicted in Figures 11.2(a) and 11.2(b), respectively.

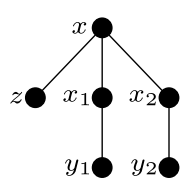

(a) Tree $R_{2}$ with root $x$.

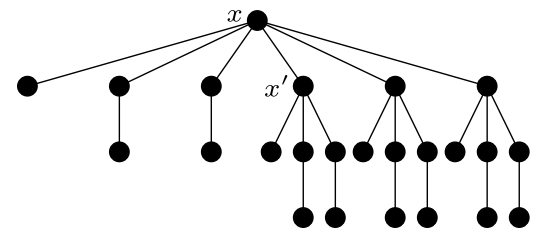

(b) Tree $R_{3}$ with root $x$

Figure 11.2: Rooted trees $R_{2}$ and $R_{3}$.

Let $H$ be an induced proper subgraph of $G$ and let $x \in V(H)$. The subgraph $H$ is $x$-pendant if there exist no edges between $V(H) \backslash\{x\}$ and $V(G) \backslash V(H)$.

Lemma 11.10. Let $G$ be a graph containing an $x$-pendant $R_{2}$ and let $D$ be a proper orientation of $G$.

(i) If $d^{-}(x)=1$, then $x$ is dominated by $z$.

(ii) If $d^{-}(x)=2$, then $x$ is dominated by $x_{1}$ and $x_{2}$.

Moreover, if $d^{-}(x) \in\{1,2\}$, then $x$ dominates all its neighbors that are not in $R_{2}$.

Proof. (i) If $d^{-}(x)=1$, then $d^{-}(z) \neq 1$ because $D$ is proper. Therefore, we have $d^{-}(z)=0$, and thus $z$ dominates $x$.

(ii) Suppose that $d^{-}(x)=2$ and $x$ dominates $x_{i}$, for some $i \in\{1,2\}$. Thus, $d^{-}\left(x_{i}\right) \geq 1$ and, since $D$ is proper, we deduce that $d^{-}\left(x_{i}\right)=1$. It follows that $x_{i}$ dominates $y_{i}$, so $d^{-}\left(y_{i}\right)=1$, which is a contradiction since $D$ is proper. Therefore, $x$ is dominated by $x_{1}$ and $x_{2}$.

Corollary 11.11. Let $T_{3}$ be the tree obtained from two copies of $R_{2}$ with roots $x$ and $\hat{x}$ by adding the edge $x \hat{x}$. It follows that $\vec{\chi}\left(T_{3}\right)=3$.

Lemma 11.12. Let $G$ be a graph with an $x$-pendant $R_{3}$. In any proper 3-orientation of $G, x$ dominates all its neighbors in $G-R_{3}$. 
Proof. Suppose to the contrary that there exists a proper 3-orientation $D$ of $G$ in which a vertex $y \in$ $V\left(G-R_{3}\right)$ dominates $x$. It follows that $d^{-}(x) \geq 1$. By Lemma 11.10 , we obtain $d^{-}(x)=3$. Moreover, $x$ dominates at least one of its neighbors of degree 4 in $R_{3}$, say $x^{\prime}$. Since $D$ is proper, $d^{-}\left(x^{\prime}\right) \in\{1,2\}$. Therefore, the subtree $R_{2}$ rooted at $x^{\prime}$ contradicts Lemma 11.10.

Corollary 11.13. Let $T_{4}$ be the tree obtained from two copies of $R_{3}$ with roots $x$ and $\hat{x}$ by adding the edge $x \hat{x}$. It follows that $\vec{\chi}\left(T_{4}\right)=4$.

In order to prove Theorem 11.9, we need a few definitions. In a tree $T$, a vertex $x$ is a twig-vertex if it is not a leaf and all its neighbors except possibly one are leaves. A twig is a subtree induced by a twig-vertex and its adjacent leaves. The twig rooted at $x$ is the twig that contains the twig-vertex $x$.

A vertex $x$ of $T$ is a bough-vertex if it is neither a leaf, nor a twig-vertex, and all its neighbors except possibly one are twig-vertices or leaves. A bough is a subtree induced by a bough-vertex $x$, the leaves adjacent to $x$, and the vertices of the twigs whose roots are adjacent to $x$. The bough rooted at $x$ is the bough that contains the bough-vertex $x$.

A vertex $x$ of $T$ is a branch-vertex if it is neither a leaf, nor a twig-vertex, nor a bough-vertex, and all its neighbors except possibly one are bough-vertices, twig-vertices or leaves.

We now proceed to prove Theorem 11.9.

Proof of Theorem 11.9. We prove the three statements by using similar arguments. For $i \in\{1,2,3\}$, we consider a minimal counter-example $M_{i}$ to statement $(i)$ with respect to the number of vertices, and derive a contradiction that implies that no counter-example exists. Since $M_{i}$ is a minimal counter-example, we have $\vec{\chi}\left(M_{i}\right)>i+1$, but $\vec{\chi}(T) \leq i+1$, for any proper subtree $T$ of $M_{i}$. We use the latter fact to derive a proper $(i+1)$-orientation of $M_{i}$, which contradicts $\vec{\chi}\left(M_{i}\right)>i+1$.

Claim 11.14. For every $i \in\{1,2,3\}$, every vertex of $M_{i}$ is adjacent to at most one leaf.

Proof. Suppose to the contrary that a vertex $v$ is adjacent to two leaves, $u$ and $w$. Let $D$ be a proper $(i+1)$ orientation of $M_{i}-u$. In case $d_{D}^{-}(v) \neq 1$, one can obtain a proper $(i+1)$-orientation of $M_{i}$ from $D$ by orienting $v u$ from $v$ to $u$. Consequently, we have $d_{D}^{-}(v)=1$. Thus, $v w$ is oriented from $w$ to $v$, because $D$ is proper. Hence, $v$ dominates all its neighbors except $w$ in $D$, so $d_{D}^{-}(y) \geq 2$ for all $y \in N_{M_{i}}(v) \backslash\{u, w\}$. In particular, $u$ and $w$ are the only leaves adjacent to $v$. Therefore, one can obtain a proper $(i+1)$-orientation of $M_{i}$ from $D$ by reversing the orientation of $v w$ and orienting $v u$ from $v$ to $u$, a contradiction.

Claim 11.15. For every $i \in\{1,2,3\}$, every twig-vertex of $M_{i}$ is adjacent to exactly one leaf.

Proof. It follows directly from the definition of twig-vertex and Claim 11.14.

Claim 11.16. For every $i \in\{1,2,3\}$, every vertex of $M_{i}$ is adjacent to at most two twig-vertices.

Proof. Suppose to the contrary that a vertex $v$ is adjacent to $p$ twig-vertices $t_{1}, \ldots, t_{p}$ with $p \geq 3$. By Claim $11.15, t_{j}$ is adjacent to a single leaf $u_{j}$, for every $j \in\{1, \ldots, p\}$. By minimality of $M_{i}$, the tree $T=M_{i}-\bigcup_{j=3}^{p}\left\{t_{j}, u_{j}\right\}$ has a proper $(i+1)$-orientation $D$. If $d_{D}^{-}(v) \neq 2$, then $D$ can be extended to a proper $(i+1)$-orientation of $M_{i}$ by orienting the edges $v t_{j}$ and $u_{j} t_{j}$ towards $t_{j}$, for all $j \in\{3, \ldots, p\}$. Hence, we have $d_{D}^{-}(v)=2$, and so $v$ must be dominated by $t_{1}$ and $t_{2}$ since $D$ is proper. Thus, every vertex $y \in N_{T}(v) \backslash\left\{t_{1}, t_{2}\right\}$ satisfies $d_{D}^{-}(y) \geq 1$. Therefore, $D$ can be extended to a proper $(i+1)$-orientation of $M_{i}$ by orienting all edges $v t_{j}$ and $t_{j} u_{j}$ towards $t_{j}$, for every $j \in\{1, \ldots, p\}$, a contradiction.

Claim 11.17. For every $i \in\{1,2,3\}$, every bough-vertex of $M_{i}$ is adjacent to exactly one leaf and exactly two twig-vertices. In other words, every bough is isomorphic to $R_{2}$. 
Proof. Let $i \in\{1,2,3\}$ and let $v$ be a bough-vertex of $M_{i}$. By definition of bough-vertex, $v$ has at most one neighbor that is neither a twig nor a leaf. Let us denote this vertex by $r$ if it exists. Let $t_{1}, \ldots, t_{p}$ be the twig-vertices adjacent to $v$. By definition of bough-vertex and Claim 11.16, we have $p \in\{1,2\}$. Furthermore, by Claim 11.15, $t_{j}$ is adjacent to a single leaf $u_{j}$, for all $j \in\{1, \ldots, p\}$.

By Claim 11.14, vertex $v$ is adjacent to at most one leaf. Suppose to the contrary that $v$ is adjacent to no leaves. Thus, $N_{M_{i}}(v)=\left\{t_{1}, \ldots, t_{p}, r\right\}$. Let $T=M_{i}-\bigcup_{j=1}^{p}\left\{t_{j}, u_{j}\right\}$. By the minimality of $M_{i}, T$ has a proper $(i+1)$-orientation $D$. Note that $d_{D}^{-}(v) \leq d_{T}(v) \leq 1$. Consequently, one can obtain a proper $(i+1)$-orientation of $M_{i}$ by orienting the edges $v t_{j}$ and $t_{j} u_{j}$ towards $t_{j}$, for every $j \in\{1, \ldots, p\}$, a contradiction. Hence, $v$ is adjacent to exactly one leaf $w$.

Suppose to the contrary that $p=1$. Let $D$ be a proper $(i+1)$-orientation of $M_{i}-\left\{t_{1}, u_{1}\right\}$. If $d_{D}^{-}(v) \neq 2$, $D$ can be extended to a proper $(i+1)$-orientation of $M_{i}$ by orienting $v t_{1}$ and $t_{1} u_{1}$ towards $t_{1}$. It follows that $d_{D}^{-}(v)=2$. Thus, the vertex $r$ exists and $r v$ and $v w$ are oriented towards $v$. In this case, $D$ can be extended to a proper $(i+1)$-orientation of $M_{i}$ by reversing the orientation of $v w$, orienting $v t_{1}$ towards $v$, and $t_{1} u_{1}$ towards $u_{1}$, a contradiction. Therefore, we conclude that $p=2$.

We are now ready to prove (1). By Claim 11.17, the tree $M_{1}$ has neither branch-vertices nor two adjacent bough-vertices since $\Delta\left(M_{1}\right) \leq 3$. Consequently, by Claims 11.15 and 11.17 , the tree $M_{1}$ is either the tree $R_{2}$ depicted in Figure 11.2(a), or a path on at most four vertices. It is a simple matter to check that these trees have proper orientation number at most 2 . Hence, $\vec{\chi}\left(M_{1}\right) \leq 2$, a contradiction. This proves (1).

An orientation of a tree is antidirected if every vertex has in-degree 0 or out-degree 0 . There are two kinds of antidirected orientations of a rooted tree, one in which the root has in-degree 0 , called out-antidirected, and one in which the root has out-degree 0, called in-antidirected. An orientation of a bough in $M_{i}$ is nice if it is obtained from an in-antidirected orientation by reversing the arc between the root and its adjacent leaf.

Claim 11.18. For every $i \in\{2,3\}$, every vertex $v$ of $M_{i}$ is adjacent to at most three bough-vertices.

Proof. The argument is similar to the one in Claim 11.16. Suppose to the contrary that $v$ is adjacent to $p \geq 4$ bough-vertices $b_{j}, j \in\{1, \ldots, p\}$. For $j \in\{1, \ldots, p\}$, let $B_{j}$ be the bough rooted at $b_{j}$, and let $T=M_{i}-\bigcup_{j=4}^{p} B_{j}$. By the minimality of $M_{i}, T$ has a proper $(i+1)$-orientation $D$.

If $d_{D}^{-}(v) \neq 3$, then $D$ can be extended to a proper $(i+1)$-orientation of $M_{i}$ by taking the nice orientation of $B_{j}$ and orienting $v b_{j}$ towards $b_{j}$ for all $j \in\{4, \ldots, p\}$. This orientation is proper because the in-degrees of the vertices of $D$ are unchanged and $d_{D}^{-}\left(b_{j}\right)=3$ for all $j \in\{4, \ldots, p\}$. Thus, we have $d_{D}^{-}(v)=3$. Since $D$ is proper, vertex $v$ is dominated by $b_{1}, b_{2}$ and $b_{3}$ in $D$, and so it dominates all its neighbors that are not bough-vertices. Thus, one can obtain a proper $(i+1)$-orientation of $M_{i}$ by taking the nice orientation of $B_{j}$ and (re)-orienting $v b_{j}$ towards $b_{j}$ for all $j \in\{1, \ldots, p\}$. This orientation is proper because the in-degrees of the vertices of $V(T) \backslash\left(\bigcup_{j=1}^{3} V\left(B_{j}\right) \cup\{v\}\right)$ are not changed, the orientation of $B_{j}$ for $j \in\{1, \ldots, p\}$ is proper, $d^{-}(v)=0$ and all neighbors of $v$ are dominated by $v$, and so they have in-degree at least 1 .

Claim 11.19. For every $i \in\{2,3\}$, every branch-vertex is adjacent to exactly one leaf, two twig-vertices and three bough-vertices. In other words, every branch is isomorphic to $R_{3}$.

Proof. The arguments are similar to the ones presented in Claim 11.17.

Let $i \in\{2,3\}$ and let $v$ be a branch-vertex of $M_{i}$. By definition of branch-vertex, $v$ has at most one neighbor that is neither a bough, nor a twig, nor a leaf. Let us denote this vertex by $r$ if it exists. 
Let $b_{1}, \ldots, b_{p}$ be the bough-vertices that are adjacent to $v$, and $t_{1}, \ldots, t_{q}$ be the twig-vertices adjacent to $v$. By definition of branch-vertex and Claim 11.18 we have $p \in\{1,2,3\}$, and by Claim 11.16 we have $q \in\{0,1,2\}$. For $j \in\{1, \ldots, p\}$, let $B_{j}$ be the bough rooted at $b_{j}$. By Claim 11.15, vertex $t_{k}$ is adjacent to a single leaf $u_{k}$, for $k \in\{1, \ldots, q\}$.

Let $T=M_{i}-\left(\bigcup_{j=1}^{p} V\left(B_{j}\right) \cup \bigcup_{k=1}^{q}\left\{t_{k}, u_{k}\right\}\right)$. By minimality of $M_{i}$, the tree $T$ admits a proper $(i+1)$ orientation $D$. By Claim 11.14, $v$ is adjacent to at most one leaf. Suppose to the contrary that $v$ has no leaves in its neighborhood. Thus, $d_{D}^{-}(v) \leq d_{T}(v) \leq 1$. It follows that $D$ can be extended to a proper $(i+1)$-orientation of $M_{i}$ by taking the nice orientation of $B_{j}$ and orienting the edge $v b_{j}$ towards $b_{j}$, for every $j \in\{1, \ldots, p\}$, and orienting the edges $v t_{k}$ and $t_{k} u_{k}$ towards $t_{k}$, for every $k \in\{1, \ldots, q\}$. This orientation is indeed proper since $d^{-}\left(b_{j}\right)=3$, for all $j \in\{1, \ldots, p\}$, and $d^{-}\left(t_{k}\right)=2$, for all $k \in\{1, \ldots, q\}$. Therefore, $v$ is adjacent to a single leaf $w$.

Suppose to the contrary that $q \leq 1$. Thus, we have $d_{D}^{-}(v) \leq d_{T}(v) \leq 2$. If $d_{D}^{-}(v) \neq 2, D$ can be extended to a proper $(i+1)$-orientation of $M_{i}$ as above, that is by taking the nice orientation of $B_{j}$ and orienting the edges $v b_{j}$ towards $b_{j}$ for $j \in\{1, \ldots, p\}$, and orienting the edges $v t_{1}$ and $t_{1} u_{1}$ towards $t_{1}$ if $q=1$. It follows that $d_{D}^{-}(v)=2$, and both $r v$ and $w v$ are oriented towards $v$. Thus, one can obtain a proper $(i+1)$-orientation of $M_{i}$ as follows. We use the nice orientation of $B_{j}$ and orient the edges $v b_{j}$ towards $b_{j}$, for each $j \in\{1, \ldots, p\}$. In addition, we revert the orientation of $v w$, and orient $v t_{1}$ and $t_{1} u_{1}$ away from $t_{1}$ if $q=1$. This orientation is indeed proper because the degree of every vertex in $T-w$ is not changed, $d_{D}^{-}(w) \in\{0,1\}, d_{D}^{-}\left(b_{j}\right)=3$ for all $j \in\{1, \ldots, p\}$, and $d_{D}^{-}\left(t_{1}\right)=0$ if $q=1$. Therefore, we obtain $q=2$.

Suppose to the contrary that $p \leq 2$. Let $T^{\prime}=M_{i}-\bigcup_{j=1}^{p} B_{j}$. By minimality of $M_{i}, T^{\prime}$ admits a proper $(i+1)$-orientation $D^{\prime}$. If $d_{D^{\prime}}^{-}(v) \neq 3$, then by taking the nice orientation of $B_{j}$ and orienting the edge $v b_{j}$ towards $b_{j}$ for $j \in\{1, \ldots, p\}$, we obtain a proper $(i+1)$-orientation of $M_{i}$. If $d_{D^{\prime}}^{-}(v)=3$, then at least two vertices $v_{1}$ and $v_{2}$ in $\left\{w, t_{1}, t_{2}\right\}$ dominate $v$ in $D^{\prime}$. We obtain a proper $(i+1)$-orientation of $M_{i}$ by orienting the arcs incident to $p$ vertices in $\left\{v_{1}, v_{2}\right\}$ so that these vertices obtain out-degree 0 , orienting all edges $v b_{j}$ towards $v$ and taking the out-antidirected orientation of $B_{j}$ for every $j \in\{1, \ldots, p\}$. Therefore, we conclude that $p=3$ by Claim 11.18 .

Claim 11.20. For every $i \in\{2,3\}, M_{i}$ has a branch-vertex.

Proof. Suppose to the contrary that $M_{i}$ has no branch-vertex. By Claims 11.17 and $11.15, M_{i}$ is either $T_{3}$ (as defined in Corollary 11.11), or $R_{2}$, or a path on at most 4 vertices. By Corollary 11.11, $\vec{\chi}\left(T_{3}\right)=3$, and it is easy to check that $R_{2}$ and paths on at most four vertices have proper orientation number at most 2, a contradiction.

By Claim 11.20, $M_{2}$ has a branch-vertex. By Claim 11.19 and since $\Delta\left(M_{2}\right) \leq 6$, we deduce that $M_{2}$ is isomorphic to $R_{3}$. One can check that $\vec{\chi}\left(R_{3}\right)=3$, a contradiction. This proves (2).

By Claim 11.20, $M_{3}$ has a branch-vertex, say $v$. By Claim 11.19, $v$ is adjacent to three boughvertices $b_{1}, b_{2}, b_{3}$. Let $B_{j}$ be the bough rooted at $b_{j}$ for $j \in\{1,2,3\}$, and let $T=M_{3}-\left(B_{1} \cup B_{2} \cup B_{3}\right)$. By minimality of $M_{3}, T$ has a proper 4-orientation $D$. If $d_{D}^{-}(v) \neq 3$, then $D$ can be extended to a proper 4-orientation of $M_{3}$ by taking the nice orientation of $B_{j}$ and orienting the edge $v b_{j}$ towards $b_{j}$, for every $j \in\{1,2,3\}$. If $d_{D}^{-}(v)=3$, then $D$ can be extended to a proper 4-orientation of $M_{3}$ by taking the in-antidirected orientation of $B_{j}$ and orienting the edge $v b_{j}$ towards $b_{j}$, for every $j \in\{1,2,3\}$. This proves $(3)$.

We now show a class of trees that have proper orientation number 2. A tree is called even if the distance between any two leaves is even. 
Proposition 11.21. If $T$ is an even tree, then $\vec{\chi}(T) \leq 2$.

Proof. Let $r$ be the single neighbor of a leaf of $T$. Note that all leaves are at odd distance from $r$. We build a proper orientation $D$ of $T$ with maximum in-degree at most 2 as follows:

- Orient all edges from $r$ towards the leaves of $T$, in other words, orient the edges so that $d_{D}^{-}(r)=0$ and $d_{D}^{-}(v)=1$, for every $v \in V(T) \backslash\{r\}$.

- For each vertex at even distance from $r$ ( $r$ excluded), reverse one of its outgoing edges. This is possible since none of these vertices are leaves.

Observe that the vertices with in-degree 2 are exactly those at even distance from $r$ ( $r$ excluded). Since $r$ has in-degree 0 and its neighbors have in-degree 1, the obtained orientation is proper.

A referee pointed out a class of trees having proper orientation number at most 3.

Proposition 11.22. If $T$ is a tree having no vertex of degree 2, then $\vec{\chi}(T) \leq 3$.

Proof. Suppose that $|V(T)|=n$ and choose an arbitrary vertex $r \in V(T)$ to be its root. For every vertex $v \in V(T)$, let $\ell(v)$ denote the distance between $r$ and $v$ in $T$. Let $p(v)$ denote the neighbor of $v$ on the $r v$-path, i.e. its parent. Let $C(v)=N(v) \backslash\{p(v)\}$ denote the set of children of $v$.

Now we define a proper 3 -orientation of $T$ as follows. Take an ordering $v_{1}, \ldots, v_{n}$ of $V(T)$ in nondecreasing order according to $\ell$ (i.e. if $1 \leq i \leq j \leq n$, then $\ell\left(v_{i}\right) \leq \ell\left(v_{j}\right)$ ).

At step $i$, we orient all edges incident to $v_{i}$ that were not yet oriented. Thus, note that if $v_{i}$ is a leaf, then we just skip it, since the only edge $p\left(v_{i}\right) v_{i}$ incident to $v_{i}$ will be already oriented.

At step 1, we orient all edges $r w$ towards $w$, for every $w \in C(r)$. At step $i \in\{2, \ldots, n\}$, if $p\left(v_{i}\right) v_{i}$ is oriented towards $p\left(v_{i}\right)$, then orient all edges $v_{i} w$ towards $w$, for every $w \in C\left(v_{i}\right)$. Otherwise, let $C\left(v_{i}\right)=\left\{w_{1}, \ldots, w_{q}\right\}$. Note that $q \neq 1$, since $T$ has no vertex of degree 2 . If $\ell\left(v_{i}\right)$ is odd, then orient $w_{1} v_{i}$ and $w_{2} v_{i}$ towards $v_{i}$ and all the remaining edges $w v_{i}$ towards $w$, for $w \in\left\{w_{3}, \ldots, w_{q}\right\}$. If $\ell\left(v_{i}\right)$ is even, then orient $w_{1} v_{i}$ towards $v_{i}$ and all the remaining edges $w v_{i}$ towards $w$, for $w \in\left\{w_{2}, \ldots, w_{q}\right\}$.

In order to check that this orientation is proper, one may just observe that, by definition we cannot have two adjacent vertices with in-degree 0 , the only vertices with in-degree equal to 1 are leaves of $T$ and a vertex $v$ with in-degree 2 (resp. 3) is at even (resp. odd) distance from $r$. 


\section{Chapter 12}

\section{Hardness results}

In this chapter, we study the computational complexity of determining the proper orientation number of restricted bounded degree graphs and planar graphs.

Ahadi and Dehghan [3] showed that it is $\mathcal{N} \mathcal{P}$-complete to decide whether $\vec{\chi}(G) \leq 2$ for planar graphs $G$ by using a reduction from the PLANAR 3-SAT problem. We first improve this result by showing that it is $\mathcal{N} \mathcal{P}$-complete to decide whether the proper orientation number of planar subcubic graphs is at most 2 , where a subcubic graph is a graph with maximum degree 3 . We also prove the following more general result, losing, however, the planarity property: for every integer $k \geq 3$, it is $\mathcal{N} \mathcal{P}$-complete to determine whether $\vec{\chi}(G)<k$, for graphs $G$ with maximum degree $k$. These results are presented in Section 12.1. In Section 12.2 , we show that it is $\mathcal{N} \mathcal{P}$-complete to decide whether $\vec{\chi}(G) \leq 3$, for planar bipartite graphs $G$ with maximum degree 5 .

In the proofs of these results, we present reductions from variants of PlanaR 3-SAT. A 3-CNF formula is a boolean formula $\phi=(X, \mathcal{C})$ in conjunctive normal form in which $X$ is a set of $n$ variables, and $\mathcal{C}$ is a set of $m$ clauses such that every clause has exactly three literals and does not contain more than one literal of the same variable. The formula graph of a 3 -CNF formula $\phi=(X, \mathcal{C})$, denoted by $G(\phi)$, is a bipartite graph with vertex set $X \cup \mathcal{C}$ in which a variable-vertex $x$ is connected by an edge to a clause-vertex $C$ if and only if clause $C$ contains either the literal $x$ or $\neg x$. We say $\phi$ is planar if and only if the formula graph $G(\phi)$ is planar. The Planar 3-SAT problem is equivalent to the 3-SAT problem restricted to planar formulas. It is known that Planar 3-SAT is $\mathcal{N} \mathcal{P}$-complete [53].

For ease of notation, we denoted by $[t]$ the set of integers $\{1,2, \ldots, t\}$, for every $t \in \mathbb{N}$. Recall that an induced subgraph $H$ of a graph $G$ is $x$-pendant if $x \in V(H)$, and all edges between $V(H)$ and $V(G) \backslash V(H)$ are incident to $x$. In addition, if there exists a single edge $x y$ between $x$ and $y \in V(G) \backslash V(H)$, we say that $H$ is $x$-pendant at $y$. If $H$ is vertex-transitive, we simply say that $H$ is pendant at $y$.

\subsection{Planar subcubic graphs}

Recall that $\vec{\chi}(G) \leq \Delta(G)$, for any graph $G$. On the other hand, the following theorem shows that, for any integer $k \geq 3$, it is already $\mathcal{N} \mathcal{P}$-complete to determine whether $\vec{\chi}(G)<k$, for graphs $G$ with maximum degree $k$.

Theorem 12.1. Let $k$ be an integer such that $k \geq 3$. The following problem is $\mathcal{N} \mathcal{P}$-complete: INPUT: $A$ graph $G$ with $\Delta(G)=k$ and $\delta(G)=k-1$.

QUESTION : $\vec{\chi}(G) \leq k-1$ ? 
We first prove the case where $k=3$ and then show how to generalize the proof for larger values of $k$. Furthermore, the case $k=3$ has the additional characteristic, which is not true in the general case, that we may assume the graph $G$ to be planar.

In order to prove that it is $\mathcal{N} \mathcal{P}$-complete to decide whether the proper orientation number of a planar subcubic graph is at most 2, we modify the reduction proposed by Ahadi and Dehghan by using a different gadget for the variables of a 3-CNF formula. Let us first prove a lemma that allows us to force the orientation of some edges in any proper $k$-orientation of a graph.

Lemma 12.2. Let $k$ be a positive integer, and let $G$ be a graph containing a clique $K$ of size $k+1$. In any proper $k$-orientation of $G$, all edges between $V(K)$ and $V(G) \backslash V(K)$ are oriented from $V(K)$ to $V(G) \backslash V(K)$.

Proof. Consider a proper $k$-orientation $D$ of $G$. Clearly, all vertices of $K$ must have distinct in-degrees. Thus, for every $i \in\{0, \ldots, k\}$, there exists exactly one vertex in $K$ with in-degree $i$. It follows that $D[V(K)]$ is a transitive tournament, and every vertex in $K$ has all its in-neighbors belonging to $K$, where $D[V(K)]$ is the induced subdigraph of $D$ with vertices $V(K)$. Therefore, all edges in $G$ between $V(K)$ and $V(G) \backslash V(K)$ are oriented from $V(K)$ to $V(G) \backslash V(K)$.

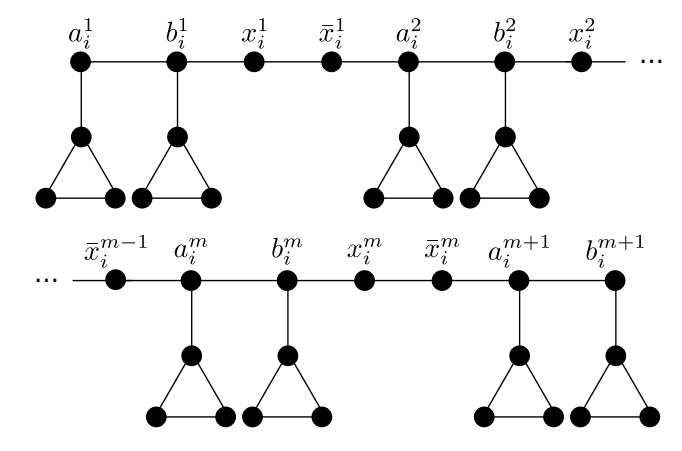

(a) $\mathrm{VG}_{i}$.

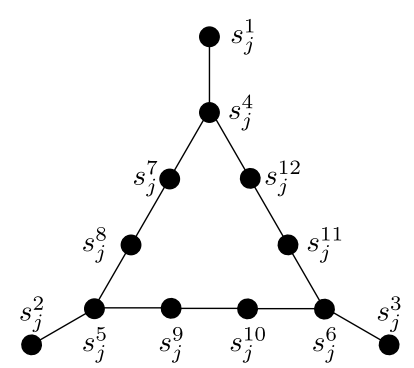

(b) $\mathrm{CG}_{j}$.

Figure 12.1: The variable gadget $\mathrm{VG}_{i}$ and the clause gadget $\mathrm{CG}_{j}$.

Theorem 12.3. The following problem is $\mathcal{N} \mathcal{P}$-complete:

INPUT : A planar graph $G$ with $\Delta(G)=3$ and $\delta(G)=2$.

QUESTION : $\vec{\chi}(G) \leq 2$ ?

Proof. This problem is trivially in $\mathcal{N P}$ because every proper 2-orientation is a short certificate.

Let $\phi=(X, \mathcal{C})$ be an instance of PlanAR 3-SAT where $X=\left\{x_{1}, \ldots, x_{n}\right\}$ is the set of variables and $\mathcal{C}=\left\{C_{1}, \ldots, C_{m}\right\}$ is the set of clauses. Additionally, let $G(\phi)$ be a formula graph of $\phi$. In what follows, we show how to construct a planar graph $G^{\prime}(\phi)$ such that $\vec{\chi}\left(G^{\prime}(\phi)\right) \leq 2$ if and only if $\phi$ is satisfiable.

Firstly, for every variable $x_{i}$ of $X$, we create a variable gadget $\mathrm{VG}_{i}$ (see Figure 12.1(a)). This gadget has $4 m+2$ vertices: $a_{i}^{j}, b_{i}^{j}$, for every $j \in[m+1]$, and $x_{i}^{j}, \bar{x}_{i}^{j}$ for every $j \in[m]$. These vertices form a path $P_{i}$, where $a_{i}^{j}$ is linked to $b_{i}^{j}$, for every $j \in[m+1] ; x_{i}^{j}$ is linked to $\bar{x}_{i}^{j}$ and to $b_{i}^{j}$, and $\bar{x}_{i}^{j}$ is linked to $a_{i}^{j+1}$, for every $j \in[m]$. In addition, for all $j \in[m+1]$, there is a pendant $K_{3}$ at $a_{i}^{j}$ and a pendant $K_{3}$ at $b_{i}^{j}$. For every clause $C_{j}$ in $\mathcal{C}$, we create a copy of the clause gadget $\mathrm{CG}_{j}$ depicted in Figure $12.1(\mathrm{~b})$.

To finish the construction of $G^{\prime}(\phi)$, we need to link the variable gadgets to the clause gadgets in such a way that the obtained graph is planar. Observe that we just need not to cross the edges linking the 
gadgets, because each gadget is itself planar and the formula graph $G(\phi)$ is planar. Consider a planar embedding $\tilde{G}(\phi)$ of $G(\phi)$. For each $x \in X$, let $\mathcal{C}_{x}=\{C \in \mathcal{C}: x \in C$ or $\neg x \in C\}$ and let $\pi_{x}:\left[\left|\mathcal{C}_{x}\right|\right] \rightarrow \mathcal{C}_{x}$ be a permutation of the elements in $\mathcal{C}_{x}$ in the clockwise order around $x$ in the planar embedding $\tilde{G}(\phi)$. In $G^{\prime}(\phi)$, for $t \in\left[\left|\mathcal{C}_{x}\right|\right]$ and $C_{j}=\pi_{x}(t)$, we link the vertex $x^{t}$ (resp. $\bar{x}^{t}$ ) in the gadget associated to the variable $x$ to one of the vertices $s_{j}^{1}, s_{j}^{2}$ or $s_{j}^{3}$. We link the vertices of the variable gadgets to the vertices of the clause gadgets in such a way that, for every $j \in[m]$, the vertices $s_{j}^{1}, s_{j}^{2}$ and $s_{j}^{3}$ have degree 2 in $G^{\prime}(\phi)$. Therefore, we have that $G^{\prime}(\phi)$ is planar, $\delta\left(G^{\prime}(\phi)\right)=2$ and $\Delta\left(G^{\prime}(\phi)\right)=3$.

Let us now prove that $\phi$ is satisfiable if and only if $\vec{\chi}\left(G^{\prime}(\phi)\right) \leq 2$.

Let $D$ be a proper 2-orientation of $G^{\prime}(\phi)$. Due to Lemma 12.2 and to the pendant $K_{3}$ at vertices of the path $P_{i}$, the in-degree of each vertex in $P_{i}$ must be either 1 or 2 , for every $i \in[n]$. Consequently, for every fixed $i \in[n]$, we either have $d_{D}^{-}\left(x_{i}^{j}\right)=1$ and $d_{D}^{-}\left(\bar{x}_{i}^{j}\right)=2$, or $d_{D}^{-}\left(x_{i}^{j}\right)=2$ and $d_{D}^{-}\left(\bar{x}_{i}^{j}\right)=1$, for all $j \in[m]$, that is, we have $d_{D}^{-}\left(x_{i}^{1}\right)=d_{D}^{-}\left(x_{i}^{2}\right)=\ldots=d_{D}^{-}\left(x_{i}^{m}\right)$ and $d_{D}^{-}\left(\bar{x}_{i}^{1}\right)=d_{D}^{-}\left(\bar{x}_{i}^{2}\right)=\ldots=d_{D}^{-}\left(\bar{x}_{i}^{m}\right)$, for all $i \in[n]$. Moreover, these in-degrees are just due to the orientations of the edges in the corresponding variable gadget. It means that every edge $x s \in E\left(G^{\prime}(\phi)\right)$ such that $x$ belongs to a variable gadget and $s$ belongs to a clause gadget is oriented from $x$ to $s$. Thus, for every $j \in[m]$ and $t \in\{1,2,3\}$, the vertex $s_{j}^{t}$ has in-degree at least 1 in $D$ and it must be different from the in-degree of its neighbor in a variable gadget.

Claim 12.4. In the clause gadget $\mathrm{CG}_{j}$, at least one of the vertices $s_{j}^{1}, s_{j}^{2}$ and $s_{j}^{3}$ has in-degree equal to 2.

Proof. Suppose to the contrary that $d_{D}^{-}\left(s_{j}^{1}\right)=d_{D}^{-}\left(s_{j}^{2}\right)=d_{D}^{-}\left(s_{j}^{3}\right)=1$. Thus, $s_{j}^{t}$ dominates $s_{j}^{t+3}$ and $d_{D}^{-}\left(s_{j}^{t+3}\right)=$ 2 , for all $t \in\{1,2,3\}$. Suppose first that $s_{j}^{12}$ dominates $s_{j}^{4}$. It follows that $s_{j}^{4}$ dominates $s_{j}^{7}$. Consequently, $s_{j}^{7}$ has in-degree 1 and dominates $s_{j}^{8}$, and so the edge $s_{j}^{5} s_{j}^{8}$ cannot be properly oriented (recall that $\left.d_{D}^{-}\left(s_{j}^{5}\right)=2\right)$. Similarly, one get a contradiction if $s_{j}^{4}$ dominates $s_{j}^{12}$. Therefore, we obtain that $s_{j}^{4}$ has in-degree 1 , a contradiction.

By the construction of $G^{\prime}(\phi)$, the orientation $D$ induces an assignment $\Gamma: X \rightarrow\{$ TRUE, FALSE $\}$ in which, for every $j \in[m], \Gamma(x)=$ TRUE if and only if $d_{D}^{-}\left(x^{j}\right)=1$. By Claim 12.4 , for each $j \in[m]$, there exists $t \in\{1,2,3\}$ such that $d_{D}^{-}\left(s_{j}^{t}\right)=2$. Thus, $\Gamma$ is a truth assignment that satisfies $\phi$.

Conversely, suppose that $\phi$ is satisfiable. Let $\Gamma: X \rightarrow\{$ TRUE, FALSE $\}$ be a truth assignment that satisfies $\phi$. Consider a variable $x \in X$. If $\Gamma(x)=$ TRUE, then orient $a^{j} b^{j}$ towards $b^{j}$ for every $j \in[m+1]$, and orient $b^{j} x^{j}$ towards $x^{j}, x^{j} \bar{x}^{j}$ towards $\bar{x}^{j}$ and $a^{j+1} \bar{x}^{j}$ towards $\bar{x}^{j}$ for every $j \in[m]$. If $\Gamma(x)=$ FALSE, then orient $b^{j} a^{j}$ towards $a^{j}$ for every $j \in[m+1]$, and orient $b^{j} x^{j}$ towards $x^{j}, \bar{x}^{j} x^{j}$ towards $x^{j}$ and $a^{j+1} \bar{x}^{j}$ towards $\bar{x}^{j}$ for every $j \in[m]$. Thus, for every $j \in[m]$, if $\Gamma(x)=$ TRUE, then $d^{-}\left(x^{j}\right)=1$ and $d^{-}\left(\bar{x}^{j}\right)=2$, otherwise, $d^{-}\left(x^{j}\right)=2$ and $d^{-}\left(\bar{x}^{j}\right)=1$. Since $\Gamma$ satisfies $\phi$, no clause has three false literals. Consequently, no clause gadget has all three vertices $s^{1}, s^{2}$ and $s^{3}$ forced to have in-degree 1 due to neighbors of in-degree 2 in the corresponding variable gadgets. In this case, we can extend this proper 2-orientation of the variables gadgets to a proper 2-orientation of $G^{\prime}(\phi)$ using one of the suitable clause orientations depicted in Figure 12.2. Therefore, we have $\vec{\chi}\left(G^{\prime}(\phi)\right) \leq 2$.

The reduction presented in the proof of Theorem 12.3 can be easily adapted to prove Theorem 12.1.

Proof of Theorem 12.1. Let $\phi$ be a 3-CNF formula and let $G^{\prime}(\phi)$ be the graph described in the proof of Theorem 12.3. Let $k \geq 3$ be an integer and let $G^{\prime \prime}(\phi)$ be the graph obtained from $G^{\prime}(\phi)$ by adding $k-3$ pendant $K_{k}$ at each vertex of $G^{\prime}(\phi)$, and replacing each pendant $K_{3}$ at a vertex by a pendant $K_{k}$ at the same vertex. By the construction, one can easily check that $\delta\left(G^{\prime \prime}(\phi)\right)=k-1$ and $\Delta\left(G^{\prime \prime}(\phi)\right)=k$. 


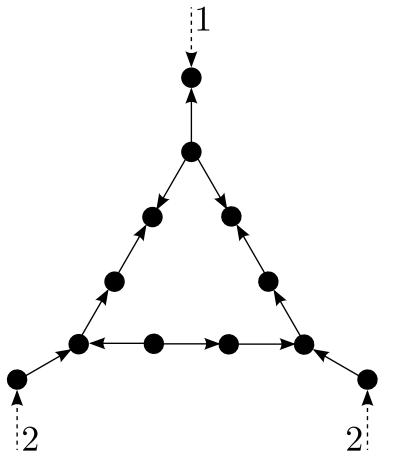

(a)

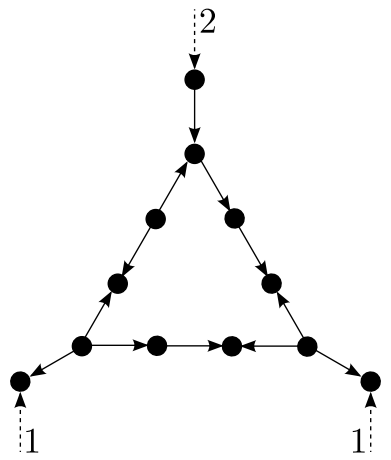

(b)

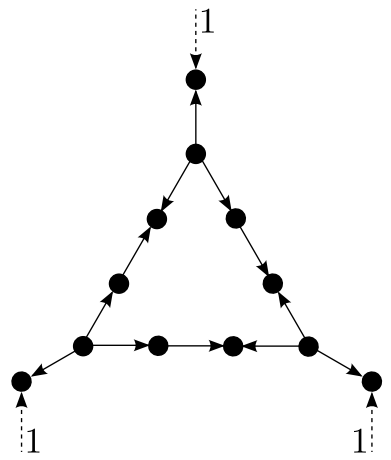

(c)

Figure 12.2: Suitable clause orientations.

Note that, in every proper $(k-1)$-orientation, all the vertices of $G^{\prime \prime}(\phi)$ except those belonging to the pendant $K_{k}$ 's have in-degree equal to $k-1$ or $k-2$. Therefore, using similar arguments to those presented in the proof of Theorem 12.3, we conclude that $\phi$ is satisfiable if, and only if, $\vec{\chi}\left(G^{\prime \prime}(\phi)\right) \leq k-1$.

Theorem 12.5. The following problem is $\mathcal{N} \mathcal{P}$-complete:

INPUT : A planar graph $G$ with $\Delta(G)=4$ and $\delta(G)=3$.

QUESTION : $\vec{\chi}(G) \leq 3$ ?

Proof. Considering a planar 3-CNF formula and observing that the complete graph on four vertices $K_{4}$ is planar, the result follows directly from the proof of Theorem 12.1.

\subsection{Planar bipartite graphs}

In this section, we prove that computing the proper orientation number of a graph is still $\mathcal{N} \mathcal{P}$-hard for planar bipartite graphs of bounded degree. The idea of our reduction is roughly the same as in Theorems 12.1 and 12.3, although we use another class of 3-SAT formulas in order to obtain bipartite instances and the gadgets of the reduction are more complicated.

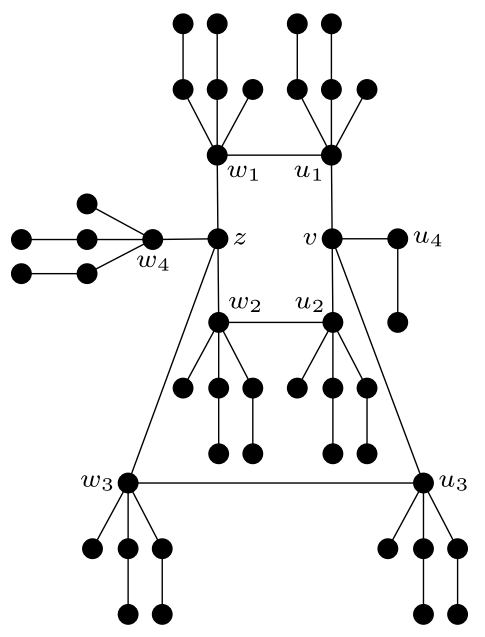

Figure 12.3: The bipartite graph $B_{5}$. 
The gadget we use to replace the pendant cliques of the proofs of Theorems 12.1 and 12.3 is the graph $B_{5}$, depicted in Figure 12.3. The following lemma, analogously to Lemma 12.2, allows us to force the orientation of some edges in any proper 3-orientation of a graph.

Lemma 12.6. Let $G$ be a graph with a v-pendant $B_{5}$ at $y$. In any proper 3-orientation of $G, y$ is dominated by $v$.

Proof. Suppose to the contrary that there exists a proper 3-orientation $D$ of $G$ such that $y$ dominates $v$.

If $d^{-}(v)=1$, then $v$ dominates $u_{i}$ for all $i \in\{1,2,3,4\}$. Moreover, by Lemma 11.10, $d^{-}\left(u_{i}\right)=3$ for $i \in\{1,2,3\}$. Thus, for $i \in\{1,2,3\}, d^{-}\left(w_{i}\right) \leq 2$. Hence, again by Lemma 11.10, $w_{i}$ dominates $u_{i}$ and $z$. It follows that $d^{-}(z)=3$ and $z$ dominates $w_{4}$. This contradicts Lemma 11.10.

If $d^{-}(v)=2$, then $v$ is dominated by $u_{4}$, otherwise $u_{4}$ has the same in-degree as one of its two neighbors. Thus, $v$ dominates $u_{i}$ for $i \in\{1,2,3\}$. Similarly to the previous case, we obtain a contradiction.

If $d^{-}(v)=3$, then $v$ dominates $u_{i}$, for some $i \in\{1,2,3\}$. Therefore, $d^{-}\left(u_{i}\right) \in\{1,2\}$, which contradicts Lemma 11.10 .

By Theorem 11.1, all planar bipartite graphs with maximum degree 5 have proper orientation at most 4 . On the other hand, we next show that it is $\mathcal{N} \mathcal{P}$-complete to decide whether $\vec{\chi}(G) \leq 3$ for such graphs.

Theorem 12.7. The following problem is $\mathcal{N} \mathcal{P}$-complete:

INPUT : A planar bipartite graph $G$ with $\Delta(G)=5$.

QUESTION : $\vec{\chi}(G) \leq 3$ ?

Proof. Again, this problem is in $\mathcal{N} \mathcal{P}$ because any proper 3-orientation of $G$ is a certificate.

A 3-SAT formula $\phi$ is said to be monotone if each clause has only positive literals (a positive clause) or only negative literals (a negative clause). Recall that a 3 -CNF formula $\phi$ is planar if the formula graph $G(\phi)$ is planar. The problem of deciding whether planar monotone 3-SAT formulas are satisfiable was shown to be $\mathcal{N} \mathcal{P}$-complete [11]. Let $\phi$ be a planar monotone 3-CNF formula with clauses $\mathcal{C}=\left\{C_{1}, \ldots, C_{m}\right\}$ and variables $X=\left\{x_{1}, \ldots, x_{n}\right\}$ and let $G(\phi)$ be the formula graph corresponding to $\phi$. In what follows, we show how to construct a planar bipartite graph $G^{\prime}(\phi)$ with $\Delta(G)=5$ such that $\vec{\chi}\left(G^{\prime}(\phi)\right) \leq 3$ if and only if $\phi$ is satisfiable.

Firstly, for each variable $x_{i}$, we create a variable gadget $\mathrm{VG}_{i}^{\prime}$ depicted in Figure 12.4(a). This gadget has $4 m+2$ vertices: $a_{i}^{j}, b_{i}^{j}$ for every $j \in[m+1]$, and $x_{i}^{j}, \bar{x}_{i}^{j}$ for every $j \in[m]$. These vertices form a path $P_{i}$, where $a_{i}^{j}$ is linked to $b_{i}^{j}$, for every $j \in[m+1] ; x_{i}^{j}$ is linked to $\bar{x}_{i}^{j}$ and to $b_{i}^{j}$, and $\bar{x}_{i}^{j}$ is linked to $a_{i}^{j+1}$, for every $j \in[m]$. The gadget $\mathrm{VG}_{i}^{\prime}$ also contains $6 m+4 v$-pendant $B_{5}$ : two at $a_{i}^{j}$, two at $b_{i}^{j}$, for every $j \in[m+1]$, one at $x_{i}^{j}$ and another at $\bar{x}_{i}^{j}$ for every $j \in[m]$ (these $B_{5}$ graphs are represented by the dashed white circles in Figure 12.4(a)).

For every clause $C_{j}$ of $C$, we create a clause gadget $\mathrm{CG}_{j}^{\prime}$ as depicted in Figure 12.4(b). It is obtained from two paths $s_{j}^{1} s_{j}^{4} \ldots s_{j}^{10} s_{j}^{3}$ and $s_{j}^{11} s_{j}^{12} s_{j}^{13} s_{j}^{2}$ linked by the edge $s_{j}^{7} s_{j}^{11}$. Moreover, there exists a $v$-pendant $B_{5}$ at all these vertices, except at $s_{j}^{13}$, and we have an additional $v$-pendant $B_{5}$ at $s_{j}^{12}$ (these $B_{5}$ graphs are represented by the dashed white circles in Figure 12.4(b)).

To finish the construction of $G^{\prime}(\phi)$, we need to link the variable gadgets to the clause gadgets in such a way that the obtained graph is planar. Observe that we just need not to cross the edges linking the gadgets since each gadget is itself planar and the formula graph $G(\phi)$ is planar. We can accomplish this in the same way as described in the proof of Theorem 12.3. Since each clause has exactly three literals, we add these edges so that, for every $j \in[m]$, the vertices $s_{j}^{1}, s_{j}^{2}$ and $s_{j}^{3}$ have degree 3 in $G^{\prime}(\phi)$. Thus, $G^{\prime}(\phi)$ is planar, and $\Delta\left(G^{\prime}(\phi)\right)=5$ due to the graph $B_{5}$. 


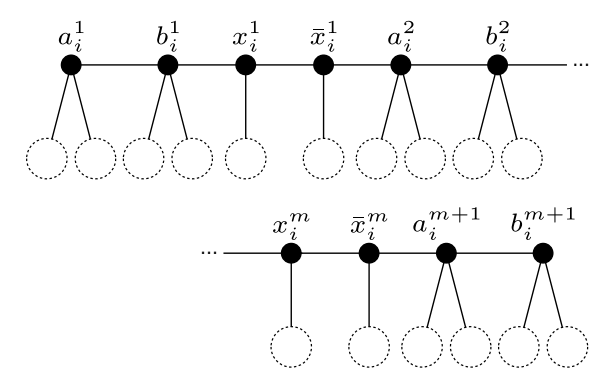

(a) Variable gadget $\mathrm{VG}_{i}^{\prime}$.

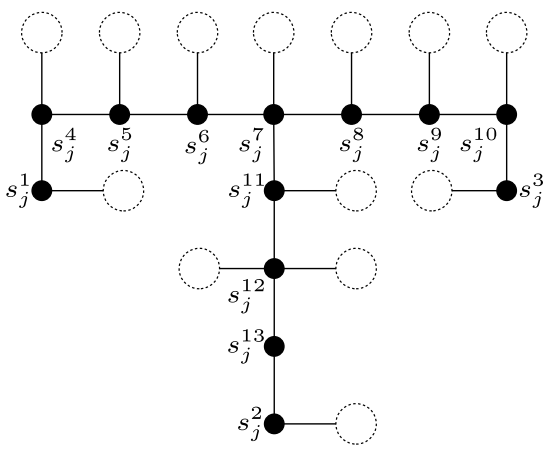

(b) Clause gadget $\mathrm{CG}_{j}^{\prime}$.

Figure 12.4: Every dashed circle represents a copy of $B_{5}$.

Using the monotonicity of formula $\phi$, we next prove that $G^{\prime}(\phi)$ is bipartite. Clearly, we can properly color the vertices of each variable gadget with colors $A$ and $B$. Suppose that we assign the color $A$ to the vertex $x_{i}^{j}$, for each $i \in[n]$ and $j \in[m]$. For each $j \in[m]$, we can assign the same color $(A$ or $B)$ to the vertices $s_{j}^{1}, s_{j}^{2}$ and $s_{j}^{3}$ since $\phi$ is monotone. Note that we can easily extend this partial coloring to the other vertices in each clause and variable gadget in $G^{\prime}(\phi)$. Thus, $G^{\prime}(\phi)$ is bipartite.

Let us now prove that $\vec{\chi}\left(G^{\prime}(\phi)\right) \leq 3$ if and only if $\phi$ is satisfiable.

Suppose first that $\vec{\chi}\left(G^{\prime}(\phi)\right) \leq 3$. Let $D$ be a proper 3-orientation of $G^{\prime}(\phi)$. By Lemma 12.6 and due to the $v$-pendant $B_{5}$ graphs that are attached to the vertices in each path $P_{i}$, the in-degrees of the vertices in this path are either 2 or 3 , for every $i \in[n]$. Consequently, for a given $i \in[n]$, we either have $d_{D}^{-}\left(x_{i}^{j}\right)=2$ and $d_{D}^{-}\left(\bar{x}_{i}^{j}\right)=3$, or $d_{D}^{-}\left(x_{i}^{j}\right)=3$ and $d_{D}^{-}\left(\bar{x}_{i}^{j}\right)=2$, for all $j \in[m]$. Moreover, these in-degrees are just due to the orientations of the edges belonging to the corresponding variable gadget. Thus, every edge $x s \in E\left(G^{\prime}(\phi)\right)$ such that $x$ belongs to a variable gadget and $s$ belongs to a clause gadget is oriented from $x$ to $s$. Consequently, for each $j \in[m]$ and $t \in\{1,2,3\}$, the vertex $s_{j}^{t}$ has in-degree at least 2 in $D$ since it has a $v$-pendant $B_{5}$ attached to it.

Claim 12.8. In every proper 3 -orientation of $G^{\prime}(\phi)$, for every $j \in[m]$, one of the vertices $s_{j}^{1}, s_{j}^{2}$ and $s_{j}^{3}$ has in-degree 3.

Proof. Suppose to the contrary that there exists a proper 3-orientation $D$ of $G^{\prime}(\phi)$ such that $d_{D}^{-}\left(s_{j}^{1}\right)=$ $d_{D}^{-}\left(s_{j}^{2}\right)=d_{D}^{-}\left(s_{j}^{3}\right)=2$, for some $j \in[m]$. Thus, the edges $s_{j}^{2} s_{j}^{13}, s_{j}^{13} s_{j}^{12}, s_{j}^{12} s_{j}^{11}$ and $s_{j}^{11} s_{j}^{7}$ are oriented from $s_{j}^{2}$ to $s_{j}^{13}, s_{j}^{13}$ to $s_{j}^{12}, s_{j}^{12}$ to $s_{j}^{11}$, and from $s_{j}^{11}$ to $s_{j}^{7}$ in $D$. Since $s_{j}^{7}$ is adjacent to a vertex with in-degree 2 and it has in-degree at least 2 in this orientation, it is dominated by either $s_{j}^{6}$ or $s_{j}^{8}$. If $s_{j}^{6}$ dominates $s_{j}^{7}$, then the edges $s_{j}^{7} s_{j}^{8}, s_{j}^{8} s_{j}^{9}$, and $s_{j}^{9} s_{j}^{10}$ are oriented from $s_{j}^{7}$ to $s_{j}^{8}, s_{j}^{8}$ to $s_{j}^{9}$, and from $s_{j}^{10}$ to $s_{j}^{9}$. Therefore, we have $d_{D^{\prime}}^{-}\left(s_{j}^{3}\right)=d_{D^{\prime}}^{-}\left(s_{j}^{10}\right)=2$, a contradiction. By symmetry, we obtain a similar contradiction if $s_{j}^{8}$ dominates $s_{j}^{7}$.

Let $\Gamma: X \rightarrow\{$ TRUE, FALSE $\}$ be the truth assignment defined as $\Gamma(x)=$ TRUE if and only if $d_{D}^{-}\left(x^{j}\right)=2$ for some $j \in[m]$. By Claim 12.8, $\Gamma$ satisfies $\phi$.

Reciprocally, suppose that $\phi$ is satisfiable. Let $\Gamma: X \rightarrow\{$ TRUE, FALSE $\}$ be a truth assignment that satisfies $\phi$.

First, orient each $v$-pendant $B_{5}$ belonging to a variable gadget according to Figure 12.5(a). Moreover, for each $v$-pendant $B_{5}$ in $G^{\prime}(\phi)$, orient the edge between $v$ and $y$, the only neighbor of $v$ in $V\left(G^{\prime}(\phi)\right) \backslash$ $V\left(B_{5}\right)$, towards $y$. Note that the in-degree of $v$ is equal to 1 in every $v$-pendant $B_{5}$ belonging to a variable 


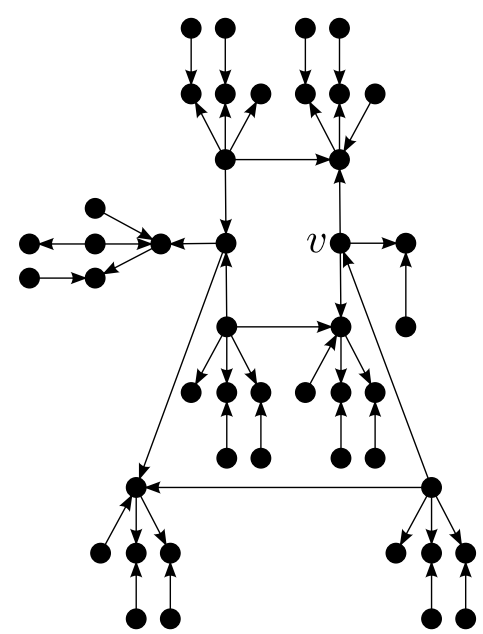

(a) Vertex $v$ has in-degree 1 .

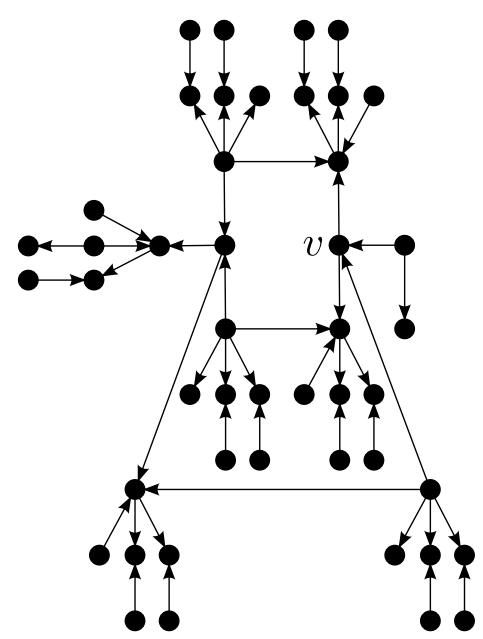

(b) Vertex $v$ has in-degree 2 .

Figure 12.5: Two proper 3-orientations of $B_{5}$.

gadget. Consider a variable $x \in X$. We define an orientation of $G^{\prime}(\phi)$ as follows:

- If $\Gamma(x)=$ TRUE, orient $a^{j} b^{j}$ towards $b^{j}$ for every $j \in[m+1]$, and orient $b^{j} x^{j}$ towards $x^{j}, x^{j} \bar{x}^{j}$ towards $\bar{x}^{j}$ and $a^{j+1} \bar{x}^{j}$ towards $\bar{x}^{j}$ for every $j \in[m]$ (see Figure 12.6(a)).

- If $\Gamma(x)=$ FALSE, orient $b^{j} a^{j}$ towards $a^{j}$ for every $j \in[m+1]$, and orient $b^{j} x^{j}$ towards $x^{j}, \bar{x}^{j} x^{j}$ towards $x^{j}$ and $a^{j+1} \bar{x}^{j}$ towards $\bar{x}^{j}$ for every $j \in[m]$ (see Figure 12.6(b)).

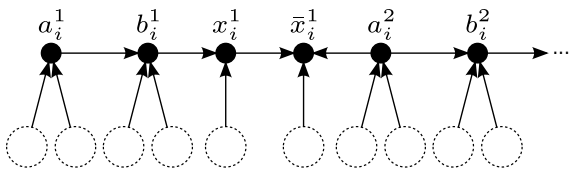

(a) Orientation True.

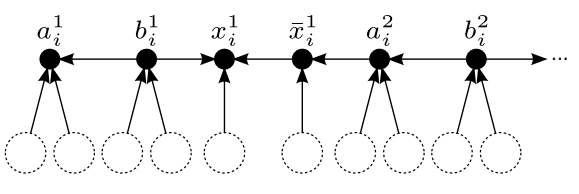

(b) Orientation FALSE.

Figure 12.6: Suitable orientations of the gadget $x_{i}$.

Thus, if $\Gamma(x)=$ TRUE, then $d_{D}^{-}\left(x^{j}\right)=2$ and $d_{D}^{-}\left(\bar{x}^{j}\right)=3$, otherwise $d_{D}^{-}\left(x^{j}\right)=3$ and $d_{D}^{-}\left(\bar{x}^{j}\right)=2$, for every $j \in[m]$. Since $\Gamma$ satisfies $\phi$, no clause has three false literals. Consequently, in every clause gadget, at least one of the vertices $s^{1}, s^{2}$ and $s^{3}$ has in-degree different from 2 due to its neighbors in the corresponding variable gadgets. Thus, we can extend this proper 3-orientation of the variables gadgets to a proper 3-orientation of $G^{\prime}(\phi)$ as follows:

- For every $w s \in E\left(G^{\prime}(\phi)\right)$ such that $w$ belongs to a variable gadget and $s$ belongs to a clause gadget, orient this edge from $w$ to $s$.

- Then, use one of the suitable clause orientations depicted in Figure 12.7.

- Finally, we orient each $B_{5}$ belonging to a clause gadget. Once more, for each $v$-pendant $B_{5}$ in $G^{\prime}(\phi)$, orient the edge between $v$ and $y$, the only neighbour of $v$ in $V\left(G^{\prime}(\phi)\right) \backslash V\left(B_{5}\right)$, towards $y$. Observe that, at this moment, all edges incident to $y$ have already been oriented. If the in-degree of $y$ equals 2, then orient the corresponding $B_{5}$ as depicted in Figure 12.5(a). Otherwise, we orient the corresponding $B_{5}$ as depicted in Figure 12.5(b). 
Note that the orientation of $G^{\prime}(\phi)$ described above is proper and each vertex has in-degree at most 3 . Therefore, $\vec{\chi}\left(G^{\prime}(\phi)\right) \leq 3$.

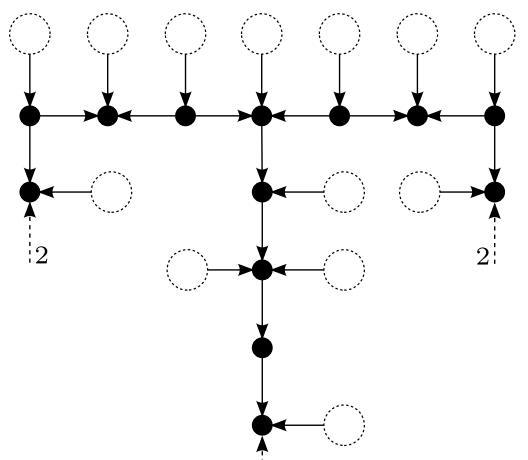

2

(a)

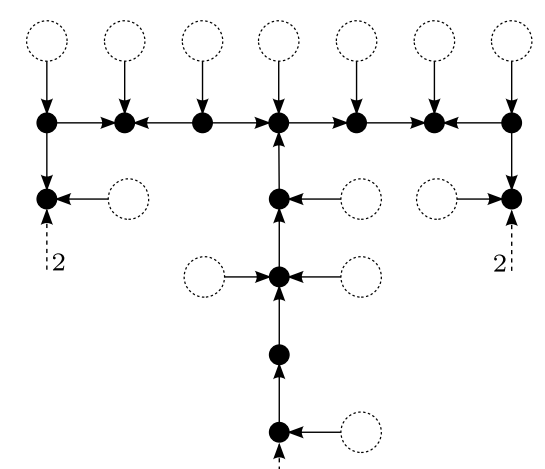

3

(c)

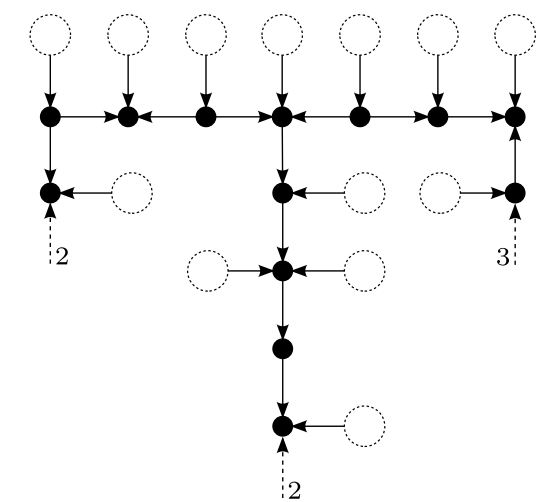

(b)

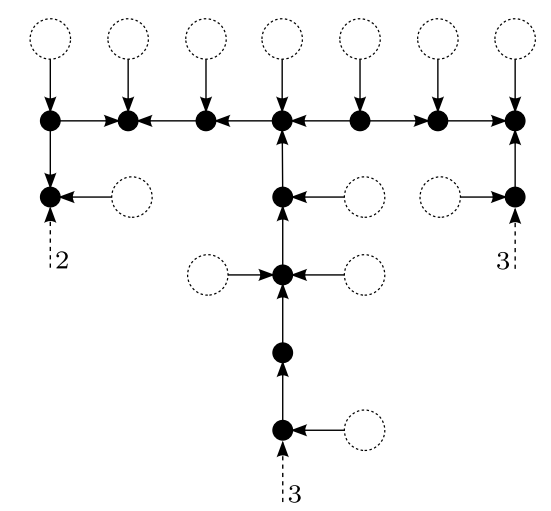

(d)

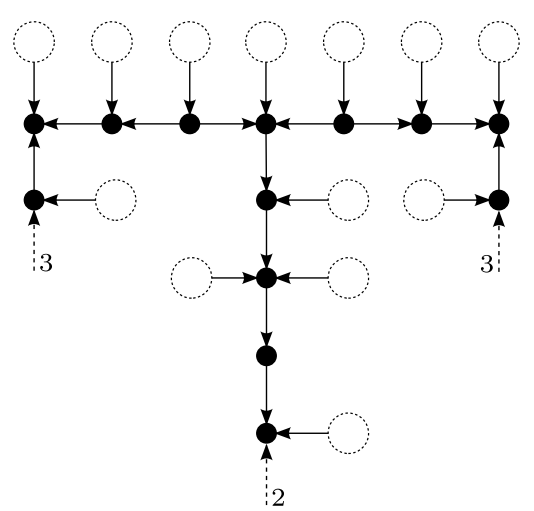

(e)

Figure 12.7: Suitable 3-orientations of the clause gadgets. 


\section{Part V}

\section{Digraph subdivision}





\section{Chapter 13}

\section{Finding subdivisions of digraphs}

\subsection{Basic definitions and known results}

A subdivision of a graph $G$ is a graph obtained from $G$ by replacing some of its edges by internally vertex-disjoint paths, that is, in a subdivision of $G$, for each $u v \in E(G)$, edge $u v$ is replaced by a path $P_{u v}$ with endpoints $u$ and $v$, and newly created internal vertices. In the world of digraphs, arcs are replaced by directed paths. More precisely, a subdivision of a digraph $D$ is a digraph obtained from $D$ by replacing some of its arcs by internally vertex-disjoint directed paths with the same endpoints and oriented in the same direction as the corresponding arcs. An example of subdivision of a digraph is illustrated in Figure 13.1.

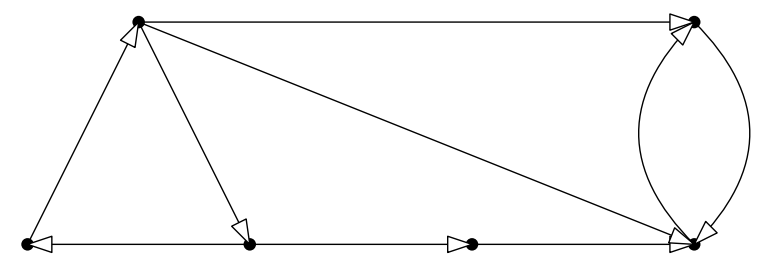

(a) A digraph $D$.

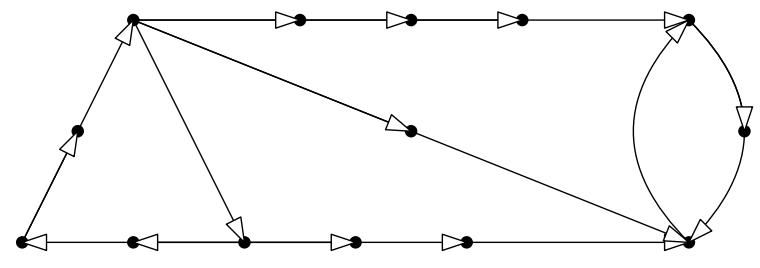

(b) A subdivision of $D$.

Figure 13.1: Example of subdivision of a digraph.

Given two (di)graphs $H$ and $G$, we say that $G$ contains a subdivision of $H$ if there exists a subgraph (respectively, subdigraph) $G^{\prime}$ of $G$ such that $G^{\prime}$ is isomorphic to some subdivision of $H$.

Let $k \in \mathbb{N}$. We denote by $K_{k}$ the complete (undirected) graph on $k$ vertices. The complete digraph on $k$ vertices, denoted by $\vec{K}_{k}$, is obtained from $K_{k}$ replacing every edge of it by two arcs with the same endpoints and opposite directions, that is, every edge of $K_{k}$ is replaced by a copy of $\vec{C}_{2}$, the directed cycle on 2 vertices. A tournament on $k$ vertices is an orientation of the complete graph $K_{k}$. The transitive tournament on $k$ vertices, denoted by $T T_{k}$, is a tournament on $k$ vertices with no directed cycle. The $K_{4}$, $\vec{K}_{4}$ and $T T_{4}$ are depicted in Figure 13.2.

In the sixties, Mader [55] established the following theorem for (undirected) graphs.

Theorem 13.1 (Mader [55]). For every positive integer $k$, there exists an integer $f(k)$ such that every graph with minimum degree at least $f(k)$ contains a subdivision of $K_{k}$, the complete graph on $k$ vertices.

For $k \leq 4$, we have $f(k)=k-1$ as first proved by Dirac [35]; for $k=5$, we have the estimate 


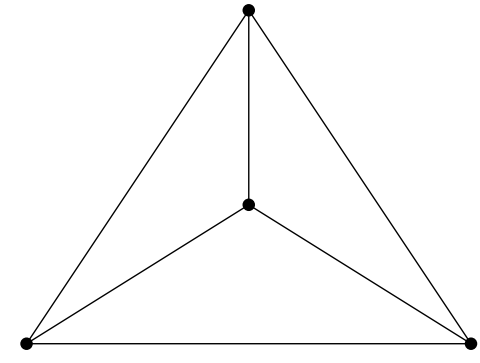

(a) $K_{4}$, the complete graph on 4 vertices.

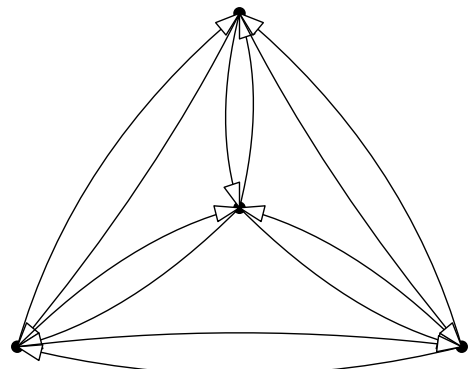

(b) $\vec{K}_{4}$, the complete digraph on 4 vertices.

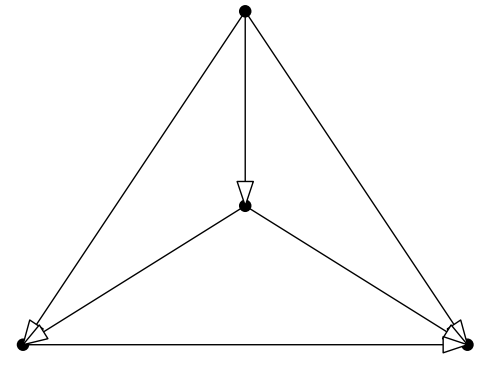

(c) $T T_{4}$, the transitive tournament on 4 vertices.

Figure 13.2: Example of a complete digraph and a transitive tournament.

$6 \leq f(5) \leq 7$ by Thomassen [74, 75]. In general, the order of growth of $f(k)$ is $k^{2}$ as shown in [13] and [52].

Similarly, it would be interesting to find analogous results for digraphs. However, the obvious analogue that a digraph with sufficiently large minimum in-degree and minimum out-degree contains a subdivision of the complete digraph of order $n$ is false as shown by Mader [56]

Let $\gamma$ be a digraph parameter. A digraph $F$ is $\gamma$-maderian if there exists a least integer $\operatorname{mader}_{\gamma}(F)$ such that every digraph $D$ with $\gamma(D) \geq \operatorname{mader}_{\gamma}(F)$ contains a subdivision of $F$.

For a digraph $D, \delta^{+}(D)$ (respectively, $\delta^{-}(D)$ ) denotes the minimum out-degree (respectively, indegree) and $\delta^{0}(D)=\min \left\{\delta^{+}(D), \delta^{-}(D)\right\}$. A natural question is to ask what digraphs $F$ are $\delta^{+}$-maderian (respectively, $\delta^{0}$-maderian). Observe that every $\delta^{+}$-maderian digraph is also $\delta^{0}$-maderian and that mader $\delta^{+} \geq$ $\operatorname{mader}_{\delta^{0}}$.

On the positive side, Mader conjectured that every acyclic digraph is $\delta^{+}$-maderian. Since every acyclic digraph is the subdigraph of the transitive tournament on the same order, it is enough to prove that transitive tournaments are $\delta^{+}$-maderian.

Conjecture 13.2 (Mader [56]). For every positive integer $k$, there exists a least integer mader $_{\delta^{+}}\left(T T_{k}\right)$ such that every digraph $D$ with $\delta^{+}(D) \geq \operatorname{mader}_{\delta^{+}}\left(T T_{k}\right)$ contains a subdivision of $T T_{k}$.

Mader proved that $\operatorname{mader}_{\delta^{+}}\left(T T_{4}\right)=3$, but even the existence of mader $_{\delta^{+}}\left(T T_{5}\right)$ is still open.

This conjecture implies directly that transitive tournaments (and thus all acyclic digraphs) are $\delta^{0}$ maderian.

Conjecture 13.3. For every positive integer $k$, there exists a least integer $\operatorname{mader}_{\delta^{0}}\left(T T_{k}\right)$ such that every digraph $D$ with $\delta^{0}(D) \geq \operatorname{mader}_{\delta^{0}}\left(T T_{k}\right)$ contains a subdivision of $T T_{k}$.

In fact, Conjecture 13.3 is equivalent to Conjecture 13.2 because if transitive tournaments are $\delta^{0}$ maderian, then mader $\delta^{+}\left(T T_{k}\right) \leq \operatorname{mader}_{\delta^{0}}\left(T T_{2 k}\right)$ for all $k$. Let $D$ be a digraph with minimum out-degree mader $_{\delta^{0}}\left(T T_{2 k}\right)$, and let $D^{\prime}$ be the digraph obtained from the disjoint union of a copy of $D$ and a copy of its converse (the digraph obtained from $D$ by reversing all arcs) $\bar{D}$ by adding all $\operatorname{arcs}$ from $\bar{D}$ to $D$. Clearly, $\delta^{0}\left(D^{\prime}\right) \geq \operatorname{mader}_{\delta^{0}}\left(T T_{2 k}\right)$. Therefore $D^{\prime}$ contains a subdivision of $T T_{2 k}$. Hence, either $D$ or $\bar{D}$ (and so $D$ ) contains a subdivision of $T T_{k}$.

Although both conjectures are equivalent, the above reasoning does not prove that every $\delta^{0}$-maderian digraph is also $\delta^{+}$-maderian. The case of oriented trees (that is, orientations of undirected trees) is typical. Using a simple greedy procedure, one can easily find every oriented tree of order $k$ in every digraph with 


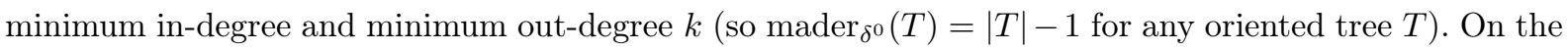
other hand, it is still open whether oriented trees are $\delta^{+}$-maderian and a natural important step towards Conjecture 13.2 would be to prove the following weaker one.

Conjecture 13.4. Every oriented tree is $\delta^{+}$-maderian.

Conjecture 13.2 implies that the acyclic digraphs are $\delta^{+}$-maderian. However, they are not the only ones. For example, it is folklore that every digraph with minimum out-degree at least 1 contains a directed cycle, which is a subdivision of $\vec{C}_{2}$, the directed 2-cycle. More generally, one can easily show, by considering a directed path of maximum length, that every digraph with minimum out-degree at least $k-1$ contains a directed cycle of length at least $k$. In other words, $\operatorname{mader}_{\delta^{+}}\left(\vec{C}_{k}\right)=k-1$, where $\vec{C}_{k}$ denotes the directed $k$-cycle. Furthermore Alon [5] showed that every digraph with minimum out-degree at least $64 k$ contains $k$ disjoint directed cycles, which forms a subdivision of the disjoint union of $k$ copies of $\vec{C}_{2}$. A celebrated conjecture of Bermond and Thomassen [12] states that the bound $64 k$ can be decreased to $2 k-1$.

Conjecture 13.5 (Bermond and Thomassen [12]). For every positive integer $k$, every digraph with minimum out-degree at least $2 k-1$ contains $k$ disjoint directed cycles.

In [5], Alon also conjectured the following.

Conjecture 13.6 (Alon [5]). There exists a function $h$ such that every digraph with minimum out-degree $h(k)$ has a partition $\left(V_{1}, V_{2}\right)$ such that, for $i \in\{1,2\}, D\left[V_{i}\right]$ has minimum out-degree $k$.

The difficulty of this question is remarkable, as the existence of $h(2)$ still remains open. If true, this conjecture implies the following one.

Conjecture 13.7. If $H_{1}$ and $H_{2}$ are $\delta^{+}$-maderian, then the disjoint union of $H_{1}$ and $H_{2}$ is also $\delta^{+}$maderian.

Partial positive answers to this conjecture can be obtained via the Erdős-Pósa Property. Let $H$ denote a digraph. For every positive integer $k$, we denote by $k \times H$ the disjoint union of $k$ copies of $H$. Digraph $H$ is said to have the Erdôs-Pósa Property if there exists a function $\phi: \mathbb{N} \rightarrow \mathbb{N}$ such that, for each $k \in \mathbb{N}$, every digraph $D$ either contains a subdivision of $k \times H$, or has a set of vertices $S$ of size at most $\phi(k)$ such that $D-S$ contains no subdivision of $H$.

Theorem 13.8. If $H$ is $\delta^{+}$-maderian and has the Erdôs-Pósa Property, then $k \times H$ is also $\delta^{+}$-maderian for all positive integer $k$.

Proof. Let $H$ be a maderian digraph having the Erdős-Pósa Property for some function $\phi$. Let $D$ be a digraph with $\delta^{+}(D) \geq \phi(k)+\operatorname{mader}_{\delta^{+}}(H)$. For every set $S \subseteq V(D)$ of size at most $\phi(k), \delta^{+}(D-S) \geq$ $\operatorname{mader}_{\delta^{+}}(H)$, so $D-S$ contains a subdivision of $H$. Thus, since $H$ has the Erdôs-Pósa Property, $D$ contains a subdivision of $k \times H$.

Reed et al. [68] proved that $\vec{C}_{2}$ has the Erdós-Pósa Property, and using the Directed Grid Theorem [49], Akhoondian et al. [4] showed that many digraphs have the Erdôs-Pósa Property, in particular all disjoint unions of directed cycles. Hence, Theorem 13.8 implies that disjoint unions of directed cycles are $\delta^{+}$maderian. 


\subsection{Contributions}

We give evidences to Conjecture 13.4 in Chapter 14. First, in Section 14.1, we prove that every oriented path (that is, orientation of an undirected path) $P$ is $\delta^{+}$-maderian and that mader $_{\delta^{+}}(P)=|V(P)|-1$. In Section 14.2, we consider arborescences. An out-arborescence (respectively, in-arborescence) is an oriented tree in which all arcs are directed away from (respectively, towards) a vertex called root. Trivially, the simple greedy procedure shows that $\operatorname{mader}_{\delta^{+}}(T)=|T|-1$ for every out-arborescence. In contrast, the fact that in-arborecences are $\delta^{+}$-maderian is not obvious since we have no control on the in-degree of every vertex in a digraph of large minimum out-degree. We shall prove that the in-arborescences are $\delta^{+}$-maderian.

The mentioned contributions were obtained in collaboration with Stéphan Thomassé during a research visit at École Normale Supérieure de Lyon in France from September 2015 to August 2016. Additionally, these results are presented in a joint paper with Pierre Aboulker, Nathann Cohen, Frédéric Havet and William Lochet (see manuscript [1]). 


\section{Chapter 14}

\section{Digraphs with large out-degree}

\subsection{Subdivisions of oriented paths}

Let $P=x_{1} x_{2} \cdots x_{n}$ be an oriented path with $n$ vertices. We say that $P$ is an $\left(x_{1}, x_{n}\right)$-path. The initial vertex of $P$ is $x_{1}$ and the terminal vertex of $P$ is $x_{n} . P$ is a directed path (or simply, a dipath) if $x_{i}$ dominates $x_{i+1}$ for all $i \in[n-1]$.

Let $k_{1}$ be a nonnegative integer and $k_{2}, \ldots, k_{\ell}$ be positive integers. We denote by $P\left(k_{1}, k_{2}, \ldots, k_{\ell}\right)$ the oriented path obtained from an undirected path $v_{1} v_{2} \ldots v_{\ell+1}$ by replacing, for every $i \in[\ell]$, the edge $\left\{v_{i}, v_{i+1}\right\}$ by a directed path of length $k_{i}$ from $v_{i}$ to $v_{i+1}$ if $i$ is odd, and from $v_{i+1}$ to $v_{i}$ if $i$ is even. (If $k_{1}=0$, then $v_{1}=v_{2}$.) In Figure 14.1 is depicted the oriented path $P(2,3,1)$ obtained from a path $P$ on 4 vertices.

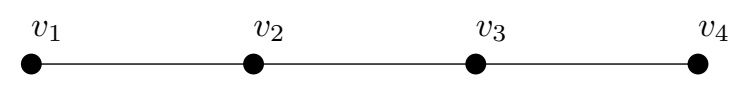

(a) Path $P=v_{1} v_{2} v_{3} v_{4}$.

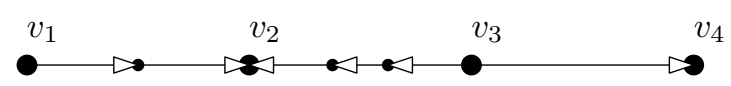

(b) Oriented path $P(2,3,1)$.

Figure 14.1: Example of oriented path.

Theorem 14.1. Let $P\left(k_{1}, k_{2}, \ldots, k_{\ell}\right)$ be an oriented path, and let $D$ be a digraph with $\delta^{+}(D) \geq \sum_{i=1}^{\ell} k_{i}$. For every $v \in V(D), D$ contains a path $P\left(k_{1}^{\prime}, k_{2}^{\prime}, \ldots, k_{\ell}^{\prime}\right)$ with initial vertex $v$ such that $k_{i}^{\prime} \geq k_{i}$ if $i$ is odd, and $k_{i}^{\prime}=k_{i}$ otherwise.

Proof. This proof is by induction on $\ell$. If $\ell=1$, then the result holds trivially. Assume now $\ell \geq 2$, and suppose that, for every path $P\left(x_{1}, x_{2}, \ldots, x_{t}\right)$ with $t<\ell$ and every digraph $G$ with $\delta^{+}(G) \geq \sum_{i=1}^{t} x_{i}, G$ contains a path $P\left(x_{1}^{\prime}, x_{2}^{\prime}, \ldots, x_{t}^{\prime}\right)$ starting at any vertex of $G$ such that $x_{i}^{\prime} \geq x_{i}$ if $i$ is odd, and $x_{i}^{\prime}=x_{i}$ otherwise.

Let $v$ be a vertex of $D$. Since $\delta^{+}(D) \geq \sum_{i=1}^{\ell} k_{i}$ and $\ell \geq 2$, there exists a $(v, u)$-dipath $P_{v, u}$ in $D$ of length exactly $k_{1}$, for some vertex $u \in V(D)$. Let $D^{\prime}=D-\left(P_{v, u}-u\right)$, let $C$ be the connected component of $D^{\prime}$ containing $u$, and let $H$ be a sink strong component of $C$ (i.e. a strong component without arcs leaving it) that is reachable by a directed path in $C$ starting at $u$. We denote by $P_{u, x}$ a $(u, x)$-dipath in $C$ such that $V\left(P_{u, x}\right) \cap V(H)=\{x\}$.

Note that no vertex of $H$ dominates a vertex in $V\left(P_{u, x}\right) \backslash\{x\}$ since $H$ is a sink strong component. Thus, $\delta^{+}(H) \geq \delta^{+}(D)-k_{1} \geq k_{2}$. As a consequence, $H$ contains a directed cycle of length at least $k_{2}$. 
Using this and the fact that $H$ is strongly connected, we conclude that there exists a dipath $P_{y, x}$ in $H$ from a vertex $y \in V(H) \backslash\{x\}$ to $x$ of length exactly $k_{2}$. Let $G=H-\left(P_{y, x}-y\right)$. One may easily verify that $\delta^{+}(G)$ is at least $\delta^{+}(D)-k_{1}-k_{2} \geq \sum_{i=3}^{\ell} k_{i}$.

Let $Q_{v, y}=P_{v, u} P_{u, x} P_{y, x}$. Note that $Q_{v, y}$ is a path $P\left(k_{1}^{\prime}, k_{2}\right)$ starting at $v$ with $k_{1}^{\prime} \geq k_{1}$. Therefore, the result follows immediately if $\ell=2$. Suppose now that $\ell \geq 3$. By the induction hypothesis, $G$ contains a path $W_{y}:=P\left(k_{3}^{\prime}, \ldots, k_{\ell}^{\prime}\right)$ with initial vertex $y$ such that $k_{i}^{\prime} \geq k_{i}$ if $i$ is odd, and $k_{i}^{\prime}=k_{i}$ otherwise. Therefore, $Q_{v, y} W_{y}$ is the desired path $P\left(k_{1}^{\prime}, k_{2}^{\prime}, \ldots, k_{\ell}^{\prime}\right)$ with initial vertex $v$.

Since $\sum_{i=1}^{\ell} k_{i}=\left|V\left(P\left(k_{1}, k_{2}, \ldots, k_{\ell}\right)\right)\right|-1$, and that the complete digraph $\vec{K}_{k}$ on $k$ vertices has minimum out-degree $k-1$ and contains no path on more than $k$ vertices, we obtain the following corollary.

Corollary 14.2. mader $_{\delta^{+}}(P)=|V(P)|-1$ for every oriented path $P$.

\subsection{Subdivisions of in-arborescences}

The aim of this section is to show that, for every in-arborescence $T$, there exists an integer $f(T)$ such that every digraph with minimum out-degree at least $f(T)$ contains a subdivision of $T$. In other words, we shall prove that the in-arborescences are $\delta^{+}$-maderian.

To prove this, we need some preliminary results. The first one is the vertex and directed version of the celebrated Menger's theorem [58]. (See also [42] for a short proof.)

Theorem 14.3 (Menger's theorem). Let $D$ be a digraph, and let $S, T \subseteq V(D)$. The maximum number of vertex-disjoint $(S, T)$-dipaths is equal to the minimum size of an $(S, T)$-vertex-cut.

Lemma 14.4. Let $D$ be a digraph, let $S \subseteq V(D)$ be a nonempty subset of vertices of in-degree 0 in $D$, and let $T \subseteq V(D)$ such that $T \cap S=\emptyset$. If $d^{+}(v) \geq \Delta^{-}(D)$ for all $v \in V(D) \backslash T$, then there exist $|S|$ vertex-disjoint $(S, T)$-dipaths in $D$.

Proof. Suppose to the contrary that there do not exist $|S|$ vertex-disjoint $(S, T)$-dipaths in $D$. By Menger's theorem 14.3, there exists a set of vertices $X \subseteq V(D)$ with cardinality $|X|<|S|$ which is an $(S, T)$-vertexcut. Let $C$ be the set of vertices in $D-X$ that are reachable in $D$ by a dipath with initial vertex in $S \backslash X$. Set $k=|X \cap S|$. Observe that $k<|S|$.

Let us count the number $a(C, X)$ of arcs with tail in $C$ and head in $X$. Since the vertices in $S$ have in-degree 0 and every vertex in $C$ has out-degree at least $\Delta^{-}(D)$,

$$
a(C, X) \geq|C| \cdot \Delta^{-}(D)-[|C|-(|S|-k)] \cdot \Delta^{-}(D)=(|S|-k) \cdot \Delta^{-}(D) .
$$

Moreover, $a(C, X)$ is at most the number of arcs with head in $X$ which is at most $(|X|-k) \cdot \Delta^{-}(D)$, because the vertices in $S \cap X$ have in-degree 0. Hence $(|S|-k) \cdot \Delta^{-}(D) \leq a(C, X) \leq(|X|-k) \cdot \Delta^{-}(D)$. This is a contradiction to $|X|<|S|$.

Let $k$ and $\ell$ be positive integers. The $\ell$-branching in-arborescence of depth $k$, denoted by $B(k, \ell)$, is defined by induction as follows.

- $B(0, \ell)$ is the in-arborescence with a single vertex, which is the root and the leaf of $B(0, \ell)$.

- $B(k, \ell)$ is obtained from $B(k-1, \ell)$ by taking each leaf of $B(k-1, \ell)$ in turn and adding $\ell$ new vertices dominating this leaf. The root of $B(k, \ell)$ is the one of $B(k-1, \ell)$, and the leaves of $B(k, \ell)$ are the newly added vertices, that is, those not in $V(B(k-1, \ell))$. 
The in-arborescences $B(2,3)$ and $B(3,3)$ are depicted in Figure 14.2. The number of vertices of $B(k, \ell)$ is denoted by $b(k, \ell)$; so $b(k, \ell)=\sum_{i=0}^{k} \ell^{i}=\frac{1-\ell^{k+1}}{1-\ell}$.

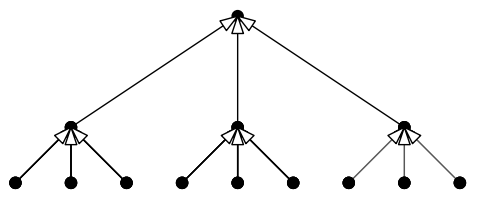

(a) In-arborescence $B(2,3)$.

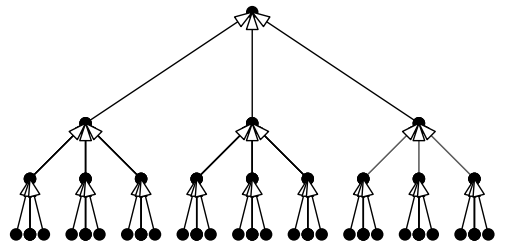

(b) In-arborescence $B(3,3)$.

Figure 14.2: Examples of in-arborescences.

Observe that every in-arborescence $T$ is a subdigraph of $B(k, \ell)$ with $k=\Delta^{-}(T)$ and $\ell$ the maximum length of a dipath in $T$. Therefore to prove that in-arboresences are $\delta^{+}$-maderian, it suffices to show that $B(k, \ell)$ is $\delta^{+}$-maderian for all $k$ and $\ell$.

Let us define the following recursive function $f: \mathbb{N} \rightarrow \mathbb{N}$. For all positive integers $k$ and $\ell$ such that $\ell \geq 2, f(1, \ell)=\ell$ and, for $k \geq 2$, we define

$$
f(k, \ell)=t(k, \ell) \cdot(\ell-1) \cdot k+t(k, \ell)
$$

where $t(k, \ell):=f(k-1, b(k-1, \ell) \cdot(\ell-1)+1) \cdot b(k-1, \ell)$.

If $\mathcal{D}$ is a family of digraphs, a packing of elements of $\mathcal{D}$ is the disjoint union of copies of elements of $\mathcal{D}$.

Theorem 14.5. Let $k$ and $\ell \geq 2$ be positive integers, and let $D$ be a digraph with $\delta^{+}(D) \geq f(k, \ell)$. Then $D$ contains a subdivision of $B(k, \ell)$.

Proof. We prove the result by induction on $k$ and $\ell$. If $k=1$, then $\delta^{+}(D) \geq \ell$. Thus, $\Delta^{-}(D) \cdot|V(D)| \geq$ $\sum_{v \in V(D)} d^{-}(v)=\sum_{v \in V(D)} d^{+}(v) \geq \ell \cdot|V(D)|$. Hence there is a vertex with in-degree at least $\ell$ in $D$ and, consequently, the result follows when $k=1$. Assume now $k \geq 2$, and suppose that, for every positive integers $k^{\prime}<k$ and $\ell^{\prime} \geq 2$, and every digraph $H$ with $\delta^{+}(H) \geq f\left(k^{\prime}, \ell^{\prime}\right), H$ contains a subdivision of the $\ell^{\prime}$-branching in-arborescence of depth $k^{\prime}$.

Let $\mathcal{F}$ be a packing of $\ell$-branching in-arborescences subdigraphs of any non-zero depth in $D$ that covers the maximum number of vertices. We denote by $U \subseteq V(D)$ the set of vertices not covered by $\mathcal{F}$, that is, $U=V(D) \backslash \bigcup_{A \in \mathcal{F}} V(A)$. Let $r_{A}$ denote the root of the in-arborescence $A$, for each $A \in \mathcal{F}$, and let $R=\left\{r_{A} \in V(D): A \in \mathcal{F}\right\}$ be the set of the roots of the arborescences in $\mathcal{F}$.

We now construct the digraph $H$ with vertex set $R$ such that there exists an $\operatorname{arc}\left(r_{A}, r_{B}\right)$ in $H$ if and only if $r_{A}$ dominates some vertex of $V(B)$ in $D$.

Claim 14.6. If $\delta^{+}(H) \geq t(k, \ell) / b(k-1, \ell)$, then $D$ contains a subdivision of $B(k, \ell)$.

Proof. Let $p=b(k-1, \ell) \cdot(\ell-1)+1$. By the induction hypothesis, $H$ contains a subdivision $T$ of $B(k-1, p)$. Let $R^{\prime}$ be the set of branching vertices of $T$, that is, $R^{\prime}=\left\{r \in V(T): d_{T}^{-}(r)=p\right\}$. We assume that each in-arborescence of $\mathcal{F}$ has at most $b(k-1, \ell)$ vertices, as any larger arborescence would yield the theorem. Thus, for each $r \in R^{\prime}$, there exists a vertex $h_{r}$ in the in-arborescence rooted at $r$ such that $h_{r}$ is dominated in $D$ by $\ell$ vertices of $V(T)$. Similarly, for each $r \in V(T)$ with in-degree 1 , there exists a vertex $h_{r}$ in the in-arborescence rooted at $r$ such that $h_{r}$ is dominated in $D$ by a vertex of $V(T)$. Using these remarks, we next define a procedure to obtain a subdigraph of $T$ that is a subdivision of $B(k-1, \ell)$. 
For each $r \in R^{\prime}$, we remove from $T$ all arcs with head $r$ but exactly $\ell$ arcs from vertices in $V(T)$ that dominate $h_{r}$ in $D$. We denote by $T^{\prime}$ the component of the subdigraph of $T$ obtained by applying the abovedescribed procedure and that contains the root of $T$. One may easily verify that $T^{\prime}$ is a subdivision of $B(k-1, \ell)$. Let $P_{r}$ be the path from $h_{r}$ to $r$ in the in-arborescence corresponding to $r$, for every $r \in V\left(T^{\prime}\right)$ such that either $r \in R^{\prime}$ or $r$ has in-degree 1 .

Let $Q$ be the in-arborescence obtained from $T^{\prime}$ in the following way. For each $r \in V\left(T^{\prime}\right)$ such that either $r \in R^{\prime}$ or $r$ has in-degree 1, we add $h_{r}$ to $T^{\prime}$, and we add an arc from every in-neighbour of $r$ in $T^{\prime}$ to $h_{r}$. Additionally, we remove all arcs with head $r$ in $T^{\prime}$, and link $h_{r}$ to $r$ by using the dipath $P_{r}$. Finally, for each $r \in V\left(T^{\prime}\right)$ that is a leaf, we replace $r$ by its corresponding in-arborescence belonging to $\mathcal{F}$.

By this construction, we have that $Q$ is a subdigraph of $D$ such that every internal vertex has either in-degree $\ell$ or 1 . Furthermore, it has depth at least $k$. Therefore, by possibly pruning some levels of $Q$, we obtain a subdivision of $B(k, \ell)$.

Suppose now $\delta^{+}(H)<t(k, \ell) / b(k-1, \ell)$. Observe that, for every $v \in R$ such that $d_{H}^{+}(v)<t(k, \ell) / b(k-$ $1, \ell)$, we have, in the digraph $D$, that $d_{U}^{+}(v) \geq t(k, \ell) \cdot(\ell-1) \cdot k$ since $\delta^{+}(D) \geq t(k, \ell) \cdot(\ell-1) \cdot k+t(k, \ell)$. We define

$$
X=\left\{v \in R: d_{U}^{+}(v) \geq t(k, \ell) \cdot(\ell-1) \cdot k\right\} .
$$

Let $D^{\prime}$ be the digraph obtained from $D[U \cup X]$ by removing all arcs with head in $X$. From $D^{\prime}$, we construct a digraph $G$ by replacing every vertex $v \in X$ by $t(k, \ell)$ new vertices $v_{1}, \ldots, v_{t(k, \ell)}$, and adding, for each $i \in[t(k, \ell)]$, at least $(\ell-1) \cdot k$ arcs from $v_{i}$ to $N_{D^{\prime}}^{+}(v)$ in such a way that $d_{D^{\prime}}^{-}(u)=d_{G}^{-}(u)$, for all $u \in N_{D^{\prime}}^{+}(v)$. In other words, we "redistribute" the out-neighbours of $v$ in $D^{\prime}$ among its $t(k, \ell) \operatorname{copies}$ in $G$ so that every copy has out-degree at least $(\ell-1) \cdot k$, and the in-degrees of vertices belonging to $U$ are not changed. Let $S \subseteq V(G)$ be the set of vertices that replaced those of $X$, that is, $S=\bigcup_{v \in X}\left\{v_{1}, \ldots, v_{t(k, \ell)}\right\}$. Let $T$ be the set of vertices in $U$ that have large out-degree outside $U$ in the digraph $D$, more formally, $T=\left\{v \in U: d_{V(D) \backslash U}^{+}(v) \geq t(k, \ell)+1\right\}$.

For every $i \in[k-1]$, let $\mathcal{F}_{i}=\{A \in \mathcal{F}: A$ has depth exactly $i\}$. Note that $\left\{\mathcal{F}_{i}\right\}_{i \in[k-1]}$ forms a partition of the packing $\mathcal{F}$. Additionally, observe that, due to the maximality of $\mathcal{F}$, every vertex in $U$ is dominated by at most $\ell-1$ vertices belonging to $U$, and by at most $\ell-1$ roots of in-arborescences in $\mathcal{F}_{i}$, for each $i \in[k-1]$. Thus, the in-degree in $G$ of every vertex belonging to $U$ is at most $(\ell-1)+(\ell-1) \cdot(k-1)=(\ell-1) \cdot k$. Therefore, we have $\Delta^{-}(G) \leq(\ell-1) \cdot k$. Moreover, since $\delta^{+}(D) \geq t(k, \ell) \cdot(\ell-1) \cdot k+t(k, \ell)$, we have $d_{G}^{+}(v) \geq t(k, \ell) \cdot(\ell-1) \cdot k$ for every $v \in U \backslash T$. Hence, $d_{G}^{+}(v) \geq(\ell-1) \cdot k$, for every $v \in V(G) \backslash T$. By Lemma 14.4, there exists a set $\mathcal{P}$ of $|S|$ vertex-disjoint paths from $S$ to $T$ in $G$.

Note that, in $D$, every vertex belonging to $T$ has at least $t(k, \ell)+1$ out-neighbours in $V(D) \backslash U$. Therefore one can greedily extend each path of $\mathcal{P}$ with an out-neighbour of its terminal vertex in $V(D) \backslash U$ in order to obtain a set $\mathcal{P}^{\prime}$ of $|S|$ vertex-disjoint paths from $S$ to $V(D) \backslash U$ such that for any $v \in X$ all the paths in $\mathcal{P}^{\prime}$ with initial vertex $v$ have distinct terminal vertices (and different from $v$ ).

We now construct the digraph $M$ on the vertex set $R$ where there exists an arc from $v=r_{A}$ to $r_{B}$ in $M$ whenever

either $r_{A}$ dominates some vertex of $V(B)$ in $D$,

or there is a dipath from some $v_{i}$ to $V(B)$ in $\mathcal{P}^{\prime}$.

Since, for each $v \in X$, all vertices in $\left\{v_{i}\right\}_{i \in[t(k, \ell)]}$ are the initial vertices of vertex-disjoint dipaths in $\mathcal{P}^{\prime}$, we obtain $\delta^{+}(M) \geq t(k, \ell) / b(k-1, \ell)$. Therefore, the result follows by Claim 14.6 with $M$ playing the role of $H$. 


\section{Part VI}

Conclusion 



\section{Chapter 15}

\section{Concluding remarks and open questions}

In this final chapter, we recall our main contributions for each of the problems addressed in this thesis. Furthermore, we present open questions which naturally lead to possible directions for future research.

\subsection{Convex recoloring}

In Chapter 4, we presented several results on the hardness of finding solutions for the convex recoloring problem. In particular, we showed that, for every $r$ and $k \geq 2$, the $r$-CR-BIN problem on $k$-colored $n$ vertex bipartite graphs cannot be approximated within a factor of $n^{1-\varepsilon}$ for any $\varepsilon>0$, unless $\mathcal{P}=\mathcal{N} \mathcal{P}$. This result shows that it is unlikely that there exists an approximation algorithm with ratio smaller than the ratio of a trivial algorithm that just assigns the same color to each vertex. Moreover, it improves the $\mathcal{O}(\log n)$ approximation threshold obtained by Campêlo et al. [21]. We also proved that the standard parameterization of $r$-CR-BIN is $\mathcal{W}[2]$-hard on $k$-colored bipartite graphs, for every $r$ and $k \geq 2$. Therefore, it is unlikely that there exists an FPT algorithm for this problem. This also strengthens the corresponding result in [21].

We proposed an integer linear programming formulation for the convex recoloring problem in Chapter 5. Our formulation, called (FMW), has an exponential number of variables and it is embedded in a column generation procedure in which a new column is efficiently generated when the input graph is a tree.

We provided computational experiments carried out with instances that come from an application in the study of phylogenetic trees (the same type of instances used in [19, 26]). We compared the proposed formulation with the one introduced by Chopra et al. in [26] which we called (CRLS). The computational experiments indicated that, on instances with low number of colors in the initial coloring, (CRLS) was faster than (FMW), whereas (FMW) performed better than (CRLS) when the number of colors is high. As we mentioned, this may be explained by the fact that the number of added variables in formulation (FMW) was very small (less than $|V(G)|$ in all cases), while the number of variables in formulation $(\mathrm{CRLS})$ is $\Theta((|V(G)|+|E(G)|) \cdot|\mathrm{C}|)$.

While writing the final version of this thesis, we came across the working paper Chopra et al. [25] which also proposes a column generation approach to the convex recoloring problem restricted to trees and presents computational experiments.

To inspire future works, we raise the following questions.

Question 15.1. Is there an efficient algorithm to solve the pricing problem for larger classes of graphs? 
Are formulations (FMW) and (CRLS) equivalent or does (FMW) dominate (CRLS)?

Question 15.2. What properties of the instances make them difficult to be solved by our column generation algorithm?

\subsection{On $k$-fold coloring}

In this thesis, we addressed the $k$-fold coloring problem on a graph $G$ from a polyhedral point of view. Although it is equivalent to the classic (1-fold) coloring problem on the graph $G \circ K_{k}$, the lexicographic product of $G$ by the clique on $k$ vertices, this transformation increases the size of the input and embeds many symmetries, which justifies an approach of the problem directly on $G$ [57]. We worked in this direction.

We proposed an ILP formulation for the problem based on class representatives and presented some basic properties of the polytope $\mathcal{P}_{k}(G)$ associated with this formulation. These are the subjects of Chapter 7 .

In Chapters 8 and 9, we studied the facial structure of $\mathcal{P}_{k}(G)$.

First, we profited from the known results for $\mathcal{P}_{1}(G)$ described in [16] to study the facial structure of $\mathcal{P}_{k}(G)$. In Section 8.1, we presented a lifting theorem that consider nontrivial combinations of facetdefining inequalities of $\mathcal{P}_{k}(G)$ and $\mathcal{P}_{\ell}(G)$ in order to get a facet-defining inequality of $\mathcal{P}_{k+\ell}(G)$. Additionally, we showed specific liftings from facet-defining inequalities of $\mathcal{P}_{k}(G)$ to facet-defining inequalities of $\mathcal{P}_{k+1}(G)$. In Section 8.2, we dealt with the representatives coloring polytope of $G \circ K_{k}$. We showed how to project facet-defining inequalities from $\mathcal{P}_{1}\left(G \circ K_{k}\right)$ into facet-defining inequalities of $\mathcal{P}_{k}(G)$. We also studied the relationship between the representatives $k$-fold coloring polytope and the stable set polytope. In Section 8.3, we showed how to transform to $\mathcal{P}_{k}(G)$ any facet-defining inequality of the stable set polytope associated with the subgraph of $G$ induced by the anti-neighborhood of each vertex. We remark that relations between the coloring polytope and the stable set polytope have been considered before. In [30], it is shown a correspondence between the (1-fold) coloring polytope associated with $\mathcal{R}_{1}(G)$ with equality in (7.2) and the stable set polytope of a subgraph of the line graph of $G$. A similar correspondence is used in [64] to derive facet-defining inequalities for this coloring polytope. In this work, we used a different strategy. We showed a transformation from facets of the stable set polytope of subgraphs of $G$ itself. Moreover, we do not restrict ourselves to $k=1$.

Finally, in Sections 9.2 and 9.3, we derived a class of facet-defining inequalities associated with induced subgraphs, namely webs and antiwebs. These classes of graphs play an important role in the description of stable set and coloring polytopes $[24,78]$. We also characterized a criticality property required by these graphs to induce facets of $\mathcal{P}_{k}(G)$.

We were able to recover to $\mathcal{P}_{k}(G)$ most of the facet-defining inequalities of the coloring polytope derived in [16, 64], either by simply keeping their coefficients or by lifting them. In addition, the results on webs and antiwebs extend and generalize the results in [16, 65].

As a future research direction, it would be interesting to carry out a computational comparison between the representatives formulation and other formulations described in the literature (e.g. the maximal independent sets formulation [57]). Of course, generating good cuts for our representatives formulation is a key point for its good performance. Thus, it would be necessary to study the separation problems associated with the facet-defining inequalities induced by webs and antiwebs aiming the development of (efficient) algorithms for these problems. 


\subsection{Proper orientation}

In our work on the proper orientation problem, we showed upper bounds for the proper orientation number on some classes of bipartite graphs and we proved several hardness results. More precisely, in Chapter 11, we showed that

$$
\text { if } G \text { is bipartite, then } \vec{\chi}(G) \leq\left\lfloor\frac{\Delta(G)+\sqrt{\Delta(G)}}{2}\right\rfloor+1 .
$$

In the case of trees, we showed that $\vec{\chi}(T) \leq 4$ for every tree $T$. We remark that a simpler proof to this result was recently given by Knox et al. [51]. Their proof leads to a polynomial-time algorithm to proper orienting trees within this bound.

The computational complexity of computing the proper orientation number was studied in Chapter 12. Ahadi and Dehghan [3] proved that it is $\mathcal{N} \mathcal{P}$-complete to decide whether $\vec{\chi}(G)=2$ for planar graphs $G$. We first improve this result by showing that it is $\mathcal{N} \mathcal{P}$-complete to decide whether $\vec{\chi}(G) \leq 2$ for planar subcubic graphs $G$. Additionally, we proved that, for every $k \geq 3$, it is $\mathcal{N} \mathcal{P}$-complete to decide whether $\vec{\chi}(G) \leq \Delta(G)-1$ for graphs $G$ with $\Delta(G)=k$. Finally, we showed that it is also $\mathcal{N} \mathcal{P}$-complete to decide whether $\vec{\chi}(G) \leq 3$ for planar bipartite graphs $G$ with maximum degree 5 .

A split graph is a graph whose vertex set may be partitioned into a clique $K$ and a stable set $S$. We assume, without loss of generality, that $K$ is maximal, that is no vertex in $S$ is adjacent to all vertices in $K$. The pair $(K, S)$ is then called a canonical partition of $G$. For such a partition, we have $\omega(G)=|K|$. It is well-known that split graphs can be recognized in polynomial time, and that finding a canonical partition of a split graph can also be done in polynomial time.

Proposition 15.3. If $G$ is a split graph, then one can decide in polynomial time whether $\vec{\chi}(G)=$ $\omega(G)-1$.

Proof. Let $(K, S)$ be a canonical partition of $G$. Let $H$ be the following bipartite graph obtained from $G$ : the vertex set of $H$ is $K \cup\left\{w_{0}, \ldots, w_{|K|-1}\right\}$ and, for every $v \in K$ and $i \in\{0, \ldots,|K|-1\}$, there exists an edge between $v$ and $w_{i}$ if and only if $v$ has no neighbor (in $S$ ) with degree $i$ in $G$.

In what follows, we prove that $\vec{\chi}(G)=\omega(G)-1$ if and only if $H$ has a perfect matching.

Suppose that there exists a proper orientation $D$ of $G$ with maximum in-degree $|K|-1$. By Lemma 12.2, all edges between $K$ and $S$ must be oriented from $K$ to $S$, so $d_{D}^{-}(u)=d_{G}(u)$ for all $u \in S$. It follows that, for each $v \in K, d_{D}^{-}(v) \notin\left\{d_{G}(u): u \in S \cap N(v)\right\}$. Moreover, there exists exactly one vertex in $K$ of in-degree $i$, for each $i \in\{0, \ldots,|K|-1\}$. Therefore, the edge set $\left\{v w_{i}: v \in K\right.$ and $\left.i=d_{D}^{-}(v)\right\}$ is a perfect matching of $H$.

Suppose now that $H$ has a perfect matching $M$. Consider the orientation of $G$ defined as follows. We orient the edges in the clique $K$ so that $v \in K$ has in-degree $i$ if and only if $v w_{i} \in M$, where $i \in$ $\{0, \ldots,|K|-1\}$. Additionally, we orient all edges between $K$ and $S$ towards $S$ in the graph $G$. Note that $d_{G}(u)<|K|-1$ for all $u \in S$ since $(K, S)$ is a canonical partition. By the construction of $H$, the orientation described above is a proper $(\omega(G)-1)$-orientation of $G$.

However, one further step does not seem to be trivial.

Question 15.4. Can one decide in polynomial time whether $\vec{\chi}(G) \leq \omega(G)$ for every split graph $G$ ?

Similarly, one may ask the following question. 
Question 15.5. Can one decide in polynomial time whether $\vec{\chi}(G)=\omega(G)-1$ for every complement of bipartite graph $G$ ?

Another interesting direction for further research is in terms of bounds for the proper orientation number. Recall that we proved that the proper orientation number of every tree is at most 4 . Very recently, Knox et al. [51] proved that 3-connected planar bipartite graphs have proper orientation number at most 6. Moreover, Araujo et al. [7] showed that every cactus admits a proper orientation with maximum indegree at most 7 . Therefore, a natural direction for future is research follows from the following question.

Question 15.6. Does there exist a constant $k$ such that $\vec{\chi}(G) \leq k$ for all planar graphs $G$ ?

\subsection{Subdivisions of digraphs}

In Chapter 14, we gave new evidences for Mader's conjecture on subdivisions of digraphs. Firstly, in Section 14.1, we proved that every oriented path $P$ (that is, orientation of an undirected path) is $\delta^{+}$-maderian and that $\operatorname{mader}_{\delta^{+}}(P)=|V(P)|-1$. In Section 14.2, we tackled the case of arborescences. Recall that an out-arborescence (respectively, in-arborescence) is an oriented tree in which all arcs are directed away from (respectively, towards) a vertex called root. Trivially, the simple greedy procedure shows that $\operatorname{mader}_{\delta^{+}}(T)=|T|-1$ for every out-arborescence $T$. In contrast, the fact that in-arborecences are $\delta^{+}$-maderian is not obvious since we have no control on the in-degree of every vertex in a digraph of large minimum out-degree. We proved that the in-arborescences are $\delta^{+}$-maderian.

Based on our contributions, a natural next step towards a proof of Mader's conjecture is to answer the following question.

Question 15.7. Is every oriented tree $\delta^{+}$-maderian?

One may also consider graph parameters different from the minimum out-degree. Note that every graph with chromatic number at least $p$ has a subgraph with minimum degree at least $p-1$. This implies, by Theorem 13.1, that every graph with chromatic number at least $g(k)+1$ contains a subdivision of $K_{k}$. In the context of digraphs, there exist two natural analogues of the chromatic number. Given a digraph $D$, the chromatic number of $D$, denoted by $\chi(D)$, is simply the chromatic number of its underlying graph. The dichromatic number of $D$, denoted by $\vec{\chi}(D)$, is the smallest integer $k$ such that $D$ admits a $k$-dicoloring. A $k$-dicoloring is a $k$-partition $\left\{V_{1}, \ldots, V_{k}\right\}$ of $V(D)$ such that $D\left[V_{i}\right]$ is acyclic for every $i \in[k]$. Hence, it is natural to ask which digraphs are $\chi$-maderian and which ones are $\vec{\chi}$-maderian.

Burr [15] proved that every $(k-1)^{2}$-chromatic digraph contains every oriented forest of order $k$. Later on, Addario-Berry et al. [2] slightly improved this value to $k^{2} / 2-k / 2+1$. This implies that every oriented forest is $\chi$-maderian. Cohen et al. [29] showed that for any positive integer $b$, there are digraphs of arbitrarily high chromatic number that contain no oriented cycles with less than $b$ blocks. This directly implies that if a digraph is not an oriented forest, then it is not $\chi$-maderian because it contains an oriented cycle, all subdivisions of which have the same number of blocks.

Theorem 15.8 (Cohen et al. [29]). A digraph is $\chi$-maderian if and only if it is an oriented forest.

The $\chi$-maderian digraphs are known but determining mader $_{\chi}$ for such digraphs is still open. In [15], Burr conjectured the following.

Conjecture 15.9 (Burr [15]). Every digraph with chromatic number $2 k-2$ contains every oriented tree of order $k$ as a subdigraph. 
An interesting step towards Burr's conjecture is to answer the following question.

Question 15.10. Does mader $_{\chi}(T) \leq 2|V(T)|-2$ hold for every oriented tree $T$ ?

In [1], it was proven that every digraph is $\vec{\chi}$-maderian. Again determining mader $_{\vec{\chi}}$ for every digraph is still open. Since every digraph $D$ of order $n$ is a subdigraph of $\vec{K}_{n}$, the complete digraph of order $n$, and so $\operatorname{mader}_{\vec{\chi}}(D) \leq \operatorname{mader}_{\vec{\chi}}\left(\vec{K}_{n}\right)$, it is natural to focus on $\vec{K}_{n}$.

Question 15.11. What is $\operatorname{mader}_{\vec{\chi}}\left(\vec{K}_{n}\right)$ ?

Aboulker et al. [1] showed that $\operatorname{mader}_{\vec{\chi}}\left(\vec{K}_{n}\right) \leq 4^{n^{2}-2 n+1}(n-1)+1$ and, more generally, that if $F$ is a digraph with $n$ vertices, $m$ arcs and $c$ connected components, then $\operatorname{mader}_{\vec{\chi}}(F) \leq 4^{m-n+c}(n-1)+1$. 


\section{Bibliography}

[1] Aboulker, P., Cohen, N., Havet, F., Lochet, W., Moura, P. F. S., and Thomassé, S. Subdivisions in digraphs of large out-degree or large dichromatic number. ArXiv e-prints (Oct. 2016). 98, 109

[2] Addario-Berry, L., Havet, F., Sales, C. L., Reed, B., and Thomassé, S. Oriented trees in digraphs. Discrete Mathematics 313, 8 (2013), 967 - 974. 108

[3] Ahadi, A., and Dehghan, A. The complexity of the proper orientation number. Information Processing Letters 113, 19-21 (2013), 799-803. 71, 72, 73, 79, 85, 107

[4] Akhoondian Amiri, S., Kawarabayashi, K.-I., Kreutzer, S., and Wollan, P. The ErdősPosa Property for Directed Graphs. ArXiv e-prints (Mar. 2016). 97

[5] Alon, N. Disjoint directed cycles. Journal of Combinatorial Theory, Series B 68, 2 (1996), 167 178. 97

[6] Araujo, J., Cohen, N., de Rezende, S. F., Havet, F., and Moura, P. F. On the proper orientation number of bipartite graphs. Theoretical Computer Science 566, 0 (2015), 59-75. 73

[7] Araujo, J., Havet, F., Sales, C. L., And Silva, A. Proper orientation of cacti. Theoretical Computer Science 639 (2016), 14-25. 72, 108

[8] Bahiense, L., Frota, Y., Noronha, T. F., and Ribeiro, C. C. A branch-and-cut algorithm for the equitable coloring problem using a formulation by representatives. Discrete Appl. Math. 164 (Feb. 2014), 34-46. 40, 43

[9] Bang-Jensen, J., And Gutin, G. Z. Digraphs: Theory, Algorithms and Applications, 1st ed. Springer-Verlag London Berlin Heidelberg, 2000. 7

[10] Bar-Yehuda, R., Kutiel, G., And Rawitz, D. 1.5-approximation algorithm for the 2-convex recoloring problem. In Proceedings of the 26th International Workshop, IWOCA 2015, Verona, Italy, October 5-7, 2015, Revised Selected Papers (2016), Springer International Publishing, pp. 299-311. 16

[11] Berg, M. D., And Khosravi, A. Optimal binary space partitions for segments in the plane. International Journal of Computational Geometry \& Applications 22, 3 (2012), 187-205. 89

[12] Bermond, J. C., And Thomassen, C. Cycles in digraph - a survey. Journal of Graph Theory 5, 1 (1981), 1-43. 97

[13] Bollobás, B., And Thomason, A. Proof of a conjecture of Mader, Erdös and Hajnal on topological complete subgraphs. European Journal of Combinatorics 19, 8 (1998), 883 - 887. 96

[14] Bondy, J. A., And Murty, U. S. R. Graph Theory, 3 ed., vol. 244 of Graduate Texts in Mathematics. Springer-Verlag, 2008. 7

[15] Burr, S. A. Subtrees of directed graphs and hypergraphs. In Proceedings of the Eleventh Southeastern Conference on Combinatorics, Graph Theory and Computing (Florida Atlantic Univ., Boca Raton, Fla., 1980), Vol. I (1980), vol. 28, pp. 227-239. 108 
[16] Campêlo, M., Campos, V., And CorrêA, R. On the asymmetric representatives formulation for the vertex coloring problem. Discrete Applied Mathematics 156, 7 (2008), 1097-1111. 40, 41, 43, 44, $45,48,49,50,54,55,57,58,106$

[17] Campêlo, M., Corrêa, R., And Frota, Y. Cliques, holes and the vertex coloring polytope. Information Processing Letters 89, 4 (2004), 159-164. 40, 43

[18] Campêlo, M., Corrêa, R., Moura, P. F. S., and Santos, M. C. On optimal $k$-fold colorings of webs and antiwebs. Discrete Applied Mathematics 161, 1-2 (2013), 60-70. 39, 40, 45, 57, 58, 59, 62,64

[19] Campêlo, M., Freire, A. S., Lima, K. R., Moura, P. F. S., and Wakabayashi, Y. The convex recoloring problem: polyhedra, facets and computational experiments. Mathematical Programming 156, 1 (2016), 303-330. 17, 32, 34, 35, 105

[20] Campêlo, M., Moura, P. F., And Santos, M. C. Lifted, projected and subgraph-induced inequalities for the representatives $k$-fold coloring polytope. Discrete Optimization 21 (2016), 131156. 41

[21] Campêlo, M. B., Huiban, C. G., Sampaio, R. M., and Wakabayashi, Y. Hardness and inapproximability of convex recoloring problems. Theoretical Computer Science 533 (2014), 15-25. $16,17,19,105$

[22] Catanzaro, D., Godi, A., And Labbé, M. A class representative model for pure parsimony haplotyping. INFORMS J. on Computing 22, 2 (Apr. 2010), 195-209. 40, 43

[23] Cheng, E., And de VRIes, S. Antiweb-wheel inequalities and their separation problems over the stable set polytopes. Mathematical Programming 92 (2002), 153-175. 54, 57

[24] Cheng, E., And de VRIEs, S. On the facet-inducing antiweb-wheel inequalities for stable set polytopes. SIAM J. on Discrete Mathematics 15, 4 (2002), 470-487. 41, 54, 57, 106

[25] Chopra, S., Erdem, E., Kim, I., And Shim, S. Column generation approach to the convex recoloring problem on a tree. In Proceedings of Modeling and Optimization: Theory and Applications (MOPTA) (2016), pp. 1-12. to appear. 105

[26] Chopra, S., Filipecki, B., Lee, K., Ryu, M., Shim, S., and Van Vyve, M. An extended formulation of the convex recoloring problem on a tree. Mathematical Programming (2016), 1-20. $17,28,32,33,105$

[27] Chor, B., Fellows, M., Ragan, M., Razgon, I., Rosamond, F., and Snir, S. Connected coloring completion for general graphs: Algorithms and complexity. Lecture Notes in Computer Science (including subseries Lecture Notes in Artificial Intelligence and Lecture Notes in Bioinformatics) 4598 (2007), 75-85. 15, 16, 19

[28] Chvátal, V., Garey, M., and Johnson, D. Two results concerning multicoloring. In Algorithmic Aspects of Combinatorics, P. H. B. Alspach and D. Miller, Eds., vol. 2 of Annals of Discrete Mathematics. Elsevier, 1978, pp. 151-154. 40

[29] Cohen, N., Havet, F., Lochet, W., And Nisse, N. Subdivisions of oriented cycles in digraphs with large chromatic number. Research Report RR-8865, LRI - CNRS, University Paris-Sud ; LIP - ENS Lyon ; INRIA Sophia Antipolis - I3S, Feb. 2016. 108

[30] Cornaz, D., And Jost, V. A one-to-one correspondence between colorings and stable sets. Operations Research Letters 36, 6 (2008), 673-676. 106

[31] Cygan, M., Fomin, F., Kowalik, Ł., Lokshtanov, D., Marx, D., Pilipczuk, M., Pilipczuk, M., And Saurabh, S. Parameterized Algorithms, 1 ed., vol. 1. Springer International Publishing, 2015. 7,10

[32] Dantchev, S., Martin, B., and Szeider, S. Parameterized proof complexity. Computational Complexity 20, 1 (2011), 51-85. 23 
[33] de Carvalho, M. H., Cerioli, M. R., Dahab, R., Feofiloff, P., Fernandes, C. G., Ferreira, C. E., aes, K. S. G., Miyazawa, F. K., de Pina Jr., J. C., Soares, J. A. R., and WAKABAYASHI, Y. Uma introdução sucinta a algoritmos de aproximação, 1 ed. may 2001. 7

[34] Diestel, R. Graph Theory, 4 ed., vol. 173 of Graduate Texts in Mathematics. Springer-Verlag, 2010. 7

[35] Dirac, G. A. In abstrakten Graphen vorhandene vollständige 4-Graphen und ihre Unterteilungen. Mathematische Nachrichten 22, 1-2 (1960), 61-85. 95

[36] Downey, R., And Fellows, M. Parameterized complexity. Monographs in Computer Science. Springer-Verlag, 1999. 7, 10

[37] Eisenbrand, F., Oriolo, G., Stauffer, G., and Ventura, P. The stable set polytope of quasi-line graphs. Combinatorica 28 (2008), 45-67. 54, 57

[38] Ferreira, C. E., and Wakabayashi, Y. Combinatória Poliédrica e Planos-de-Corte Faciais, 1 ed. X Escola de Computação-UNICAMP, 1996. 7

[39] Flum, J., And Grohe, M. Parameterized Complexity Theory (Texts in Theoretical Computer Science. An EATCS Series), 1 ed., vol. 1. Springer-Verlag New York, Inc., Secaucus, NJ, USA, 2006. 7

[40] Galluccio, A., and Sassano, A. The rank facets of the stable set polytope for claw-free graphs. Journal of Combinatorial Theory, Series B 69, 1 (1997), 1-38. 54, 57

[41] Giles, R., and Trotter, L. E. On stable set polyhedra for $K_{1,3}$-free graphs. Journal of Combinatorial Theory, Series B 31, 3 (1981), 313-326. 54, 57

[42] GöRING, F. Short proof of Menger's theorem. Discrete Mathematics 219, 1-3 (2000), 295-296. 100

[43] Hochbaum, D., And Pathria, A. Node-optimal connected $k$-subgraphs. Manuscript, April 1994. 31

[44] Jans, R., ANd Desrosiers, J. Binary clustering problems: symmetric, asymmetric and decomposition formulations. Tech. Rep. G-2010-44, Les Cahier du GERAD, 2010. 40, 43

[45] Jans, R., And Desrosiers, J. Efficient symmetry breaking formulations for the job grouping problem. Computers \& 3 Operations Research 40, 4 (2013), 1132 - 1142. 40, 43

[46] Johansson, A. Asymptotic choice number for triangle free graphs. Technical Report 91-95, DIMACS, 1996. 71

[47] Kammer, F., and Tholey, T. The complexity of minimum convex coloring. Discrete Applied Mathematics 160 (2012), 810-833. 16

[48] Kanj, I. A., And Kratsch, D. Convex recoloring revisited: Complexity and exact algorithms. In Proceedings of the 15th Annual International Conference on Computing and Combinatorics (2009), COCOON '09, pp. 388-397. 16

[49] Kawarabayashi, K.-I., and Kreutzer, S. The directed grid theorem. In Proceedings of the Forty-Seventh Annual ACM on Symposium on Theory of Computing (New York, NY, USA, 2015), STOC '15, ACM, pp. 655-664. 97

[50] Klostermeyer, W., and Zhang, C. Q. n-tuple coloring of planar graphs with large odd girth. Graphs and Combinatorics 18, 1 (2002), 119-132. 40

[51] Knox, F., González Hermosillo de la Maza, S., Mohar, B., and linhares Sales, C. Proper Orientations of Planar Bipartite Graphs. ArXiv e-prints (Sept. 2016). 72, 107, 108

[52] Komlós, J., And Szemerédi, E. Topological cliques in graphs II. Combinatorics, Probability and Computing 5 (3 1996), 79-90. 96 
[53] Lichtenstein, D. Planar formulae and their uses. SIAM Journal on Computing 11, 2 (1982), 329-343. 85

[54] Lima, K. R., and Wakabayashi, Y. Convex recoloring of paths. Discrete Appl. Math. 164, part 2 (2014), 450-459. 16

[55] MAder, W. Homomorphieeigenschaften und mittlere Kantendichte von Graphen. Mathematische Annalen 174 (1967), 265-268. 95

[56] Mader, W. Degree and local connectivity in digraphs. Combinatorica 5, 2 (1985), 161-165. 4, 5, 96

[57] Menrotra, A., And Trick, M. A branch-and-price approach for graph multi-coloring. In Extending the Horizons: Advances in Computing, Optimization, and Decision Technologies, E. K. Baker, A. Joseph, A. Mehrotra, and M. A. Trick, Eds., vol. 37. 2007, pp. 15-29. 106

[58] Menger, K. Zur allgemeinen Kurventheorie. Fundamenta Mathematicae 10, 1 (1927), 96-115. 100

[59] Moran, S., AND SNIR, S. Convex recolorings of strings and trees: Definitions, hardness results, and algorithms. In Proceedings WADS 2005: 9th International Workshop on Algorithms and Data Structures (2005), pp. 218-232. 16

[60] Moran, S., And Snir, S. Efficient approximation of convex recolorings. Journal of Computer and System Sciences 73, 7 (2007), 1078-1089. 16, 33

[61] Moran, S., And Snir, S. Convex recolorings of strings and trees: Definitions, hardness results and algorithms. Journal of Computer and System Sciences 74, 5 (2008), 850-869. 16, 33

[62] Nemhauser, G. L., And Wolsey, L. A. Integer and Combinatorial Optimization. WileyInterscience Series in Discrete Mathematics and Optimization. Wiley-Interscience, 1999. 7

[63] Oriolo, G., and Stauffer, G. Clique-circulants and the stable set polytope of fuzzy circular interval graphs. Mathematical Programming 115 (2008), 291-317. 54, 57

[64] Palubeckis, G. On the graph coloring polytope. Information Technology And Control 37, 1 (2008), 7-11. 106

[65] Palubeckis, G. Facet-inducing web and antiweb inequalities for the graph coloring polytope. Discrete Applied Mathematics 158 (2010), 2075-2080. 41, 57, 58, 106

[66] PÊCher, A., ANd Wagler, A. K. Almost all webs are not rank-perfect. Mathematical Programming 105 (2006), 311-328. 57

[67] Reed, B. $\omega, \delta$, and $\chi$. Journal of Graph Theory 27, 4 (1998), 177-212. 71

[68] Reed, B., Robertson, N., Seymour, P., and Thomas, R. Packing directed circuits. Combinatorica 16, 4 (1996), 535-554. 97

[69] Ren, G., AND Bu, Y. k-Fold coloring of planar graphs. Science China Mathematics 53, 10 (October 2010), 2791-2800. 40

[70] Scheinerman, E. R., And Ullman, D. H. Fractional Graph Theory: A Rational Approach to the Theory of Graphs. Wiley-Interscience, 1997. 40

[71] Sonres, J. C. O problema da atribuição conexa. Master's thesis, Federal University of Ceará, Brazil, 2016. 28

[72] Stahl, S. n-Tuple colorings and associated graphs. Journal of Combinatorial Theory 20 (1976), 185-203. 39, 40

[73] Stahl, S. The multichromatic numbers of some Kneser graphs. Discrete Mathematics 185, 1-3 (1998), 287-291. 40 
[74] Thomassen, C. Some homeomorphism properties of graphs. Mathematische Nachrichten 64, 1 (1974), 119-133. 96

[75] Thomassen, C. $K_{5}$-subdivisions in graphs. Combinatorics, Probability and Computing 5 (6 1996), 179-189. 96

[76] Trotter, L. E. A Class of facet producing graphs for vertex packing polyhedra. Discrete Mathematics 12 (1975), 373-388. 54, 57

[77] Vazirani, V. V. Approximation algorithms. Springer-Verlag New York, Inc., New York, NY, USA, 2001. 7

[78] Wagler, A. K. Antiwebs are rank-perfect. 4OR: A Quarterly Journal of Operations Research 2 (2004), 149-152. 41, 57, 106

[79] Xavier, Á. S., And CAmpêlo, M. A new facet generating procedure for the stable set polytope. Electronic Notes in Discrete Mathematics 37 (2011), 183-188. 50

[80] ZuCKerman, D. Linear degree extractors and the inapproximability of max clique and chromatic number. Theory of Computing 3, 6 (2007), 103-128. 39 